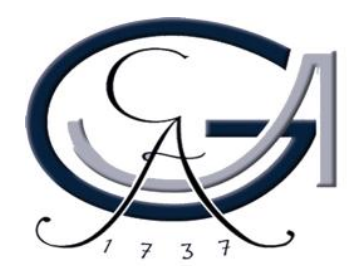

GEORG-AUGUST-UNIVERSITÄT GÖTTINGEN

\title{
Analysis of CRM1- and Nup214- dependent nuclear export of proteins
}

Dissertation for the award of the degree

"Doctor rerum naturalium"

Division of Mathematics and Natural Sciences

of the Georg-August-University Göttingen

submitted by

Stephanie Roloff

from Prenzlau

Göttingen 2012 
Member of the Thesis Committee (First Reviewer):

Prof. Dr. Ralph H. Kehlenbach

Department of Biochemistry I

Center for Biochemistry and Molecular Cell Biology

Georg-August University, Goettingen

Member of the Thesis Committee (Second Reviewer):

\section{Prof. Dr. Peter Rehling}

Department of Biochemistry II

Center for Biochemistry and Molecular Cell Biology

Georg-August University, Goettingen

Member of the Thesis Committee:

Prof. Dr. Ralf Ficner

Dept. of Molecular Structural Biology

Institute for Microbiology and Genetics

Georg-August University, Goettingen 
Statutory declaration:

I declare that this thesis has been written entirely by myself with no other sources and aids than explicitly quoted.

This thesis was not submitted in the same or in a substantially similar version to any other authority to achieve an academic grading and was not published elsewhere.

Stephanie Roloff 
To Science 


\begin{abstract}
Nuclear pore complexes (NPCs) are giant units of the nuclear envelope, mediating directional transport into and out of the nucleus. Nucleoporins are the main components of the NPC, and one third of them contain phenylalanine-glycine (FG) repeats. They are believed to serve as binding sites for transport receptors of the karyopherin family. CRM1 is the main export receptor in eukaryotic cells. It has a ring-like structure that is composed of HEAT (Huntingtin Elongation Factor A Subunit TOR) repeats (HRs) and exports various cargo proteins containing a leucine-rich nuclear export signal (NES). In the nucleus, the export complex assembles together with the small GTPase Ran in its GTPbound state. On its way through the nuclear pore, the trimeric export complex interacts with several nucleoporins, among them Nup214. Nup214 is located at the cytoplasmic side of the NPC and its FGrich C-terminus interacts with CRM1 in a RanGTP-dependent manner, providing a terminal docking site for CRM1-RanGTP-cargo complexes in the late steps of nuclear export. This interaction also indicates a specific role for Nup214 in nuclear export, at least for some cargos. Whereas the CRM1binding region in Nup214 is known, the CRM1-binding sites for Nup214 have not been identified so far. In this work, we analyzed the localization and the export potential of CRM1-fragments, mutants and chimeras, derived from H. sapiens and C. elegans CRM1. Whereas CRM1-fragments did not associate with the nuclear envelope and were not able to mediate export in mammalian cells, we found one CRM1-chimera which was located at the nuclear envelope and was able to transport NES-cargos through the nuclear pore. From that we conclude that association of CRM1 with the nuclear pore correlates with the ability to promote nuclear export. By comparing several chimeras with respect to their localization and functionality, we hypothesize that Nup214 binding occurs between HRs 9-11 of CRM1.

Transport receptors bind preferentially to FGs in nucleoporins. For CRM1 this had not been analyzed in detail. Here we show that CRM1 specifically binds to the FGs in Nup214. Moreover, we could narrow down the Nup214 region for CRM1-binding. By analyzing short Nup214-fragments and mutants, we identified a prominent FG-motif in Nup214, comprising 20 amino acid residues. This motif interacts with CRM1 in a RanGTP-dependent manner, with enhanced binding in the presence of an NES-cargo. Two other Nup214 regions downstream of this motif contribute to the stability of this interaction.
\end{abstract}




\section{Table of contents}

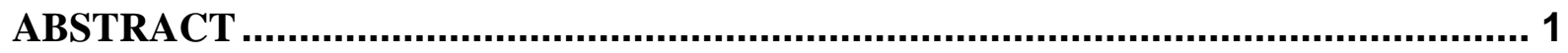

1 INTRODUCTION ........................................................................................ 5

$1.1 \quad$ The nuclear pore complex.........................................................................................................................5

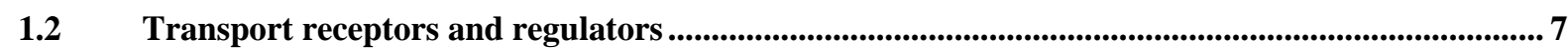

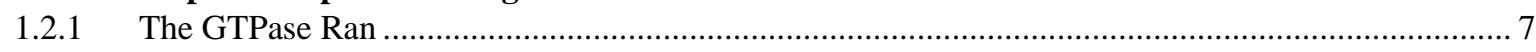

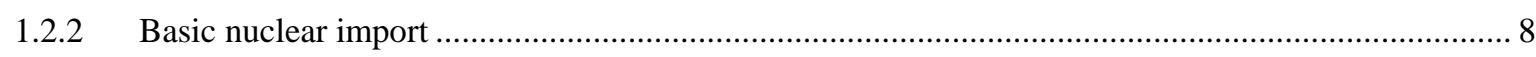

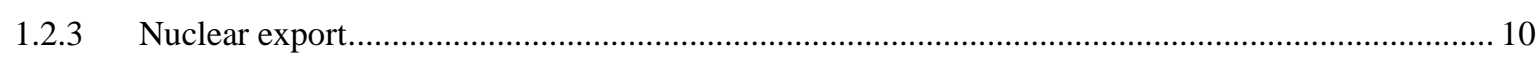

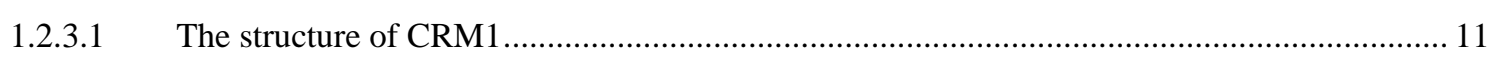

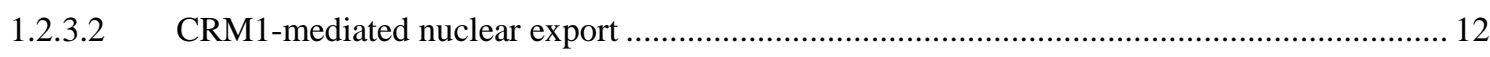

1.3 The translocation of transport complexes through the nuclear pore .................................................. 14

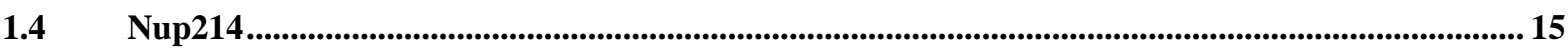

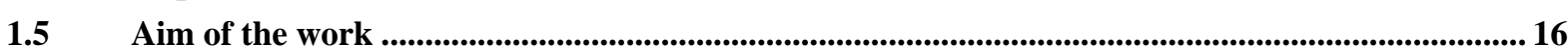

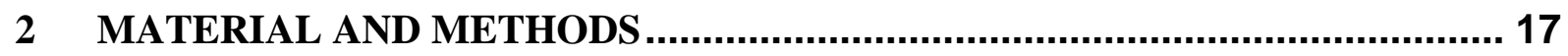

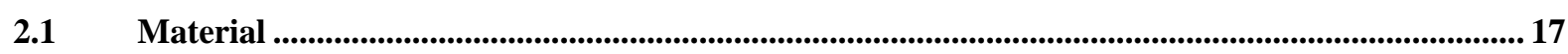

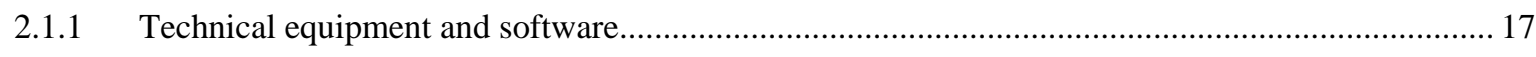

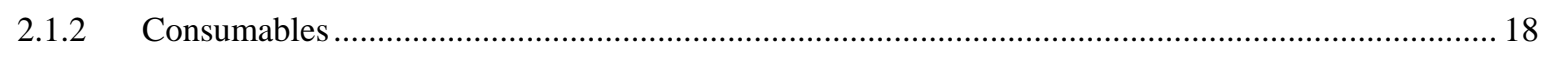

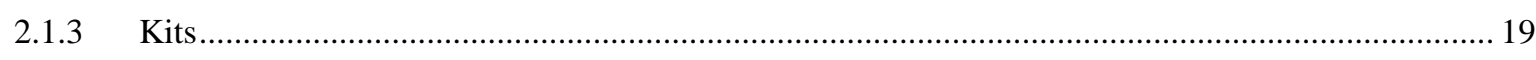

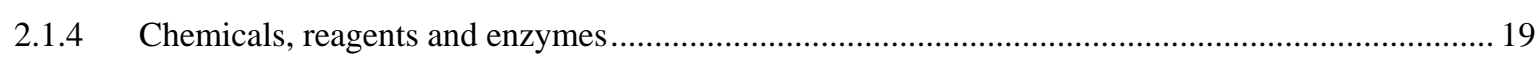

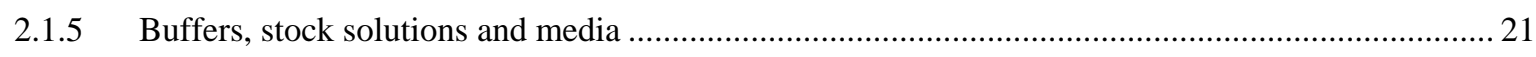

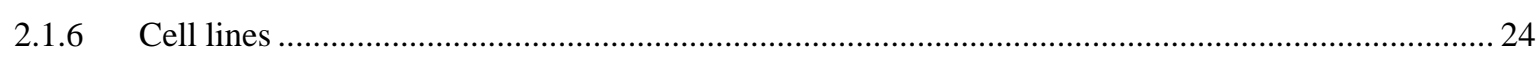

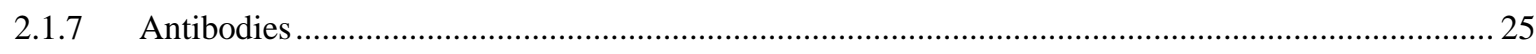

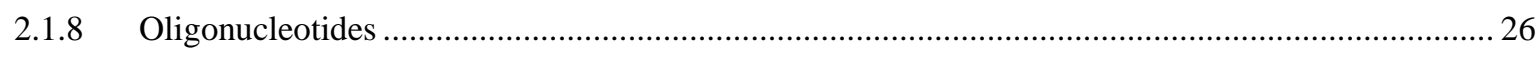

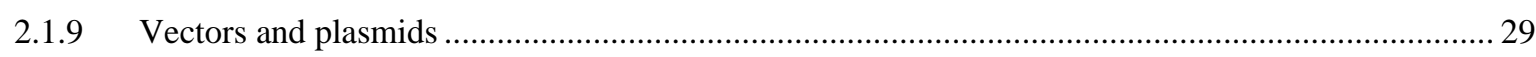

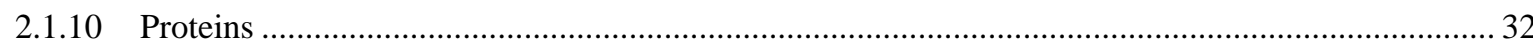

2.2 Biochemical methods ......................................................................................................................... 33

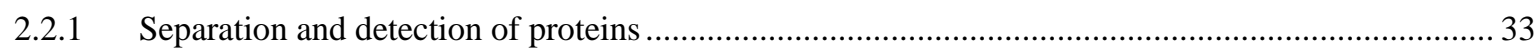

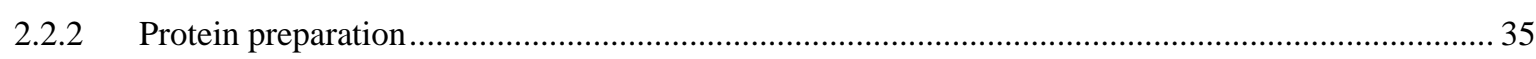

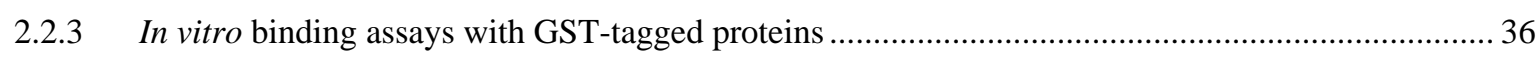

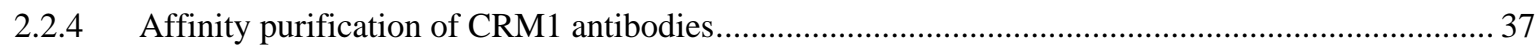

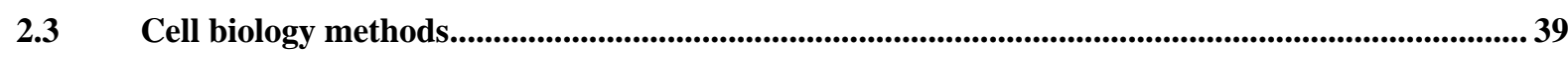

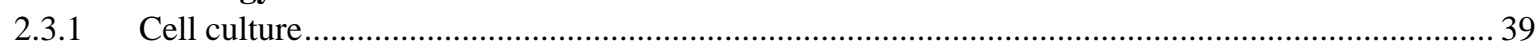

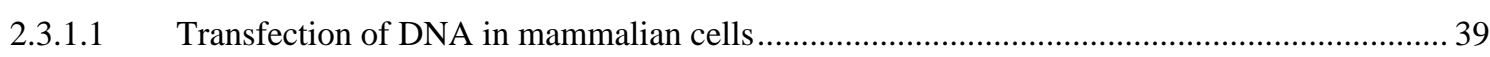

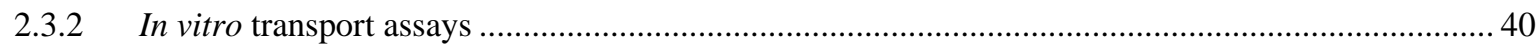

2.3.3 Detection of proteins by indirect immunofluorescence …………................................................. 41

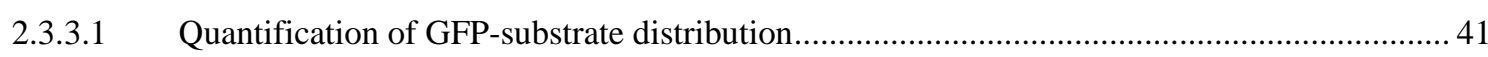




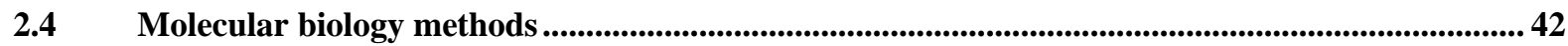

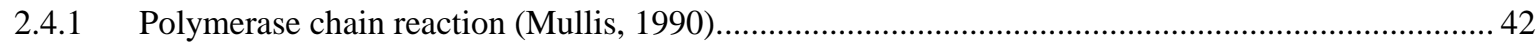

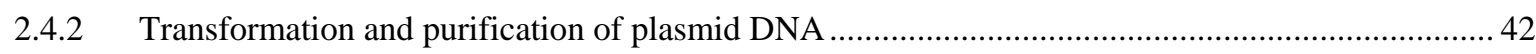

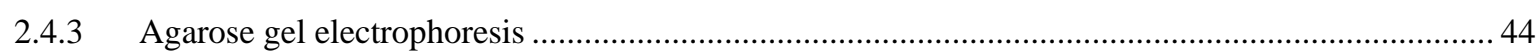

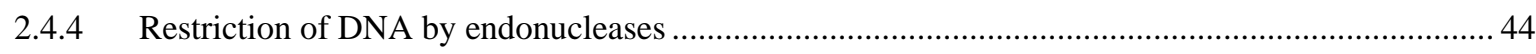

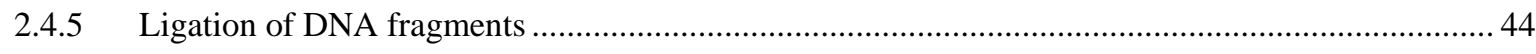

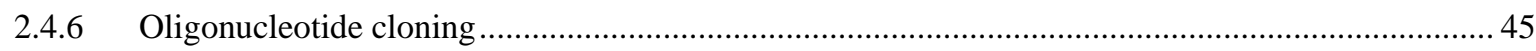

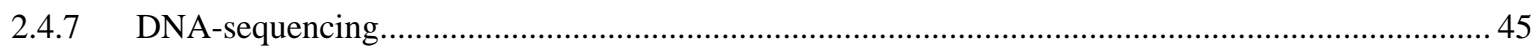

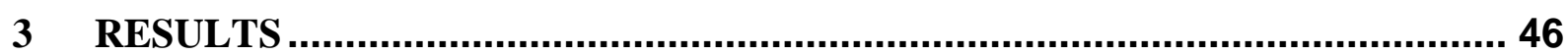

In vivo analysis of CRM1, CRM1-fragments and chimeras.........................................................46

3.1.1 Distribution of nuclear export cargos after Leptomycin B treatment of HeLa cells .......................47

3.1.2 The CRM1 mutant C528S can overcome the LMB effect on several export cargos .....................48

3.1.3 CRM1-fragments are not able to mediate nuclear export of proteins ...........................................50

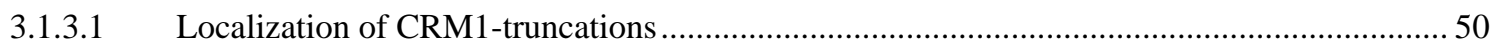

3.1.3.2 Only full-length CRM1 is able to mediate export through the nuclear pore ........................52

3.1.4 CRM1-chimeras as an alternative approach to study export capabilities in vivo ............................. 53

3.1.4.1 C. elegans CRM1 promotes export of a simple GFP-NES, but is unable to export more

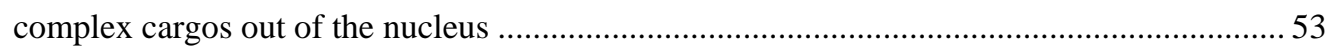

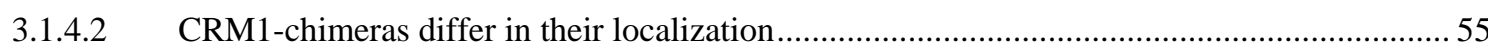

3.1.4.3 The export capability of CRM1-chimeras correlates with their localization in HeLa cells..... 57

3.1.4.4 The deletion of an outer surface loop between HEAT repeat 8B and 9A of CRM1 does not

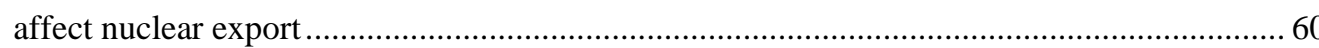

3.2 Determination of a minimal region in Nup214 required for CRM1-binding ..............................62

3.2.1 Function of Nup214 C-terminal fragments in nuclear export ..............................................62

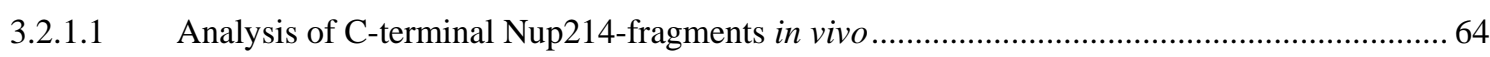

3.2.1.2 Accumulation of CRM1 in the nucleus upon overexpression of Nup214-fragments ............. 66

3.2.1.3 Nup214 1916-2033 shows an inhibitory effect on CRM1-dependent nuclear export of

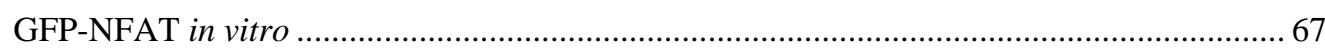

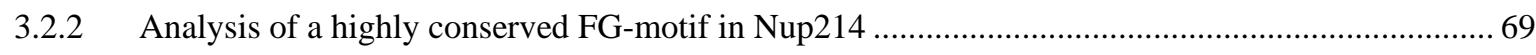

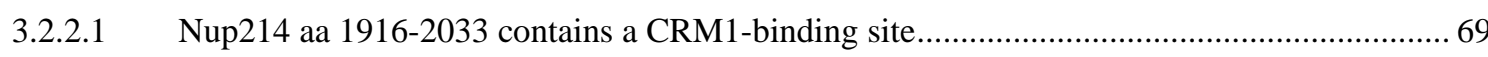

3.2.2.2 The mutation of the FGs in the FG-motif in Nup214 aa 1916-2033 leads to severe

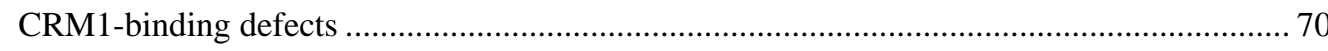

3.2.2.3 The inhibition of nuclear export is abrogated in Nup214 aa 1916-2033 a1 mutant in vivo

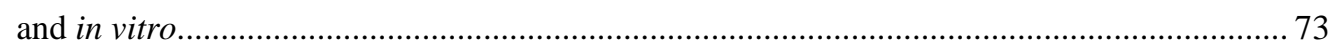

3.2.2.4 Analysis of the Nup214 FG-motif in Nup214 aa 1859-2090 ........................................... 75

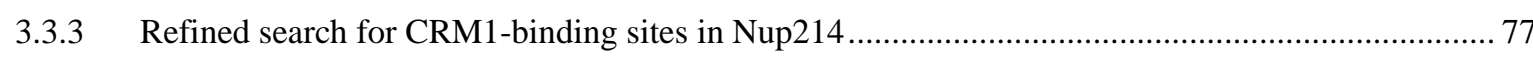

3.2.3.1 The FG-motif in Nup214 requires aa 1991-2033 to form a stable complex with CRM1

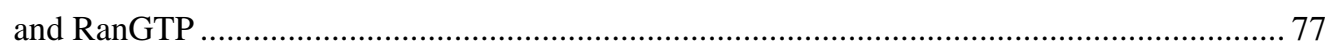

3.2.3.2 Elongation of Nup214 C-terminal fragments leads to increasing functionality in nuclear

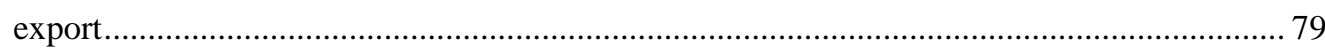




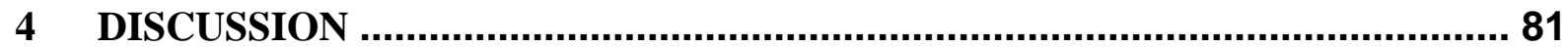

4.1 Analysis of CRM1-fragments and -chimeras ..........................................................................8 81

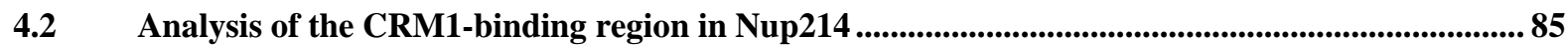

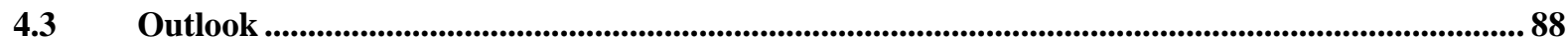

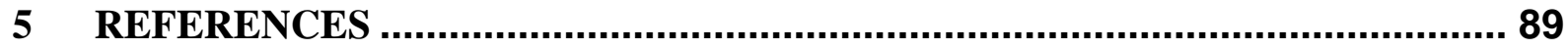

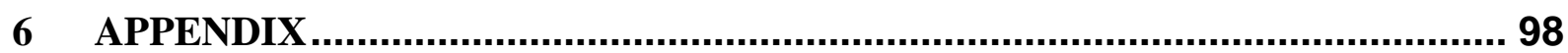

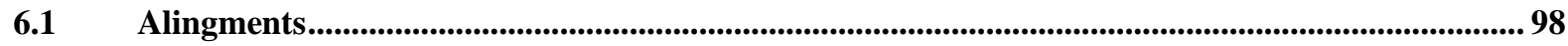

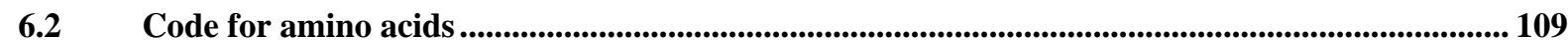

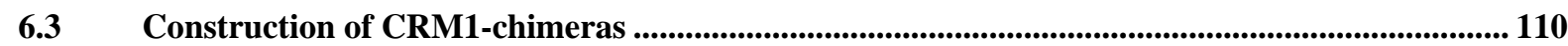

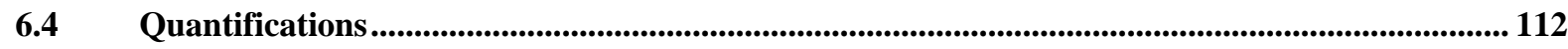

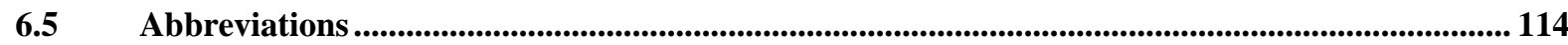

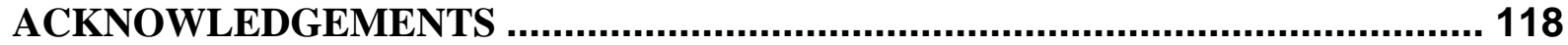

\section{CURRICULUM VITAE}




\section{Introduction}

In eukaryotic cells, a mechanism is required that allows transport of substrates between the nucleus and the cytoplasm according to the needs of the cell. The nuclear envelope is interspersed with special protein complexes. These complexes, named nuclear pore complexes (NPCs), provide the basis for directional transport to and out of the nucleus. All macromolecules that leave or enter the nucleus have to pass these complexes. These transport processes are strictly regulated so that most of the cargos cannot freely pass these NPCs. Transport receptors mediate their transport, thus ensuring the substrate specificity of nuclear entry and exit.

\subsection{The nuclear pore complex}

The NPC is a huge protein complex, embedded between the outer and inner nuclear membrane (Fig. 1A). NPCs have a calculated mass of 40-60 MDa in yeast (Rout et al., 2000, Cronshaw et al., 2002), and up to $125 \mathrm{MDa}$ in higher organisms (Reichelt et al., 1990). They allow bidirectional transport of proteins, RNAs and ribonucleoprotein (RNP) particles into and out of the nucleus (Stoffler et al., 1999; Conti and Izaurralde, 2001; Lei and Silver, 2002; Weis, 2002; reviewed in Wente and Rout, 2010). At the same time, NPCs generate a diffusion barrier to separate the nucleus from the cytoplasm. Passive diffusion is only possible for small metabolites and small proteins (Paine et al., 1975). Cryoelectron microscopy studies showed the NPC with an 8-fold rotational symmetry (Beck et al., 2004). In general, the NPC can be divided into three sections: the central part, anchored between the outer and the inner nuclear membrane, the cytoplasmic filaments and the nuclear spokes forming a basket (Fig. 1B, Hoelz et al., 2011). This structure is conserved from yeast to higher eukaryotes.

The NPC is built of approximately 30 proteins, named nucleoporins (Nups). They are present in the pore in copies of eight or multiples of eight (Cronshaw et al., 2002). The majority of Nups is symmetrically located on the nuclear and the cytoplasmic side of the NPC, but some of them occur only on either the nuclear or the cytoplasmic side like Nup358, Nup214 and Nup153 (reviewed in Hoelz et al., 2011). In general, Nups are primarily constructed from $\alpha$-solenoids, $\beta$-propellers, coiledcoil domains and transmembrane domains (Alber et al., 2007; Devos et al., 2006; Schwartz, 2005). Some of them are O-glycosylated, like Nup98, Nup214 and p62 (Holt et al., 1987; Snow et al., 1987). Four of the known Nups have been characterized as transmembrane proteins (Gp190, Gp210, Ncd1, Pom121) connecting the NPC with the nuclear envelope (Chadrin et al., 2010; Gerace et al., 1983; Hallberg et al., 1993; Mansfeld et al., 2006; Stavru et al., 2006). Nine Nups in vertebrates (and seven 
in yeast) act as coat proteins of the NPC (Lutzmann et al., 2002, 2005; Siniossoglou et al., 2000). Together, they form a subcomplex (Nup84 complex in yeast; Nup107-160 complex in human) and deletion of any of its members, e.g. Nup75, Nup107 and Nup160 (Siniossoglou et al., 1996) has severe effects on the architecture and the function of the NPC. The adaptor Nups (seven in yeast, and nine in vertebrates) associate with transmembrane Nups, coat proteins and also with the channel proteins (Fig. 1B; reviewed in Hoelz et al., 2011,). Interestingly, only the channel proteins, the Nups of the nuclear basket and the cytoplasmic filaments contain phenylalanine-glycine (FG) repeats, e.g. Nup358, p62, Nup153 or Nup214. These FG-repeat regions are unstructured and provide binding sites for transport receptors (Denning et al., 2003; Isgro and Schulten, 2007a/b; Macara, 2001; Peters, 2005). Typically, they are made up of 4-48 GLFG, FxFG, PxFG or SxFG, separated by spacers of variable length (Cronshaw et al., 2002; Rout et al., 2000).

(A)

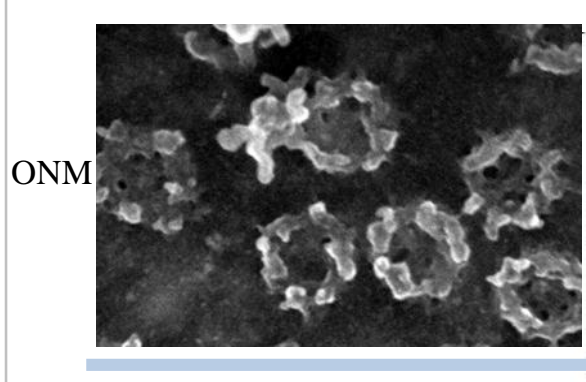

(B)

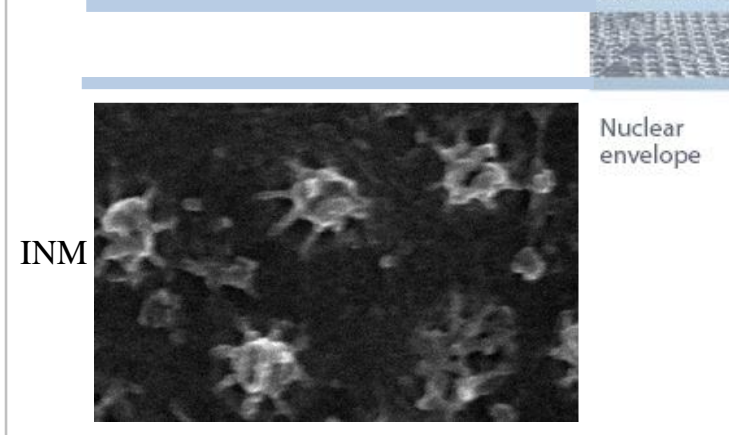

Nuclear basket

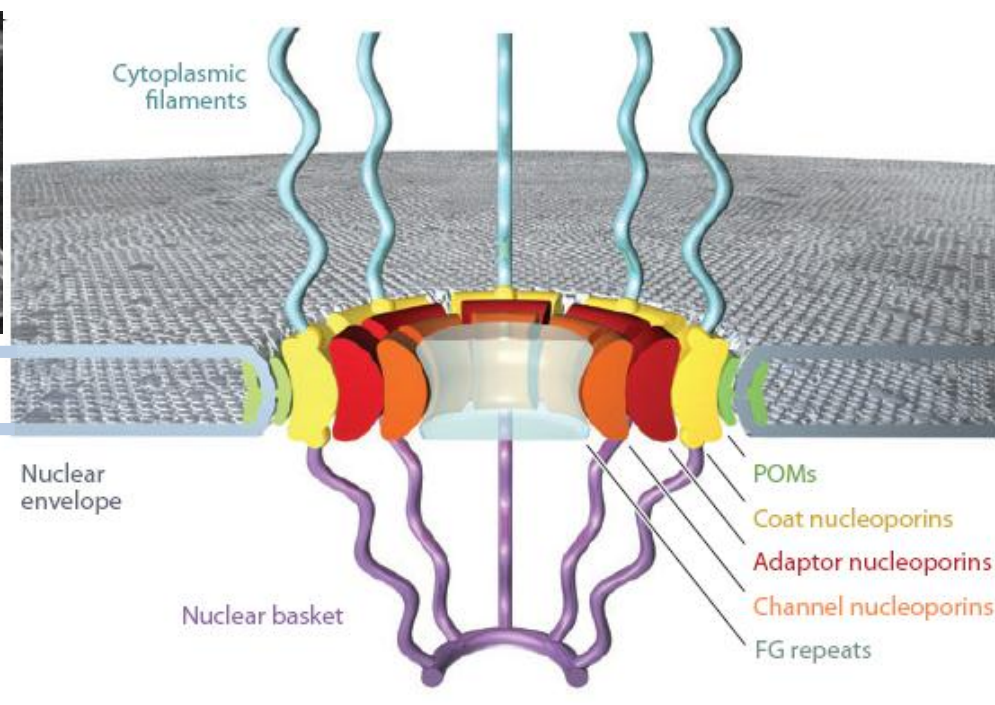

Fig. 1 The nuclear pore complex. (A) Pictures from the outer nuclear membrane (upper panel; ONM) and inner nuclear membrane (lower panel; INM) of Xenopus laevis oocytes, analyzed with field emission scanning electron microscopy (unpublished pictures, prepared in collaboration with the group of V. Cordes, MPI Göttingen). (B) Schematic model of the NPC, embedded in the nuclear envelope (Hoelz et al., 2011). 


\subsection{Transport receptors and regulators}

Most of the known transport receptors belong to the importin $\beta$ superfamily, also known as karyopherins (reviewed in Wente and Rout, 2010). They have a molecular mass of 90-145 KDa and are evolutionary conserved. Until today, approximately 20 different transport receptors are known. Depending on directionality of nuclear transport they are summarized as importins (transport of substrates into the nucleus) or exportins (transport of substrates out of the nucleus). They all consist of

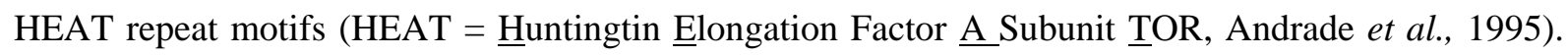
One HEAT repeat motif consists of two antiparallel $\alpha$-helices which are connected by a loop of variable length. This slightly clockwise twisted connection of up to 20 HEAT repeats leads to a superhelical structure of the whole protein (reviewed in Cook et al., 2007).

Active transport of proteins mediated by transport receptors is only possible if the cargo contains a recognition signal. This is either a nuclear localization signal (NLS), recognized by importins, or a nuclear export signal (NES), recognized by exportins.

Karyopherins do not only have a cargo recognition site, they also have binding domains for Nups (Bayliss et al., 2000/2002) and for the small GTPase Ran (Rexach and Blobel, 1995).

\subsubsection{The GTPase Ran}

The directionality of nuclear transport is regulated by the small GTPase Ran. It is a member of the Ras-related superfamily and besides its role in nuclear export it is also involved in mitotic processes in eukaryotes (reviewed in Clarke and Chuanmao, 2008). Transport receptors bind Ran only in its GTP bound form. This requires GDP-GTP conversion of Ran, mediated by accessory proteins which are located exclusively in either the nucleus or the cytoplasm (Fig. 2). In the nucleus, the chromatin bound RanGEF (Ran guanosine-nucleotide exchange factor), also called RCC1 (regulator of chromosome condensation 1), exchanges GDP to GTP (Bischoff and Postingl, 1991a). In the cytoplasm, the RanGTPase activating protein (RanGAP) stimulates the intrinsic GTPase activity of Ran (Becker $e t$ al., 1995; Bischoff et al., 1995; Seewald et al., 2002) with the help of the Ran binding protein 1 (RanBP1). RanGAP is a soluble cytoplasmic protein, but it is also associated with Nup358 (Mahajan et al. 1997; Matunis et al. 1996; Weis 2007), coordinating recycling of importin $\beta$ (Hutten et al., 2008). Due to cytoplasmic RanGAP and nuclear RCC1, there is a low concentration of RanGTP in the cytoplasm and a high concentration in the nucleus (Izaurralde et al., 1997). The nuclear transport factor 2 (NTF2) is closing the Ran-cycle, binding to RanGDP and mediating its import into the nucleus (Moore and Blobel, 1994; Ribbeck et al., 1998). 
In general, RanGTP decreases the affinity of import receptors for their import cargos, but increases the affinity of export receptors for their export proteins. Due to the high concentration of RanGTP in the nucleus, incoming import complexes dissociate, and formation of export complexes is promoted (reviewed in Wente and Rout, 2010).

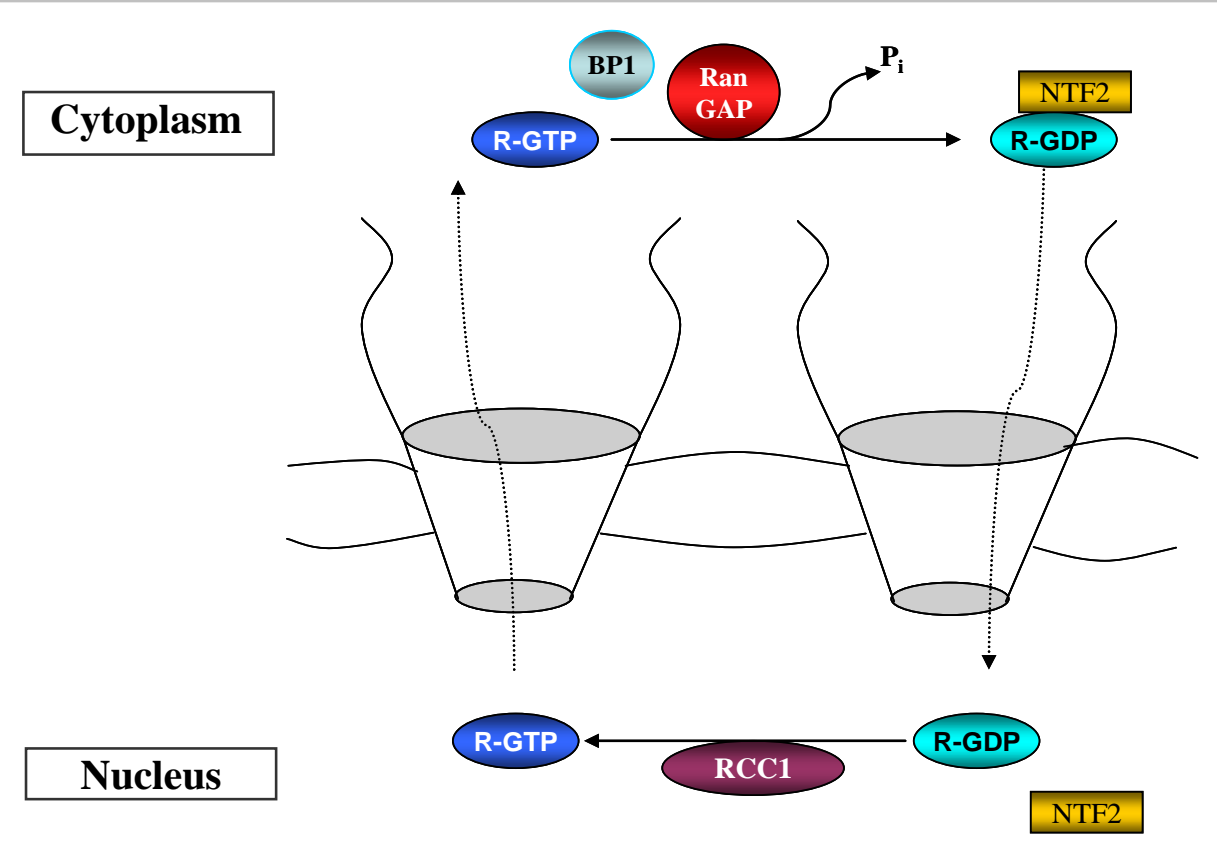

Fig. 2 The Ran-cycle. RanGTP, bound to an export receptor leaves the nucleus. In the cytoplasm, RanGAP and RanBP1 mediate hydrolysis of RanGTP to RanGDP. Bound to NTF2, RanGDP is transported back to the nucleus where RCC1 catalyzes the exchange of GTP to GDP.

\subsubsection{Basic nuclear import}

Nuclear import is mediated by a variety of import receptors. Among them, transportin, importin 5, 7, 9, 11 and 13 are receptors which are recognized by different import substrates. One of the best characterized import pathway is the importin $\alpha / \beta$ mediated import of cargos containing a classical NLS (cNLS). In general, the NLS-motif $\mathbf{K}(\mathbf{K} / \mathbf{R}) \mathbf{X}(\mathbf{K} / \mathbf{R})$ presents the required sequence for nuclear import of cargos by importin $\alpha / \beta$ (Conti et al., 2000; Fontes et al., 2000; Hodel et al., 2001). The cNLS can be monopartite (Kalderon et al., 1984), but many proteins also carry a more complex bipartite NLS consisting of two clusters of basic amino acids, which are separated by a spacer of about ten amino acids (Dingwall et al., 1982; Robbins et al., 1988). In the cytoplasm, importin $\alpha$ acts as an adaptor protein, recognizing the cNLS of the import-cargo and connecting it to importin $\beta$ via its IBB 
(importin $\beta$ binding) domain (Fig. 3; Görlich et al., 1995a, b). After passage of the import complex through the NPC, Ran binds to the outer convex surface of the import receptor, thereby displacing the import cargo from the inner concave surface of the receptor. Importin $\beta$, bound to RanGTP, is transported back to the cytoplasm (Rexach and Blobel, 1995). Nuclear export of importin $\alpha$ is mediated by the export receptor CAS (Kutay et al., 1997a).

Importin $\beta$ is known to interact with the FG-rich Nup358, the major component of the cytoplasmic filaments (Saitoh et al., 1996). In a current model, this interaction leads to enrichment of the import receptor at the nuclear pore and thus promoting nuclear import in mammalian cells (Hamada et al., 2011, Wälde et al., 2012).

Another well characterized import receptor is transportin. Instead of a cNLS, it recognizes substrates with a so called M9 sequence, which is glycine-rich and deficient in basic amino acids (Siomi et al., 1995; Nakielny et al., 1996). In crystal structure analysis, another transportin binding motif was identified, consisting of positively charged amino acids and a $\mathbf{R} / \mathbf{K} / \mathbf{H X}{ }_{(2-5)} / \mathbf{P Y}$-motif (Lee et al., 2006).

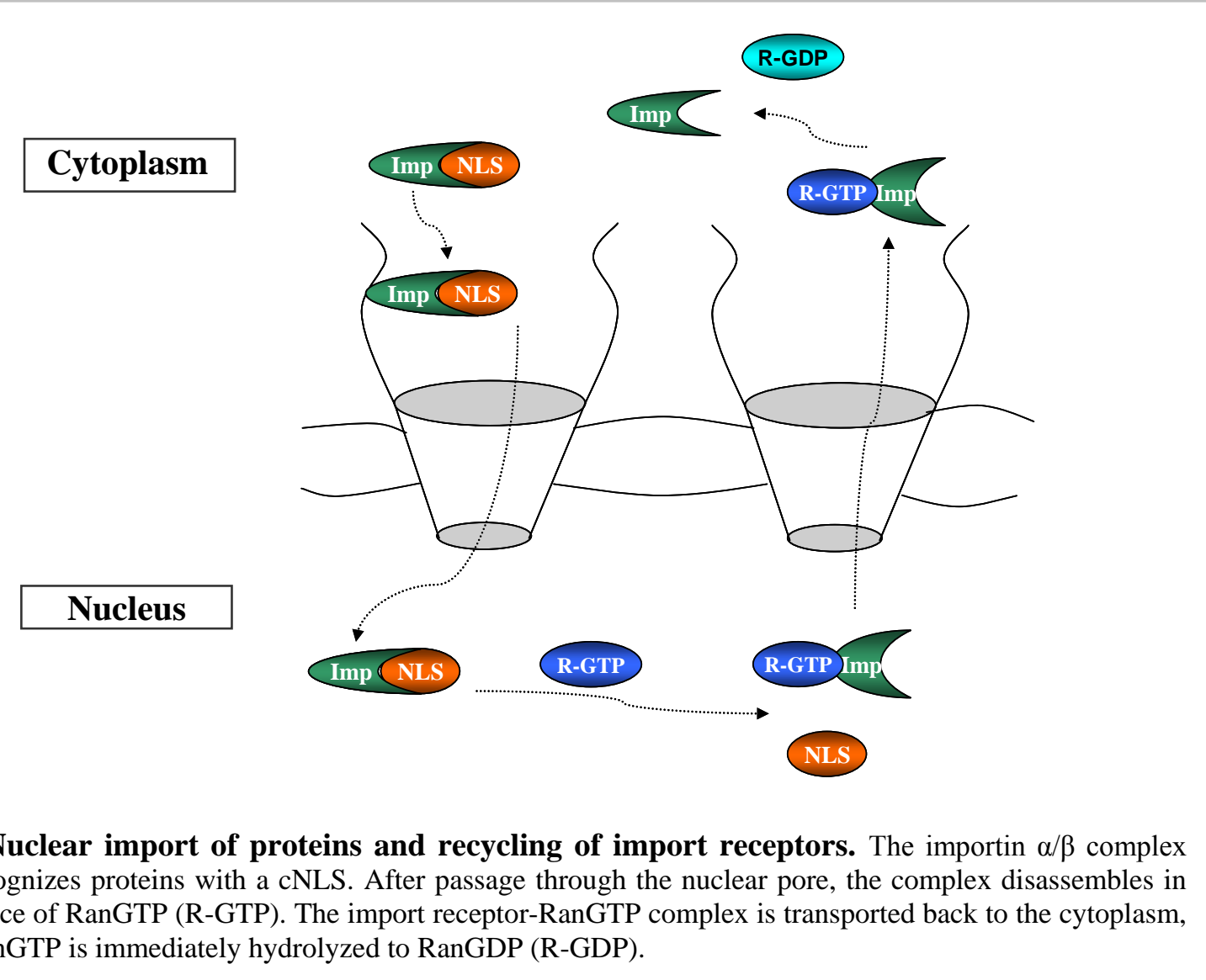




\subsubsection{Nuclear export}

So far, there are eight nuclear export receptors of the karyopherin family known (Table 1), and most of them act as transport receptors for specific substrates, e.g. CAS, which exclusively transports importin $\alpha$ to the cytoplasm. Besides transport of proteins, they also mediate transport of RNAs, either by direct interaction with RNA or with the help of adaptor proteins (Ohno et al., 2000; Arts et al., 1998; Kutay et al., 1998; reviewed in Wente and Rout, 2010). They also transport viral RNAs to the cytoplasm (reviewed in Carmody and Wente, 2009). The export receptor TAP, which forms a heterodimer with the cofactor p15 (Mex67/Mtr2 complex in yeast), exports mRNAs to the cytoplasm (Lutzmann et al., 2005; Santos-Rosa et al., 1998; Segref et al., 1997). TAP is an exception among transport receptors as it does not belong to the karyopherin family, but has cargo- and nucleoporinbinding sites and is shuttling between the nucleus and the cytoplasm. The TAP-p15 complex binds directly to the FG-repeats of the cytoplasmically located Nup214 (Fribourg et al., 2001).

\begin{tabular}{|c|c|}
\hline Export receptors & Substrates \\
\hline Exportin 1 (CRM1, Xpo1) & NES-Substrates (Fornerod et al., 1997b; Fukuda et al., 1997) \\
\hline CAS & Importin $\alpha$ (Kutay et al., 1997a) \\
\hline Exportin-t & tRNAs (Arts et al., 1998; Kutay et al., 1998) \\
\hline Exportin 4 & eIF5A (Lipowski et al., 2000); Smad3 (Kurisaki et al., 2006) \\
\hline Exportin 5 & microRNA precursors (Kim, 2004; Zheng and Cullen, 2004) \\
\hline Exportin 6 & Profilin, actin (Stüven et al., 2003) \\
\hline Exportin 7 & p50Rho-GAP, 14-3-36 (Mingot et al., 2004) \\
\hline Importin 13 & eIF-1 A (Mingot et al., 2001) \\
\hline
\end{tabular}

Table 1 List of known export receptors. (reviewed in Wente and Rout, 2010)

Like import receptors, also export receptors possess specific cargo recognition sites. These recognition sites are well studied for CRM1 (Chromosome region maintenance 1), the main export receptor in vertebrates and in yeast. CRM1 was initially discovered in Schizosaccharomyces pombe (Adachi and Yanagida, 1989) and it recognizes proteins with a so-called leucine-rich NES. The NES was originally identified in the HIV-Rev protein (Fischer et al., 1995) and the protein kinase inhibitor (Wen et al., 1995). The consensus sequence consists of hydrophobic amino acids $(\boldsymbol{\Phi})$ with a defined interval of other amino acids (X) between: $\Phi^{1} \mathbf{X}_{2-3} \Phi^{2} \mathbf{X}_{2-3} \Phi^{3} \mathbf{X} \Phi^{4}$. Classic NESs are commonly defined as leucine-rich NESs, but also isoleucine, valine, phenylalanine or methionine can be present at the hydrophobic positions in the consensus sequence (Bogerd et al., 1996; Zhang et al., 1998; Kosugi et al., 2008). 
Recent structural analysis of different NES peptides revealed a fifth hydrophobic amino acid being involved in CRM1 recognition ( $\underline{\Phi^{0}} \mathbf{X} \Phi^{1} \mathbf{X}_{2-3} \Phi^{2} \mathbf{X}_{2-3} \Phi^{3} \mathbf{X} \Phi^{4}$; Güttler et al., 2010). So far, several hundred cargos have been described to be exported via the NES in a CRM1-dependent manner (NESbase 1.0, la Cour et al., 2003).

\subsubsection{The structure of CRM1}

There is no structure available for CRM1 alone, but recently, the structure of CRM1 together with an export cargo and RanGTP has been solved (Dong et al., 2009; Monecke et al., 2009). There, CRM1 consists of 21 HEAT (HR) repeat motifs which are arranged in a ring-like structure (Fig. 4). RanGTP is present in the middle of this structure and is associated to CRM1 via many binding sites. The most prominent Ran interaction surface is an acidic loop between HR 9A and 9B, but also regions within HR 1-4, 7, 8, 13, 15, 17, 18 and 19 interact with RanGTP. Snurportin 1 (SPN1), which is shown as an export cargo, is an $\mathrm{m}_{3} \mathrm{G}$-cap receptor and imports $\mathrm{m}_{3} \mathrm{G}$-capped uridine-rich small nuclear RNPs (U snRNPs; Huber et al., 1998). SPN1 is recycled back to the cytoplasm with the help of CRM1 (Paraskeva et al., 1999). It binds via its NES to a hydrophobic cleft of CRM1 that is built by HR 11A and $12 \mathrm{~A}$ and additionally interacts with HR 14A, 15A and 16A.

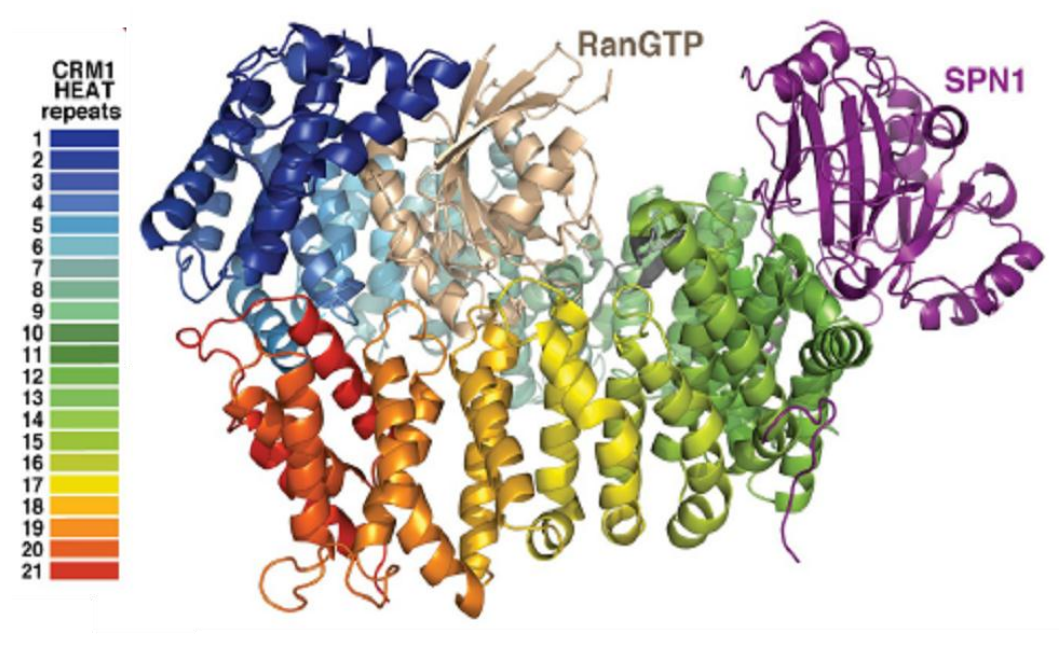

Fig. 4 Crystal structure of the trimeric export complex. CRM1 is a ring-like protein (shown in rainbow-colours; blue - N-terminus, red - C-terminus). RanGTP (light brown-coloured) binds to the inner concave surface of CRM1, interacting with several HEAT repeats. The export cargo SPN1 (lilaccoloured) binds to the outer convex surface of CRM1 (picture taken from Monecke et al., 2009). 
As there are many more CRM1-dependent export cargos with different globular structures, there is no unique binding mechanism, but all of these cargo proteins have a NES in common. Although the NES differs from protein to protein (Güttler et al., 2010), it exclusively binds to the hydrophobic cleft of CRM1. Because of that, CRM1-mediated nuclear export can be selectively inhibited by Leptomycin B (LMB). LMB, an unsaturated fatty acid, covalently modifies the cysteine 528 in CRM1 (Kudo et al., 1999), which is present in the hydrophobic cleft. As a consequence, export cargos cannot bind to LMB-modified CRM1 with their NES, and the formation of a functional export complex is prevented.

\subsubsection{CRM1-mediated nuclear export}

CRM1 mediates export of various NES-cargos, but also other substrates like the 60S preribosomal subunit (Gadal et al., 2001) or several RNAs (rRNAs, U snRNAs, viral mRNAs and some cellular mRNAs; Johnson et al., 2002; Kimura et al., 2004; Zeiner et al., 2003; reviewed in Siddiqui and Borden, 2012). There, CRM1 does not interact directly with RNAs, but uses adaptor proteins containing an NES (reviewed in Rodriguez et al., 2004). In general, NES-cargos have a very low affinity to CRM1, even in the presence of RanGTP (Askjaer et al., 1999). SPN1, which is shown above in the crystal structure, is an exception, as it can bind to CRM1 even in the absence of RanGTP (Paraskeva et al., 1999). To date, it remains unclear whether the presence of RanGTP influences the conformation of CRM1. A model (Monecke et al., 2009) suggests a relaxed conformation of CRM1 in the cytoplasm and a strained conformation in the nucleus, thus modulating the accessibility of the hydrophobic cleft for export cargos. On the other hand, X-ray scattering and electron microscopy studies suggest that the conformation of CRM1 does not change (Dong et al., 2009; Fukuhara et al., 2004; Petosa et al., 2004).

Basically, the trimeric export complex is a result of cooperative binding of RanGTP and the export cargo to CRM1 (Fig. 5; Fornerod et al., 1997b). The formation of this complex seems to be the ratelimiting factor as nuclear export is stimulated in the presence of a Ran mutant with increased affinity to CRM1 (Kehlenbach et al., 2001). Recent studies showed that the very C-terminal part of human CRM1 consists of a short $\alpha$-helix containing four acidic amino acid residues which modulate the affinity of CRM1 to NES-cargos (Dong et al., 2009; Fox et al., 2011). This helix adopts different positions in the CRM1 molecule depending on RanGTP presence or absence, thus providing a further regulatory mechanism of cargo binding.

Once bound to RanGTP, the HEAT 9 loop in CRM1 becomes an ordered $\beta$-hairpin, interacting with HRs 12-15. Together with the C-terminal acidic $\alpha$-helix, the HEAT 9 loop plays a central role in modulation of NES-cargo binding (Fox et al., 2011). The Ran binding protein 3 (RanBP3) supports the complex formation by providing sufficient amounts of RanGTP (Müller et al., 1998; Nemergut et 
al., 2002). Additionally, it increases the affinity of CRM1 for RanGTP in the trimeric export complex, finally resulting in a stable quaternary complex with the ability to interact with Nups (Engelmeier $e t$ al., 2001; Lindsay et al., 2001). Recently, it has been shown that the mobile FG-containing nucleoporin Nup98 interacts with CRM1 in a RanGTP-dependent manner (Oka et al., 2010), and RanBP3 regulates this interaction. Furthermore, also the nucleoporin-like protein 1 (NLP1; Waldmann et al., 2012) promotes nuclear export of the CRM1-complex, functioning as an accessory factor.

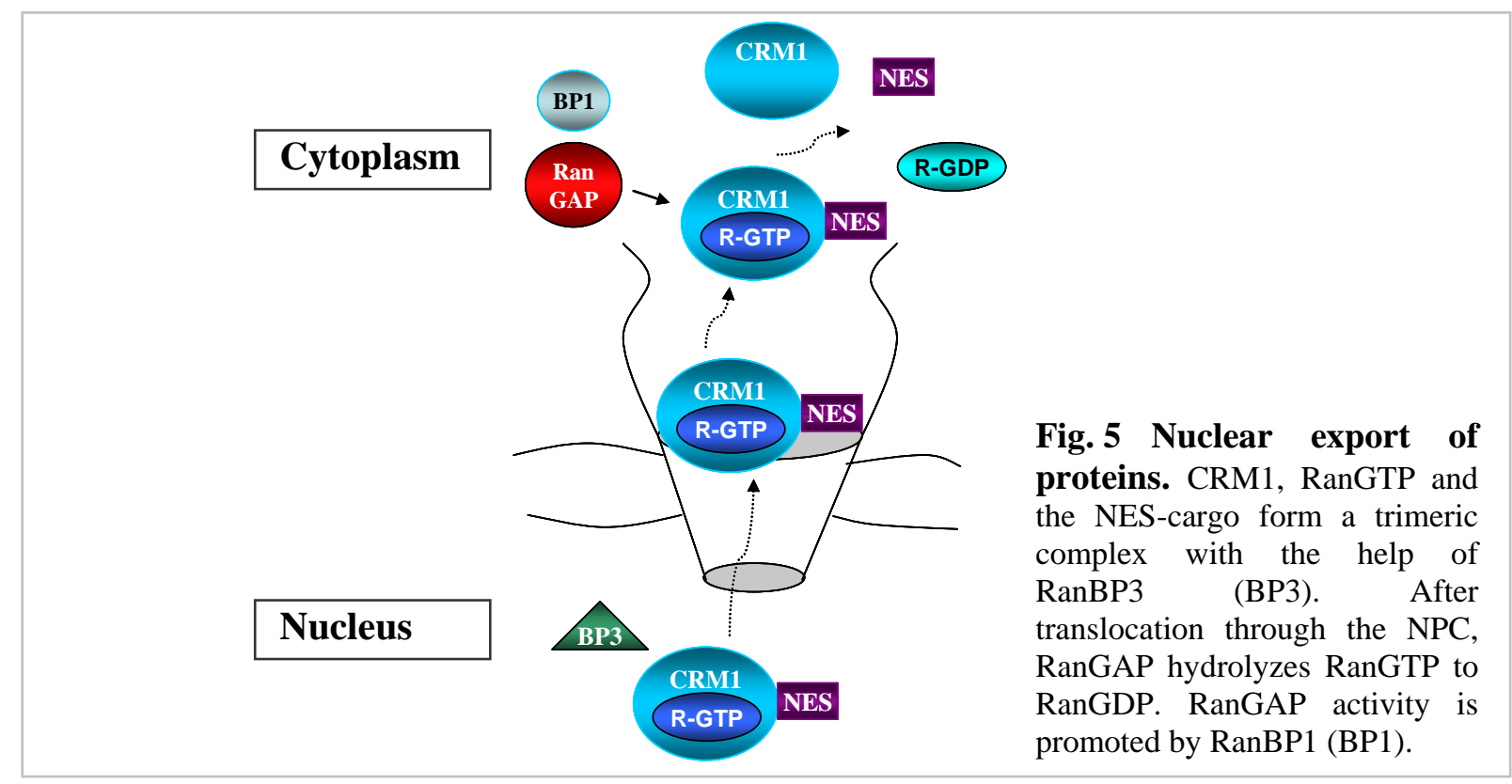

The process of translocation of the export complex through the nuclear pore has not been completely resolved yet, but CRM1 is known to interact with several Nups like Nup153, p62 and Nup214/Nup88 (Hutten and Kehlenbach, 2006; Nakielny et al., 1999) in a RanGTP-dependent manner. In the cytoplasm, the trimeric export complex is released with RanBP1 and RanGAP. RanBP1 prepares the export complex to hydrolysis of RanGTP, as crystal structure comparison of the CRM1-RanGTP-NES complex with the CRM1-RanGTP-RanBP1 complex revealed an allosteric mechanism of RanGTP displacement, involving the HEAT 9 loop of CRM1 (Koyama and Matsuura, 2010). The recycling of CRM1 back to the nucleus is poorly understood. Nup358, the major component of the cytoplasmic filaments of the NPC, has been shown to interact with CRM1 (Singh et al., 1999), but it is discussed whether this interaction plays a role in the late steps of export complex disassembly or whether it is already the initial binding site for retransport of unbound CRM1 to the nucleus (Bernad et al., 2004; Hutten and Kehlenbach, 2006). 


\subsection{The translocation of transport complexes through the nuclear pore}

To date, it is not exactly known how transport complexes translocate through the nuclear pore. Several studies showed that transport receptors interact with FG-rich Nups (Bayliss et al., 1999/2000/2002; Fornerod et al, 1997a; Fribourg et al., 2001; Saitoh et al., 1996). Generally, it is believed that this interaction occurs via the FGs of Nups. Based on this, several models exist, which explain the efficient passage of transport receptor-cargo complexes through the nuclear pore.

In the oily-spaghetti-model (Macara, 2001), the NPC is an open structure, in which FG containing repeat Nups behave like loose "oily spaghetti". The open central channel has a diameter of $10 \mathrm{~nm}$ (Keminer and Peters, 1999). The FG-spaghetti would form an approximately $7 \mathrm{~nm}$ layer around this tube. Transport receptors are able to interact with the FGs, moving randomly within the nuclear pore for a short distance. This "hopping" from FG to FG results in a facilitated diffusive movement through the nuclear pore. The diameter of the central tube would be big enough to allow sufficient translocation of many transport complexes at the same time.

The selective phase-model (Ribbeck and Görlich, 2001) suggests that FG-rich Nups form a meshwork in the central channel of the NPC, providing a permeability barrier for molecules. Transport receptors interact with the FGs, thus becoming part of the meshwork. Recent studies showed that complexes containing transport receptor domains, which can interact with FGs, can translocate extremely fast into an FG-hydrogel, whereas the passage of molecules without this receptor domain is clearly restricted (Frey and Görlich, 2006/2007).

The "virtual gate"-model considers entropic factors of macromolecules (Rout et al., 2003). In general, molecules having a defined energy state can move freely in the cell. The NPC restricts this movement, because the FG-Nups form a dense meshwork in the channel. The molecules require a higher energy state to reach a "transition state", which enables them to cross the NPC. Small molecules can afford this energy, but for bigger molecules the energy would be too high. Transport receptors bind to FGNups, which results in decrease of the energy costs. Thus, transport receptors can reach the "transition state" and move through the nuclear pore.

The forest-model combines the selective phase- and the virtual gate- model (Yamada et al., 2010). Additionally, Nups are categorized in different structures. The analysis of topology, dimensions, stoichiometry, interactivity and approximate anchoring of the FG-Nups to the wall of NPC leads to a "forest"-like landscape of the nuclear pore channel. FG-Nup domains can either occur as extended, relaxed or collapsed coils, where the relaxed and extended coils are more dynamic than the collapsed coils. Depending on the charge of these domains, they are more attractive (low charge content) or repulsive (high charge content). Certain Nups only have collapsed coils, therefore looking like a "shrub". Other Nups are anchored to the wall of the NPC via extended coils and additionally can have collapsed coils sitting on the extended coil, resulting in a "tree"-like structure. The "trees" extend to the centre of the NPC channel, thereby creating a "transporter" zone (zone 1). There, transport 
receptors with large substrates can move. The "shrubs" do not extend to the centre of the channel, but create another zone (zone 2) near the conduit of the NPC, where small molecules and transport receptors with small substrates can translocate. In this model, zone 1 functions as a permeability barrier, as described by the selective phase-model, and zone 2 allows passage of molecules with corresponding loss of entropic energy as described in the "virtual gate"-model.

\subsection{Nup214}

Human Nup214, also known as CAN, was firstly identified in leukemia-associated chromosomal translocations involving the SET and DEK genes (von Lindern et al., 1992a, b). In T-cells of patients suffering from acute lymphoblastic leukaemia, a third translocation was found, which generates a fusion between Nup214 and the constitutive tyrosine kinase ABL (Abelson murine leukemia viral oncogene homolog 1; Grauxl et al., 2004). The activity of the Nup214-ABL fusion is dependent on its tethering to the nuclear pore complex (de Keersmaecker et al., 2008).

Nup214 is an essential nucleoporin, as depletion in mice leads to strong mRNA export defects and to lethality (van Deursen et al., 1996). It is located at the cytoplasmic side of the NPC (Kraemer et al., 1994), and transmission electron microscopy (TEM) and field emission in-lense scanning electron microscopy (FEISEM) studies revealed that Nup214 is located near the central channel of the pore (Walther et al., 2002). Nup214 (see Fig. 6) contains an N-terminal $\beta$-propeller, which recruits the DDX19 helicase (Moeller et al., 2009; Napetschnig et al., 2006/2009). The central coiled-coil domains mediate the interaction with the anchor protein Nup88 (Bastos et al., 1997; Fornerod et al., 1997a), resulting in a subcomplex.

Fig. 6 Schematic view of human Nup214. The numbers indicate the amino acid residues of
domains and regions. The structure of the $\mathrm{N}$-terminus was determined by crystal structure analysis
(Weirich $e$ t al., 2004, Napetschnig $e$ al al., 2006), the coiled-coil domains in the central regions are
predicted. The C-terminal part of Nup214 is believed to be unstructured.


Deletion of Nup88 leads to a loss of Nup214 at the nuclear pore, and vice versa (Bernad et al., 2004; Hutten and Kehlenbach, 2006). By contrast, other Nups like p62, Nup153 or Nup358 are not affected upon Nup214 depletion (Hutten and Kehlenbach, 2006).

The FG-rich region in the C-terminus of Nup214 was identified as the binding site for CRM1, and this binding is dependent on RanGTP (Fornerod et al., 1996). Furthermore, this binding is increased in the presence of an export substrate (Kehlenbach et al., 1999). Nup214 is not only associated to CRM1mediated export, it is also involved in nuclear import of certain molecules. Nup214 exposes a docking site for nucleocapsids of large viruses and facilitates their import into the nucleus independent of transport receptors (Trotman et al., 2001). Furthermore, Nup214 has been shown to play a role in nuclear import of the transcription factors Smad2 (Xu et al., 2002), Stat1 (Marg et al., 2004) and NF-кB (Xylourgidis et al., 2006).

The role of the CRM1-Nup214 interaction is highly discussed. The lack of Nup88 causes mislocalization of CRM1 (Roth et al., 2003), and it is suggested that the Nup214-88 complex is the terminal docking site for CRM1 in nuclear export in vitro (Fornerod et al., 1996; Kehlenbach et al., 1999). Strikingly, only a few substrates have been identified, which are Nup214- and CRM1dependent. Nup214 depletion leads to impaired mediated nuclear export of NFAT (nuclear factor of activated T-cells), the viral protein HIV-Rev (Hutten and Kehlenbach, 2006) and the 60S preribosomal subunit via the adaptor protein Nmd3 (Bernad et al., 2006). In yeast, the C-terminus of Nup214 is not required at all for CRM1-dependent nuclear export of shuttling reporter proteins (Zeitler and Weis, 2004).

\subsection{Aim of the work}

The strong binding of the FG-containing Nup214 C-terminus to CRM1 suggests a general role for Nup214 in nuclear export of proteins. Whereas the region at the C-terminal end of Nup214 is known to bind CRM1, the region(s) in CRM1 for interaction with Nup214 (and other Nups) has not been identified so far. The identification of these sites would help to elucidate the role of CRM1nucleoporin interaction, e.g. for translocation of export complexes through the nuclear pore or their disassembly in the cytoplasm. For this reason, we perform microscopic studies with CRM1 fragments and chimeras and investigate their association with the nuclear envelope and their functionality in nuclear export.

The FG-containing Nup214 region that binds to CRM1 comprises 230 amino acids. It is known for other karyopherins that they bind to the FGs in nucleoporins, but for CRM1 this can only be assumed. With Nup214 FG-mutants, we want to investigate whether the FGs in Nup214 are responsible for CRM1 interaction. To narrow down the CRM1-binding region in Nup214, we combine binding studies with in vivo assays, using short Nup214 fragments. 


\section{Material and methods}

\subsection{Material}

\subsubsection{Technical equipment and software}

\begin{tabular}{|c|c|}
\hline Technical equipment & Company \\
\hline Centrifuge Avanti J-30I & Beckman Coulter \\
\hline Documentation system LAS 3000 & Fujifilm \\
\hline Emulsi flex-C5 & Avestin \\
\hline FACS Canto $^{\mathrm{TM}} \mathrm{II}$ & BD Biosciences \\
\hline Film developing machine Curix 60 & AGFA \\
\hline Fluorescence microscope Axioskop 2 & Zeiss \\
\hline Rotors JA 30.50Ti, SX4750, TLA 100.3 & Beckman Coulter \\
\hline SE260 Small Format Vertical Electrophoresis & \\
\hline System & Hoefer \\
\hline Sequencer, Genetic Analyser 3100 & Applied Biosystems \\
\hline Table centrifuges 5415 D, 5415 R, 5424 & Eppendorf \\
\hline Thermocycler Gene Amp PCR 2400 & Perkin Elmer \\
\hline Thermocycler Primus MWG & Biotech \\
\hline Thermocycler Tprofessional Gradient & Biometra \\
\hline Thermocycler Tprofessional Standard & Biometra \\
\hline Ultracentrifuge OptimaMax & Beckman Coulter \\
\hline
\end{tabular}




\begin{tabular}{l|l}
\hline Software & Company/Origin \\
\hline AxioVision (LE) Rel. 4.5 & Carl Zeiss \\
BLAST & http://blast.ncbi.nlm.nih.gov/ \\
Chromas Lite 2.01 & Technelysium \\
Image Reader LAS 3000 & Fuji \\
Ligation calculator & http://www.insilico.uni-duesseldorf.de/Lig_Input.html \\
Oligonucleotide properties calculator & http://www.basic.northwestern.edu/biotools/ \\
& oligocalc.html \\
Photoshop 6.0 & Adobe \\
Vector NTI & Invitrogen
\end{tabular}

\subsubsection{Consumables}

\section{Consumables}

Autoradiography films

Cell culture consumables

Centrifugal filter units

FACS sample tubes

Microscope slides (76x26x1 mm)

Microscope cover slips 1.0 Borosilicate

Parafilm

Polystyrene columns

PROTRAN nitrocellulose

Sterile filters and membranes

$(0.22-0.45 \mu \mathrm{M})$

Syringes and needles

\section{Company}

GE Healthcare, Kodak

Sarstedt, TPP

Millipore, Vivaspin

BD Biosciences

Marienfeld

Marienfeld

Pechiney Plastic packaging

Thermo Scientific

Schleicher and Schuell

Millipore, Pall, Renner, Sartorius

Braun 


\subsubsection{Kits}

\begin{tabular}{l|l}
\hline Kits & Company \\
\hline BigDye Terminator v1.1 cycle sequencing kit & Applied Biosystems \\
NucleoBond $^{\circledR}$ PC100, PC500 & MACHEREY-NAGEL \\
NucleoSpin $^{\circledR}$ Extract II & MACHEREY-NAGEL
\end{tabular}

\subsubsection{Chemicals, reagents and enzymes}

Standard chemicals and reagents were obtained from AppliChem (Darmstadt), FLUKA (Buchs, Switzerland), Merck (Darmstadt), Carl Roth (Karlsruhe), SERVA (Heidelberg) and Sigma Aldrich (Taufkirchen). Specific reagents are listed below.

\begin{tabular}{l|l}
\hline Reagent & Origin \\
\hline acrylamide (30\%) & Amersham \\
aprotinin & Biomol \\
ATP & Sigma Aldrich \\
BSA, fraction V & PAA \\
calf intestinal phosphatise & Fermentas \\
Immobilon ${ }^{\text {TM }}$ Western & Millipore \\
creatine phosphate & Calbiochem \\
creatine phosphate kinase & Calbiochem \\
Cyanogen bromide-activated Sepharose ${ }^{\circledR} 4 \mathrm{~B}$ & Sigma Aldrich \\
digitonin & Calbiochem \\
dimethylsulfoxide & Applichem \\
dithiothreitol & AppliChem \\
DMEM (high glucose) & Gibco \\
DNA ladder, 1kb & Fermentas \\
dNTPs & Fermentas \\
FCS & Gibco \\
Fluorescent mounting medium & Dako \\
Freundsches Adjuvant incomplete & Sigma Aldrich \\
\hline
\end{tabular}




\begin{tabular}{|c|c|}
\hline Reagent & Origin \\
\hline glutamine & Gibco \\
\hline GDP, GTP & Sigma Aldrich \\
\hline Glutathione sepharose ${ }^{\mathrm{TM}}$ High Performance & GE Healthcare \\
\hline Hoechst 33258 & Sigma Aldrich \\
\hline ionomycin & Sigma Aldrich \\
\hline IPTG & Fermentas \\
\hline leptomycin B & Alexis Biochemicals \\
\hline leupeptin & Biomol \\
\hline Ni-NTA agarose & Qiagen \\
\hline penicillin & PAA \\
\hline pepstatin & Biomol \\
\hline Phusion 530L Polymerase & New England Biolabs \\
\hline PMSF & Sigma Aldrich \\
\hline poly-L-Lysine & Sigma Aldrich \\
\hline protein assay reagent & BIO-RAD \\
\hline protein ladder PAGE ruler & Fermentas \\
\hline restriction enzymes & Fermentas \\
\hline RNase A & Applichem \\
\hline SERVA DNA stain G & SERVA \\
\hline sequencing mix and buffer & Applied Biosystems \\
\hline siRNA oligonucleotides & Applied Biosystems \\
\hline streptomycin & PAA \\
\hline T4 DNA Ligase & Fermentas \\
\hline $\mathrm{T} 4$ polynucleotide kinase & Fermentas \\
\hline Titermax ${ }^{\circledR}$ Gold & Sigma Aldrich \\
\hline trichostatin A & Sigma Aldrich \\
\hline trypane blue & Fluka \\
\hline trypsin & Gibco \\
\hline Vent polymerase & New England Biolabs \\
\hline WGA & Sigma Aldrich \\
\hline
\end{tabular}




\subsubsection{Buffers, stock solutions and media}

All buffers and media were prepared using deionized water and autoclaved at $121{ }^{\circ} \mathrm{C}$ for 20 minutes. Buffers were titrated with $\mathrm{NaOH}, \mathrm{HCL}$ or $\mathrm{KOH}$ (only for HEPES buffers). Solutions with heatsensitive substances were sterilized using a $0.2 \mu \mathrm{m}$ sterile filter.

\begin{tabular}{|c|c|}
\hline Buffers & \\
\hline annealing buffer $(2 x)$ & $\begin{array}{l}100 \mathrm{mM} \text { potassium acetate, } 30 \mathrm{mM} \text { HEPES } \mathrm{pH} \text { 7.4, } 2 \mathrm{mM} \\
\text { magnesium acetate }\end{array}$ \\
\hline colloidal coomassie dye & $\begin{array}{l}0.1 \%(\mathrm{v} / \mathrm{v}) \text { brilliant blue G-250, } 2 \%(\mathrm{w} / \mathrm{v}) \text { ortho-phosphoric } \\
\text { acid, } 10 \%(\mathrm{w} / \mathrm{v}) \text { ammonium sulphate, } 20 \% \text { methanol }\end{array}$ \\
\hline colloidal coomassie fixing solution & $40 \%(\mathrm{v} / \mathrm{v})$ ethanol, $10 \%(\mathrm{v} / \mathrm{v})$ acetic acid \\
\hline coomassie dye & $10 \%(\mathrm{v} / \mathrm{v})$ acetic acid, $0.025 \%(\mathrm{w} / \mathrm{v})$ brilliant blue G-250 \\
\hline coomassie fixing solution & $45 \%(\mathrm{v} / \mathrm{v})$ methanol, $10 \%(\mathrm{v} / \mathrm{v})$ acetic acid \\
\hline 10x DNA loading dye & $\begin{array}{l}50 \%(\mathrm{v} / \mathrm{v}) \text { glycerol, } 0.1 \%(\mathrm{w} / \mathrm{v}) \text { bromphenol blue, } 0.1 \%(\mathrm{w} / \mathrm{v}) \\
\text { xylencyanol }\end{array}$ \\
\hline GST buffer & $\begin{array}{l}50 \mathrm{mM} \text { Tris- } \mathrm{HCl} \text { (pH 6.8), } 300 \mathrm{mM} \mathrm{NaCl}, 0.25 \mathrm{mM} \text { EDTA, } \\
1 \mathrm{mM} \mathrm{MgCl} 2 \text {, aprotinin, leupeptin, pepstatin, }(1 \mu \mathrm{g} / \mathrm{ml} \text { each), } \\
0.1 \mathrm{mM} \mathrm{PMSF}\end{array}$ \\
\hline 2x HEPES buffered saline (HBS) & $50 \mathrm{mM}$ HEPES (pH 6.98), $250 \mathrm{mM} \mathrm{NaCl}, 1.5 \mathrm{mM} \mathrm{Na}_{2} \mathrm{HPO}_{4}$ \\
\hline 10x Laemmli running buffer & $250 \mathrm{mM}$ Tris, $1.92 \mathrm{M}$ glycine, $0.1 \%$ (w/v) SDS \\
\hline Nup214 buffer & $\begin{array}{l}50 \mathrm{mM} \text { Tris- } \mathrm{HCl}(\mathrm{pH} 6.8), 300 \mathrm{mM} \mathrm{NaCl}, 10 \% \text { glycerol, } \\
4 \mathrm{mM} \beta \text {-mercaptoethanol, } 1 \mathrm{mM} \mathrm{MgCl} 2 \text {, aprotinin, leupeptin, } \\
\text { pepstatin, }(1 \mu \mathrm{g} / \mathrm{ml} \text { each), } 0.1 \mathrm{mM} \mathrm{PMSF}\end{array}$ \\
\hline P1 buffer & $50 \mathrm{mM}$ Tris- $\mathrm{HCl}(\mathrm{pH} 8), 10 \mathrm{mM}$ EDTA, $100 \mu \mathrm{g} / \mathrm{ml}$ RNase A \\
\hline P2 buffer & $200 \mathrm{mM} \mathrm{NaOH}, 1 \%(\mathrm{v} / \mathrm{v}) \mathrm{SDS}$ \\
\hline P3 buffer & $3 \mathrm{M} \mathrm{KOAc}(\mathrm{pH} 5.3)$ \\
\hline
\end{tabular}




\begin{tabular}{|c|c|}
\hline$\underline{\text { Buffers }}$ & \\
\hline phosphate buffered saline (PBS) & $\begin{array}{l}140 \mathrm{mM} \mathrm{NaCl}, 2.5 \mathrm{mM} \mathrm{KCl}, 10 \mathrm{mM} \mathrm{Na}{ }_{2} \mathrm{HPO}_{4}, \mathrm{KH}_{2} \mathrm{PO}_{4} \\
(\mathrm{pH} 7.5)\end{array}$ \\
\hline PBS Tween & $\begin{array}{l}140 \mathrm{mM} \mathrm{NaCl}, 2.5 \mathrm{mM} \mathrm{KCl}, 10 \mathrm{mM} \mathrm{Na}_{2} \mathrm{HPO}_{4}, \\
\mathrm{KH}_{2} \mathrm{PO}_{4}(\mathrm{pH} 7.5)+0.1 \%(\mathrm{v} / \mathrm{v}) \text { Tween } 20\end{array}$ \\
\hline Ponceau S & $0.5 \%(\mathrm{w} / \mathrm{v})$ Ponceau S, $1 \%(\mathrm{v} / \mathrm{v})$ acetic acid \\
\hline SDS-PAGE loading buffer $(4 \mathrm{x})$ & $\begin{array}{l}4 \%(\mathrm{w} / \mathrm{v}) \mathrm{SDS}, 125 \mathrm{mM} \text { Tris } \mathrm{pH} 6.8,10 \%(\mathrm{v} / \mathrm{v}) \text { glycerol, } \\
0.02 \%(\mathrm{w} / \mathrm{v}) \text { bromphenol blue, } 10 \%(\mathrm{v} / \mathrm{v}) \beta \text {-mercaptoethanol }\end{array}$ \\
\hline 50x TAE & $2 \mathrm{M}$ Tris, $0.05 \mathrm{M}$ EDTA pH $8,5.8 \%$ acetic acid \\
\hline $1 \times \mathrm{TE}$ & $10 \mathrm{mM}$ Tris $\mathrm{pH}$ 7.5, $1 \mathrm{mM}$ EDTA \\
\hline TFB I & $100 \mathrm{mM} \mathrm{RbCl}, 15 \%$ (v/v) glycerol, 0.5 mM LiCl (pH 5.8) \\
\hline TFB II & $\begin{array}{l}10 \mathrm{mM} \text { MOPS (pH 7), } 10 \mathrm{mM} \mathrm{RbCl,} 75 \mathrm{mM} \mathrm{CaCl} 2 \\
15 \% \text { (v/v) glycerol }\end{array}$ \\
\hline Transport buffer (TB) & $\begin{array}{l}20 \text { mM HEPES pH 7.3, } 110 \text { mM KOAc, } 2 \text { mM Mg(OAc) } \\
1 \text { mM EGTA }\end{array}$ \\
\hline Tris buffer & $\begin{array}{l}50 \mathrm{mM} \text { Tris- } \mathrm{HCl}(\mathrm{pH} 7.4), 200 \mathrm{mM} \mathrm{NaCl}, 1 \mathrm{mM} \mathrm{MgCl} \\
5 \% \text { glycerol, aprotinin, leupeptin, pepstatin, }(1 \mu \mathrm{g} / \mathrm{ml} \text { each })\end{array}$ \\
\hline Western blot buffer (WB) & $\begin{array}{l}192 \mathrm{mM} \text { glycine, } 25 \mathrm{mM} \text { Tris, } 0.05 \% \text { (v/v) SDS, } 20 \% \text { (v/v) } \\
\text { methanol }\end{array}$ \\
\hline
\end{tabular}




\begin{tabular}{l|l}
\hline Stock solutions & \\
\hline ampicillin & $100 \mathrm{mg} / \mathrm{ml}$ \\
aprotinin, 1000x & $1 \mathrm{mg} / \mathrm{ml}$ \\
$\mathrm{ATP}$ & in $100 \mathrm{mM} \mathrm{Mg}(\mathrm{OAc}), 2.20 \mathrm{mM}$ HEPES (pH7.4) \\
$\mathrm{CaCl}_{2}$ & $2.5 \mathrm{mM}$ \\
chloramphenicol & $30 \mathrm{mg} / \mathrm{ml}$ \\
digitonin & $10 \%(\mathrm{w} / \mathrm{v})$ in DMSO \\
dithiothreitol & $1 \mathrm{M}$ \\
Hoechst 33258 & $10 \mathrm{mg} / \mathrm{ml}$ \\
kanamycin & $50 \mathrm{mg} / \mathrm{ml}$ \\
leupeptin/pepstatin, 1000x & $1 \mathrm{mg} / \mathrm{ml}$ each, in DMSO \\
L-Glutamine, 100x & $200 \mathrm{mM}$ \\
phenylmethylsulfonyl fluoride & $100 \mathrm{mM}$ in 2-propanol \\
penicillin-streptomycin, 1000x & penicillin 10.000 U/ml streptomycin $10 \mathrm{mg} / \mathrm{ml}$
\end{tabular}

\begin{tabular}{l|l}
\hline Bacterial media & \\
\hline LB & $\begin{array}{l}1 \%(\mathrm{w} / \mathrm{v}) \text { bacto-tryptone, } 0.5 \%(\mathrm{w} / \mathrm{v}) \text { yeast extract, } 1 \%(\mathrm{w} / \mathrm{v}) \\
\mathrm{NaCl}(\mathrm{pH} 7.0)\end{array}$ \\
LB agar plates & LB supplemented with $1.5 \%(\mathrm{w} / \mathrm{v})$ bacto-agar \\
SOC & $\begin{array}{l}2 \%(\mathrm{w} / \mathrm{v}) \text { tryptone, } 5 \%(\mathrm{w} / \mathrm{v}) \text { yeast extract, } 50 \mathrm{mM} \mathrm{NaCl}, \\
2.5 \mathrm{mM} \mathrm{KCl}, 10 \mathrm{mM} \mathrm{MgCl}, 10 \mathrm{mM} \mathrm{MgSO} 4\end{array}$
\end{tabular}

\section{Cell culture media}

DMEM high (Gibco)

High Glucose (4500 mg/ml), 10\% (v/v) FCS, 2 mM

L-glutamine, $100 \mathrm{U} / \mathrm{ml}$ penicillin, $100 \mu \mathrm{g} / \mathrm{ml}$ streptomycin 


\subsubsection{Cell lines}

\begin{tabular}{l|l}
\hline Mammalian cells & \\
HeLa P4 & $\begin{array}{l}\text { adherent human cervix carcinoma cells that express CD4, } \\
\text { CXCR4, CCR5 receptors; contain the } \beta \text {-galactosidase-gene } \\
\text { under control of HIV-1 LTR (Charneau et al., 1994) }\end{array}$ \\
HeLa NFAT spinner & $\begin{array}{l}\text { HeLa cells that are stably transfected with a vector coding for } \\
\text { the fusion protein GFP-NFAT (nuclear factor of activated T- } \\
\text { cells; Hutten and Kehlenbach, 2006) }\end{array}$
\end{tabular}

\begin{tabular}{l|l}
\hline Bacteria & \\
\hline BL21-CodonPlus (DE3)-RIL & $\begin{array}{l}\text { F- ompT hsdS(rB- mB-) dem+ Tetr gal } \lambda(\mathrm{DE} 3) \text { endA Hte } \\
\text { [argU ileY leuW Camr] } \\
\text { hsdR17 recA1 end A1 gyrA96 thi-1relA1 }\end{array}$ \\
DH5 & $\begin{array}{l}\text { he } \\
\text { hel }\end{array}$
\end{tabular}




\subsubsection{Antibodies}

Primary antibodies

\begin{tabular}{l|l|l|l|l}
\hline Name & Species & Origin & Dilution & Application \\
\hline anti CRM1 & goat & R. Kehlenbach/S. Roloff & $1: 1000$ & WB, IF \\
anti HA & rabbit & Sigma Aldrich & $1: 500$ & WB, IF \\
anti His (penta) & mouse & Qiagen & $1: 1000$ & WB, IF \\
anti myc 9E10 & mouse & Serotec (WB)/Santa Cruz (IF) & $1: 5000 / 1: 200$ & WB, IF \\
anti Nup214 \#53 & rabbit & Hutten and Kehlenbach, 2006 & $1: 200$ & WB \\
anti Nup214 \#55 & rabbit & Hutten and Kehlenbach, 2006 & $1: 500$ & IF \\
anti Ran & mouse & BD Transduction Laboratories & $1: 5000$ & WB, IF
\end{tabular}

\section{Secondary antibodies}

\begin{tabular}{l|l|l|l|l}
\hline Name & Species & Origin & Dilution & Application \\
\hline anti goat Alexa Fluor 488 & donkey & Molecular probes & $1: 1000$ & IF \\
anti mouse Alexa Fluor 594 & donkey & Molecular probes & $1: 1000$ & IF \\
anti rabbit Alexa Fluor 594 & donkey & Molecular probes & $1: 1000$ & IF \\
HRP anti goat & donkey & Dianova & $1: 5000$ & WB \\
HRP anti mouse & donkey & Dianova & $1: 5000$ & WB \\
HRP anti rabbit & donkey & Dianova & $1: 5000$ & WB
\end{tabular}




\subsubsection{Oligonucleotides}

All oligonucleotides were obtained from Sigma Aldrich. For tracing back the cloning strategies, the names of the oligonucleotides contain additional information about used restriction enzymes. The numbers are also listed in section 2.1.10 (see "Generated plasmids"). The cloning strategies for CRM1-chimeras are depicted in detail in the appendix.

Oligonucleotides for cloning

\begin{tabular}{|c|c|c|}
\hline \multicolumn{2}{|c|}{ Number/Name } & \multirow{2}{*}{$\begin{array}{l}\text { Sequence } 5 \rightarrow 3 \\
\text { TTTGCTAGCATGCCAGCAATTATGACAATG }\end{array}$} \\
\hline G275 & CRMIhs39NheI_F & \\
\hline G276 & CRMIhsBamHI_R & TTTGGATCCCGATCACACATTTCTTCTGGAATC \\
\hline G277 & CRMIce248NheI_F & TTTGCTAGCATGGCTGTCTCAGCAATGGAA \\
\hline G278 & CRMI ce3487BamHI_R & TTTGGATCCCGACGCATATCCTCATCTTCCAC \\
\hline G516 & CRM1-HR13- BamHIR & TTTGGATCCGGCTGAATTACAAAGGGGTGTC \\
\hline G540 & CRM1ce-1697-KpnI_F & TTTGGTACCACTGGAAATTTGTGAAGACGG \\
\hline G541 & CRM1ce-1700-KpnI_R & TTTGGTACCGAAGGAATCTCGGGTACTG \\
\hline G542 & CRM1hs-1668-KpnI_F & TTTGGTACCACTGGAAATTTCTGAAGACT \\
\hline G548 & CRM1hs-1668_KpnI_R & TTTGGTACCTCAAAAAACGTGGGTATTGA \\
\hline G559 & CRM1-HR16_BamHIR & TTTGGATCCCGTGGGAAACAATGAGAATTGAC \\
\hline G560 & CRM1-HR18_BamHIR & TTTGGATCCCGGTTGTTAACTGGATTTCCCG \\
\hline G618 & CRM1ce1166-KpnI_R & TTTGGTACCGAATGGACATATACGGTAGAGC \\
\hline G619 & CRM1hs-1165-KpnI_F & TTTGGTACCTCTGGTACCTCTGCCTCT \\
\hline G622 & CRM1hs 1188_KpnI_R & TTTGGTACCAGAAAGCAACGGAGAGGC \\
\hline G623 & CRM1ce 1228-KpnI_F & TTTGGTACCCAGGTTCGTGAGCATCCA \\
\hline G785 & Nup214C EcoRI $1859 \mathrm{~F}$ & TTTGAATTCATAGTCTTTGGCCAGCAATCATCC \\
\hline G786 & Nup214C SalI 2090 R & TTTGTCGACGGCTTCGCCAGCCACCAAAAC \\
\hline G787 & Nup214C SalI 1974 R & TTTGTCGACGTGGAGCAGCACCGAAGCCA \\
\hline G788 & Nup214C EcoRI $1975 \mathrm{~F}$ & TTTGAATTCGTGTTTGGCAGCCCTCCTAC \\
\hline G789 & Nup214C EcoRI $1829 \mathrm{~F}$ & TTTGAATTCGCAGCAACCTCTGGGTTCAG \\
\hline G790 & Nup214C EcoRI $1945 \mathrm{~F}$ & TTTGAATTCGGCACTTTCAGCTCTGGAGGAGG \\
\hline G791 & Nup214C EcoRI 1916 F & $\begin{array}{l}\text { TTTGAATTCTCAAATACCTCTAACCTATTT } \\
\text { GGAAACAG }\end{array}$ \\
\hline G792 & Nup214 CSalI 2033 R & TTTGTCGACGTGTGGTGTTGCTGCTGCTCC \\
\hline G791 & Nup214C EcoRI $1916 \mathrm{~F}$ & $\begin{array}{l}\text { TTTGAATTCTCAAATACCTCTAACCTATTT } \\
\text { GGAAACAG }\end{array}$ \\
\hline
\end{tabular}




\begin{tabular}{|c|c|c|}
\hline \multicolumn{2}{|c|}{ Number/Name } & \multirow{2}{*}{$\begin{array}{l}\text { Sequence } 5 \rightarrow 3 \\
\text { TTTGTCGACGTGTGGTGTTGCTGCTGCTCC }\end{array}$} \\
\hline G792 & Nup214 CSalI 2033 R & \\
\hline G783 & Nup214C EcoRI $1859 \mathrm{~F}$ & TTTGAATTCCGTCTTTGGCCAGCAATCATCCTCTTCC \\
\hline G802 & Nup214 EcoRI 1975 F & TTTGAATTCCGTGTTTGGCAGCCCTCCTAC \\
\hline G803 & Nup214 EcoRI 1829 F & TTTGAATTCCGCAGCAACCTCTGGGTTCAG' \\
\hline G804 & Nup214 EcoRI 1945 F & TTTGAATTCCGGCACTTTCAGCTCTGGAGGAGG \\
\hline G805 & Nup214 EcoRI 1916 F & $\begin{array}{l}\text { TTTGAATTCCTCAAATACCTCTACСCTATTTG } \\
\text { GAAACAG }\end{array}$ \\
\hline G806 & Nup214 SalI 2033 R & TTTGTCGACTTATGTGGTGTTGCTGCTGCTCC \\
\hline G823 & cNLS KpnI F & CCGCGGCCCAAAGAAAAAGAGGAAAGTTGGGTAAG \\
\hline G824 & cNLS BamHI R & $\begin{array}{l}\text { GATCCTTACCCAACTTTCCTCTTTTTCTTTGGGCC } \\
\text { GCG GGTAC }\end{array}$ \\
\hline G831 & Nup214 2090 SalI R & TTTGTCGACGCTTCGCCAGCCACCAAAAG \\
\hline G832 & Nup214 1974 SalI R & TTTGTCGACTGGAGCAGCACCGAAGCCA \\
\hline G833 & Nup214 2033 SalI R & TTTGTCGACTGTGGTGTTGCTGCTGCTCC \\
\hline G841 & Nup214 1991 EcoRI F & TTTGAATTCTAGTGCCAGCATTCGGTTCAGC \\
\hline G848 & Nup214 1991 EcoRI F & TTT GAATTC C GTG CCA GCA TTC GGT TCA GC \\
\hline G849 & Nup214 a3 1916 EcoRI F & TTTGAATTCCAGCAATACCAGCAATCTGAGCG \\
\hline G850 & Nup214 a3 2033 SalI R & TTTGTCGACGGTGGTATTACTTGAGCTACCAC \\
\hline G851 & Nup214 1968 EcoRI F & TTTGAATTCTAGGTGGCTTCGGTGCTGCT \\
\hline G864 & Nup214 1990 SalI R & TTTGTCGACTTACCCTCCAAACCCAGGGGAT \\
\hline G865 & Nup214 a1 1968 EcoRI F & TTTGAATTCTAGGTGGTAGTGGTGCAGCAC \\
\hline G866 & Nup214 a1 1990 SalI R & TTTGTCGACTTAACCACCTGAACCCGGAGAAC \\
\hline G867 & Nup214 a1 2033 SalI R & TTTGTCGACTTAGGTGGTATTACTGCTGCTACCAAA \\
\hline G885 & Nup214a1, a2 1916F & TTTGAATTCCAGCAATACCAGCAACCTGTTTGGT \\
\hline G886 & Nup214 a1 2033R & TTTGTCGAC GGTGGTATTACTGCTGCTACCAAA \\
\hline G889 & Nup214 1968 EcoRI F & TTTGAATTCCGGTGGCTTCGGTGCTG \\
\hline G890 & Nup214 1968 a1 EcoR F & TTTGAATTCCGGTGGTAGTGGTGCAGCAC \\
\hline G891 & Nup214 1968 a2 EcoR F & TTTGAATTCCGGTGGCTTTGGTGCAGCAG \\
\hline G892 & Nup214 1990WT SalI R & TTTGTCGACCCCTCCAAACCCAGGGGAT \\
\hline G893 & Nup214 1990a1 SalI R & TTTGTCGACACCACCTGAACCCGGAGAAC \\
\hline G936 & NUP214 2034 EcoRIF & TTTGAATTCCTCCTTCGGCACGCTCGC \\
\hline
\end{tabular}


Oligonucleotides for mutagenesis

\section{Number/Name}

G651 CRM1 HS-loop del_F KpnI

G652 CRM1 HS-loop del_R KpnI
Sequence $5 \rightarrow 3^{\prime}$

TGAACTCTATAGAGAGGGTACC $(\Delta)$ CCCAGG AGACAGCTAT

ATAGCTGTCTCCTGGGGGTACC ( $\Delta)$ CTCTC

TATAGAGTTCA

Oligonucleotides for sequencing

Number/Name

G379 CRM1-ce-501F

G380 CRM1-ce-400R

G381 CRM1-ce-1501F

G382 CRM1-ce-1001F

G383 CRM1-ce-1501F

G384 CRM1-ce-2001F

G385 CRM1-ce-2501F

G386 CRM1-ce-3001F

G387 CRM1-hs-39F

G388 CRM1-hs-200R

G389 CRM1-hs-501F

G390 CRM1-hs-1001F

G391 CRM1-hs-1501F

G392 CRM1-hs-2001F

G393 CRM1-hs-2495F

G394 CRM1-hs-3001F

G525 T7-Promotor

G545 pmCherry_seq_f

G546 pmCherry_seq_r
Sequence $5 \rightarrow 3$

TCATCCAACACAAATGGAAGT

CGACATGAGGATTTGGTTAGC

CTACCGTGAATACCTCTCGCA

AACTTCCTTTCGCTTGAAGTG

CTACCGTGAATACCTCTCGCA

AGACTCATGAAGGTGTACAGG

AATCCATTGGTAAAGACGATG

TGACAAGATCGCTCAACCATT

ATGCCAGCAATTATGACAATG

CTTTAAATGTGTCAGTACTTC

GGAGCAAGTAGGACCAGCGAA

TCTCAGTTTGTTTCTCTGCAC

AGTGGTCATGGAAAAATTTGA

GAAAAGTACATGTTACTCCCT

ACCTCAAATATTTGAATGCTG

ACCTACAAGATGCTCAAGTAA

TAATACGACTCACTATAGGG

CGCTGAGGTCAAGACCACC

CGAAGGGCAGGGGGCCACC 


\subsubsection{Vectors and plasmids}

\section{Available vectors}

\begin{tabular}{ll|l|l|l|l}
\hline \multicolumn{2}{l|}{ Number/Name } & Tag & Resistance & Application & Source \\
\hline 4 & pET-28a & His & kanamycin & protein expression & Novagen \\
$47 \quad$ pGex-6P-1-MCS & GST & ampicillin & protein expression & $\begin{array}{l}\text { Amersham/ } \\
\text { S. Hutten } \\
\text { Invitrogen/ }\end{array}$ \\
$52 \quad$ pcDNA3.1(+)-HA & HA & ampicillin & transfection & $\begin{array}{l}\text { S. Wälde } \\
\text { Clontech }\end{array}$ \\
& pmRFP-C1 & RFP & kanamycin & transfection &
\end{tabular}

\section{Available plasmids}

\begin{tabular}{|c|c|c|c|c|c|}
\hline \multicolumn{2}{|c|}{ Number/Name } & \multirow{2}{*}{$\begin{array}{l}\text { Tag } \\
\text { GST }\end{array}$} & \multirow{2}{*}{$\begin{array}{l}\text { Vector } \\
\text { pGEX-KG }\end{array}$} & \multirow{2}{*}{$\begin{array}{l}\text { Application } \\
\text { protein expression }\end{array}$} & \multirow[t]{2}{*}{ Source } \\
\hline 5 & GST-Ran & & & & \\
\hline 48 & His-Nup214 & His & pTRC & protein expression & R. Kehlenbach \\
\hline 47 & Nup214 & & pBluescript & cloning/mutagenesis & G. Grosfeld \\
\hline 487 & pGFP-NES(REV) & GFP & pEGFP-C3 & transfection & S. Hutten \\
\hline 531 & CRM1 C. elegans & HA & pcDNA3.1 HA & transfection & C. Spillner \\
\hline 532 & CRM1 H. sapiens & HA & pcDNA3.1 HA & transfection & C. Spillner \\
\hline 627 & CRM1 C.e. C538S & HA & pcDNA3.1 HA & transfection & $\begin{array}{l}\text { S. Roloff } \\
\text { (Dipl. thesis) }\end{array}$ \\
\hline 628 & CRM1 H.s. C528S & HA & pcDNA3.1 HA & transfection & $\begin{array}{l}\text { S. Roloff } \\
\text { (Dipl. thesis) }\end{array}$ \\
\hline 661 & Myc-Nup214 1859-2090 & Myc & pEF-Myc MCS & transfection & $\begin{array}{l}\text { S. Roloff } \\
\text { (Dipl. thesis) }\end{array}$ \\
\hline 856 & His-SPN1 & His & pET30-b & protein expression & I. Waldmann \\
\hline 857 & GFP-SPN1 & GFP & pEGFP-C1 & transfection & $\begin{array}{l}\text { Waldmann et } \\
\text { al., } 2012\end{array}$ \\
\hline
\end{tabular}




\section{Purchased plasmids}

\begin{tabular}{ll|l|l|l|l}
\hline \multicolumn{2}{l|}{ Number/Name } & Tag & Vector & Application & Source \\
\hline 949 & Nup214 aa1916-2033 a1 & no & pMA & cloning & life technologies \\
$950 \quad$ Nup214 aa1916-2033 a2 & no & pMA & cloning & life technologies \\
951 & Nup214 aa1916-2033 a3 & no & pMA & cloning & life technologies \\
1025 & Nup214 aa1859-2090 a1 & no & pMA-T & cloning & life technologies \\
1036 & Nup214 aa1859-2090 a3 & no & pMA-T & cloning & life technologies
\end{tabular}

\section{Generated plasmids}

\begin{tabular}{|c|c|c|c|c|c|}
\hline \multicolumn{2}{|c|}{ Number/Name } & \multirow{2}{*}{$\begin{array}{l}\text { Tag } \\
\text { HA }\end{array}$} & \multirow{2}{*}{$\begin{array}{l}\text { Vector } \\
\text { pcDNA3.1 HA }\end{array}$} & \multirow{2}{*}{$\begin{array}{l}\text { Application } \\
\text { transfection }\end{array}$} & \multirow{2}{*}{$\begin{array}{l}\text { Cloning } \\
\text { G275/G516 }\end{array}$} \\
\hline 694 & CRM1 aa $1-709$ C528S & & & & \\
\hline 713 & CRM1 aa $1-861 \mathrm{C} 528 \mathrm{~S}$ & HA & pcDNA3.1 HA & transfection & G275/G559 \\
\hline 714 & CRM1 aa $1-970$ C528S & HA & pcDNA3.1 HA & transfection & G275/G560 \\
\hline 721 & CRM1 H.s./C.e. I C528S & HA & pcDNA3.1 HA & transfection & $\begin{array}{l}\text { G275/G548/G540/ } \\
\text { G529 }\end{array}$ \\
\hline 722 & CRM1 C.e./H.s. I C538S & HA & pcDNA3.1 HA & transfection & $\begin{array}{l}\text { G277/G541/G542/ } \\
\text { G276 }\end{array}$ \\
\hline 743 & CRM1 C.e./H.s. II C528S & HA & pcDNA3.1 HA & transfection & $\begin{array}{l}\text { G277/G618/G619/ } \\
\text { G276 }\end{array}$ \\
\hline 744 & CRM1 H.s./C.e. II C538S & HA & pcDNA3.1 HA & transfection & $\begin{array}{l}\text { G275/G622/G623/ } \\
\text { G529 }\end{array}$ \\
\hline 749 & CRM1 H.s. $\Delta$ loop C528S & HA & pcDNA3.1 HA & transfection & $\begin{array}{l}\text { Mutagenesis; G651/ } \\
\text { G652 }\end{array}$ \\
\hline 943 & His-Nup214 aa1859-2090 & His & pET28-a & protein expr. & G785/G786 \\
\hline 944 & His-Nup214 aa1859-1974 & His & pET28-a & protein expr. & G785/G787 \\
\hline 945 & His-Nup214 aa1975-2090 & His & pET28-a & protein expr. & G788/G786 \\
\hline 946 & His-Nup214 aa1829-1974 & His & pET28-a & protein expr. & G789/G787 \\
\hline 947 & His-Nup214 aa1945-2090 & His & pET28-a & protein expr. & G790/786 \\
\hline 948 & His Nup214 aa1916-2033 & His & pET28-a & protein expr. & G791/G792 \\
\hline 987 & RFP-cNLS & RFP & pmRFP-C1 & transfection & $\begin{array}{l}\text { Oligo annealing; } \\
\text { G823/G824 }\end{array}$ \\
\hline 988 & RFP-Nup214 1859-2090 cNLS & RFP & pmRFP-C1 & transfection & G783/G831 \\
\hline 989 & RFP-Nup214 1859-1974 cNLS & RFP & pmRFP-C1 & transfection & G783/G832 \\
\hline 990 & RFP-Nup214 1975-2090 cNLS & RFP & pmRFP-C1 & transfection & G802/G831 \\
\hline 991 & RFP-Nup214 1829-1974 cNLS & RFP & pmRFP-C1 & transfection & G803/G832 \\
\hline
\end{tabular}


2 Material and methods

\begin{tabular}{|c|c|c|c|c|c|}
\hline \multicolumn{2}{|c|}{ Number/Name } & \multirow{2}{*}{$\begin{array}{l}\text { Tag } \\
\text { RFP }\end{array}$} & \multirow{2}{*}{$\begin{array}{l}\text { Resistance } \\
\text { pmRFP-C1 }\end{array}$} & \multirow{2}{*}{$\begin{array}{l}\text { Application } \\
\text { transfection }\end{array}$} & \multirow{2}{*}{\begin{tabular}{|l} 
Cloning \\
G804/G831
\end{tabular}} \\
\hline 992 & RFP-Nup214 1945-2090 cNLS & & & & \\
\hline 993 & RFP-Nup214 1916-2033 cNLS & RFP & pmRFP-C1 & transfection & G805/G833 \\
\hline 994 & $\begin{array}{l}\text { RFP-Nup214 1916-2033- } \\
\text { cNLS a1 }\end{array}$ & RFP & pmRFP-C1 & transfection & G885/G886 \\
\hline 995 & $\begin{array}{l}\text { RFP-Nup214 1916-2033 } \\
\text { cNLS a2 }\end{array}$ & RFP & pmRFP-C1 & transfection & G885/G886 \\
\hline 996 & $\begin{array}{l}\text { RFP-Nup214 1916-2033 } \\
\text { cNLS a3 }\end{array}$ & RFP & pmRFP-C1 & transfection & G849/G850 \\
\hline 997 & His-Nup214 1916-2033 a1 & His & pET28-a & protein expr. & $\begin{array}{l}\text { from } 949 \text { via EcoRI/ } \\
\text { SalI }\end{array}$ \\
\hline 998 & His-Nup2141916-2033 a2 & His & pET28-a & protein expr. & $\begin{array}{l}\text { from } 950 \text { via EcoRI/ } \\
\text { SalI }\end{array}$ \\
\hline 999 & His-Nup214 1916-2033 a3 & His & pET28-a & protein expr. & $\begin{array}{l}\text { from } 951 \text { via EcoRI/ } \\
\text { SalI }\end{array}$ \\
\hline 1000 & GST-Nup214 1991-2033 & GST & pGEX-6P-1 & protein expr. & G841/G806 \\
\hline 1007 & GST-Nup214 1968-1990 WT & GST & pGEX-6P-1 & protein expr. & G851/G864 \\
\hline 1008 & GST-Nup214 1968-1990 a1 & GST & pGEX-6P-1 & protein expr. & G865/G866 \\
\hline 1009 & GST-Nup214 1968-2033 WT & GST & pGEX-6P-1 & protein expr. & G851/G806 \\
\hline 1010 & GST-Nup214 1968-2033 a1 & GST & pGEX-6P-1 & protein expr. & G865/G867 \\
\hline 1011 & RFP-Nup214 1968-2033 WT & RFP & pmRFP-C1 & transfection & G889/G833 \\
\hline 1012 & RFP-Nup214 1968-2033 a1 & RFP & pmRFP-C1 & transfection & G890/G886 \\
\hline 1030 & $\begin{array}{l}\text { RFPNup214 1859-2090- } \\
\text { cNLS a1 }\end{array}$ & RFP & pmRFP-C1 & transfection & $\begin{array}{l}\text { from } 1025 \text { via EcoRI/ } \\
\text { SalI }\end{array}$ \\
\hline 1031 & $\begin{array}{l}\text { RFP-Nup214 1916-1990- } \\
\text { cNLS WT }\end{array}$ & RFP & pmRFP-C1 & transfection & G805/G892 \\
\hline 1032 & $\begin{array}{l}\text { RFP-Nup214 1968-1990- } \\
\text { cNLS a1 }\end{array}$ & RFP & pmRFP-C1 & transfection & G890/G893 \\
\hline 1033 & $\begin{array}{l}\text { RFP-Nup214 1968-1990- } \\
\text { cNLS WT }\end{array}$ & RFP & pmRFP-C1 & transfection & G889/G892 \\
\hline 1034 & RFP-Nup214 1991-2033cNLS & RFP & pmRFP-C1 & transfection & G848/G833 \\
\hline 1035 & RFP-Nup214 1991-2090cNLS & RFP & pmRFP-C1 & transfection & G848/G831 \\
\hline 1038 & RFP-Nup214 1916-1990cNLS & RFP & pmRFP-C1 & transfection & G805/G892 \\
\hline 1039 & RFP-Nup214 2034-2090cNLS & RFP & pmRFP-C1 & transfection & G936/G831 \\
\hline
\end{tabular}




\subsubsection{Proteins}

The following proteins are available from common lab-stocks.

\begin{tabular}{l|l|l}
\hline Name & Species & Reference \\
\hline His-CRM1 & H. sapiens & Guan et al., 2000 \\
Ran WT & H. sapiens & Melchior et al., 1995 \\
His-SPN1 & H. sapiens & Waldmann et al., 2011
\end{tabular}

The proteins listed below were purifed in this work (see section 2.2.2).

\begin{tabular}{l|l|l}
\hline Name & Species & purification method \\
His-Nup214 1859-2090 & H. sapiens & Ni-NTA agarose \\
His-Nup214 1859-1974 & H. sapiens & Ni-NTA agarose \\
His-Nup214 1916-2033 WT/mutants & H. sapiens & Ni-NTA agarose, S75 \\
GST-Nup214 1968-1990 WT/a1 mutant & H. sapiens & glutathione sepharose, \\
& Standard purification \\
GST-Nup214 1968-2033 WT/a1 mutant & H. sapiens \\
GST-Nup214 1991-2033 & H. sapiens
\end{tabular}




\subsection{Biochemical methods}

\subsubsection{Separation and detection of proteins}

\section{$\underline{\text { Separation of proteins with SDS polyacrylamide gel electrophoresis }}$}

SDS (sodium dodecyl sulfates) polyacrylamide gel electrophoresis (SDS-PAGE) is a method for efficient separation of proteins dependent on their molecular weight. It was firstly described by Laemmli et al., 1970. Based on that, an SDS-containing, discontinuos Tris-HCl/Tris-glycine buffer system was used in this work. According to the size of the proteins, 6-12\% gels, consisting of a stacking and a resolving gel, were prepared with the SE260 Hoefer system. Before loading to the gel pockets, the protein samples were heated in SDS-PAGE loading buffer (1x final concentration) for 5 minutes at $95^{\circ} \mathrm{C}$. The gels were run in $1 \mathrm{x}$ Laemmli buffer with $25 \mathrm{~mA}$ per gel at maximum voltage.

SDS-PAGE loading buffer (4x)

10x Laemmli running buffer
$4 \%$ (w/v) SDS, $125 \mathrm{mM}$ Tris pH 6.8, 10\% (v/v) glycerol, $0.02 \%(\mathrm{w} / \mathrm{v})$ bromphenol blue, $10 \%(\mathrm{v} / \mathrm{v}) \beta$-mercaptoethanol $250 \mathrm{mM}$ Tris, $1.92 \mathrm{M}$ glycine, $0.1 \%$ (w/v) SDS

\section{Detection of proteins with coomassie and colloidal coomassie staining}

After gel electrophoresis, the gels were incubated with coomassie fixing solution for 30 minutes. The fixing solution was removed and coomassie dye was added for maximal 5 minutes. In order to remove excess of the dye the gels were washed with water for several times. With this staining method, protein amounts as little as $100 \mathrm{ng}$ can be detected.

coomassie fixing solution

coomassie dye
$45 \%(\mathrm{v} / \mathrm{v})$ methanol, $10 \%(\mathrm{v} / \mathrm{v})$ acetic acid

$10 \%(\mathrm{v} / \mathrm{v})$ acetic acid, $0.025 \%(\mathrm{w} / \mathrm{v})$ brilliant blue G-250

For detection of smaller amounts of proteins ( $\geq 30 \mathrm{ng}$ ) the gels were incubated in colloidal coomassie fixing solution for 60 minutes. After washing with water for 10 minutes, the gels were incubated with colloidal coomassie dye over night. The dye has to be prepared at least one day before without methanol. Prior staining, methanol has to be added freshly. 
Colloidal coomassie fixing solution

Colloidal coomassie dye
$40 \%(\mathrm{v} / \mathrm{v})$ ethanol, $10 \%(\mathrm{v} / \mathrm{v})$ acetic acid

$0.1 \%(\mathrm{v} / \mathrm{v})$ brilliant blue G-250, $2 \%$ (w/v) orthophosphoric acid, $10 \%(\mathrm{w} / \mathrm{v})$ ammonium sulphate, $20 \%(\mathrm{v} / \mathrm{v})$ methanol

\section{Transfer of proteins to nitrocellulose (Western blot) followed by immunological detection}

Dependent on the molecular weight of the analyzed proteins, either the semi-dry or the wet blot technique was applied. The SDS gel was placed directly onto a wet nitrocellulose membrane (equilibrated with Western blot buffer). For stabilization, two whatman papers (also soaked with Western blot buffer) were packed on each side. Depending on the method, this package was either clamped into a wet-blot chamber, filled with buffer, or laid into a semi-dry chamber. The wet blot chamber was run at $400 \mathrm{~mA}$ for 1.5-2 hours. The semi-dry chamber was run at $200 \mathrm{~mA}$ per gel for 1.5 hours.

The transfer efficiency was analyzed by reversible staining of the nitrocellulose membrane with Ponceau S solution. The membrane was incubated with the solution for 5 minutes, followed by washing with $1 \%$ acetic acid, until red protein bands become visible.

For immunological detection of single proteins, the nitrocellulose was first blocked with $3 \%$ milk in PBS-Tween buffer for 30 minutes. The primary antibodies was diluted in the same buffer and added to the membrane. After incubation over night at $4{ }^{\circ} \mathrm{C}$, the membrane was washed three times with PBSTween buffer without milk. The secondary antibody, coupled with horseradish peroxidase and directed against the species of the primary antibody, was diluted in PBS-Tween buffer with $3 \%$ milk and the membrane was incubated for two hours at room temperature. The membrane was washed for three times, followed by addition of chemiluminescent reagents (Millipore). Depending on the expected signal, the membrane was incubated for maximal five minutes. For detection of the signals, autoradiography films were used and developed with an automatic developer machine. Alternatively, the signals were directly detected with a digital documentation system (LAS-3000, Fuji).

Western blot buffer

PBS Tween
$192 \mathrm{mM}$ glycine, $25 \mathrm{mM}$ Tris, $0.05 \%$ (v/v) SDS, $20 \%$ (v/v) methanol

$140 \mathrm{mM} \mathrm{NaCl}, 2.5 \mathrm{mM} \mathrm{KCl}, 10 \mathrm{mM} \mathrm{Na}_{2} \mathrm{HPO}_{4}, \mathrm{KH}_{2} \mathrm{PO}_{4}(\mathrm{pH} 7.5)$, $0.1 \%(\mathrm{v} / \mathrm{v})$ Tween 20 


\subsubsection{Protein preparation}

\section{Purification of His-Nup214-fragments}

The corresponding vector constructs were transformed into BL21-CodonPlus (DE3)-RIL bacteria. The transformed bacteria were plated on LB-agar plates containing kanamycin and chloramphenicol, grown for one day and used for inoculation of $21 \mathrm{LB}$ medium. The cultures were grown to an $\mathrm{OD}_{600}$ 0.5-0.8 and the protein expression was induced with $0.1 \mathrm{mM}$ IPTG. The bacteria were kept over night at $18{ }^{\circ} \mathrm{C}$. The cell suspension was centrifuged at $4000 \mathrm{rpm}$ for 10 minutes, and the pellet was resuspended in Nup214-buffer containing $1 \%$ Triton X-100 and $20 \mathrm{mM}$ imidazole. The cells were lysed and the lysate was cleared by centrifugation at $100000 \mathrm{x}$ g for 30 minutes. Afterwards, Ni-NTA agarose was added to the lysate ( $300 \mu 1$ per $1 \mathrm{~L}$ culture) and the whole suspension was rotated for 1.5 hours at $4{ }^{\circ} \mathrm{C}$. The Ni-NTA agarose was washed three times for at least 10 minutes. Bound proteins were eluted with $500 \mathrm{mM}$ imidazole and dialyzed against Nup214 buffer with $2 \mathrm{mM}$ DTT instead of $\beta$-mercaptoethanol and without imidazole.

Nup214 buffer

$50 \mathrm{mM}$ Tris- $\mathrm{HCl}$ (pH 6.8), $300 \mathrm{mM} \mathrm{NaCl}, 10 \%$ glycerol, $4 \mathrm{mM} \beta$-mercaptoethanol, $1 \mathrm{mM} \mathrm{MgCl}$, aprotinin, leupeptin, pepstatin, $(1 \mu \mathrm{g} / \mathrm{ml}$ each),

$0.1 \mathrm{mM}$ PMSF

\section{Purification of GST-Nup214-fragments}

BL21-CodonPlus (DE3)-RIL bacteria were transformed with plasmid DNA, plated on LB agar plates containing ampicillin and kanamycin and grown for one day and used for inoculation of $21 \mathrm{LB}$ medium. Cultures were grown to an $\mathrm{OD}_{600} 0.4$ and induced with $0.1 \mathrm{mM}$ IPTG. The temperature was shifted to $16{ }^{\circ} \mathrm{C}$ and the bacteria were kept over night. The bacteria suspension was centrifuged at 400 rpm for 10 minutes and the pellet was resuspended in GST-buffer containing $0.5 \%$ Triton-X 100 . Afterwards, the bacteria were lysed and the lysate was cleared by centrifugation at $100000 \mathrm{x} g$ for 30 minutes. Before adding the glutathione sepharose, the supernatant was diluted 1:4 in GST buffer without Triton-X 100. The samples were rotated for 1.5 hours at $4{ }^{\circ} \mathrm{C}$, washed three times for at least 10 minutes, and bound proteins were eluted with $15 \mathrm{mM}$ reduced glutathione $\mathrm{pH}$ 8.0. The proteins were dialyzed against transport buffer.

GST buffer

$50 \mathrm{mM}$ Tris- $\mathrm{HCl}$ (pH 6.8), $300 \mathrm{mM} \mathrm{NaCl}, 0.25 \mathrm{mM}$ EDTA, $1 \mathrm{mM} \mathrm{MgCl}_{2}$, aprotinin, leupeptin, pepstatin, $(1 \mu \mathrm{g} / \mathrm{ml}$ each $), 0.1 \mathrm{mM}$ PMSF 


\subsubsection{In vitro binding assays with GST-tagged proteins}

All binding studies were performed in Tris buffer. Per sample, $5 \mu \mathrm{g}$ of GST-tagged protein were immobilized for one hour on $20 \mu \mathrm{l}$ glutathione sepharose beads (equilibrated before in Tris buffer). Unbound proteins were removed by washing the beads for three times, and unspecific binding sites were blocked by adding Tris buffer containing $20 \mathrm{mg} / \mathrm{ml}$ BSA for 20 minutes. After that, the proteins to be investigated were added and the whole suspension was incubated for an hour. The sepharose beads were washed three times and then the supernatant was completely taken off by using a gel loading tip which was gently pressed before with a forceps. Finally, $50 \mu \mathrm{l}$ of $2 \mathrm{x}$ sample buffer was added to the beads and the samples were heated for 5 minutes at $95{ }^{\circ} \mathrm{C}$.

Tris buffer

$50 \mathrm{mM}$ Tris- $\mathrm{HCl}$ (pH 7.4), $200 \mathrm{mM} \mathrm{NaCl}, 1 \mathrm{mM} \mathrm{MgCl} 2,5 \%$ glycerol, aprotinin, leupeptin, pepstatin, $(1 \mu \mathrm{g} / \mathrm{ml}$ each)

Loading of Ran with GDP/GTP (Kehlenbach et al., 1999):

Per sample, $10 \mu \mathrm{g}$ Ran were incubated for 30 minutes at room temperature together with $4.5 \mathrm{mM}$ EDTA and $10 \mathrm{mM} \mathrm{GDP/GTP}$ in Tris-buffer ( $150 \mu$ final volume). Afterwards, $30 \mathrm{mM} \mathrm{MgCl}_{2}$ was added, followed by incubation for 15 minutes on ice.

\section{Pulldown assays with GST-Nup214-fragments:}

Immobilized GST-Nup214-fragments (400 nM) were incubated with $960 \mathrm{nM}$ RanGDP/GTP, $255 \mathrm{nM}$ His-SPN1 and 108 nM His-CRM1 in a final volume of $400 \mu 1$. Bound proteins were analyzed by SDSPAGE and coomassie staining as described above.

\section{Pulldown assays with GST-Ran:}

Before immobilizing GST-Ran $(500 \mathrm{nM})$ to glutathione sepharose, it was loaded with either GDP or GTP. 108 nM His-CRM1, 160 nM His-Nup214-fragments and 255 nM His-SPN1 (or 2500 nM NES peptide) were added. For detection of His-tagged proteins, a Western blot using a His-antibody was performed. Detection of small His-Nup214-fragments bound to CRM1 is also possible by colloidal coomassie staining. 


\subsubsection{Affinity purification of CRM1 antibodies}

A goat (bread at the Institut für Tierzucht und Haustiergenetik, Göttingen) was immunized with the Cterminal CRM1 peptide GIFNPHEIPEEMCD coupled to KLH (Keyhole Limpet Hemacyanin). The first injection (injections were done by the veterinarian at intervals of four weeks) was prepared with Titermax ${ }^{\circledR}$ Gold, the following two injections were prepared with incomplete Freundsches Adjuvanz. After the second injection, a small volume of blood was withdrawed (3 weeks after) to determine the immunization progress. Final blood was withdrawed three weeks after the third injection. Blood was collected in 11 centrifuge falcons, stirred with a glass rod for 1 minute and stored at $4{ }^{\circ} \mathrm{C}$ over night. The next day, the blood was stirred again and centrifuged for 30 minutes at $4000 \mathrm{rpm}$ and $4{ }^{\circ} \mathrm{C}$. The supernatant (final serum containing the antibodies) was collected and frozen in aliquots. For affinity purification of the antibodies, $0.4 \mathrm{~g}$ of Cyanogen bromide-activated Sepharose 4B (CnBr beads) were swollen in $3 \mathrm{ml} \mathrm{HCl}(1 \mathrm{mM})$ for 10 minutes and equilibrated with $0.2 \mathrm{M}$ carbonate buffer $\mathrm{pH}$ 8.9. 1 mg of the CRM1 peptide was dissolved in carbonate buffer and added to the $\mathrm{CnBr}$ beads. The beads were rotated for 1 hour at room temperature and afterwards over night at $4{ }^{\circ} \mathrm{C}$. The $\mathrm{CnBr}$ beads were washed twice with carbonate buffer, and binding sites were saturated with $100 \mathrm{mM}$ ethanolamine for 1 hour at room temperature. After washing the beads three times with carbonate buffer, they were equilibrated with $500 \mathrm{mM} \mathrm{NaCl}$ in PBS. $15 \mathrm{ml}$ of goat serum were incubated with $2 \mathrm{ml}$ of the $\mathrm{CnBr}$ bead solution over night at $4{ }^{\circ} \mathrm{C}$ (in a $50 \mathrm{ml}$ falcon, completely filled up with $500 \mathrm{mM} \mathrm{NaCl}$ in PBS). The beads were washed twice with $\mathrm{NaCl} / \mathrm{PBS}$ and applied to a polystyrene column and washed until no proteins could be detected anymore with protein assay reagent (BIO-RAD). The antibodies were eluted with $500 \mathrm{mM} \mathrm{NaCl}$ in $0.2 \mathrm{M}$ acetic acid $\mathrm{pH} 2.7$ in $0.5 \mathrm{ml}$ fractions. The fractions were neutralized immediately with $100 \mu \mathrm{l}$ of $1 \mathrm{M}$ Tris and analyzed with Ponceau-S on nitrocellulose membrane. The pooled fractions were concentrated with centrifugal filter units. Finally, $2.5 \mathrm{mg}$ CRM1 antibody could be obtained from $15 \mathrm{ml}$ serum.

\section{$\underline{\text { Required buffers and solutions for antibody purification }}$}

$0.2 \mathrm{M}$ carbonate buffer $\mathrm{pH} 8.9$

$100 \mathrm{mM}$ ethanolamine

$500 \mathrm{mM}$ NaCL in PBS

$500 \mathrm{mM} \mathrm{NaCl}$ in $0.2 \mathrm{M}$ acetic acid $\mathrm{pH} 2.7$

$1 \mathrm{M}$ Tris 
For characterization of the CRM1-antibody, HeLa cells and recombinant His-CRM1 were analyzed. Endogenous CRM1 could be detected in immunofluorescence as well as in Western blot (Fig 7A and B). The antibody recognized $1 \mathrm{ng}$ of His-CRM1. The side bands appearing in the cell lysate sample could derive from degradation of CRM1.

(A)
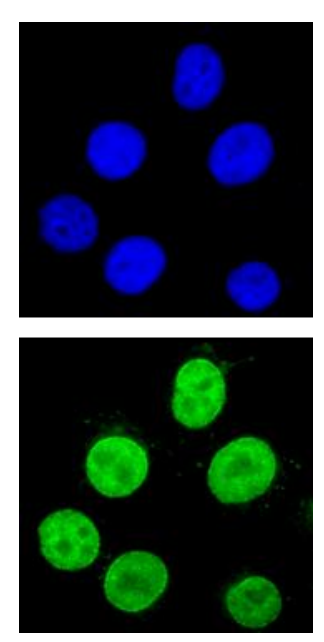

DNA

endogenous

CRM1
(B)

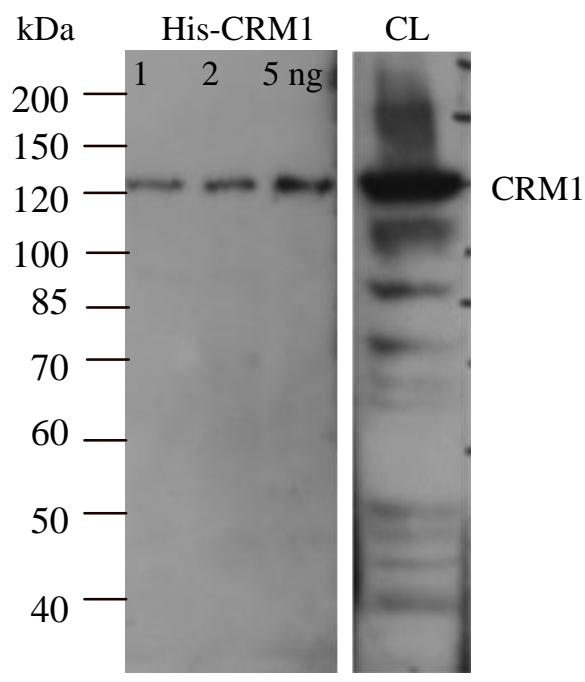

Fig. 7 Characterization of purified CRM1 antibody. (A) HeLa cells were fixed, stained for endogenous CRM1 and analyzed by immunofluorescence microscopy. The antibody was diluted 1:5000, corresponding to $40 \mathrm{ng}$ of antibody per slide. (B) 1, 2 or $5 \mathrm{ng}$ of recombinant His-CRM1 or lysate from HeLa cells (50000 cells; CL) was analyzed by Western blot (antibody dilution 1:5000; film exposure time 5 seconds). 


\subsection{Cell biology methods}

\subsubsection{Cell culture}

HeLa P4 cells were grown at $37{ }^{\circ} \mathrm{C}$ and $5 \% \mathrm{CO}_{2}$ on $10 \mathrm{~cm}$ diameter plates in Dulbecco's modified Eagle Medium (DMEM) containing 10\% fetal calf serum (FCS), $100 \mathrm{U} / \mathrm{ml}$ penicillin, $100 \mu \mathrm{g} / \mathrm{ml}$ streptomycin and $2 \mathrm{mM}$ L-glutamine. For transferring to other plates or to 24-wells containing glass cover slips, the cells were washed with PBS, detached from the plate with trypsin at $37{ }^{\circ} \mathrm{C}$ and diluted in fresh medium.

To improve adherence of the cells, glass coverslips were coated with poly L-lysine. After washing the coverslips in isopropanol, they were dried and placed on parafilm. $0.01 \%$ (v/v) poly-L-lysine (in sterile water) was dropped onto the coverslips. After 20 minutes, they were washed with sterile water, dried and exposed to UV light for 3 minutes.

\subsubsection{Transfection of DNA in mammalian cells}

Transfections were performed with the calcium phosphate method (Ausubel et al., 1994). HeLa P4 cells were grown on cover slips in 24 -well plates in $500 \mu 1$ medium until they reached $40 \%$ confluence. The amount of plasmid DNA per well did not exceed $1 \mu \mathrm{g}$, in the case of GFP-NC2 $\beta$, not more than $0.1 \mu \mathrm{g}$ was used. $20 \mu \mathrm{CaCl}_{2}(250 \mu \mathrm{M})$ was added and the tube was gently vortexed for 5 seconds. $20 \mu \mathrm{l}$ of $2 \mathrm{x}$ HEPES buffer was added, the mixture was vortexed for 10 seconds at the highest level, incubated for 20-30 minutes at room temperature and the solution was added to the cells. After transfection, cells were grown for 20-24 hours (Nup214-fragments) or for 48 hours (HA-CRM1 constructs). 


\subsubsection{In vitro transport assays}

$\underline{\text { Transport assay with GFP-NFAT cells (Kehlenbach et al., 1998) }}$

GFP-NFAT cells were grown to $50 \%$ confluence in a $10 \mathrm{~cm}$ diameter plate. The expression of GFPNFAT was induced with $1 \mu \mathrm{M}$ trichostatin A over night. Nuclear import of GFP-NFAT was induced by the addition of $1 \mu \mathrm{M}$ ionomycin for 20 minutes. Afterwards the cells were detached from the plate and resuspended in transport buffer (TB) containing 10\% FCS. From now on all steps were performed on ice. The cell suspension was centrifuged at $1000 \mathrm{rpm}$ for 5 minutes and TB without FCS was added to the pellet. The plasma membrane was permeabilized by adding 2-3 $\mu 1$ of $1 \%$ digitonin (per $1 \times 10^{6}$ cells) for maximal 5 minutes. Afterwards, the cells were washed twice with TB. Permeabilization was checked with trypane blue. The cells were resuspended to $1-2 \times 10^{7}$ cells $/ \mathrm{ml}$. Per sample reaction, 200.000 cells were incubated with transport factors in a final volume of $40 \mu \mathrm{l}$ at $30{ }^{\circ} \mathrm{C}$ or $4{ }^{\circ} \mathrm{C}$. The reactions were stopped by addition of ice-cold TB. Residual nuclear fluorescence of GFP-NFAT was analyzed by flow cytometry (FACS Canto $\left.{ }^{\mathrm{TM}} \mathrm{II}\right)$.

\section{$\underline{\text { Transport assay with adherent HeLa cells }}$}

HeLa P4 cells were grown on a coverslip to $80 \%$ confluence. All steps before incubation with transport factors were performed on ice. The cells were washed twice with TB and permeabilized with $0.05 \%$ digitonin. After 5 minutes, the cells were washed again, and permeabilization was checked with trypane blue. The reactions were assembled separately in tubes (50 $\mu$ l final volume) as indicated in the table below and were then added to the cells. After incubation at $30{ }^{\circ} \mathrm{C}$ or $4{ }^{\circ} \mathrm{C}$, the cells were washed with TB and fixed. The proteins to be investigated were detected by indirect immunofluorescence.

Transport buffer (TB)

20 mM HEPES pH 7.3, 110 mM KOAc, 2 mM Mg(OAc)

1 mM EGTA

\begin{tabular}{l|l}
\hline Reaction components & Concentration/amount per sample \\
\hline Cytosol (HeLa S4; Kehlenbach et al., 1998) & $5 \mu \mathrm{l}$ \\
Ran WT & $1.92 \mu \mathrm{M}$ \\
WGA & $0.04 \mathrm{mg} / \mathrm{ml}$ \\
ATP-regenerating system & $1 \mu \mathrm{l}$ \\
Nup214 aa 1916-1933 & $0.05-1 \mu \mathrm{M}$
\end{tabular}




\subsubsection{Detection of proteins by indirect immunofluorescence}

Transiently transfected HeLa cells grown on coverslips were fixed with $4 \%$ formaldehyde in phosphate buffered saline (PBS) containing Hoechst 33258 (diluted 1:10000 from stock) for 15 minutes. Afterwards they were washed twice with PBS and permeabilized with $0.5 \%$ Triton-X 100 for 5 minutes. Then, the cells were incubated with $2 \%$ BSA for 10 minutes. The coverslips were transferred to a dark, wet chamber (with whatman paper and parafilm) and primary antibodies were added (see 2.1.8 for dilution). After 60-90 minutes, the coverslips were washed 3 times for 5 minutes and incubated with the secondary antibody for 45-60 minutes. The coverslips were washed again 3 times for 5 minutes, dried and mounted onto microscope slides with fluorescent mounting medium.

Phosphate buffered saline (PBS)

$140 \mathrm{mM} \mathrm{NaCl}, 2.5 \mathrm{mM} \mathrm{KCl}, 10 \mathrm{mM} \mathrm{Na}_{2} \mathrm{HPO}_{4}$, $\mathrm{KH}_{2} \mathrm{PO}_{4}(\mathrm{pH} 7.5)$

\subsubsection{Quantification of GFP-substrate distribution}

For analysis of substrate distribution in HeLa cells, 60-100 cells were counted and divided into three categories: cells with a predominant cytoplasmic staining, cells in which the substrate was equally distributed between the nucleus and the cytoplasm, and cells with a predominant nuclear staining. For graphic representation, the mean values were calculated.

Alternatively, the ratio of mean value A (\% nuclear distribution of GFP-N2c $\beta$ in the presence of RFPNup214-cNLS fragments) and mean value B (\% nuclear distribution of GFP-N2c $\beta$ in the presence of RFP-cNLS; results, section 3.3.3.2) was determined.

$$
\text { Ratio }(\mathrm{N})=\frac{\text { mean value A }(\text { RFP-Nup214-cNLS })}{\text { mean value B }(\text { RFP-cNLS })}
$$




\subsection{Molecular biology methods}

\subsubsection{Polymerase chain reaction (Mullis, 1990)}

DNA amplification was performed by the polymerase chain reaction (PCR). For a $50 \mu 1$ reaction, 50-100 ng plasmid DNA, $0.5 \mu \mathrm{M}$ of each, forward and reverse primer, $200 \mu \mathrm{M}$ dNTPs, $1 \mathrm{U}$ Vent polymerase and $1 \mathrm{x}$ Thermo buffer were used according to the manufacturer`s manual. The annealing temperature $\left(\mathrm{T}_{\mathrm{ann}}\right)$ of the primer was calculated with the Oligonucleotide properties calculator (http://www.basic.northwestern.edu/biotools/oligocalc.html).

The protocol for the Vent polymerase is given below. For purification of the PCR products, the NucleoSpin ${ }^{\circledR}$ Extract II kit was used.

$\left.\begin{array}{ccl}\text { Temperature }\left({ }^{\circ} \mathrm{C}\right) & \text { time }(\min ) & \text { cycle step } \\ \hline 95 & 5 & \text { denaturation } \\ 95 & 2 & \text { denaturation } \\ \mathrm{T}_{\text {ann. }} & 1 & \text { primer annealing } \\ 72 & 1 / \mathrm{kb} & \text { extension }\end{array}\right\} 35$ cycles

\subsubsection{Transformation and purification of plasmid DNA}

Preparation of chemical competent bacteria

$2 \mathrm{ml}$ of LB medium were inoculated with one colony of $E$. coli bacteria grown on LB agar and incubated over night at $37^{\circ} \mathrm{C}$. The culture was scaled up to $200 \mathrm{ml}$ and after reaching an $\mathrm{OD}_{600}$ of 0.5 incubated on ice for 10 minutes. The bacteria were centrifuged at $3500 \mathrm{x} \mathrm{g}$ for 10 minutes at $4{ }^{\circ} \mathrm{C}$, resuspended in ice-cold TFB I buffer and stored for 2 hours on ice. After centrifugation at $3500 \mathrm{x} g$ at $4{ }^{\circ} \mathrm{C}$, the bacteria pellet was resuspended in ice-cold TFB II buffer. $100 \mu$ l aliquots were frozen in liquid nitrogen and stored at $-80^{\circ} \mathrm{C}$.

TFB-I-buffer $100 \mathrm{mM} \mathrm{RbCl}, 15 \%$ (v/v) glycerol, $0.5 \mathrm{mM} \mathrm{LiCl}(\mathrm{pH} 5.8)$

TFB-II-buffer $10 \mathrm{mM}$ MOPS (pH 7), $10 \mathrm{mM} \mathrm{RbCl,} 75 \mathrm{mM} \mathrm{CaCl}_{2}, 15 \%$ (v/v) glycerol 


\title{
$\underline{\text { Transformation of chemical competent bacteria }}$
}

$100 \mu \mathrm{l}$ of chemical competent $E$. coli DH5 $\alpha$ bacteria were thawed on ice and $1 \mu \mathrm{g}$ of plasmid DNA was added. After incubation on ice, the bacteria were set to $42{ }^{\circ} \mathrm{C}$ for $60-90$ seconds and transferred on ice. After 2 minutes, the bacteria were supplied with $400 \mathrm{ml}$ SOC medium and shaked gently for at least 30 minutes at $37{ }^{\circ} \mathrm{C}$. $50 \mu \mathrm{l}$ of the whole suspension was plated on LB agarose containing ampicillin or kanamycin.

\section{Midi preparation}

100-200 ml LB culture was inoculated with a single colony from an LB agar plate at $37{ }^{\circ} \mathrm{C}$ over night. The bacteria were centrifuged for 10 minutes at $4000 \mathrm{rpm}$. For purification of the plasmid DNA, the NucleoBond ${ }^{\circledR}$ PC100 or PC500 kit was used.

\section{$\underline{\text { Mini preparation }}$}

Single colonies from an agar plate were transferred to $3 \mathrm{ml} \mathrm{LB}$ medium and grown over night at $37^{\circ} \mathrm{C}$. $1.5 \mathrm{ml}$ of the culture was centrifuged for 5 minutes at $13000 \mathrm{rpm}$, and the bacteria pellet was resuspended in $100 \mu \mathrm{l}$ P1 buffer. The cells were lysed with $200 \mu \mathrm{P} 2$ buffer for 5 minutes at room temperature. After neutralization of the suspension with $150 \mu \mathrm{l}$ P3 buffer for 15 minutes on ice, the lysate was centrifuged for 15 minutes at $4{ }^{\circ} \mathrm{C}$. The supernatant containing the plasmid DNA was collected, and $100 \%(\mathrm{v} / \mathrm{v})$ ethanol was added to precipitate the DNA. After centrifugation for 15 minutes at $4{ }^{\circ} \mathrm{C}$, the supernatant was discarded, the pellet was washed with $70 \%$ (v/v) ethanol, dried and dissolved in TE buffer $\mathrm{pH}$ 7.5. Plasmid DNA was stored at $-20{ }^{\circ} \mathrm{C}$.

\author{
P1 buffer \\ 50 mM Tris- $\mathrm{HCl}(\mathrm{pH}$ 8), 10 mM EDTA, $100 \mu \mathrm{g} / \mathrm{ml}$ RNase A \\ P2 buffer \\ $200 \mathrm{mM} \mathrm{NaOH}, 1 \%$ (v/v) SDS \\ P3 buffer \\ 3 M KOAc (pH 5.3) \\ 1x TE buffer \\ $10 \mathrm{mM}$ Tris pH 7.5, 1 mM EDTA
}




\subsubsection{Agarose gel electrophoresis}

Depending on the size of the DNA fragments, 0.8- 2.0\% agarose gels were prepared with $1 \mathrm{x}$ TAE buffer and additional DNA stain (SERVA DNA stain G, diluted 1:100000). Restricted plasmid DNA or PCR products were supplied with 1x DNA sample buffer and loaded on the agarose gel and 80-130 V was applied, depending on the size of the agarose gel. Afterwards, the gel was analyzed with UV light.

50x TAE buffer $2 \mathrm{M}$ Tris, 0.05 M EDTA pH 8, 5.8\% acetic acid

\subsubsection{Restriction of DNA by endonucleases}

Restriction of plasmid DNA and DNA fragments was performed according to the manufacturer's manual. For analytical and preparative digestion, 2-4 $\mu \mathrm{g}$ plasmid DNA were incubated with appropriate amounts of enzymes and buffers in a final volume of $20 \mu \mathrm{l}$ at $37{ }^{\circ} \mathrm{C}$ for $1-2$ hours. DNA fragments were separated and analyzed by agarose gel electrophoresis. For ligation, DNA was cut out from the agarose gel with a scalpel and purified with the NucleoSpin ${ }^{\circledR}$ Extract II kit.

\subsubsection{Ligation of DNA fragments}

Purified vector DNA and the insert DNA were added to the $10 \mu$ ligation reaction in a 1:3 ratio. In general, $10 \mathrm{ng}$ vector DNA were used. The corresponding amounts of the insert DNA were calculated with a ligation calculator (http://www.insilico.uni-duesseldorf.de/Lig_Input.html). The sample reaction, containing $1 \mathrm{x}$ ligase buffer, $0.5 \mu \mathrm{M}$ ATP and $0.5 \mu \mathrm{T} 4$ ligase (Fermentas), was incubated over night at $16{ }^{\circ} \mathrm{C}$. The complete sample was transformed into E. coli $\mathrm{DH} 5 \alpha$. 


\subsubsection{Oligonucleotide cloning}

For insertion of short DNA fragments up to $60 \mathrm{bp}$ into a vector, complementary oligonucleotides with overhanging restriction sites were ordered. These restriction sites fit accurately into a vector digested with the corresponding enzymes. $100 \mu \mathrm{M}$ of the oligonucleotide were incubated in $50 \mu \mathrm{l}$ annealing buffer for 3 minutes at $95{ }^{\circ} \mathrm{C}$. After that, the mixture was cooled down slowly and was incubated for 1 hour at $37^{\circ} \mathrm{C}$. In contrast to a PCR product, the annealed oligonucleotides are unphosphorylated. To allow efficient ligation with the vector, $5 \mu \mathrm{l}$ of the annealed oligonucleotide were incubated with $10 \mathrm{U} \mathrm{T} 4$ polynucleotide kinase and $200 \mu \mathrm{M}$ ATP for 30 minutes at $37^{\circ} \mathrm{C}$. The reaction was stopped by heating the sample for 10 minutes at $65^{\circ} \mathrm{C}$.

Annealing buffer $(2 \mathrm{x})$

$100 \mathrm{mM}$ potassium acetate, $30 \mathrm{mM}$ HEPES $\mathrm{pH} 7.4,2 \mathrm{mM}$

magnesium acetate

\subsubsection{DNA-sequencing}

Sequencing of DNA was performed by either myGATC (Konstanz) or at the GZMB by using a sequence analyzer (Genetic Analyzer 3100; Applied Biosystems). For the latter, the sequencing reactions were prepared in the laboratory. 200-400 ng plasmid DNA, 10 pmol primers, $1 \mu 1$ sequencing mix, $2 \mu \mathrm{l}$ sequencing buffer and sterile water were mixed in $10 \mu \mathrm{l}$ final volume. Plasmid DNA was amplified in a PCR program as listed below. Afterwards, $1 \mu$ of each, $3 \mathrm{M} \mathrm{Na}(\mathrm{OAc}) \mathrm{pH} 5.2$ and $125 \mathrm{mM}$ EDTA, and $50 \mu \mathrm{l}$ ice-cold ethanol (100\%) was added and incubated for 5 minutes at room temperature. The samples were centrifuged for 15 minutes at $13000 \mathrm{rpm}$ and $4{ }^{\circ} \mathrm{C}$ and the supernatant was removed. The pellet was washed once with $70 \%(\mathrm{v} / \mathrm{v})$ ethanol, dried and dissolved in $15 \mu \mathrm{l}$ formamide.

$\left.\begin{array}{cccl}\text { PCR cycle protocol } & \text { Temperature }\left({ }^{\circ} \mathrm{C}\right) & \text { time }(\mathrm{s}) & \text { cycle step } \\ & 96 & 120 & \text { denaturation } \\ 99 & 10 & \text { denaturation } \\ & 55 & 15 & \text { primer annealing } \\ 60 & 240 & \text { extension }\end{array}\right\} 25$ cycles




\section{Results}

\subsection{In vivo analysis of CRM1, CRM1-fragments and chimeras}

At the onset of the work for this thesis, CRM1-fragments were constructed and analyzed to investigate the function of distinct regions. The CRM1 structure was only available for the $\mathrm{C}$-terminus, which is composed of HEAT repeat motifs (Petosa et al., 2004, see Fig. 8). The Ran binding region to CRM1 was not exactly determined and was based on sequence similarities to other transport factors like

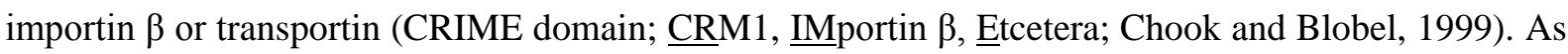
the function for the C-terminal part of CRM1 was not known, we used CRM1-fragments that were truncated at the C-terminus. During the course of this project, the crystal structure of the trimeric export complex was solved (Monecke et al., 2009, see introduction), showing CRM1 as a ring-like protein. To maintain the ternary structure of full-length CRM1, chimeras consisting of $H$. sapiens (Homo sapiens) and C. elegans (Caenorhabditis elegans) CRM1 were constructed and analyzed. C. elegans CRM1 is $60 \%$ homologous to H. sapiens CRM1. Alignments revealed that especially the NES-binding region is almost identical to that of human CRM1. Furthermore, C. elegans CRM1 is able to interact with human RanGTP and an NES-peptide (Roloff, Diploma thesis). Concluded from that, CRM1-chimeras consisting of H. sapiens and C. elegans CRM1 should fulfil all requirements for the assembly of the trimeric export complex in the nucleus of mammalian cells. One striking difference to H. sapiens CRM1 is the inability of $C$. elegans CRM1 to interact with human nucleoporins (Roloff, 2008, Diploma thesis).
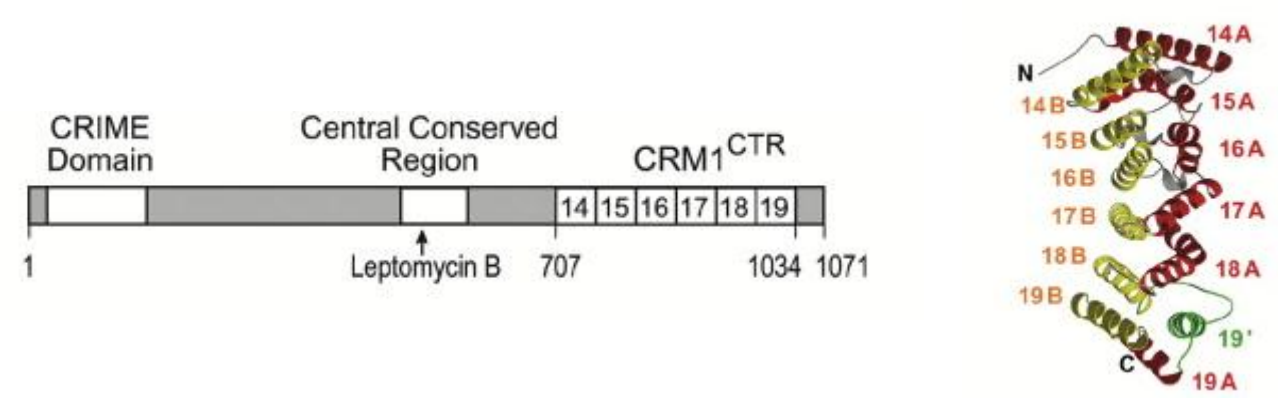

Fig 8. Scheme of CRM1 structure. In this view, CRM1 consists of an N-terminal CRIME-domain, which corresponds to the Ran (switch II) -binding region in transportin 1 and importin $\beta$ (Fornerod et al., 1997). The central conserved region (in accordance to the current model) contains the RanGTP-dependent NES recognition site. The numbers in the C-terminal region represent the tandem HEAT repeats 14-19 (Petosa et al., 2004). 


\subsubsection{Distribution of nuclear export cargos after Leptomycin B treatment of HeLa cells}

Leptomycin B (LMB) is an unsaturated fatty acid with a terminal $\delta$-lactone ring. It covalently modifies cysteine 528 in CRM1, thus preventing cargo binding. As a consequence, the trimeric export complex cannot assemble, and export cargos remain in the nucleus. In order to identify suitable export cargos for further analysis, HeLa cells were transfected with plasmids coding for different proteins and treated with LMB. This approach had already been used for GFP-NES (Roloff, 2008, Diploma thesis), and was now extended for quantification of GFP-SPN1 (snurportin 1), GFP-NC2 $\beta$ (negative cofactor $2 \beta$ ), GFP-Nmd3 and HA-DDX3. SPN1 is an $\mathrm{m}_{3} \mathrm{G}$-cap receptor and imports $\mathrm{m}_{3} \mathrm{G}$-capped uridine-rich small nuclear RNPs (U snRNPs; Huber et al., 1998). It has an importin $\beta$ binding (IBB) domain but it does not share the structural similarity to the importin $\alpha$ arm repeat domain. SPN1 is recycled back to the cytoplasm with the help of CRM1 in a trimeric export complex (Paraskeva et al., 1999). The transcription factor $\mathrm{NC} 2 \beta$ forms a heterodimer with $\mathrm{NC} 2 \alpha$ and regulates the polymerase II transcription machinery by either repression or activation of transcription (Geisberg et al., 2001; Cang et al., 2002). NC2 $\beta$ contains a classical nuclear localization signal (NLS) as well as an NES comparable to other known export cargos. Furthermore, it interacts with importin $\alpha / \beta$ and CRM1 and is sensitive to LMB treatment (Kahle et al., 2009). Nmd3 acts as an adapter protein between the 60S preribosomal subunit and CRM1 (Thomas and Kutay, 2003; Trotta et al., 2003). It also contains basic and non-basic regions (but no classical NLS) mediating nuclear import. DDX3 belongs to the DEAD (D-E-A-D = Asp-Glu-Ala-Asp) box RNA helicase family. It facilitates nuclear export of HIV RNAs via CRM1 and also shuttles back to the nucleus (Yedavalli et al., 2004).

After transfection and LMB treatment, HeLa cells were fixed and analyzed by immunofluorescence microscopy. In untreated cells, GFP-NES was located in the cytoplasm, but in cells treated with LMB it accumulated in the nucleus (see Fig. 9A). As the construct does not contain an NLS, it is still able to diffuse freely in the cell. Hence, there was also a slight cytoplasmic signal for GFP-NES after LMB treatment (see arrow). The distribution of the other tested proteins is shown in Fig. 9B. As expected, localization of all of them was affected by LMB, though the extent of accumulation differed from protein to protein. In nearly all cells, GFP-NES, GFP-NC2 $\beta$ and GFP-SPN1 were located in the nucleus, whereas there was only a mild effect of LMB on GFP-Nmd3 (approximately 50\% of the cells showed nuclear accumulation). The amount of cells with cytoplasmic HA-DDX3 was reduced from $100 \%$ to only $65 \%$ after LMB treatment.

As GFP-NES, GFP-NC2 $\beta$ and GFP-SPN1 showed the strongest LMB-effect, they were chosen for further analysis of CRM1 and also used in the second part of this thesis (section 3.2). 
(A)
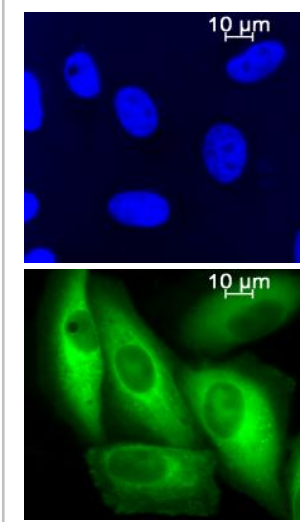

- LMB

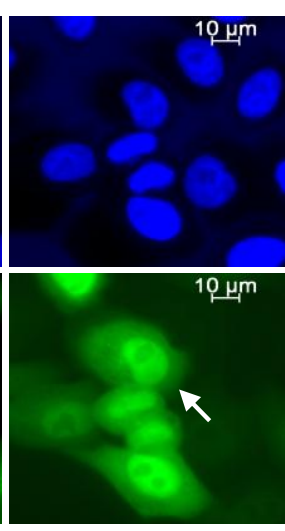

$+\mathrm{LMB}$
Fig. 9 LMB effect on CRM1-dependent export cargos. (A) HeLa cells were transfected with GFP-fused NES, NC2 $\beta$, SPN1, Nmd3 or with HADDX3. After 48 hours the cells were treated with LMB $(5 \mathrm{nM})$ for 3 hours. The cells were fixed and the distribution of the proteins was analyzed by fluorescence microscopy. HA-DDX3 was detected with an anti-HA-antibody. (B) The LMB effect was quantified by counting at least 100 cells per sample. The distribution of the proteins in the cells is indicated in \% ( $\mathrm{N}$ nuclear, = equally located between nucleus and cytoplasm, $\mathrm{C}$ cytoplasmic).

(B)

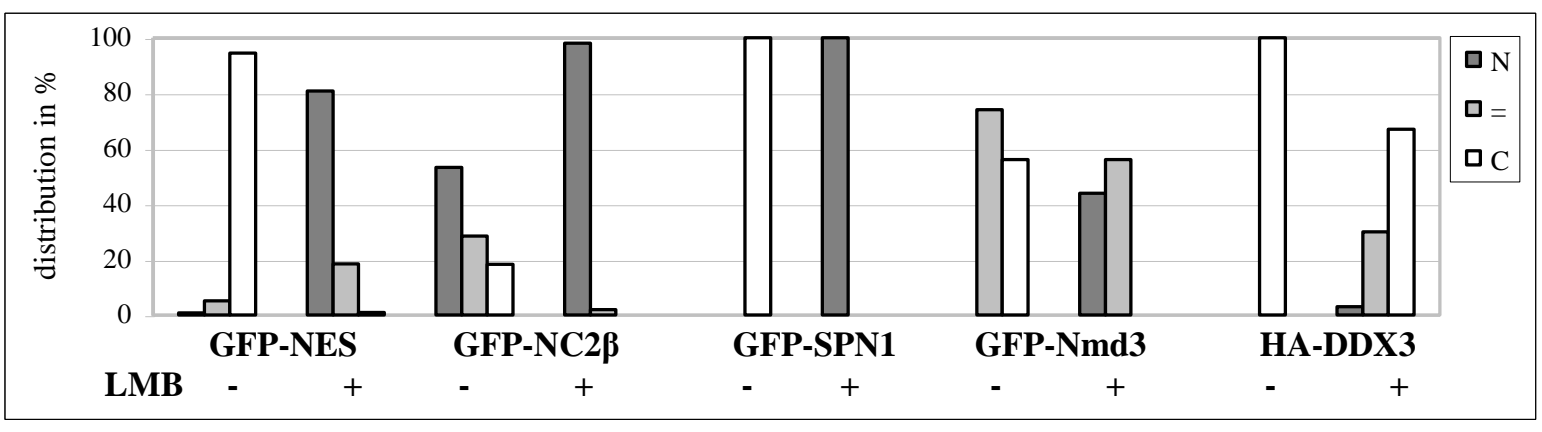

\subsubsection{The CRM1 mutant C528S can overcome the LMB effect on several export}

\section{cargos}

The LMB approach is an effective tool to analyze the localization of CRM1-dependent export cargos. To investigate exogenous CRM1 constructs in mammalian cells, a point mutation was introduced, preventing modification of CRM1 by LMB. CRM1 C528S is still active as an export receptor (Roloff, 2008, Diploma thesis). By adding LMB to cells that express the HA-CRM1 C528S, endogenous CRM1 is modified, but not the mutant. This allows functional analysis of CRM1 under conditions where endogenous CRM1 is eliminated. The mutagenesis of CRM1 C528S and the analysis of GFP-NES distribution had been performed previously (Roloff, 2008, Diploma thesis). This approach was now extended to GFP-SPN1 and GFP-NC2 $\beta$. The GFP-fused cargos were coexpressed in HeLa cells with either HA-CRM1 wildtype (WT) or CRM1 C528S. After two days, the cells were treated with LMB and analyzed. CRM1 C528S showed a similar nuclear and rim staining as CRM1 WT, which indicates the association of both proteins with the nuclear pore complex (see Fig. 10A). After LMB treatment of the cells overexpressing HA-CRM1 WT, GFP-NES accumulated in the nucleus (see arrow) and this was also observed for GFP-SPN1 and GFP-NC2 $\beta$ (data not 
shown). Not only endogenous CRM1 is modified by LMB but also the transfected HA-CRM1. In the presence of HA-CRM1 C528S, GFP-NES was located in the cytoplasm, and this localization was nearly unaffected after LMB treatment (Fig. 10A, right panel, see arrow; and Fig. 10B).

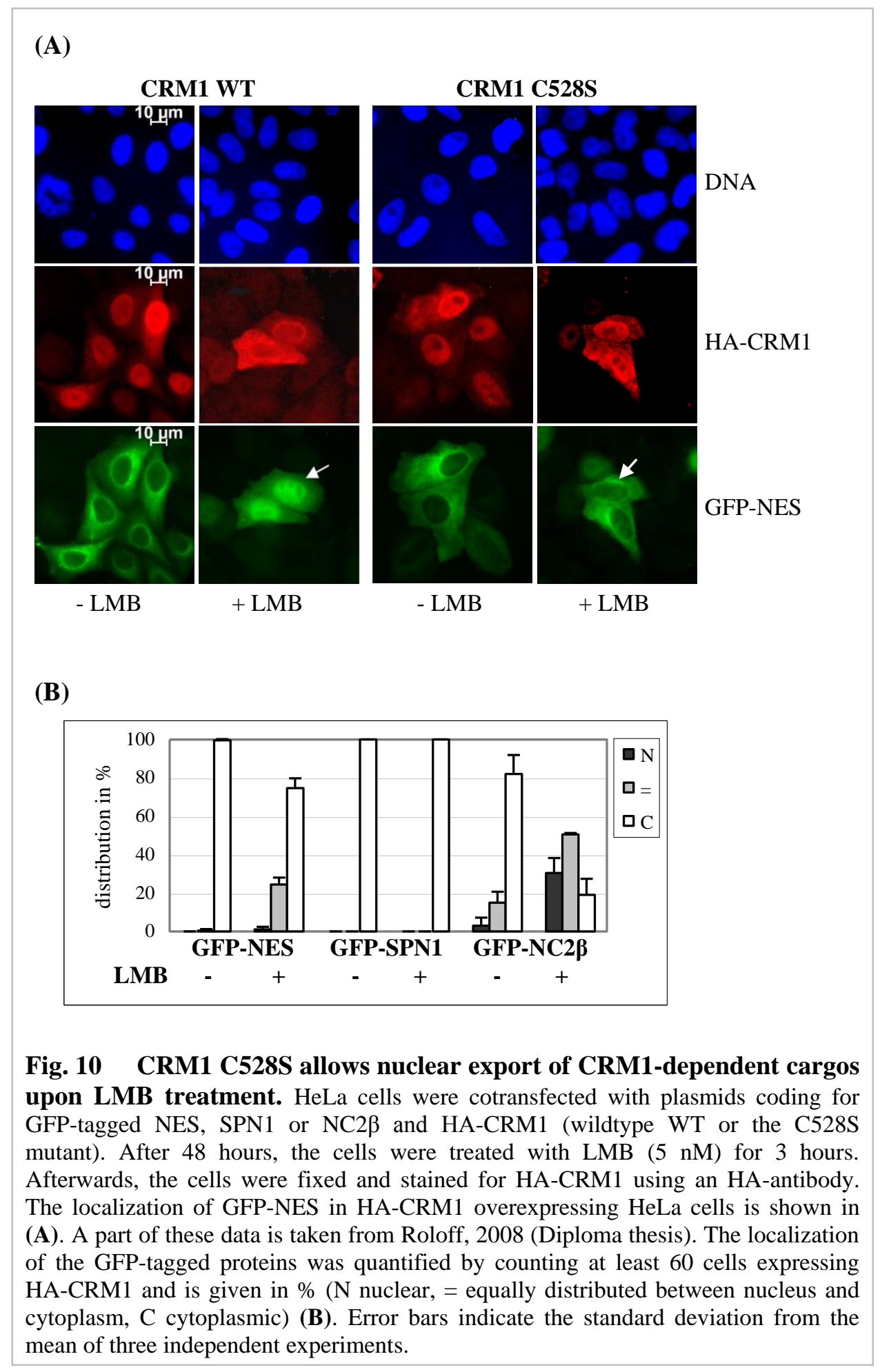

Basically, HA-CRM1 C528S had the same effect on localization of GFP-SPN1 and GFP-NC2 3 . GFPSPN1 was cytoplasmic in all HeLa cells co-expressing the HA-CRM1 mutant, and after LMB 
treatment the cargo was still in the cytoplasm (Fig. 10B). Without LMB, GFP-NC2 $\beta$ was mainly cytoplasmic (80\% of the cells) in the presence of the CRM1 mutant. In cells treated with LMB, GFP$\mathrm{NC} 2 \beta$ distributed mostly equally between the nucleus and the cytoplasm. Compared to localization of GFP-NC2 $\beta$ alone (Fig. 9B), it becomes clear that CRM1 is rate-limiting for nuclear export of the cargo. This has previously been shown for artificial reporter substrates (Waldmann et al., 2012).

Together, the CRM1 C528S mutant is functional, and combined with LMB it is an effective tool for analysis of CRM1 variants.

\subsubsection{CRM1-fragments are not able to mediate nuclear export of proteins}

CRM1-fragments containing the C528S mutation were constructed and analyzed in order to specify regions for interaction partners of CRM1 and to understand which regions in CRM1 are important for its function in nuclear export. According to Petosa et al. (2004), the N-terminal region of CRM1 was thought to have the Ran-binding capability and the central region was implicated in export cargo binding, but the function of the C-terminus of CRM1 remained unclear. Moreover, the binding region(s) for nucleoporins like Nup214 in CRM1 are not known.

\subsubsection{Localization of CRM1-truncations}

Full-length CRM1 associates with the nuclear pore, but it was not known where CRM1-fragments would localize in the cell. To test this, plasmids coding for CRM1-fragments containing the C528S mutation were constructed (Fig. 11A) and used for transfection of HeLa cells. After two days, the cells were fixed and stained (Fig. 11B). Whereas CRM1 containing HEAT repeats (HR) 1-13 showed a considerable amount of cytoplasmic staining (see asterisk), CRM1 HR 1-16 and 1-18 were almost completely in the nucleus with a clear boundary to the cytoplasm. This suggests that the ability of CRM1 to locate to the nucleus increases with the number of HEAT repeats. However, only full-length CRM1 showed a rim staining around the nuclear envelope (see arrow). This points to the association of full-length CRM1 with the nuclear pore and thus, to the interaction of CRM1 with FG-rich nucleoporins like Nup214, Nup153 or p62. This led to the assumption that the tested CRM1fragments might not be able to interact with nucleoporins. In coimmunoprecipitation experiments the binding between endogenous nucleoporins and the HA-CRM1-truncations was analyzed but no interaction could be detected, in contrast to full-length HA-CRM1 (data not shown). As RanGTP enhances the association of CRM1 to nucleoporins, the binding of these fragments to nucleoporins could be indirectly affected due to disturbed Ran interaction. Hence, the binding regions for 
nucleoporins could be located at the N-terminal or central part of CRM1 but cannot be detected by in vitro binding studies, using CRM1-fragments.

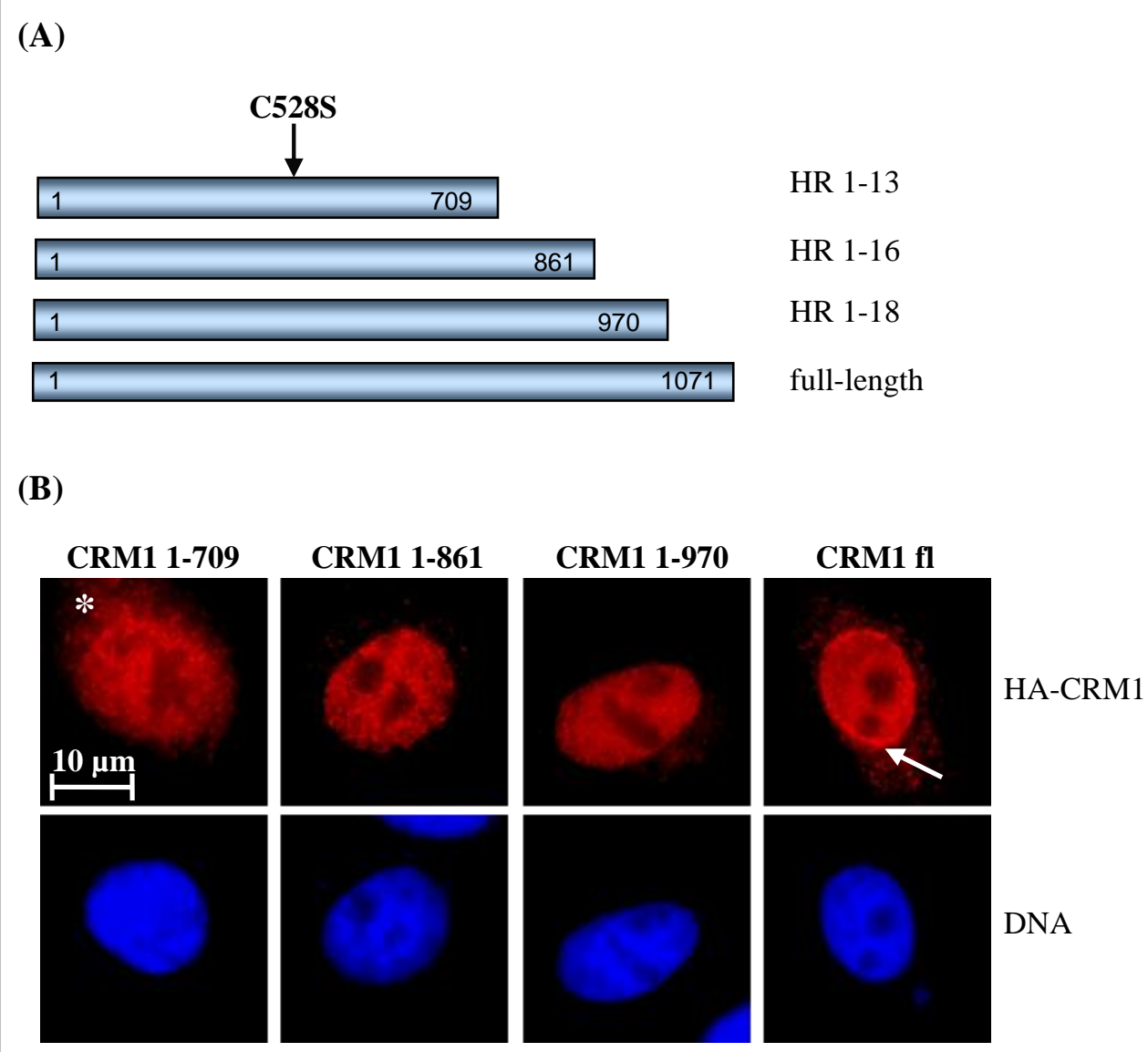

Fig. 11 Only full-length CRM1 is concentrated at the nuclear pore.

(A) Schematic view of CRM1-truncations. The CRM1-fragments used in this work were constructed according to the C-terminal structure that was available at that time (Petosa et al., 2004). The numbers in the bars represent amino acid boundaries. HR 1-13, 1-16 and 1-18 indicate the number of the residual N-terminal HEAT repeats. (B) HeLa cells were transfected with full-length or C-terminal truncated HA-CRM1. After 48 hours, the cells were fixed and stained using an HA- antibody. The cells were analyzed by immunofluorescence microscopy. CRM1 1-709 shows considerable amounts of cytoplasmic staining (see asterisk). The arrow indicates a rim staining that is only present in cells overexpressing full-length CRM1. 


\subsubsection{Only full-length CRM1 is able to mediate export through the nuclear pore}

In order to test the export capability of the HA-CRM1 C528S fragments, HeLa cells were cotransfected with plasmids coding for GFP-NES and the CRM1-fragments as indicated in Fig 11A. After two days, the cells were treated with LMB. Only full-length CRM1 was able to transport GFPNES out of the nucleus (Fig. 12, see arrow) whereas in all cells overexpressing CRM1-fragments, GFP-NES accumulated in the nucleus. As already mentioned in the section above, the C-terminus of CRM1 might be required for proper ring closure. In turn, a disturbed ring structure of CRM1 could be the reason for the inability of CRM1-fragments to mediate nuclear export.

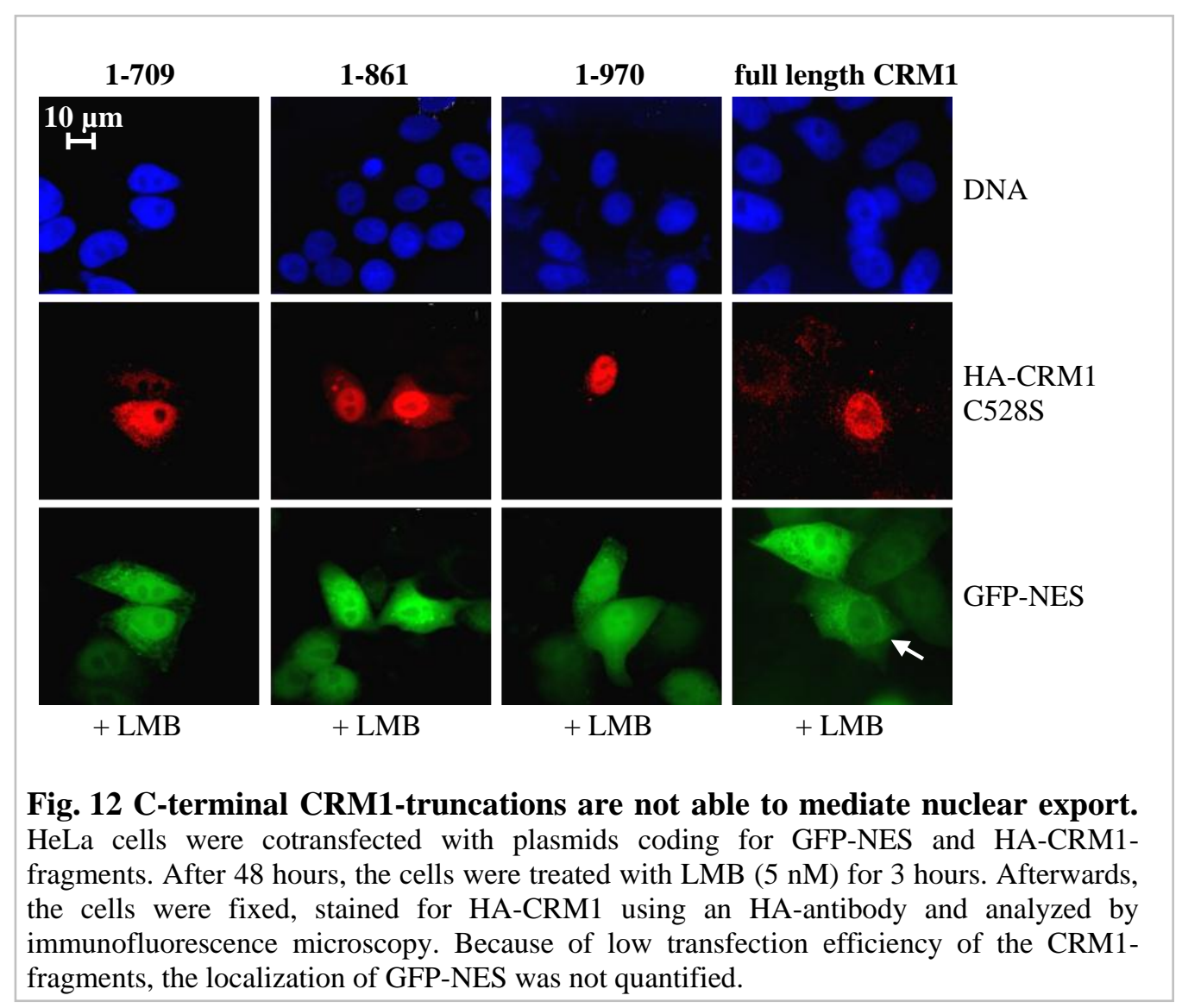




\subsubsection{CRM1-chimeras as an alternative approach to study export capabilities in vivo}

The previously solved structure of the CRM1-RanGTP-cargo complex (Monecke et al., 2009), showing CRM1 as a ring-like protein, indicated why CRM1-fragments are not functional in nuclear export. The structure revealed a complex binding of RanGTP to several HEAT repeats at the N-and at the C-terminus of CRM1. Regarding this, RanGTP binding to CRM1-fragments lacking the C-terminus might indeed be affected. Hence, there might be problems with forming a functional trimeric export complex and also binding of CRM1 to nucleoporins could be disturbed. To avoid such problems, full-length chimeras consisting of $H$. sapiens and $C$. elegans CRM1 were constructed accordingly to the secondary structure. The usage of $H$. sapiens/C. elegans CRM1-chimeras allows the following assumptions:

1. The ring structure of CRM1 stays preserved.

2. The cooperativity of CRM1-Ran binding is not disturbed.

3. The CRM1-binding and the release of the export cargo can occur.

\subsubsection{C. elegans CRM1 promotes export of a simple GFP-NES, but is unable to export more complex cargos out of the nucleus}

To test whether C. elegans CRM1 is functional in nuclear export in human cells, HeLa cells were cotransfected with plasmids coding for $C$. elegans HA-CRM1 C538S (corresponding to the C528S mutation in H. sapiens CRM1) and GFP-NES, GFP-SPN1 or GFP-NC2 $\beta$, respectively. After two days, the cells were treated with LMB. The localization of GFP-NES in HeLa cells in the presence of C. elegans CRM1 was already investigated in Roloff, 2008 (Diploma thesis). The quantification of GFP-NES distribution was completed in this work and extended to other GFP-substrates. In cells expressing C. elegans HA-CRM1 mutant, all tested GFP-cargos were mostly located in the cytoplasm (Fig. 13). After LMB treatment, GFP-NES distributed equally between the nucleus and the cytoplasm in $80 \%$ of the cells. Thus, C. elegans CRM1 promotes export of GFP-NES. Strikingly, export of more complex cargos was not supported by $C$. elegans CRM1, as GFP-SPN1 and GFP-NC2 $\beta$ accumulated in all cells overexpressing C. elegans CRM1 after LMB treatment. In cells expressing H. sapiens CRM1 C528S (see Fig. 10A and B), LMB had only a very weak effect on the distribution of all investigated cargos. By contrast, the LMB effect in cells expressing C. elegans CRM1 was mild on GFP-NES, but very strong on distribution of GFP-SPN1 and NC2 $\beta$. We know from the crystal structure of the trimeric export complex that SPN1 binds to the NES-groove, but also to other sites in 
CRM1. Probably C. elegans CRM1 is able to form a trimeric export complex with the NES, but is lacking other binding sites for SPN1. The binding mechanism of NC2 $\beta$ to CRM1 is not known.

Aside from that, coimmunoprecipitation experiments showed that C. elegans CRM1 is not able to interact with the human nucleoporins Nup214 and p62 (Roloff, 2008, Diploma thesis). This might be another reason why the export capability is restricted for C. elegans CRM1 in mammalian cells.

Based on these data, the usage of chimeras consisting of human and C. elegans CRM1 could give more detailed information about regions in $H$. sapiens CRM1 that are required for nuclear export of proteins.

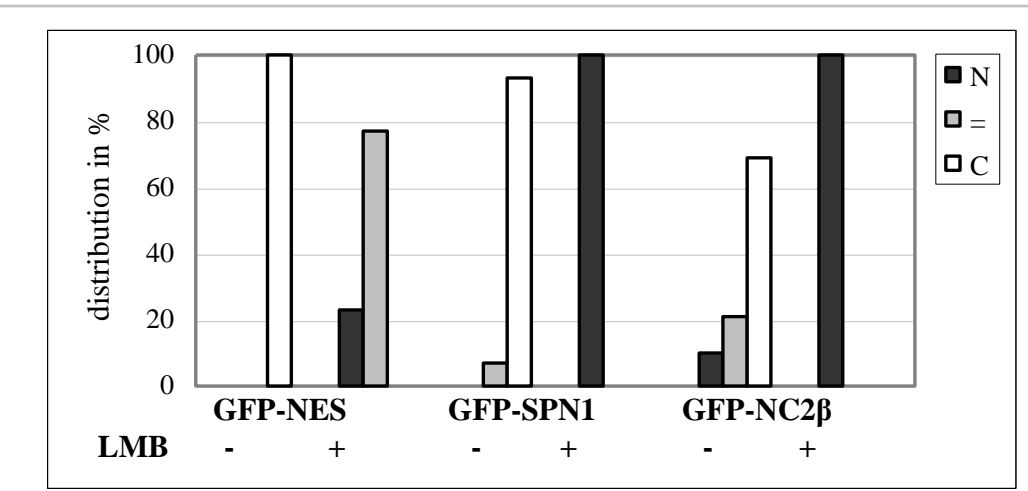

Fig. 13 C. elegans CRM1 promotes nuclear export of GFPNES, but not of GFP-SPN1 and GFP-NC2及. HeLa cells were cotransfected with plasmids coding for $C$. elegans HA-CRM1 C538S and GFP-NES, GFP-SPN1 or GFP-NC2 $\beta$, respectively. After 48 hours, the cells were treated with LMB $(5 \mathrm{nM})$ for 3 hours. Afterwards, the cells were fixed, stained for HA-CRM1 using an HA-antibody and analyzed by immunofluorescence microscopy. The localization of the GFP-substrates in HA-CRM1 expressing cells is given in \%. At least 60 cells were counted $(\mathrm{N}$ nuclear, $=$ equally distributed between nucleus and cytoplasm, C cytoplasmic). 


\subsubsection{CRM1-chimeras differ in their localization}

C. elegans CRM1 is 60\% homologous to human CRM1 and lacks binding sites for human Nup214 and p62 (Roloff, 2008, Diploma thesis). By combining portions of $H$. sapiens and C. elegans CRM1 parts, we addressed the question of which part of CRM1 is mediating the association with the nuclear pore. H. sapiens/C. elegans CRM1-chimeras were constructed as depicted in Fig. 14A. Type I chimeras are a mixture of $H$. sapiens N-terminal HR 1-11 and C. elegans C-terminal HR 12-21, and vice versa. As HR 11 and 12 are 90\% identical in H. sapiens and C. elegans CRM1, there is a smooth transition between the moieties (see appendix). Chimeras of type II contain a shorter N-terminus (HR 1-8) from one species and a longer C-terminus (9-21) from the other species.

HeLa cells were transfected with plasmids coding for $H$. sapiens and C. elegans CRM1 and the chimeras, respectively, and the localization of CRM1 was analyzed. H. sapiens HA-CRM1 showed the typical rim staining as seen for endogenous CRM1 (Fig. 14B and C). By contrast, C. elegans CRM1 was located homogeneously in the cell and even showed a stronger accumulation in the cytoplasm (see asterisk). This result also indicates the inability of C. elegans CRM1 to interact with nucleoporins. Only chimera type I with the human part at the N-terminus and the $C$. elegans part at the C-terminus ( $\mathrm{HsCe} \mathrm{I}$ ) was located at the nuclear envelope (see arrows) similar to full-length human CRM1. Thus, the N-terminal half of CRM1 (HR 1-11) seems to be required for nuclear pore association.

Comparing the localization of $\mathrm{HsCe}$ II (H. sapiens HR 1-8, C. elegans HR 9-21) and CeHs II (vice versa), both chimeras lack a rim-staining around the nuclear envelope and are equally distributed between the nucleus and the cytoplasm. Notably, $\mathrm{CeHs}$ II localized similar to full-length $\mathrm{C}$. elegans CRM1, whereas $\mathrm{HsCe}$ II showed a smooth staining in the nucleus, and a rather diffuse staining in the cytoplasm (see arrows).

Considering CRM1-chimera $\mathrm{HsCe} \mathrm{I}$, which was associated with the nuclear pore, and chimera $\mathrm{HsCe}$ II, which localized to the nucleus and the cytoplasm without a rim-staining, there were only 3 HEAT repeat (HR 9, 10 and 11) exchanges required to get this dramatic effect on localization. These HEAT repeats are highly conserved between $H$. sapiens and C. elegans CRM1 (aa 404 until 550 in human CRM1, see appendix). Nevertheless, this region could be responsible for association of human CRM1 with the nuclear pore. In coimmunoprecipitation experiments, the interaction of the chimeras with endogenous nucleoporins was tested, but no binding could be detected (data not shown). For further analysis, it will be required to perform binding studies with recombinant CRM1-chimeras. 


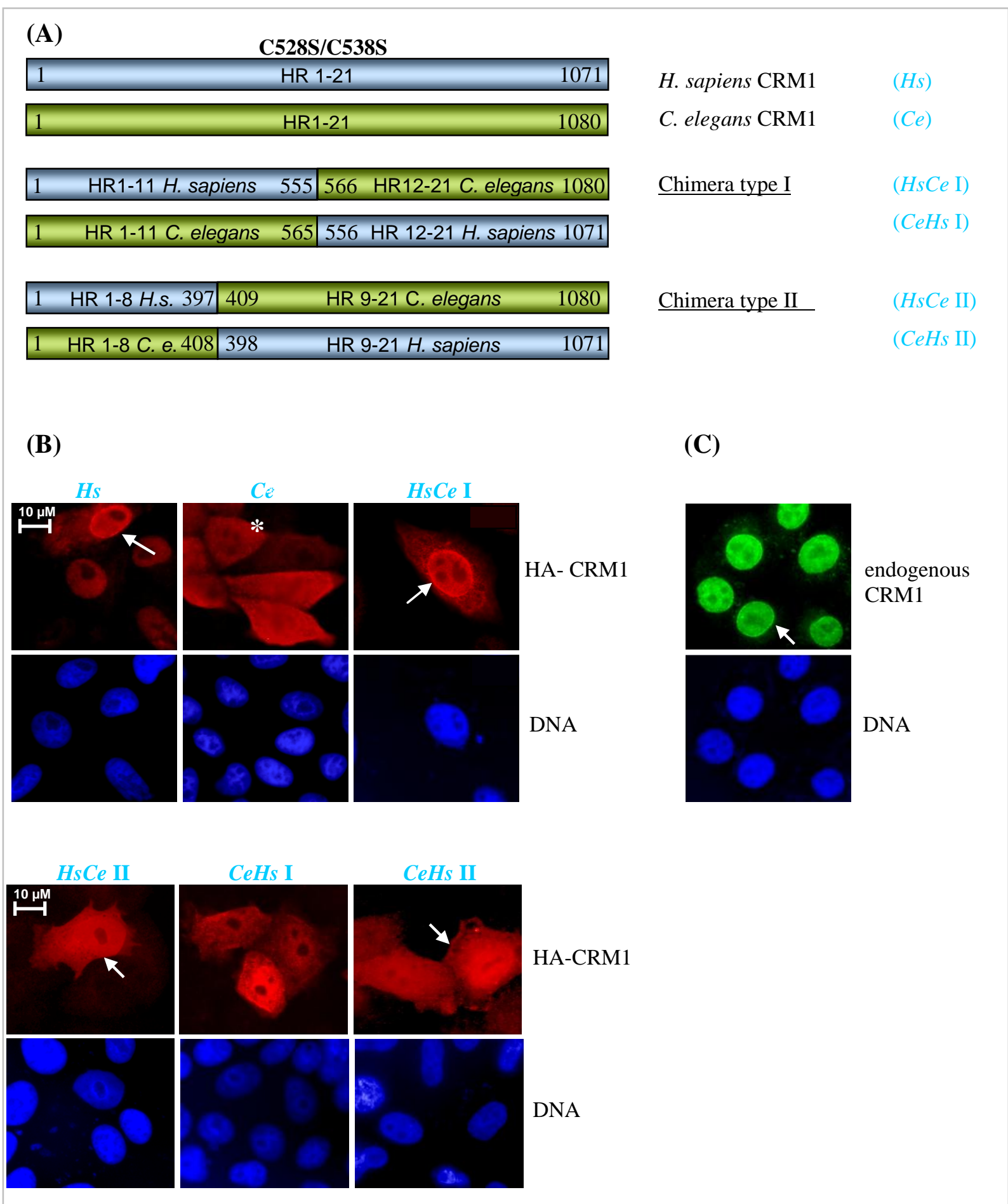

Fig. 14 The CRM1-chimera $\mathrm{HsCe}$ I associates with the nuclear envelope comparable to fulllength CRM1. (A) Schematic view of CRM1 and CRM1-chimeras. H. sapiens CRM1 (blue) and C. elegans CRM1 (green) were constructed as indicated. A cloning strategy was followed to maintain the secondary structure of the proteins. The chimeras of type I consist of N-terminal HR 1-11 and C-terminal HR 12-21, corresponding to the available CRM1-structure (Monecke et al., 2009). The type II chimeras contain HR 1-8 and 9 to 21 of CRM1. The numbers underneath the bars indicate the amino acid residues. (B) HeLa cells were transfected with human/C. elegans CRM1 or with CRM1-chimeras as indicated. After 48 hours, the cells were fixed and stained for HA-CRM1 using an HA-antibody. (C) HeLa cells were fixed and stained for endogenous CRM1. 


\subsubsection{The export capability of CRM1-chimeras correlates with their localization in HeLa cells}

To investigate the functionality of the CRM1-chimeras (containing the C528S/C538S mutation), they were cotransfected with plasmids coding for the GFP-fused substrates NES, SPN1 and NC2 $\beta$ in HeLa cells. After two days, the cells were treated with LMB to inhibit endogenous CRM1 and analyzed by immunofluorescence microscopy. In cells expressing functional HA-CRM1, LMB is expected to have no or very mild effects in the localization of cargo-proteins, since the exogenous CRM1 is insensitive to the drug. The localization of GFP-NES was cytoplasmic in HeLa cells co-expressing CRM1chimera $\mathrm{HsCe} \mathrm{I}$, and after LMB treatment GFP-NES was still cytoplasmic in $70 \%$ of the cells (Fig. 15A and B). The cytoplasmic localization of GFP-SPN1 was not affected at all in LMB-treated cells. Strikingly, GFP-NC2 $\beta$ accumulated in the nucleus in the presence of chimera $H s C e$ I after LMB treatment, suggesting a specific binding mechanism to CRM1 as described for SPN1. In contrast to CRM1-chimera $\mathrm{HsCe} \mathrm{I}$, all tested GFP-cargos accumulated in the nucleus in the presence of the chimera $\mathrm{CeHs}$ I and LMB treatment, suggesting that the N-terminal portion of CRM1 is required for CRM1-functions in mammalian cells.

LMB-treated HeLa cells overexpressing chimeras type II (Fig. 16A and B) showed equal distribution of GFP-NES between the nucleus and the cytoplasm (and nuclear GFP-NC2 $\beta$, see appendix, Fig. S1). Therefore, these chimeras are clearly restricted in their export capability. Interestingly, in LMBtreated cells overexpressing the CRM1-chimera $\mathrm{HsCe}$ II, the export cargo had a similar distribution pattern like the chimera itself (see asterisk).

In general, the localization of the chimeras correlates with their ability to promote nuclear export. Based on this, the association with the nuclear envelope could be required for CRM1 to fulfill its function as an export receptor in mammalian cells. With respect to cargo interaction, the C-terminus of . elegans CRM1 (HR 1-11) can substitute for the C-terminus of H. sapiens CRM1 for SPN1 or a simple NES in mammalian cells, but not for NC2 $\beta$.

In summary, only the CRM1-chimera $\mathrm{HsCe}$ I associated with the nuclear envelope and was able to promote nuclear export in mammalian cells for a subset of proteins (Table 2). The ability for association with the nuclear envelope (NE) of this chimera got lost by the exchange of $H$. sapiens HRs 9-11 into C. elegans CRM1 HRs ( $\mathrm{HsCe} \mathrm{I} \rightarrow \mathrm{HsCe}$ II), but this could not be reconstituted by extension of the human part of CRM1 C-terminally ( $\mathrm{CeHs} \mathrm{I} \rightarrow \mathrm{CeHs}$ II). Taken together, HRs 9-11 of CRM1 seem to be required for nuclear pore association, but there must be additional regions in human CRM1 HR 1-7 that are required for functional nuclear export. 


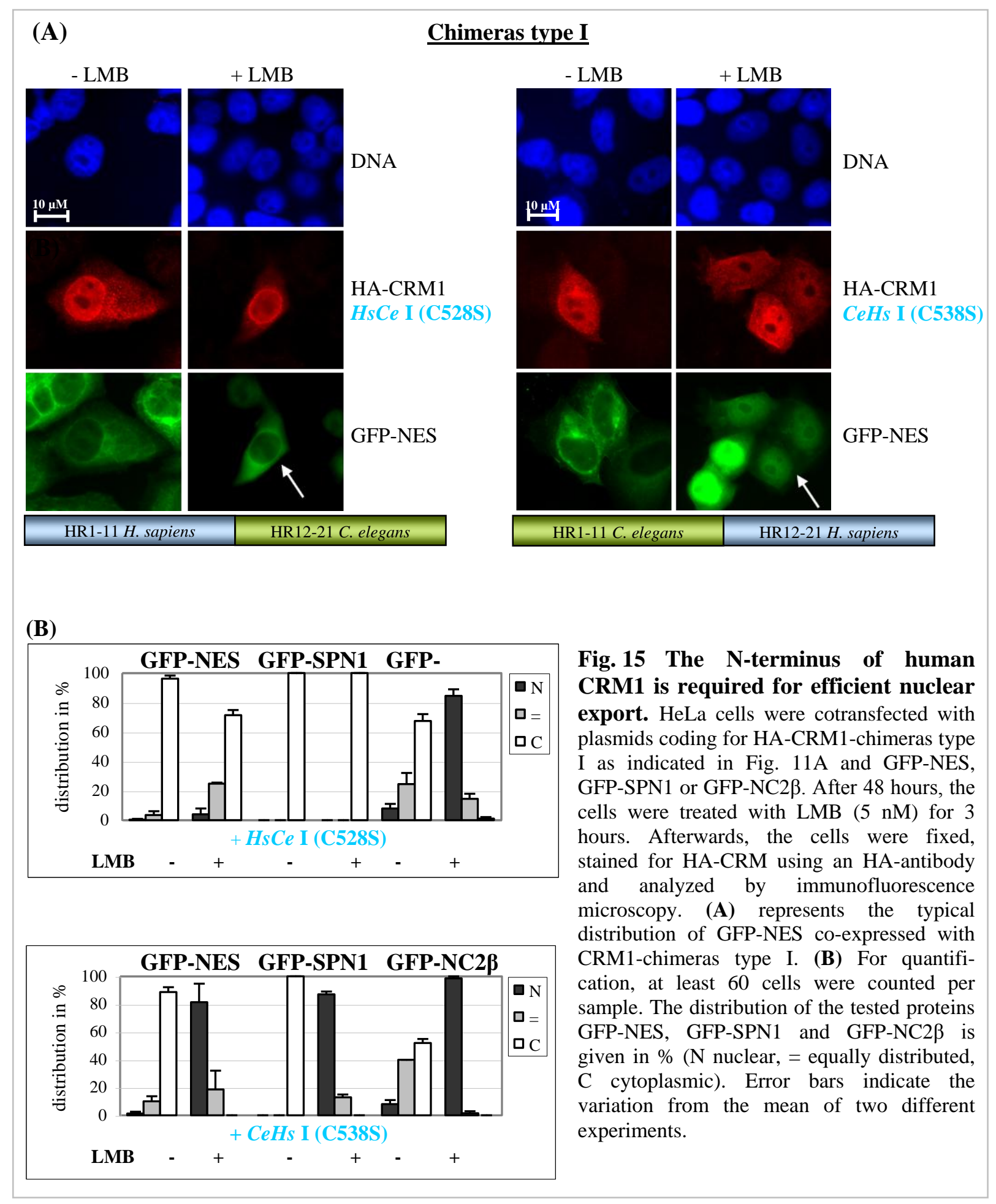


(A)
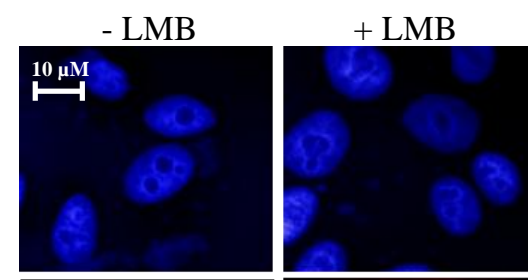

DNA
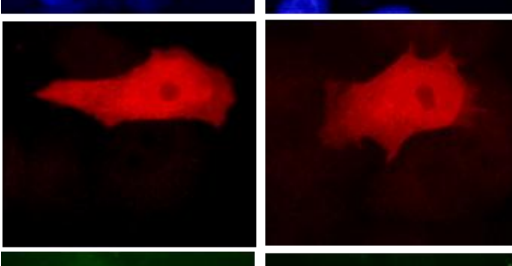

HA-CRM1

HsCe III (C538S)
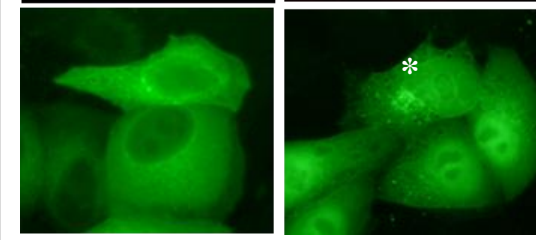

GFP-NES

HR 1-8 H. sapiens
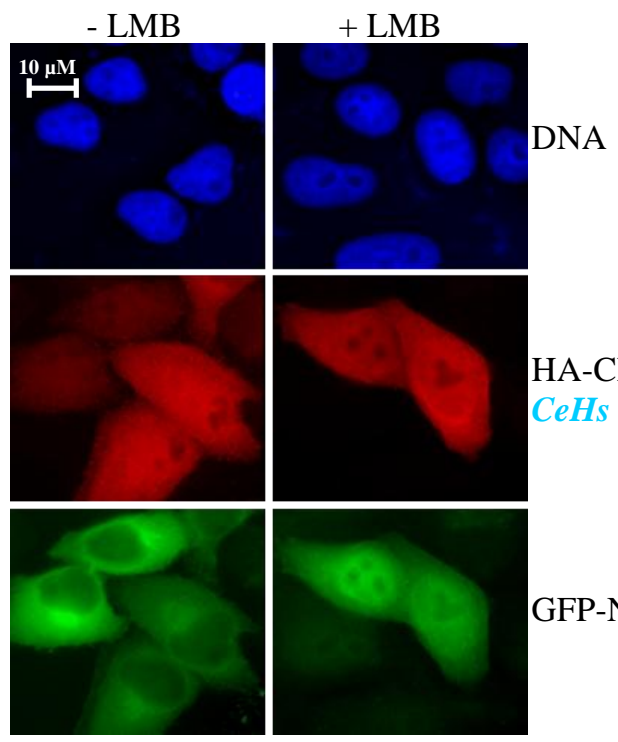

HR1-8 C. elegans

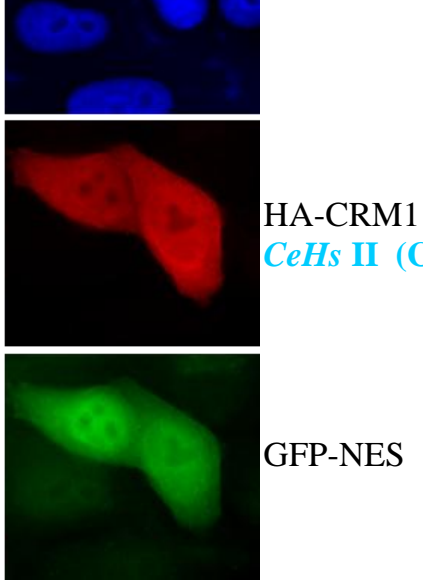

HR9-21 H. sapiens

(B)
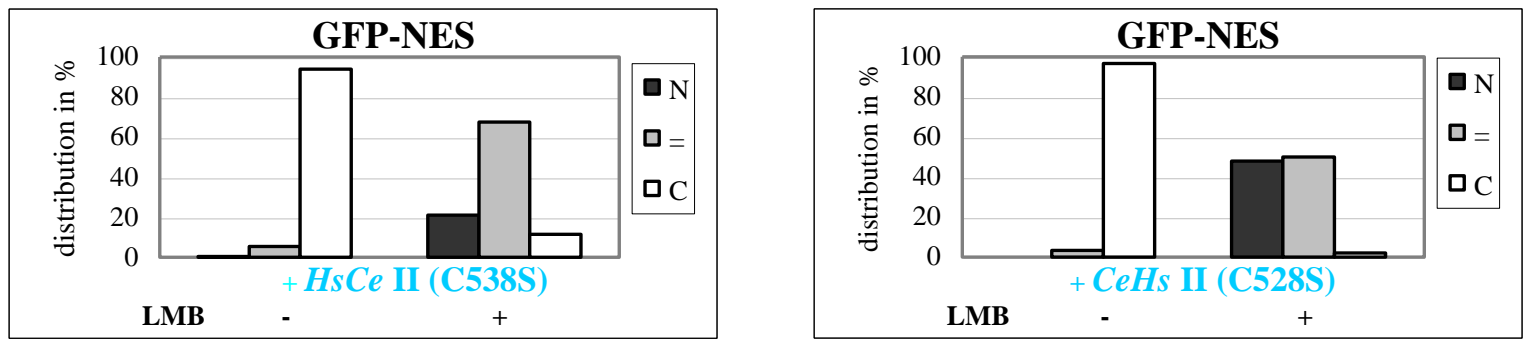

Fig. 16 CRM1-chimeras type II are restricted in their export capability. HeLa cells were cotransfected plasmids for CRM1-chimeras type II as indicated in Fig. 11A and GFP-NES. After 48 hours, the cells were treated with LMB $(5 \mathrm{nM})$ for 3 hours. Afterwards, the cells were fixed, stained for HA-CRM using an HA-antibody and analyzed by immunofluorescence microscopy. The pictures in (A) represent the typical distribution of GFP-NES co-expressed with CRM1-chimeras type II. (B) For quantification, at least 60 cells were counted per sample. The distribution of GFP-NES is given in \% (N nuclear, = equally distributed, $\mathrm{C}$ cytoplasmic). Error bars indicate the variation from the mean of two different experiments.

\section{CRM1}

\section{H. sapiens}

C. elegans

$\mathrm{HsCe}$ I (HR 1-11/12-21)

CeHs I (HR 1-11/12-21)

Localization Rim around the

Promoted nuclear export of

$\mathrm{HsCe}$ II (HR 1-8/9-21)

CeHs II (HR 1-8/9-21) nucleus

NES

SPN1 NC2 $\beta$

Table 2 Summary of analyzed CRM1-chimeras and export substrates. 


\subsubsection{The deletion of an outer surface loop between HEAT repeat $8 B$ and 9A of CRM1 does not affect nuclear export}

The differences in localization and functionality observed for the CRM1-chimeras led to the assumption that the responsible region for that is located in HR 9-11. The alignment of H. sapiens CRM1, C. elegans CRM1 and also D. melanogaster (Drosophila melanogaster) CRM1 revealed an unconserved sequence directly before HR 9 (Fig. 17, black frame). In the CRM1-structure, this sequence extends from the molecule as a flexible loop (Monecke et al., 2009).

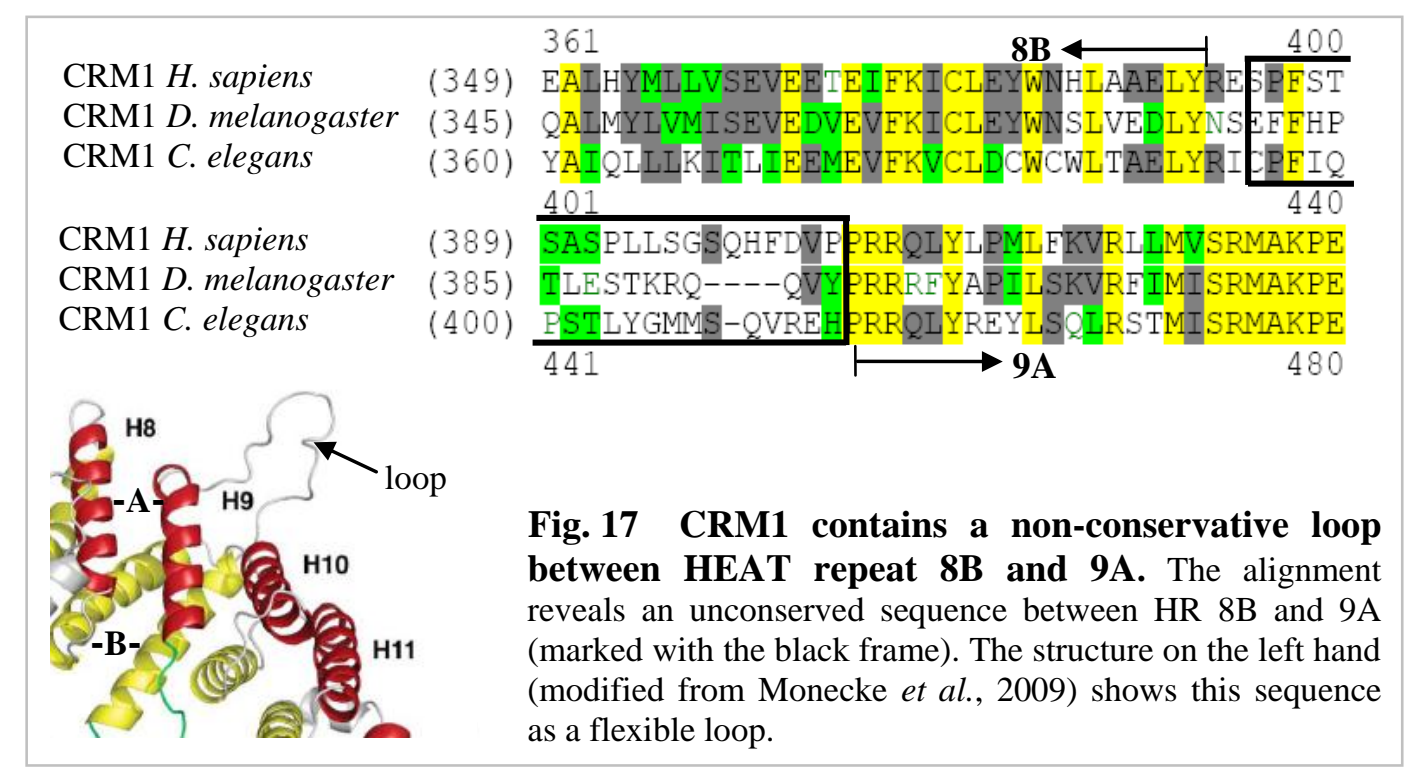

To investigate this region in CRM1, a mutant lacking aa 384-403 was constructed (containing the C528S mutation). A plasmid coding for this CRM1 mutant (named CRM1 C528S $\Delta$ loop) was transfected into HeLa cells and the location of GFP-NES and GFP-NC2 $\beta$ was analyzed in coexpressing cells after LMB treatment. For better comparison, the distribution of the GFP-proteins alone or in the presence of CRM1 C528S or CRM1 C528S $\triangle$ loop is summarized in Fig. 18B and C. Like WT CRM1, the C528S $\Delta$ loop mutant localized to the nucleus and at the nuclear envelope. GFPNES localization was cytoplasmic after LMB treatment (Fig. 18A), as it was observed for cells coexpressing CRM1 C528S. Moreover, overexpressed CRM1 C528S $\Delta$ loop promoted nuclear export of $\mathrm{NC} 2 \beta$ in untreated cells, as it has been described for CRM1 containing the loop (summarized in Fig. 18C, also compare Fig. 9B and 10B). Thus the loop between HR 8B and 9A is not required for export of proteins. 
(A)

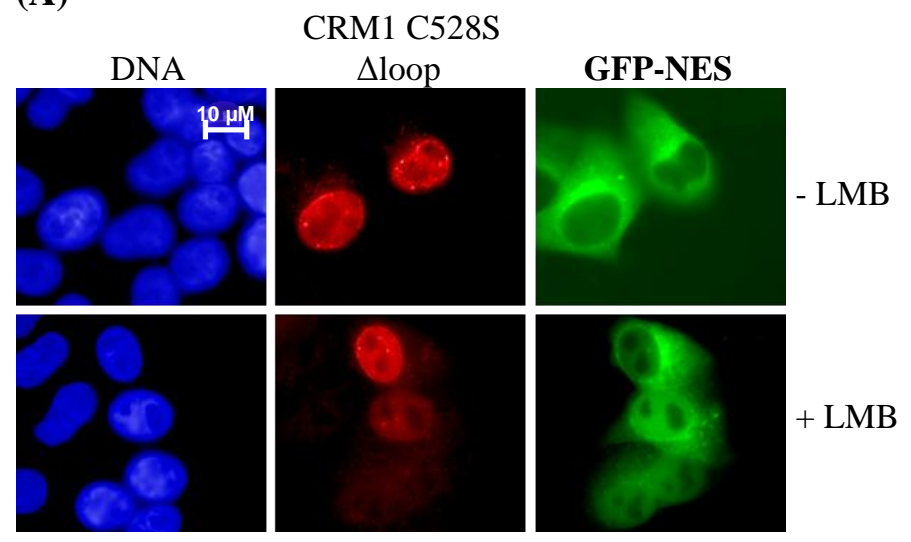

(B)

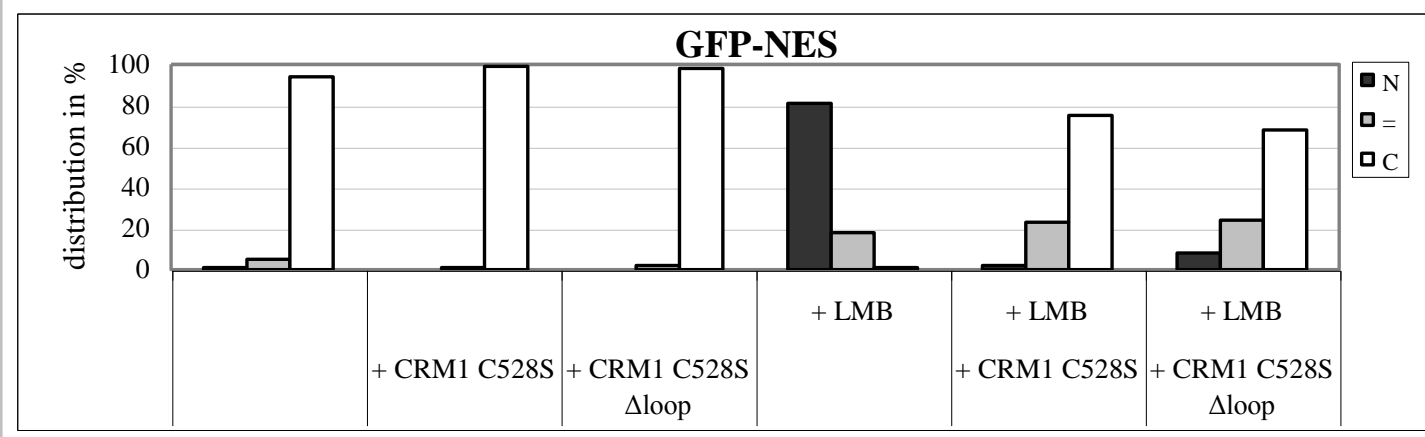

(C)

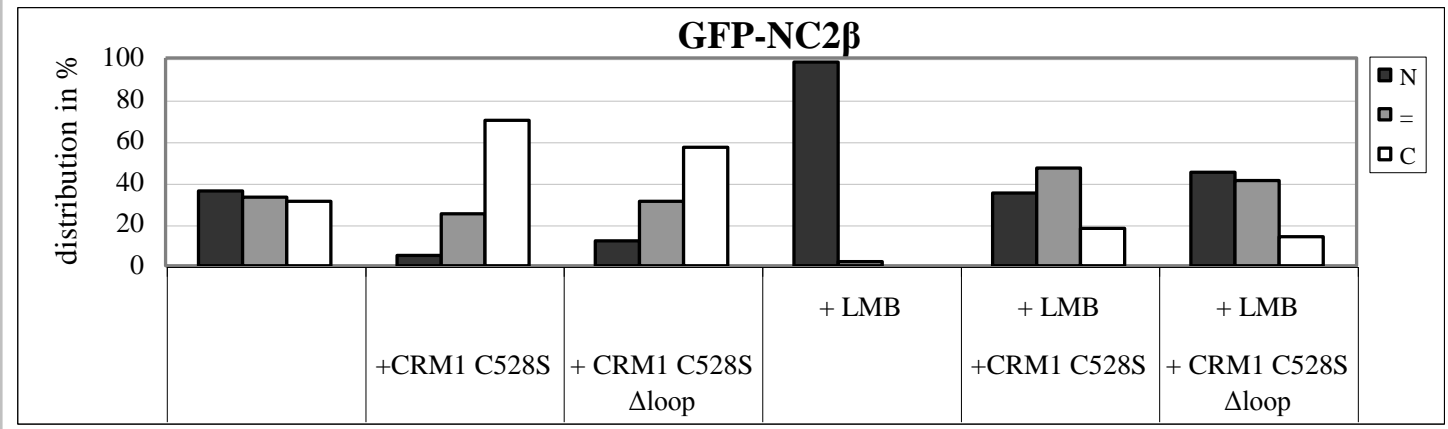

Fig. 18 CRM1 C528S $\Delta$ loop is able to export cargos out of the nucleus. HeLa cells were transfected with plasmids coding for HA-CRM1 C528S mutant or with HA-CRM1 C528S $\Delta$ loop together with GFP-NES (A, B) or GFP-NC2 $\beta$ (C). After 48 hours, the cells were treated with LMB $(5 \mathrm{nM})$ for 3 hours, fixed, stained for HA-CRM1 and analyzed by immunofluorescence microscopy. The distribution was quantified by counting $60-100$ cells per sample.

Regarding the data obtained from CRM1-chimeras and the loop deletion mutant, we assume that one or more regions in HR 9-11 in human CRM1 are required for interaction with nucleoporins and thus are also necessary for the function of CRM1 as an export receptor. These regions should differ between $H$. sapiens and C. elegans CRM1, as C. elegans CRM1 cannot interact with human nucleoporins. To prove this assumption, it will be required to create and analyze CRM1 with 
mutations in this region and more CRM1-chimeras with step-wise addition of human HEAT repeats in this region (e.g. chimeras $1-9 / 10-21$ or 1-10/11-21).

\subsection{Determination of a minimal region in Nup214 required for CRM1- binding}

Little is known about nucleoporin-CRM1 interaction in general. So far, the amino acid residues 1859-2090 of Nup214 have been identified to bind CRM1 in a RanGTP-dependent manner (Fornerod et al., 1996). Furthermore, the FG-repeats of Nup214 are assumed to be responsible for this binding, but so far there is no evidence for that. Here, we analyzed the binding of Nup214 aa 1859-2090 to CRM1 by using short Nup214-fragments and -mutants. To narrow down the Nup214-CRM1interaction site, in vivo analysis was combined with binding assays.

\subsubsection{Function of Nup214 C-terminal fragments in nuclear export}

Overexpression of Myc-Nup214 aa 1859-2090 cells led to apoptosis, growth defects, to nuclear accumulation of CRM1 and also to nuclear accumulation of Poly (A) ${ }^{+}$RNA (Boer et al., 1998). This led to the hypothesis that CRM1 accumulates in the nucleus because of its strong binding to C-terminal Nup214, and hence, would not be available for export cargos. We now set out to analyze the localization of other CRM1-dependent export cargos in cells overexpressing C-terminal MycNup214. HeLa cells were transfected with plasmids coding for GFP-NES, -NC2 $\beta$, -SPN1, -Nmd3 or -TFIIA $\alpha$ and additionally with a plasmid coding for C-terminal Myc-Nup214. After 24 hours, the cells were analyzed by immunofluorescence microscopy. Without C-terminal Myc-Nup214, most of the GFP-cargos were located in the cytoplasm (Fig. 19A). GFP-Nmd3 distributed equally between the nucleus and the cytoplasm. However, all tested cargos accumulated in the nucleus in the presence of C-terminal Nup214 (Fig. 19B). In cells weakly expressing Myc-Nup214 aa 1859-2090, cargos also distributed equally between the nucleus and the cytoplasm (see arrow).

Nuclear accumulation of the tested cargos further supports the assumption that endogenous CRM1 is held back in the nucleus in the presence of the exogenous Nup214 C-terminus. As a consequence, proteins that are imported remain in the nucleus. As this approach worked very well for the tested export cargos, it was also used for analysis of shorter Nup214-fragments to narrow down the Nup214CRM1-interaction site. 


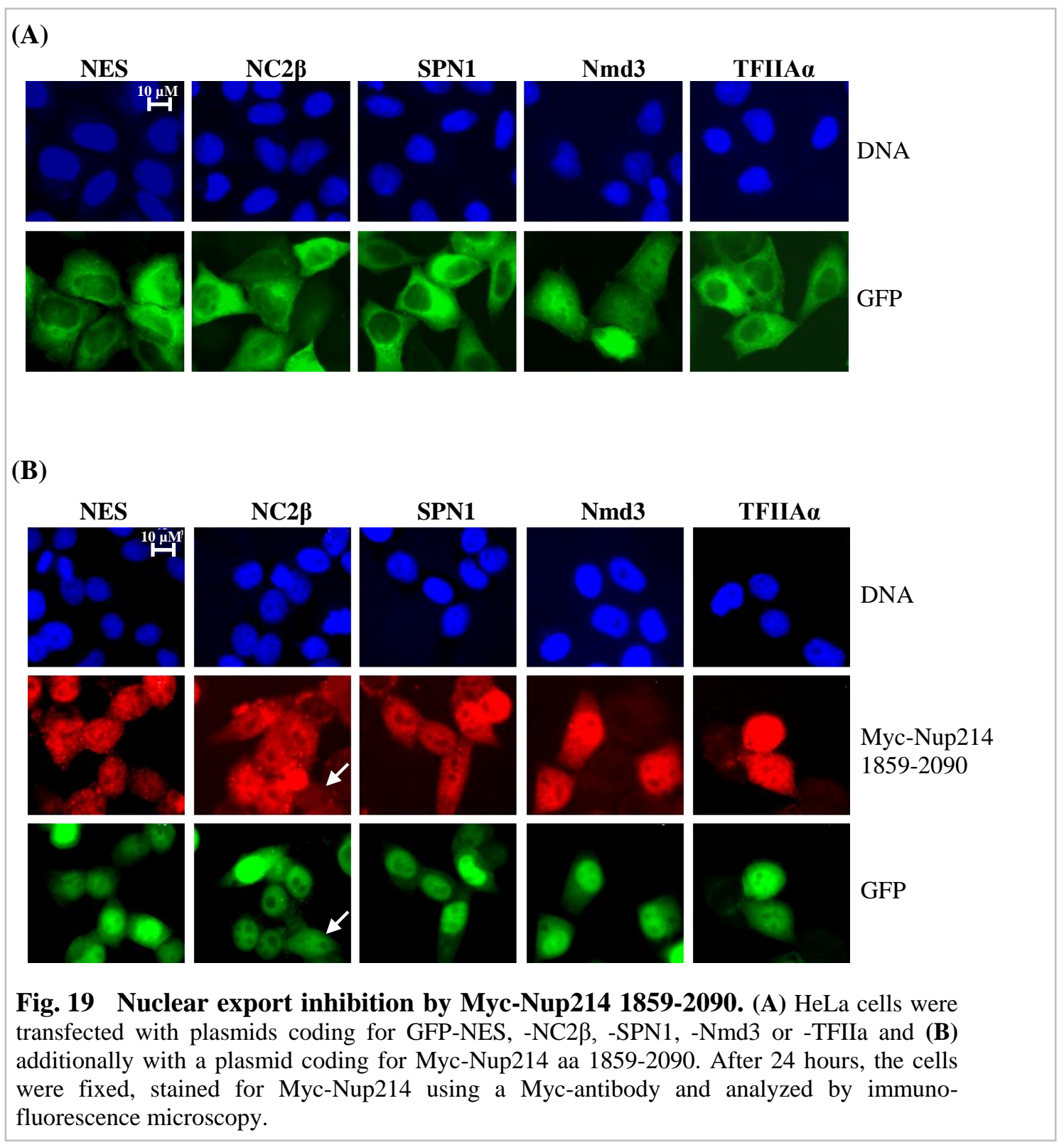




\subsubsection{Analysis of C-terminal Nup214-fragments in vivo}

To facilitate analysis, RFP-fused Nup214-fragments were constructed (Fig. 20A; for viewing all constructed fragments in this work, see appendix Table S1 and Fig. S2). Nup214 aa 1859-2090 accumulated in the nucleus, but shorter fragments could also reside in the cytoplasm. For this reason, a cNLS was added to the C-terminus of the fragments, leading to nuclear localization of all fragments. The RFP-Nup214-cNLS fragments or RFP-cNLS alone were co-expressed with GFP-SPN1 or GFPNC2 $\beta$ in HeLa cells. In the presence of RFP-cNLS, GFP-SPN1 and GFP-NC2 $\beta$ were detected in the cytoplasm, demonstrating that RFP-cNLS alone does not influence nuclear export of these cargos (Fig. 20B and C). By contrast, co-expression of RFP-Nup214 (1859-2090)-cNLS fragment (fragment 1, compare Fig. 20A) led to a nuclear accumulation of the GFP-cargos. For GFP-NC2 $\beta$, the coexpression with this Nup214-fragment had a somewhat milder effect. In 70\% of the cells, GFP-NC2 $\beta$ was nuclear, and in $30 \%$ of the cells, the cargo was distributed equally between the nucleus and the cytoplasm (Fig. 20C and E). Basically, expression of this RFP-Nup214-cNLS fragment led to nuclear accumulation of CRM1-dependent export cargos as it was previously observed for the corresponding Myc-Nup214-fragment. Using this approach, we now set out to analyze shorter Nup214-fragments. Surprisingly, the presence of RFP-Nup214 (1859-1974)-cNLS fragment (fragment 2) did not affect the localization of GFP-SPN1 (Fig. 20B and D), whereas GFP-NC2 $\beta$ was located nuclear or distributed equally between the nucleus and the cytoplasm (Fig. 20C and E). This indicates that NC2 $\beta$ might react in a more sensitive manner to overexpression of Nup214-fragments. Co-expression of the other Nup214-fragments aa 1975-2090 and 1916-2033 (fragment 3 and 4) led to a nuclear accumulation of both cargos. Concluding from that, one important Nup214 region, responsible for nuclear accumulation of export cargos, is located between amino acid residue 1975 and 2033. However, it cannot be excluded that there are other regions in Nup214 supporting or regulating CRM1- mediated nuclear export.

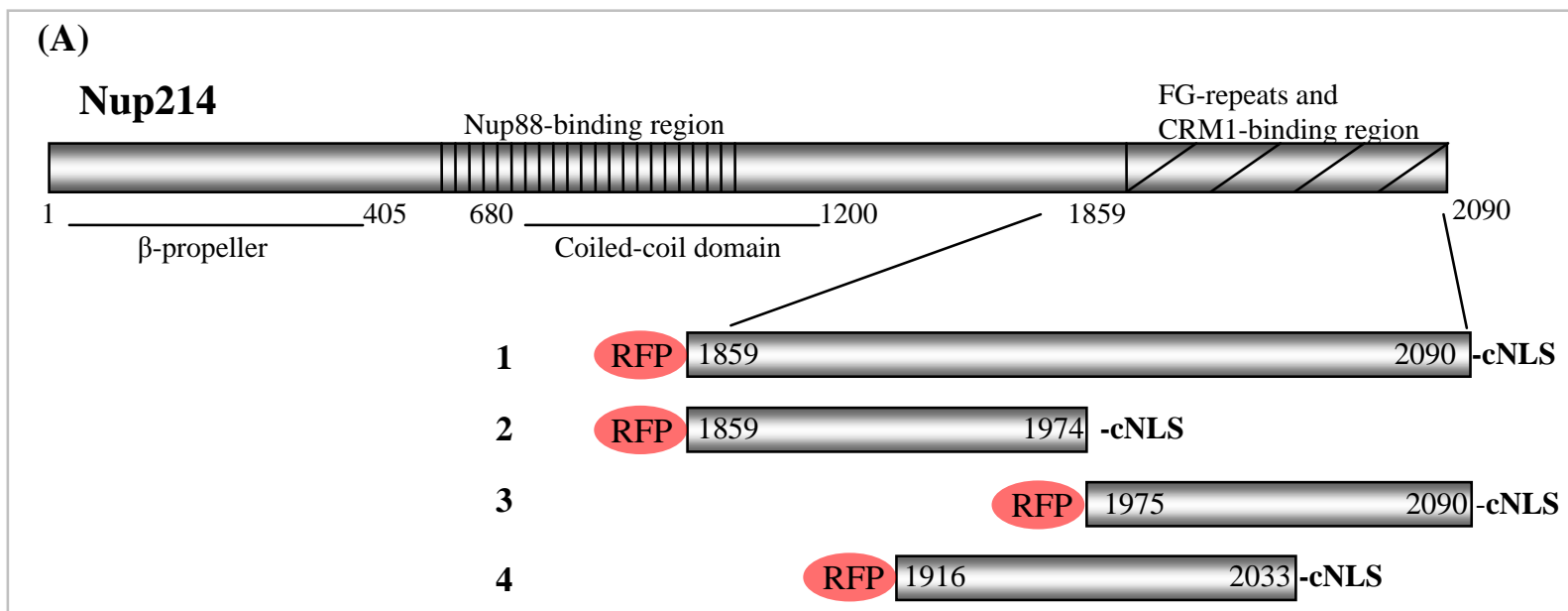

Fig. 20 (A) Nup214-fragments containing amino acids 1975-2033 inhibit nuclear export of proteins. Schematic view of the Nup214 C-terminal fragments. The fragment aa 1859-2090 had been identified as the CRM1-binding region in vitro (Fornerod et al., 1996). 


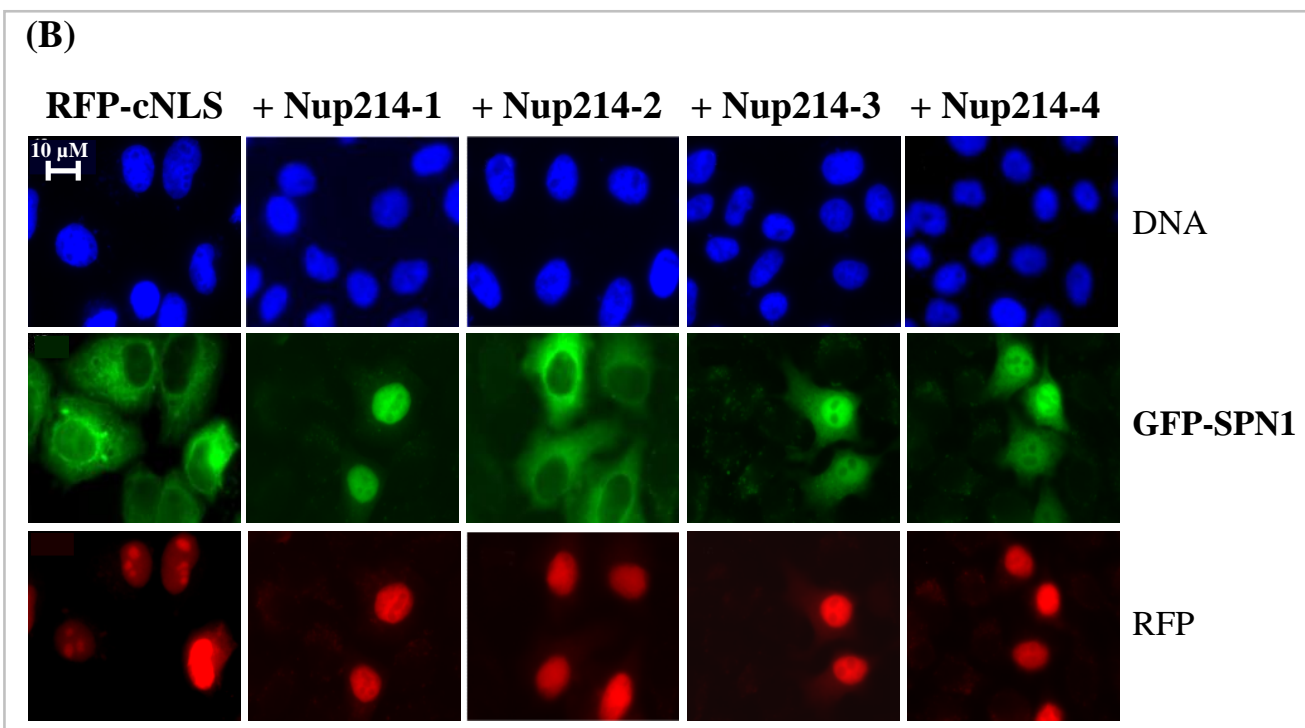

(C)
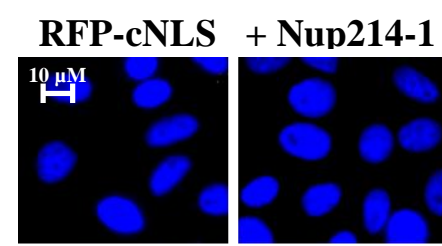

+ Nup214-2

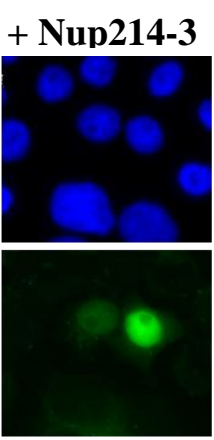

+ Nup214-4
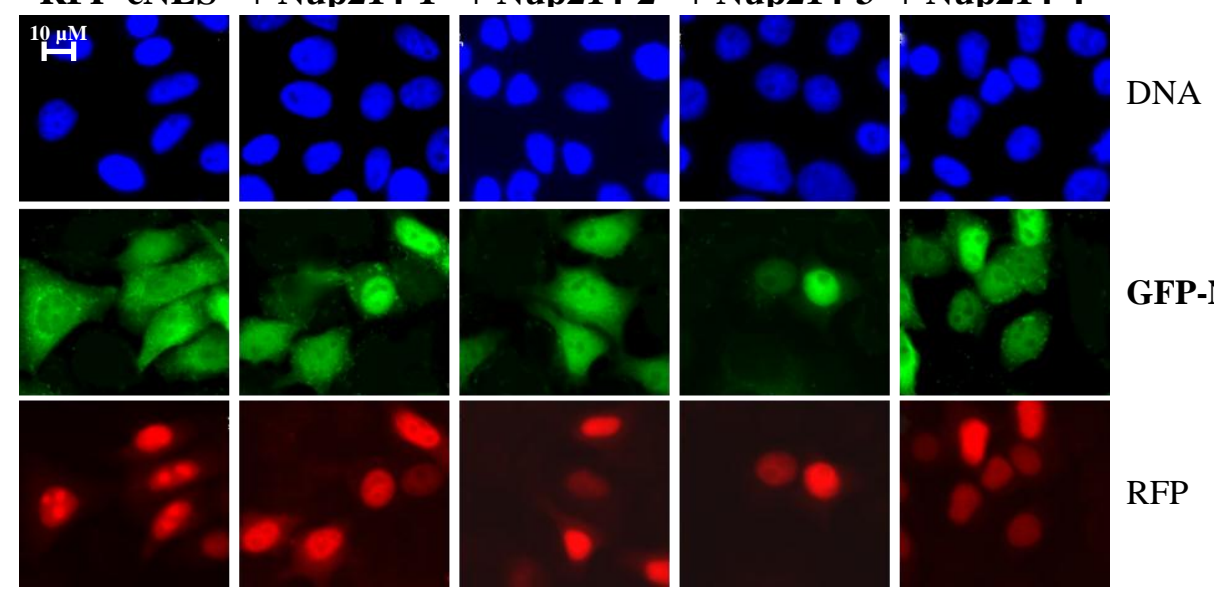

GFP-NC2 $\beta$

(D)

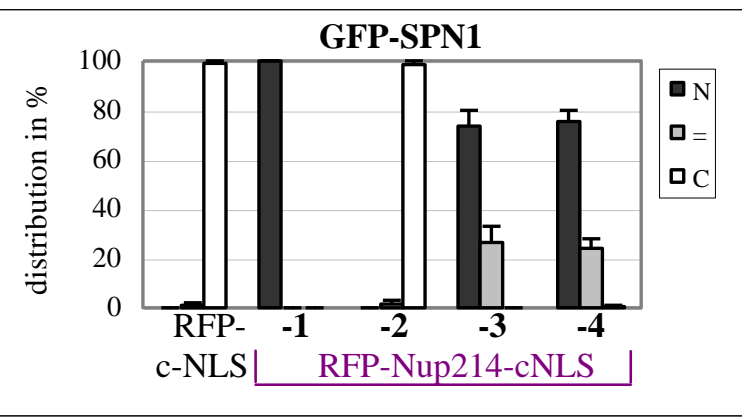

(E)

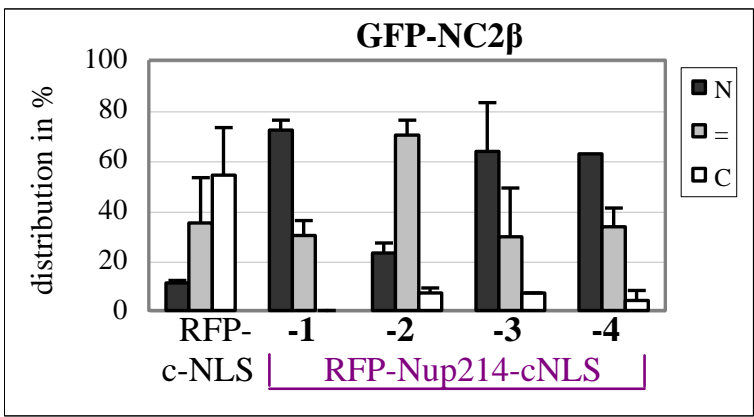

Fig. 20 (B-E) Nup214-fragments containing amino acids 1975-2033 inhibit nuclear export of proteins. Plasmids coding for Nup214-fragments as depicted in (A) were cotransfected with GFP-SPN1 (B) or GFP-NC2 $\beta$ (C) in HeLa cells. After 20 hours, the cells were fixed and analyzed by immunofluorescence microscopy. The distribution (in \%; N nuclear, = equally distributed, C cytoplasmic) of GFP-SPN1 (D) and GFP-NC2 $\beta$ (E) co-expressing RFP-Nup214 fragments was quantified by counting at least 100 cells. Error bars indicate the variation from the mean of two independent experiments. 


\subsubsection{Accumulation of CRM1 in the nucleus upon overexpression of Nup214-fragments}

Overexpression of Nup214 aa 1859-2090 inhibits CRM1-dependent nuclear export and also leads to nuclear accumulation of CRM1 (Boer et al., 1998). Based on that, shorter Nup214-fragments that inhibit nuclear export should also lead to accumulation of CRM1 in the nucleus. In order to test this, HeLa cells were transfected with Nup214-fragments as indicated in Fig. 20A. Except of RFP-Nup214 (1859-1974)-cNLS (fragment 2), all other fragments led to enrichment of CRM1 in the nucleus (Fig. 21). Cells overexpressing Nup214-fragments 1, 3 and 4 were often smaller than untransfected cells and also occurred pairwise. This indicates an arrest of the cells in the $\mathrm{G}_{0}$ phase and reduced cell growth as it has been described for Myc-Nup214 aa 1859-2090 (Boer et al., 1998).

These results show that the inhibitory effect of Nup214-fragments on nuclear export correlates with retention of CRM1 in the nucleus.

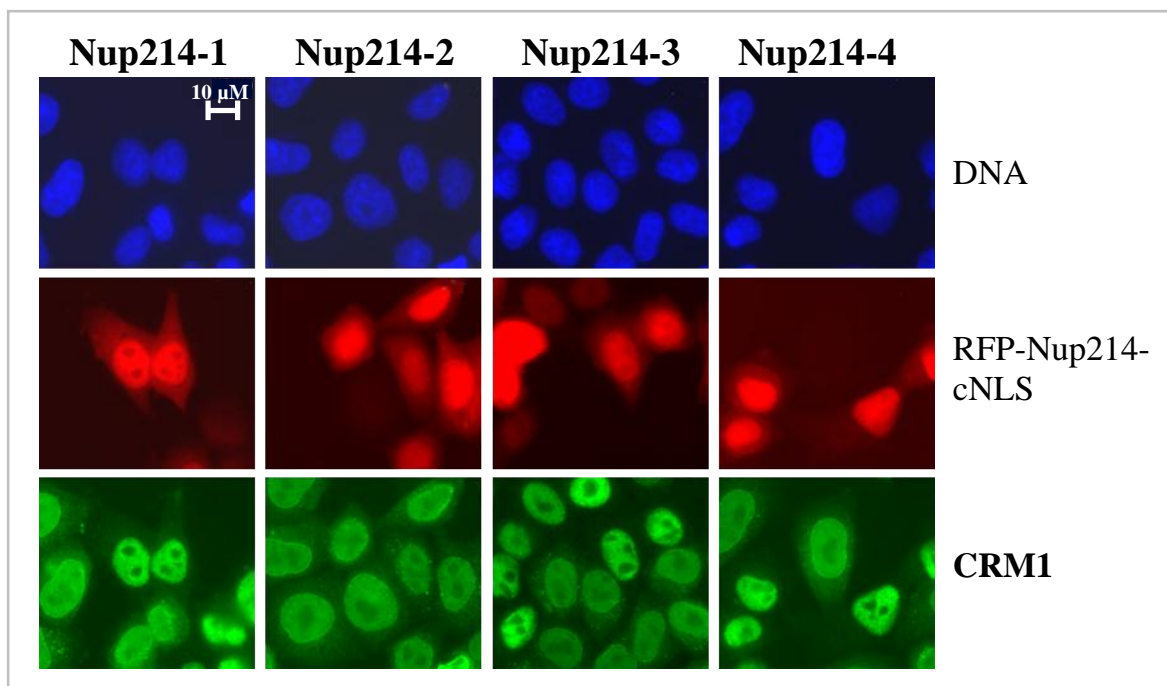

Fig. 21 Nuclear accumulation of CRM1 in the presence of export inhibiting Nup214 fragments. HeLa cells were transfected with plasmids coding for RFP-Nup214-fragments as indicated in Fig 22A. After 20 hours, cells were fixed, stained for endogenous CRM1 and analyzed by immunofluorescence microscopy. 


\subsubsection{Nup214 1916-2033 shows an inhibitory effect on CRM1-dependent nuclear export of}

\section{$\underline{G F P-N F A T \text { in vitro }}$}

Formally, the observed accumulation of export cargos in HeLa cells could be due to altered nuclear import. For this reason, a Nup214-fragment was bacterially expressed and its effects were tested in an in vitro system using digitonin-permeabilized HeLa cells stably expressing GFP-NFAT (Kehlenbach et al., 1998). The transport of the shuttling transcription factor GFP-NFAT (nuclear factor of activated T-cells) is regulated by phosphorylation/dephosphorylation. NFAT contains two cNLSs (Beals et al., 1997a) and a NES, recognized by CRM1 (Kehlenbach et al., 1998). Treatment of GFP-NFAT cells with the calcium ionophore ionomycin leads to dephosphorylation of the cNLS by calcineurin. Hence, NFAT becomes available for the importin $\alpha / \beta$ complex and is imported into the nucleus. Once inside the nucleus, the glycogen synthase kinase 3 (GSK-3) phosphorylates the cNLS and NFAT is exported into the cytoplasm (Beals et al., 1997b). For the assay, nuclear import of GFP-NFAT was induced and the cells were permeabilized with digitonin, allowing permeabilization of the cell membrane while the nuclear membrane is kept intact. After washing out the cytosol, external transport factors were added. As a control, the cells were additionally incubated with the import substrate BSA-Cy5-cNLS. Hence, it is possible to analyze import and export in the same cell. The transport reaction was started by shifting the temperature of the samples to $30^{\circ} \mathrm{C}$. Afterwards, nuclear fluorescence of BSA-Cy5-cNLS and GFP-NFAT was analyzed by flow cytometry. Nuclear fluorescence of BSA-Cy5-cNLS (Fig. 22A) increased in the presence of cytosol and Ran (mean $=0.43$ ) compared to the buffer reaction (mean = 0.26). Thus, nuclear import of BSA-Cy5-cNLS could take place under these conditions. In cells treated with buffer, nuclear fluorescence of GFP-NFAT was very high (Fig. 22B; mean = 7.2). Treatment of the cells with cytosol led to a decrease in nuclear fluorescence (mean $=4.2$ ), which was further decreased by additional Ran (mean $=1.5$ ). With this, nuclear export of GFP NFAT was clearly stimulated compared to the buffer reaction. The cells were also treated with wheat germ agglutinin (WGA) which blocks the nuclear pore (Finlay et al., 1987). The high nuclear fluorescence of GFPNFAT indicates that the nuclear envelope of the permeabilized cells was intact. Nuclear fluorescence of GFP-NFAT was also high after incubation of the cells with all transport factors at $4{ }^{\circ} \mathrm{C}$, showing the specificity of the transport reaction at $30{ }^{\circ} \mathrm{C}$. The nuclear fluorescence of GFP-NFAT, or BSA-Cy5cNLS, respectively, was unaltered by increasing amounts of GST. However, increasing concentrations of His-Nup214 (aa 1916-2033) led to increasing nuclear fluorescence of GFP-NFAT (see red frame). In contrast to that, nuclear fluorescence of Cy5-BSA-cNLS was unaffected in the presence of the Nup214-fragment. Together, Nup214 aa 1916-2033 inhibits nuclear export, and does not affect nuclear import. 
(A)

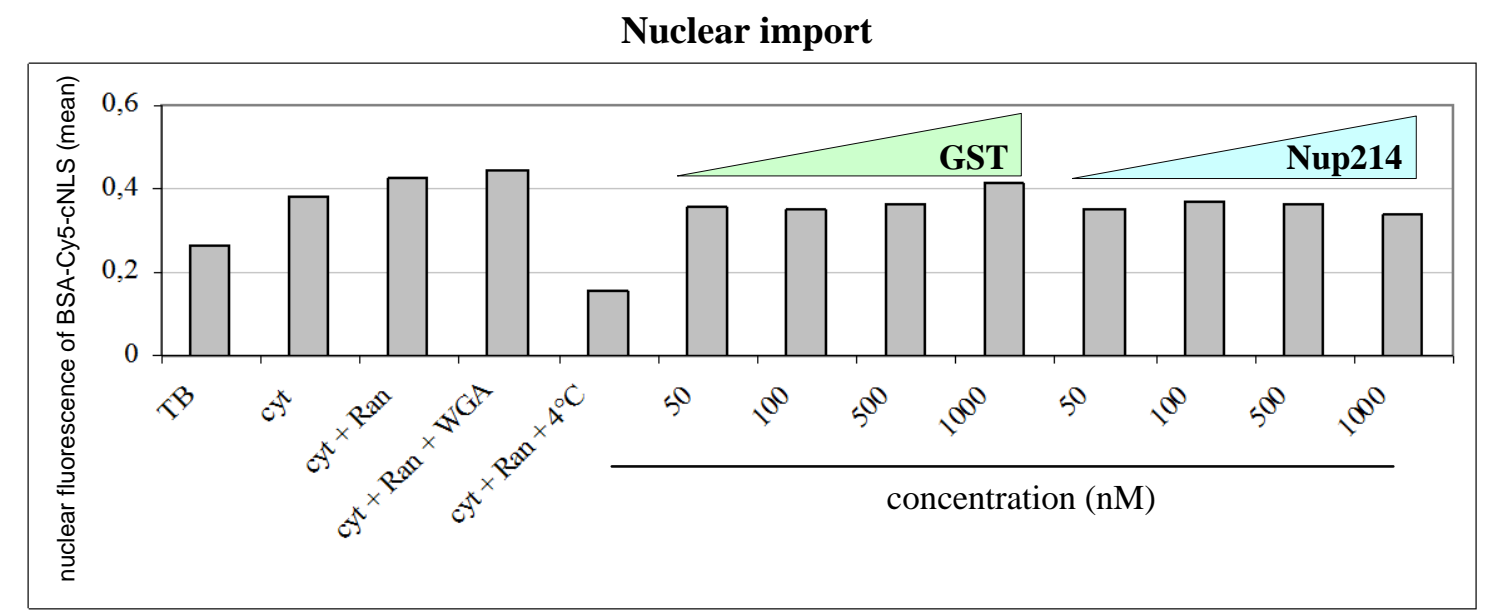

(B)

\section{Nuclear export}

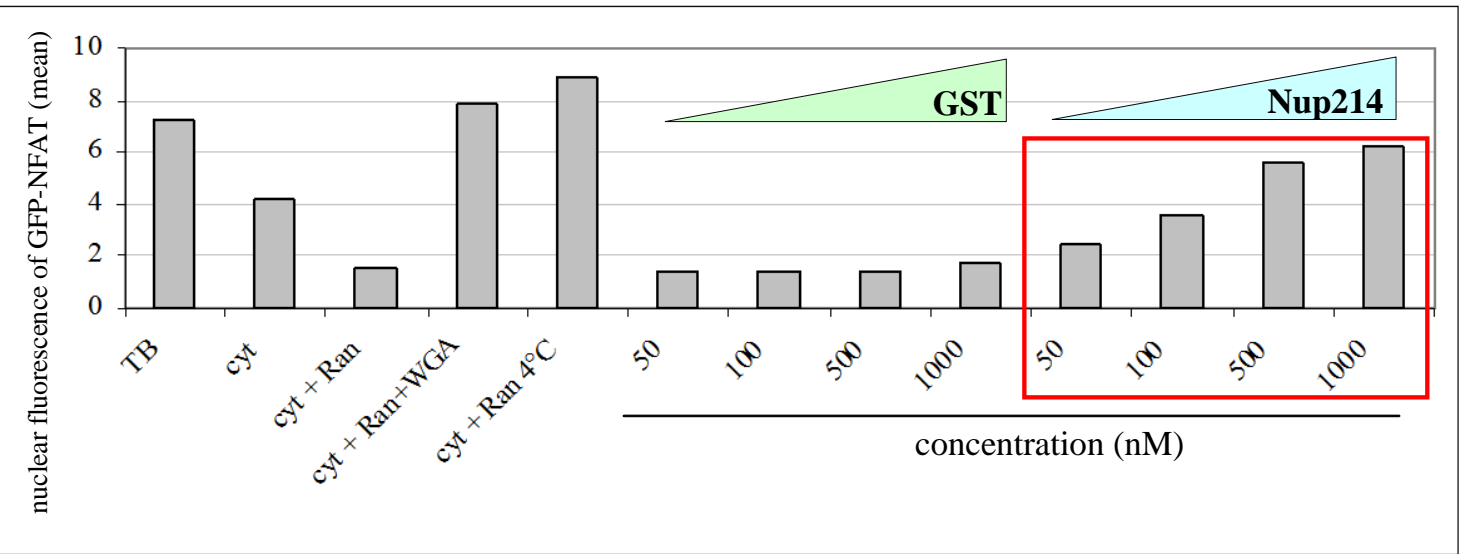

Fig. 22 Inhibition of nuclear export of GFP-NFAT by His-Nup214 aa 1916-2033 in vitro. Stable GFP-NFAT HeLa cells were permeabilized with digitonin and subjected to export reactions in vitro. BSA-Cy5-cNLS and cytosol, Ran and different concentrations of GST (control) or His-Nup214 aa 1916-2033 were added as indicated. The nuclear fluorescence of BSA-Cy5-cNLS (A) and GFP-NFAT (B) was analyzed by flow cytometry.

We also analyzed the localization of CRM1 and Ran in the presence of recombinant Nup214 in adherent cells. The cells were permeabilized with digitonin, washed and supplied with cytosol, Ran, an ATP-regenerating system, and additionally with Nup214 aa 1916-2033. After incubation at $30^{\circ} \mathrm{C}$, the cells were analyzed by immunofluorescence microscopy (Fig. 23). Cells treated with cytosol, Ran and ATP hardly showed any staining for CRM1 or Ran, whereas in cells incubated with additional His-Nup214-fragment, CRM1 and Ran became clearly nuclear. This suggests that CRM1 was bound to His-Nup214 in a complex with Ran. 
Taken together, Nup214 aa 1916-2033 leads to accumulation of CRM1 and Ran in HeLa cells and therefore inhibits nuclear export of CRM1-dependent cargos. This supports the idea that there is a CRM1-binding region within Nup214 aa 1916-2033.

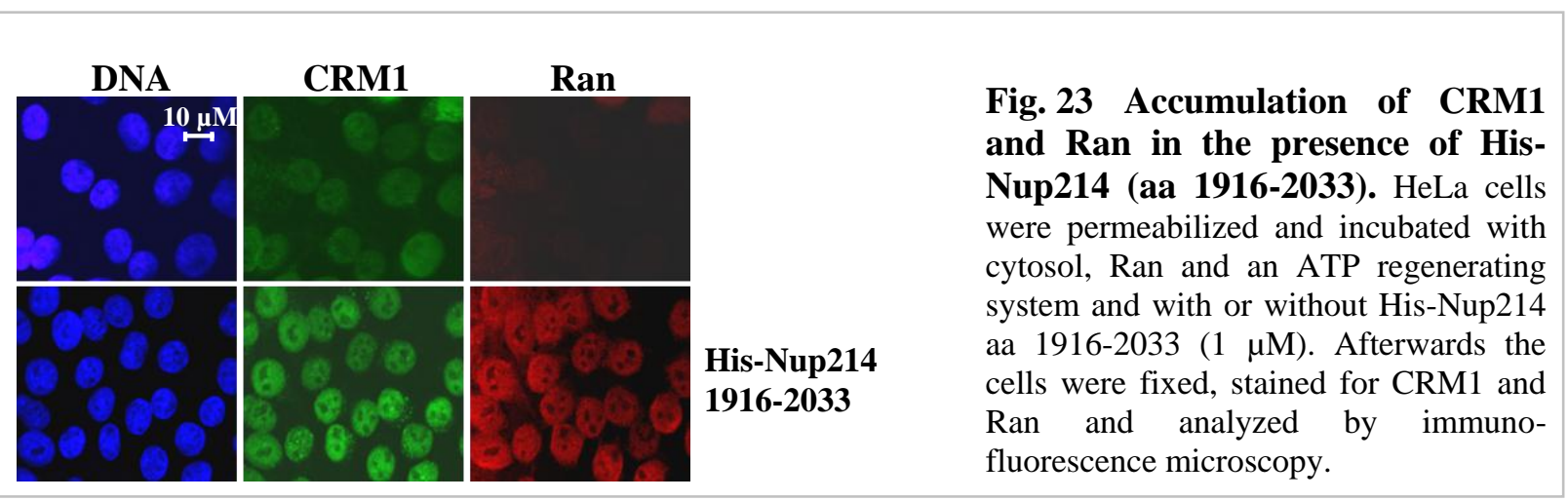

\subsubsection{Analysis of a highly conserved FG-motif in Nup214}

The investigation of Nup214-fragments in HeLa cells indicated that in the C-terminal part aa 18592090 some regions are required for nuclear export inhibition and nuclear retention of CRM1, whereas others are not. Based on the previous results, we hypothesized that Nup214-fragments which lead to nuclear retention of CRM1 and CRM1-dependent cargo proteins in vivo would also bind directly to CRM1 in vitro. To analyze putative CRM1-binding regions in Nup214, we combined functional assays with binding studies by using short Nup214-fragments and -mutants.

\subsubsection{Nup214 aa 1916-2033 contains a CRM1-binding site}

CRM1 is known to bind together with RanGTP and a NES-cargo as a trimeric export complex to Nup214 aa 1859-2090 (Fornerod et al., 1996; Kehlenbach et al., 1999). The binding of shorter Nup214-fragments was now investigated in pull down assays. His-Nup214 C-terminal fragments, corresponding to the tested RFP-cNLS fragments, were constructed and purified (Fig. 24A). HisNup214 aa 1975-2090 was also expressed in bacteria, but unfortunately it was insoluble.

GST-Ran either loaded with GDP or GTP was used as a bait. His-CRM1, an NES peptide (Askjaer et $a l ., 1999)$ and the Nup214-fragments were added and complex formation was analyzed by Western blot. Strikingly, Nup214 (aa 1859-1974) did not bind to CRM1, whereas Nup214 (aa 1916-2033) did (Fig. 24B). Furthermore, binding of CRM1 to the latter was increased in the presence of RanGTP, which is consistent with data obtained from C-terminal Nup214, which had been identified as the CRM1-binding site (aa 1859-2090; Fornerod et al., 1996). Interestingly, binding ability of Nup214 to 
CRM1 correlated with nuclear export inhibition and CRM1-accumulation in the nucleus (compare Fig. 21). From this we conclude that endogenous CRM1 accumulates in the nucleus in mammalian cells due to direct binding to overexpressed Nup214-fragments.
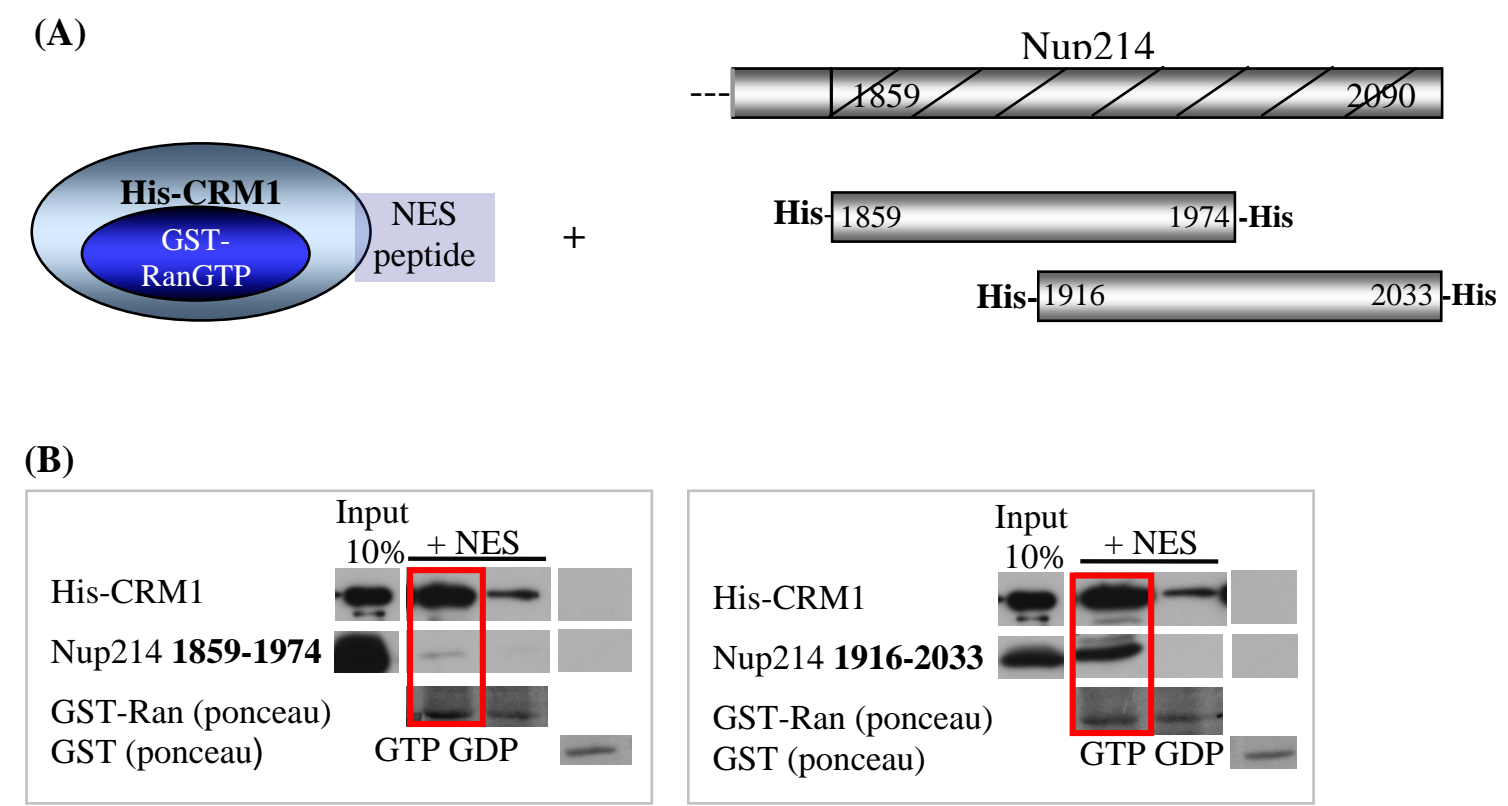

Fig. 24 Nup214-fragments differ in their CRM1-binding capability. (A) Schematic view of His-tagged Nup214-fragments and an illustration of the performed binding study in (B). GST $(2.5 \mu \mathrm{g})$, GST-RanGDP or GST-RanGTP $(5 \mu \mathrm{g})$ was immobilized on glutathione sepharose beads and incubated with His-CRM1 $(5 \mu \mathrm{g})$ and an NES peptide $(2500 \mathrm{nM}$, Askjaer et al., 1999). To the trimeric export complex, His-Nup214-fragments $(1 \mu \mathrm{g})$ aa 1859-1974 (left panel) or aa 1916-2033 (right panel) were added, respectively. CRM1 and Nup214 were detected by Western blot using anti-His-antibodies.

\subsubsection{The mutation of the FGs in the FG-motif in Nup214 aa 1916-2033 leads to severe CRM1-binding defects}

To narrow down the CRM1-binding site in Nup214 aa 1916-2033, we searched for prominent regions in the C-terminal region which might contribute to CRM1-binding. An alignment of the amino acid sequence of Nup214 revealed a highly conserved region (Fig. 25A). It consists of four phenylalanineglycines (FGs) with an identical distance from each other, and four prolines with a defined interval between the FGs. It is still not known in which way CRM1 binds to Nup214, but it is assumed that the binding occurs via the FGs, as it was observed for other transport receptors like importin $\beta$ (Bayliss et al., 2002). Based on this, mutants of Nup214 aa 1916-2033 (Fig. 25B) were analyzed with respect to their binding capabilities to CRM1. In Nup214 mutant a1, the four FGs in the highly conserved region are replaced by serin-glycines (SGs). Mutant a2 contains alanines instead of prolines in this region. In mutant a3 all FGs in the whole amino acid sequence 1916-2033 are replaced by SGs. 


\begin{tabular}{|c|c|}
\hline (A) & \\
\hline & 1965 \\
\hline B. taurus & NKTGGFG AAP VFG S P P T FGG S PGFGGVPAF \\
\hline H. sapiens & NKTGGFG AAP VFG S P P T FGG S PGFGGVPAF \\
\hline C. elegans & - TS SVFG GA P KFGS QP A FGAK P - FGGGANA \\
\hline D. melanogaster & T TPGGFG AK P VFGG S P A FGA S PT FGGGATF \\
\hline X. laevis & TKTGGFG AA P VFG S P P T FGG S PG FGGS PAF \\
\hline M. musculus & NKTGGFG AAP VFG S PP T FGG S PG FGGVPAF \\
\hline R. norwegicus & NKTGGFG AAP VFG S PP T FGG S PG FGGVPAF \\
\hline & FG x x PxFG x x P x FG x x Px FG xx Px \\
\hline
\end{tabular}

(B)

WT

a1

a2

a3
FG $x x$ P $x$ FGxx $P$ x FG $x$ x $P$ x FGxx $P$ x

SG $x x$ P $x$ SG $x x$ P $x$ SG $x x$ P $x$ SG $x x$ P $x$

FG xx A x FGxx A x FGxx A x FGxx A x

all FGs in aa 1916-2033 mutated to SG
Fig. 25 Nup214 contains a prominent

FG-motif. (A) The Nup214-alignment of several species revealed a highly conserved region between aa 1968 and 1990 consisting of a very prominent FG-motif. (B) His-Nup214 mutants were constructed and named a1, a2 and a3 mutants.

Note that in Western blot analysis, the Nup214 a1 mutant appears in two bands (see asterisk in Fig. 26A) and runs approximately $2-3 \mathrm{kDa}$ higher in SDS-PAGE than the WT protein (data not shown). The Nup214 a3 mutant also shows a similar running behaviour like the a1 mutant and is, moreover, not detectable in Western blots using our anti-Nup214 antibody (due to a FG mutation in the epitope). For these reasons, the proteins were analyzed by mass-spectrometry (kindly performed by Olaf Bernhardt, AG Dr. Bernhard Schmidt, Biochemistry II, Göttingen). In all four cases (WT, a1, a2, a3), Nup214 could be detected, confirming the identity of the expressed proteins (data not shown). With His-Nup214 aa 1916-2033 WT and the mutants, a pulldown assay was performed as described before. His-SPN1 was used instead of an NES-peptide as an export cargo. Whereas Nup214 WT bound to the trimeric export complex, neither the a1 nor the a3 mutant was able to bind CRM1 (Fig. 26). The exchange of the prolines to alanines (a2 mutant) did not affect CRM1-binding. 


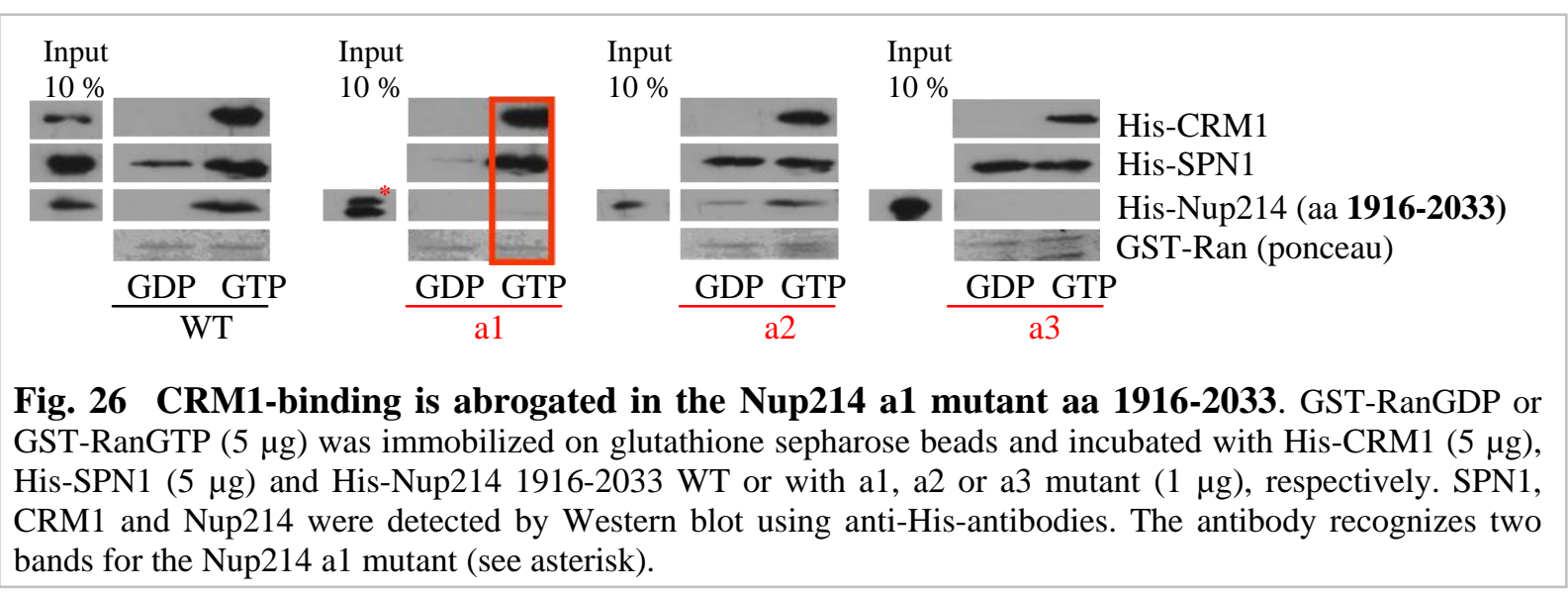

As His-Nup214 aa 1916-2033 a1 and a3 mutant were not able to bind CRM1 in vitro, we asked whether the localization of endogenous CRM1 would change in cells overexpressing the Nup214 mutants. To address this question, the a1, a2 and a3 mutations were introduced into RFP-Nup214 (aa 1916-2033)-cNLS. The mutants were overexpressed in HeLa cells and the localization of endogenous CRM1 was analyzed (Fig. 27). In the presence of Nup214 aa 1916-2033 WT, CRM1 accumulated in the nucleus, as it was previously observed (Fig. 21, fragment 4). The a2 mutant also retained CRM1 in the nucleus, indicating that the prolines in the FG-motif are not required for nuclear accumulation of CRM1. Surprisingly, in the presence of Nup214 a1 mutant CRM1 accumulated in the nucleus to a certain degree (see arrow), though this accumulation was not as strong as observed for WT Nup214. This suggests that not only these 4 FGs are involved in CRM1 nuclear accumulation. With respect to CRM1-binding, there might be other regions in Nup214 aa 1916-2033. This idea was further supported by the observation that the mutation of all FGs in Nup214 aa 1916-2033 (mutant a3) abolished nuclear retention of CRM1. It can be excluded that FGs per se are responsible for nuclear accumulation of CRM1, as Nup214 1859-1974, a fragment that contains eight FGs, did not affect CRM1 localization (see Fig 21, fragment 2). From these data, we concluded that the 4 FGs in the conserved region are essential for CRM1 interaction with Nup214 aa 1916-2033 in vitro. 


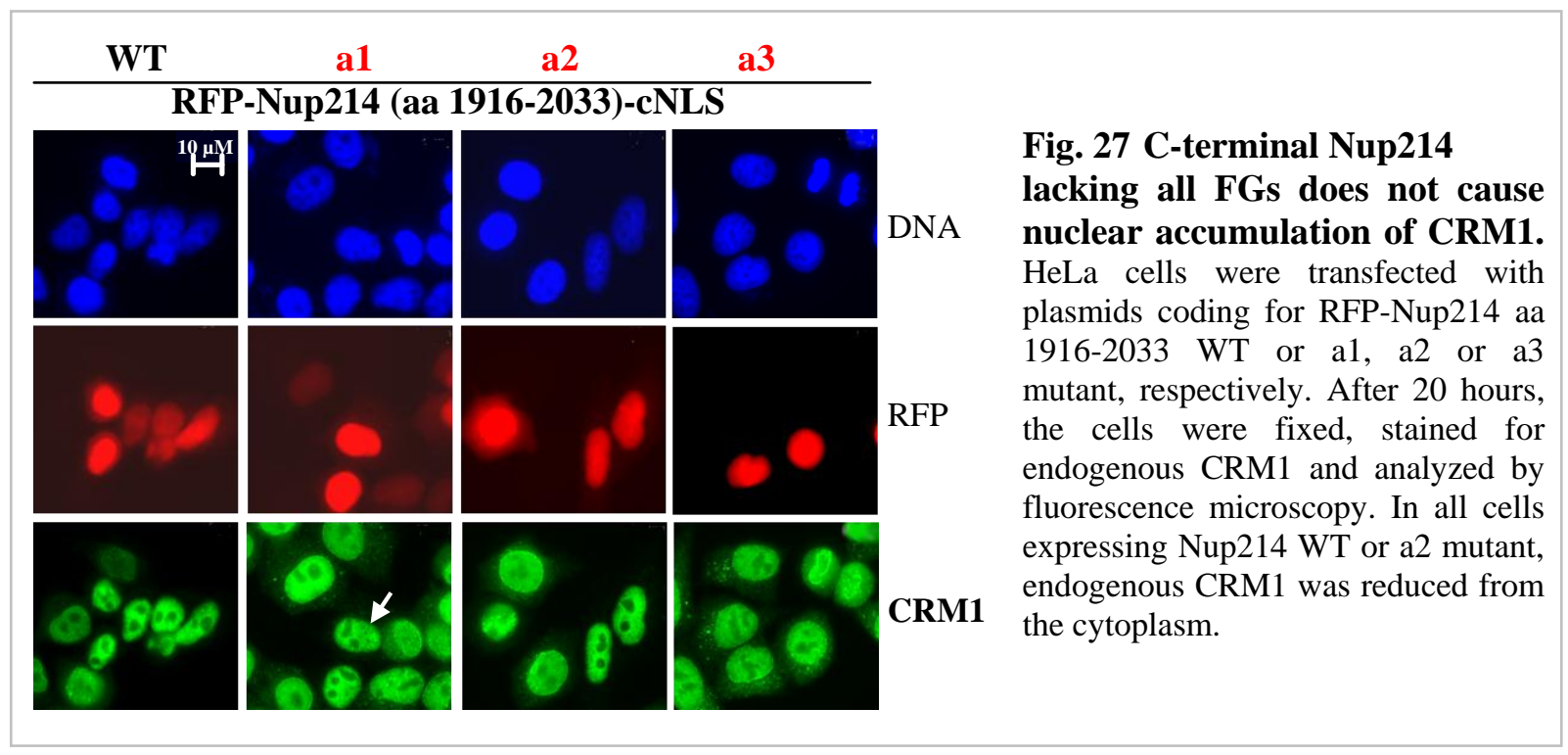

\subsubsection{The inhibition of nuclear export is abrogated in Nup214 aa 1916-2033 al mutant in vivo and in vitro}

To analyze whether the Nup214 aa 1916-2033 a1, a2 and a3 mutants inhibit nuclear export, HeLa cells were cotransfected with plasmids coding for RFP-Nup214-cNLS aa 1916-2033 WT or a1, a2, a3 mutant and GFP-SPN1 or GFP-NC2 $\beta$ (Fig. 28A and B). Apart from Nup214 WT, the a2 mutant led to nuclear accumulation of GFP-SPN1, whereas the a1 and a3 mutant showed no effect on the localization of the cargo. Interestingly, the distribution of GFP-NC2 $\beta$ was mildly affected in the presence of Nup214 a1 mutant, as it was more equally distributed between the nucleus and the cytoplasm compared to cells expressing RFP-cNLS. Only the mutation of all FGs in Nup214 could completely restore the cytoplasmic localization of GFP-NC2 $\beta$. Thus, effects on localization of NC2 $\beta$ correlated with nuclear accumulation of CRM1 in the presence of the a1- and a3- mutant. 
(A)
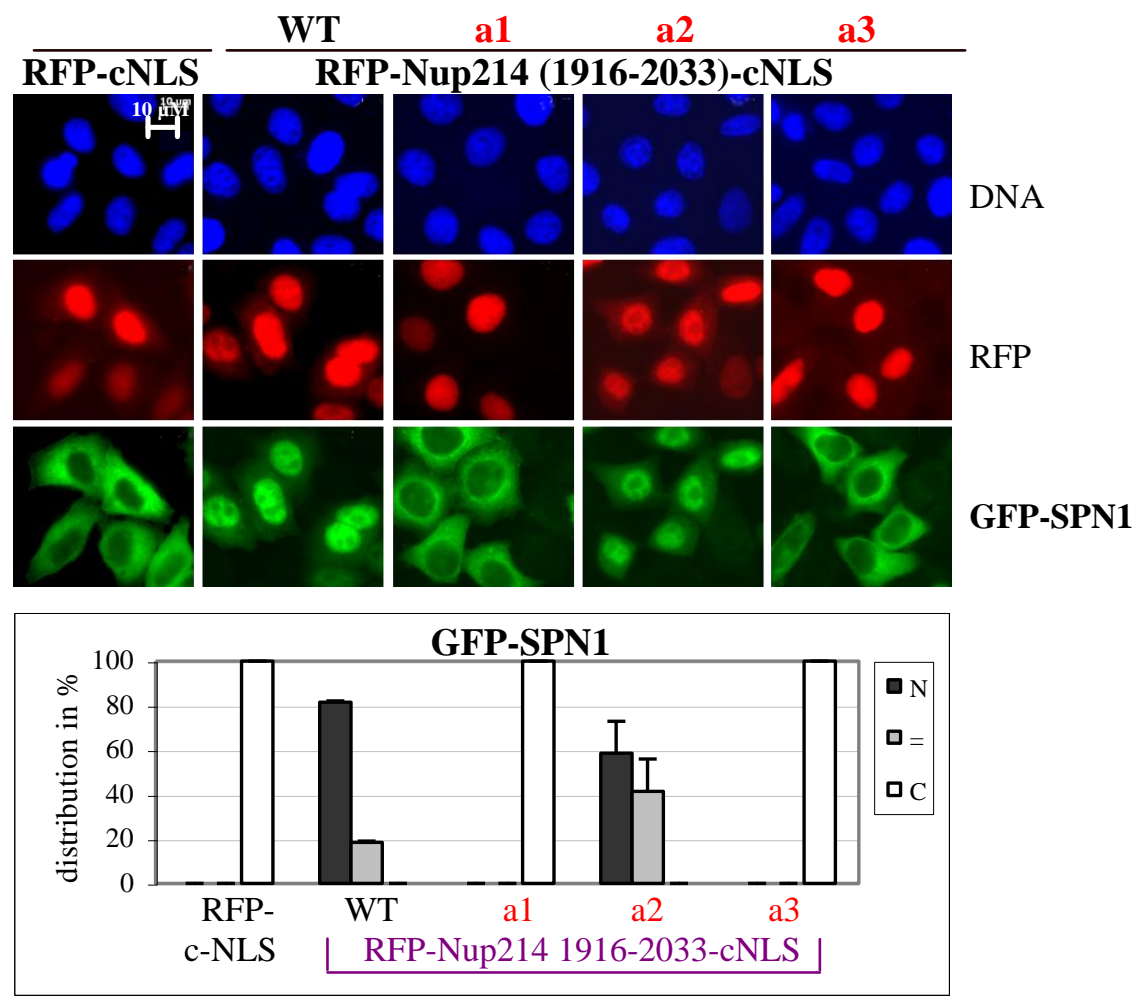

(B)
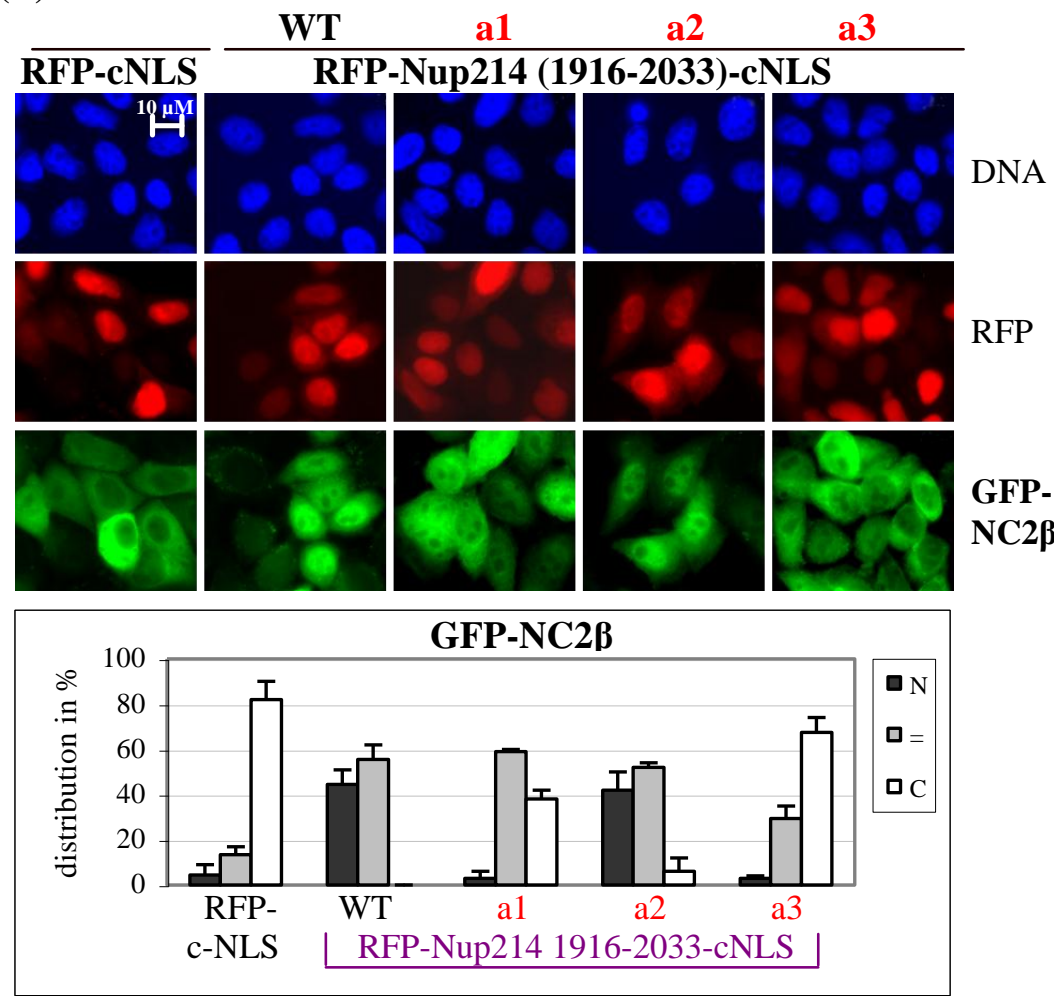

Fig. 28 Nuclear export can take place in the presence of Nup214 1916-2033 a1 mutant in vivo. HeLa cells were cotransfected with plasmids coding for RFP-Nup214 aa 1916-2033 WT or a1, a2 or a3 mutant and GFP-SPN1 (A) or GFP-NC2 $\beta$ (B), respectively. After 20 hours, the cells were fixed and analyzed by immuno-fluorescence microscopy. The distribution (in \%; $\mathrm{N}$ nuclear, = equally distributed, $\mathrm{C}$ cytoplasmic) of GFP-SPN1 and GFPNC2 $\beta$ in cells expressing RFP-Nup214-cNLS aa 1916-2033 WT or a1, a2, a3 mutant was quantified by counting at least 100 cells. Error bars indicate the variation from the mean of two independent experiments. 
To analyze differences in the effect of Nup214 aa 1916-2033 WT and a1 mutant on nuclear export, transport reactions with His-Nup214-fragments were carried out in permeabilized GFP-NFAT cells (Fig. 29). Cytosol, Ran and increasing amounts of either Nup214 aa 1916-2033 WT or a1 mutant were added. For Nup214 WT the same results could be obtained as described before (compare Fig. 22). Strikingly, in the presence of even a high concentration of the Nup214 a1 mutant, nuclear fluorescence of GFP-NFAT was low and comparable to fluorescence in cells treated with cytosol and Ran (see red frame). Thus, nuclear export of GFP-NFAT is inhibited in the presence of Nup214 aa 1916-2033 WT, but not in the presence of the a1 mutant. Taken together, the four FGs in the FG-motif are needed to inhibit nuclear export.

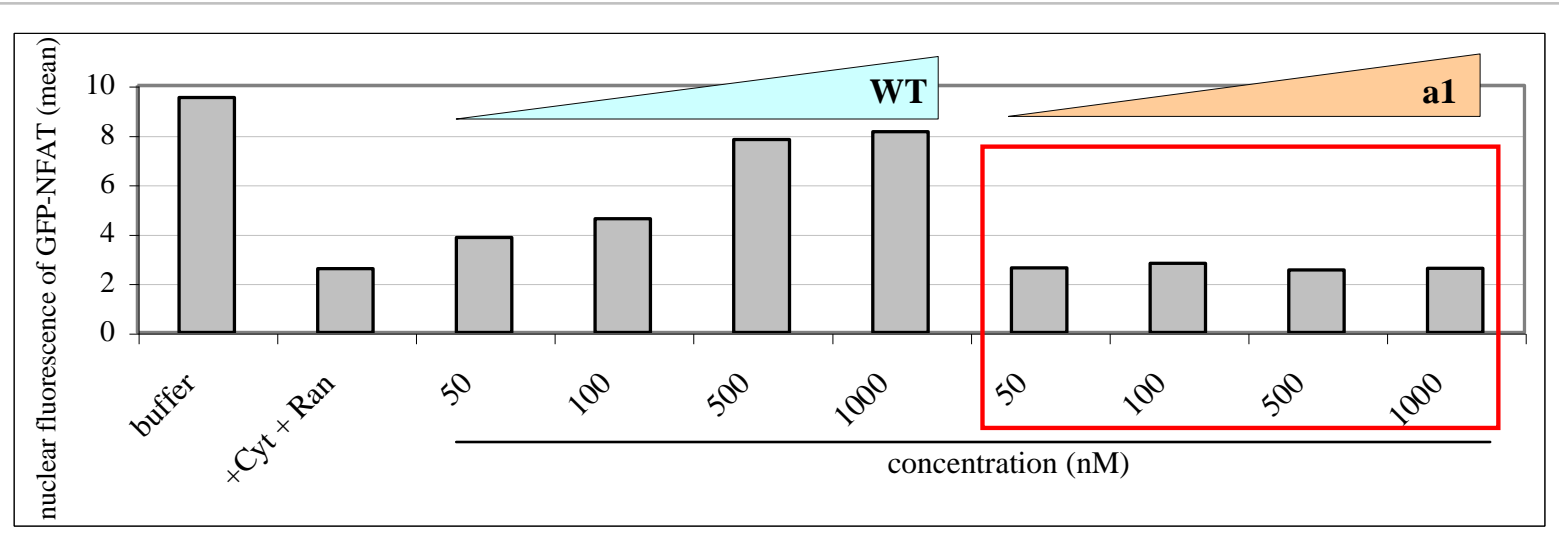

Fig. 29 Nuclear export is not inhibited in the presence of Nup214 1916-2033 a1 mutant in vitro. Stable GFP-NFAT HeLa cells were permeabilized with digitonin and subjected to export reactions in vitro. Cytosol, Ran and different concentrations of His-Nup214 aa 1916-2033 WT or a1 were added as indicated. The nuclear fluorescence of GFP-NFAT was analyzed by flow cytometry.

\subsubsection{Analysis of the Nup214 FG-motif in Nup214 aa 1859-2090}

The data presented before indicated that the FG-motif is a very strong binding signal for CRM1. Now we analyzed whether the mutation of the 4 FGs would have the same effect in the context of Nup214 aa 1859-2090, as it was observed for Nup214 aa 1916-2033. For this purpose, mutations were introduced in RFP-Nup214 (1859-2090)-cNLS and HeLa cells were cotransfected with plasmids coding for this mutant and GFP-NES, GFP-SPN1 or GFP-NC2 2 .

In cells overexpressing Nup214 a1 mutant, GFP-NES was equally distributed between the nucleus and the cytoplasm, whereas GFP-SPN1 clearly accumulated in the nucleus (Fig. 30A). GFP-NC2 $\beta$ was detected in the nucleus but also distributed equally between the nucleus and the cytoplasm. The localization of the cargo proteins in the presence of the Nup214 aa 1859-20090 a1 mutant was comparable to that in cells overexpressing Nup214 aa 1859-2090 WT (Fig. 30B). Furthermore, 
endogenous CRM1 accumulated in the nucleus in the presence of RFP-Nup214 1859-2090-cNLS a1 mutant (Fig. 30C), as it was observed for Nup214 aa 1859-2090 WT (Fig. 21, fragment 1).

Thus, in Nup214 aa 1859-2090, the FGs in the FG-motif are not sufficient to affect nuclear export. The retention of CRM1 in the nucleus in the presence of the Nup214 a1 mutant indicates that this mutant can still bind CRM1. This further supports the idea that there are other regions in the Cterminus of Nup214, which are able to interact with CRM1.

(A)

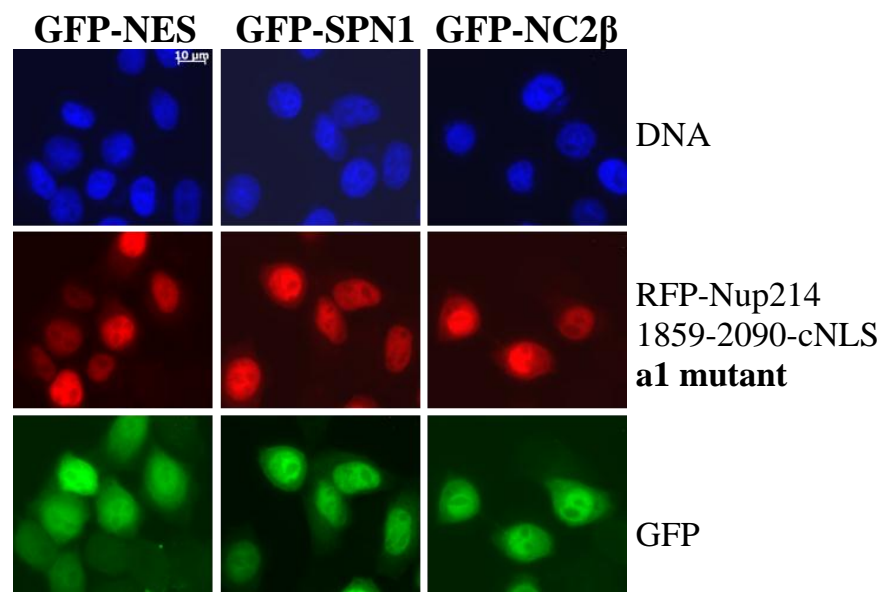

(C)
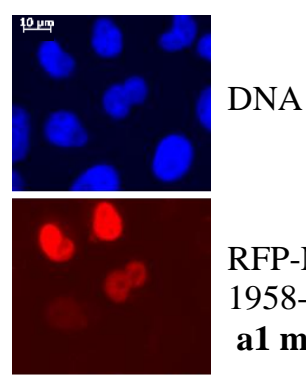

RFP-Nup214 1958-2090-cNLS a1 mutant

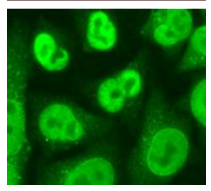

endogenous CRM1
(B)
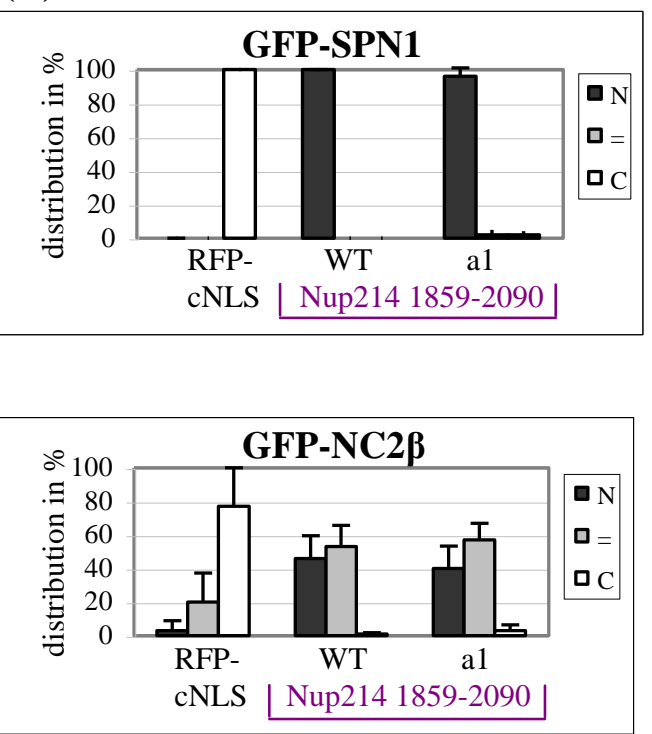

Fig. 30 Export cargos accumulate in the nucleus in the presence of Nup214 1859-2090 a1 mutant. (A) HeLa cells were cotransfected with plasmids coding for RFP-Nup214 1859-2090 a1 mutant and GFP-NES, GFP-SPN1 or GFP-NC2 $\beta$. After 20 hours the cells were fixed and analyzed by immunofluorescence microscopy. (B) The distribution (in \%; $\mathrm{N}$ nuclear, = equally distributed, C cytoplasmic) of GFP-SPN1 and GFP-NC2 $\beta$ was quantified by counting at least 100 cells. Bars indicate the variation from the mean of two independent experiments. (C) Nup214 a1 mutant over-expressing HeLa cells were fixed and stained for endogenous CRM1. 


\subsubsection{Refined search for CRM1-binding sites in Nup214}

The collected data so far indicated a CRM1-binding region in Nup214 aa 1975-2033, whereas the tested Nup214-fragment aa 1859-1974 is dispensable for CRM1 interaction. Furthermore, Nup214 aa 1975-2033 also contains the main part of the highly conserved FG-motif (aa 1970-1990). However, a Nup214 aa 1859-2090 mutant containing SGs instead of FGs in this motif behaves like Nup214 WT. The FG-motif seems to be an important platform for CRM1, but taken together, our data point to at least two CRM1-binding sites in Nup214. In this section, the very last part of the Nup214 C-terminal region (aa 1968-2090) was investigated by analyzing and comparing very short Nup214-fragments.

\subsubsection{The FG-motif in Nup214 requires aa 1991-2033 to form a stable complex with CRMI and RanGTP}

In order to identify other regions in Nup214 which bind to CRM1, GST-Nup214-fragments (WT or a1 mutant) were constructed and purified as depicted in Fig. 31A. Furthermore, RanGTP- and substratedependent binding of CRM1 to Nup214 was investigated.

Nup214 aa 1859-2090 interacts with CRM1 in a RanGTP-dependent manner (Fornerod et al., 1996, Kehlenbach et al., 1999). In the first approach, Ran-dependent binding of CRM1 to the shorter GSTNup214-fragments was analyzed. The fragments as indicated were bound to glutathione sepharose beads and CRM1, SPN1 and Ran, loaded either with GDP or GTP, were added. Gst-Nup214 aa 19681990 WT (the FG-motif) and a1 mutant were not able to bind CRM1 in the presence of RanGTP (Fig. 31B). Strikingly, GST-Nup214 aa 1968-2033 WT bound the CRM1-RanGTP-SPN1-export complex, whereas GST-Nup214 aa 1968-2033 a1 mutant did not bind to any of the components (see red frame). Notably, Nup214 aa 1991-2033 alone was not able to bind to the export complex. Taken together, Nup214 aa 1991-2033 is required for interaction of CRM1 with the FG-motif.

Binding of CRM1 to Nup214 aa 1859-2090 is enhanced in the presence of an export cargo (Kehlenbach et al., 1999). In the second approach, CRM1 and RanGTP binding to the shorter Nup214-fragments were analyzed in the presence or the absence of the cargo SPN1 (Fig. 31C). Basically, only GST-Nup214 aa 1968-2033 WT could bind CRM1 (see red frame), as it was observed in the approach described before. This binding increased in the presence of SPN1. Thus, enhanced binding of CRM1 to Nup214 aa 1859-2090 in the presence of an export cargo is also observed for the short Nup214-fragment aa 1968-2033.

Taken together, these results give evidence for the importance of the FG-motif for CRM1-interaction in a RanGTP- and export cargo- dependent manner. However, Nup214 aa 1991-2033 strongly contributes to this binding. 
(A)

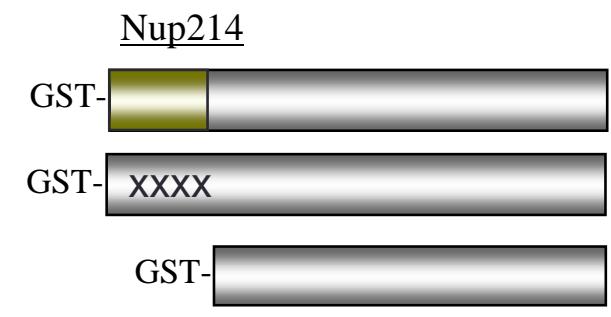

GST-

GST- XXXX

\section{8-2033 WT}

1968-2033 a1 (four FGs in the FG-motif $\rightarrow$ SGs)

$1991-2033$

1968-1990 WT

1968-1990 a1 (four FGs in the FG-motif $\rightarrow$ SGs)

(B)

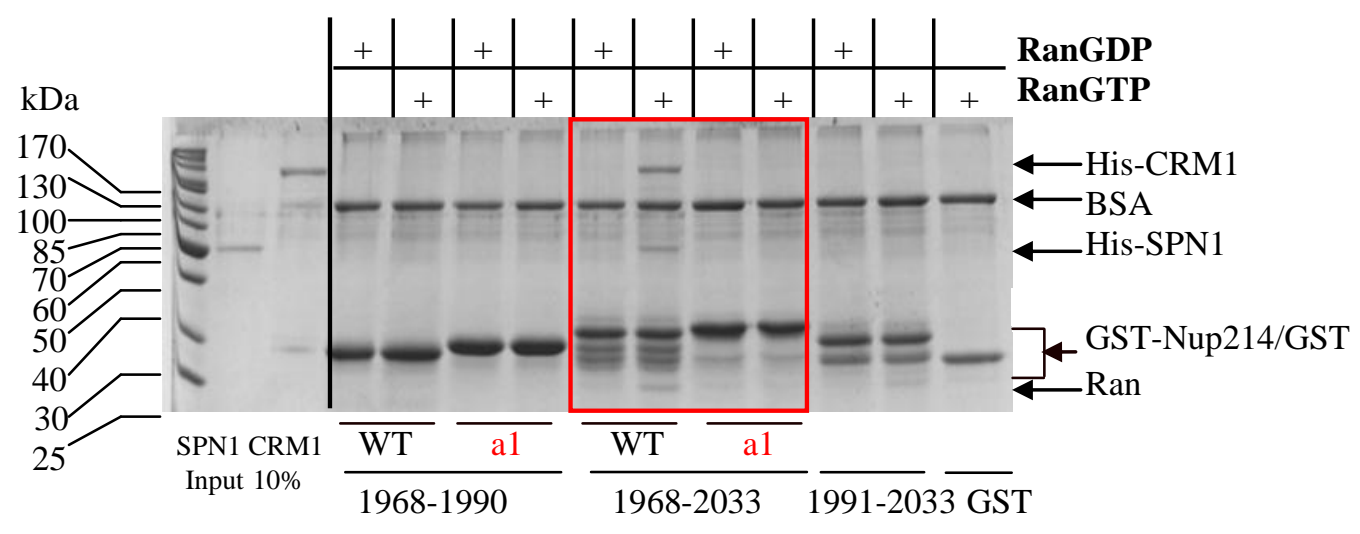

(C)

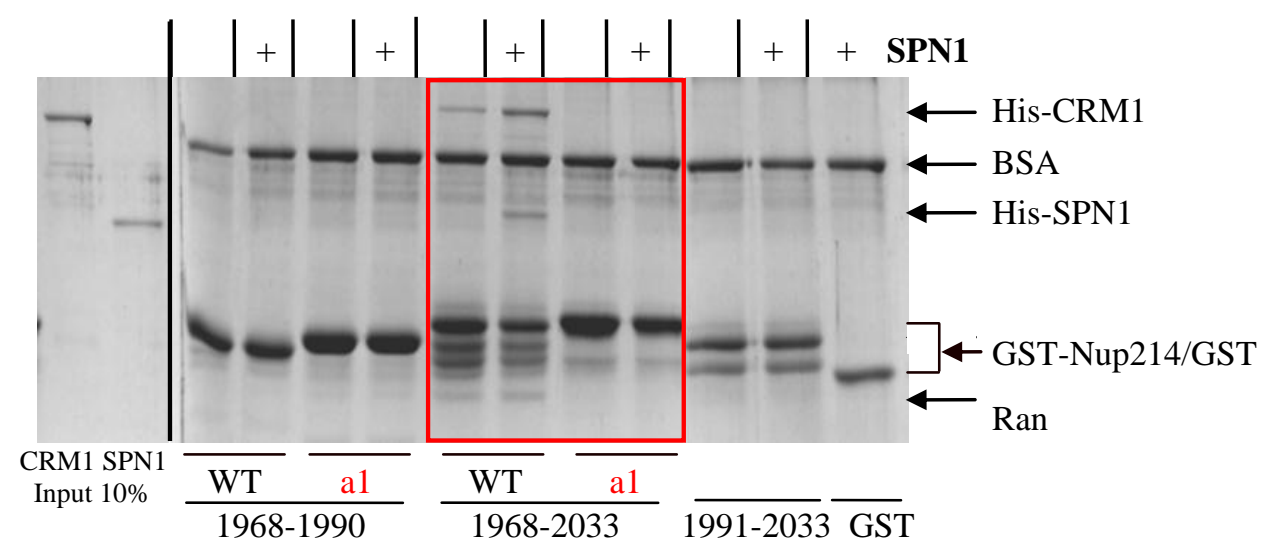

Fig. 31 The RanGTP- and cargo- dependent interaction of CRM1 with the FG-motif (aa 1968-1990) in Nup214 is stabilized by the region downstream of this motif (aa 1991-2033). GST-Nup214-fragments (5 $\mu \mathrm{g})$ as indicated in (A) were immobilized on glutathione sepharose beads. They were incubated with (B) His-CRM1 $(5 \mu \mathrm{g})$, His-SPN1 $(5 \mu \mathrm{g})$ and Ran $(10 \mu \mathrm{g})$ loaded with either GDP or GTP, or with (C) His-CRM1, RanGTP and w/o His-SPN1. BSA was used for blocking unspecific binding sites. Binding of the components was analyzed by SDS-PAGE and coomassie staining. 


\subsubsection{Elongation of Nup214 C-terminal fragments leads to increasing functionality in nuclear export}

In order to test which regions around the FG-motif (aa 1968-1990) in Nup214 affect nuclear export of proteins, RFP-cNLS Nup214-fragments were constructed as indicated in Fig. 32A. GFP-NC2 $\beta$ was chosen as a substrate because, compared to SPN1 (see appendix, Fig. S2), it reacts more sensitive to overexpression of Nup214-fragments. HeLa cells were cotransfected with plasmids coding for RFPNup214-cNLS and GFP-NC2 $\beta$, and export inhibition was analyzed by immunofluorescence microscopy. First, the cells were grouped as usual into cells with nuclear $(\mathrm{N})$ or cytoplasmic $(\mathrm{C})$ located GFP-NC2 $\beta$ or cells with GFP-NC2 $\beta$ distributed equally between the nucleus and the cytoplasm (=). For better comparison of the effects of the Nup214-fragments on GFP-NC2 $\beta$, the ratio of the nuclear distribution of GFP-NC2 $\beta$ in RFP-Nup214-cNLS expressing cells and the nuclear distribution of GFP-NC2 $\beta$ in RFP-cNLS expressing cells was determined.

In the presence of the RFP-Nup214-cNLS fragments aa 1859-2090, 1975-2090 and 1916-2033, the number of cells with nuclear located GFP-NC2 $\beta$ increased 5-8 fold compared to RFP-cNLS alone (Fig. 32B). Notably, Nup214 aa 1859-2090 and 1975-2090 showed the strongest export inhibition effect, whereas Nup214 aa 1859-1974 did not influence the localization of GFP-NC2 $\beta$. Stepwise truncation of Nup214 aa 1975-2090 (fragments aa 1991-2090 and 2034-2090; see group I in Fig. 32A, also underlined in Fig. 32B) led to a stepwise decrease of cells showing nuclear GFP-NC2 $\beta$ (from 7 to 2 fold). Notably, the lack of the FG-motif in Nup214 aa 1991-2090 already influenced localization of the cargo, and a further shortening to aa 2034-2090 led to comparable amounts of cells with nuclear located GFP-NC2 $\beta$ in the presence of RFP-cNLS alone. Interestingly, for GFP-SPN1, Nup214 aa 1991-2090 was the only fragment lacking the FG-motif and inhibiting nuclear export at the same time (see appendix, Fig. S2), and truncation of this fragment at the N-terminus (lack of aa 1991-2033) completely abolished export inhibition of GFP-SPN1.

In principle, the same effect on nuclear export of GFP-NC2 $\beta$ was observed for N-terminal truncations of Nup214 aa 1916-2033 (group II in Fig 32A and B; Nup214 aa 1968-2033 and 1991-2033). However, Nup214 aa 1916-1990, compared to RFP-cNLS alone, did not affect the localization of GFP-NC2 $\beta$ although it contains the FG-motif. On the other hand, Nup214 aa 1968-2033, also containing the FG-motif, led to a clear nuclear accumulation of GFP-NC2 $\beta$, though the effect was weaker compared to the Nup214-fragment aa 1916-2033. The FG-motif alone (aa 1968-1990) had no effect on nuclear export of the cargo. Together, the in vivo data confirmed the results obtained from the in vitro pulldown assay (compare Fig. 31).

In summary, there are regions in Nup214 contributing to the function of the FG-motif (aa 1968-1990). These regions are located mainly downstream of this motif. The data strongly suggest that the region between aa 1991 and 2033 plays the most prominent role in supporting CRM1-binding to the FGmotif. Nevertheless, also the region before (aa 1916-1967) seems to influence functionality of the FG- 
motif, as removing this region from Nup214 aa 1916-2033 (resulting in Nup214 aa 1968-2033) led to decreased nuclear accumulation of GFP-NC2 $\beta$.

(A)

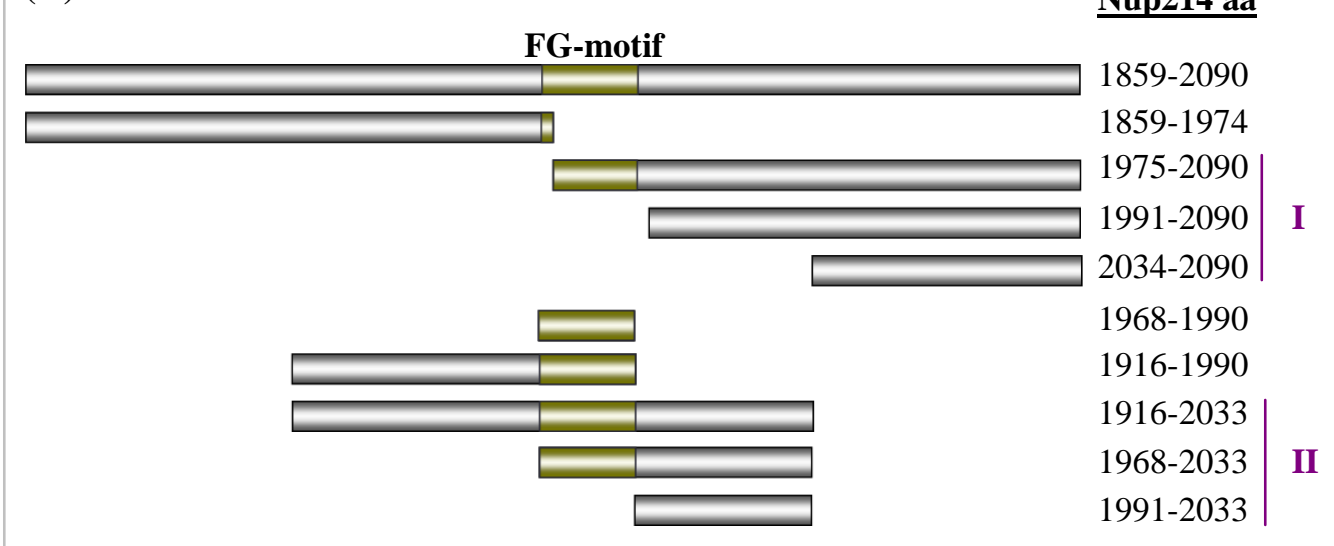

(B)

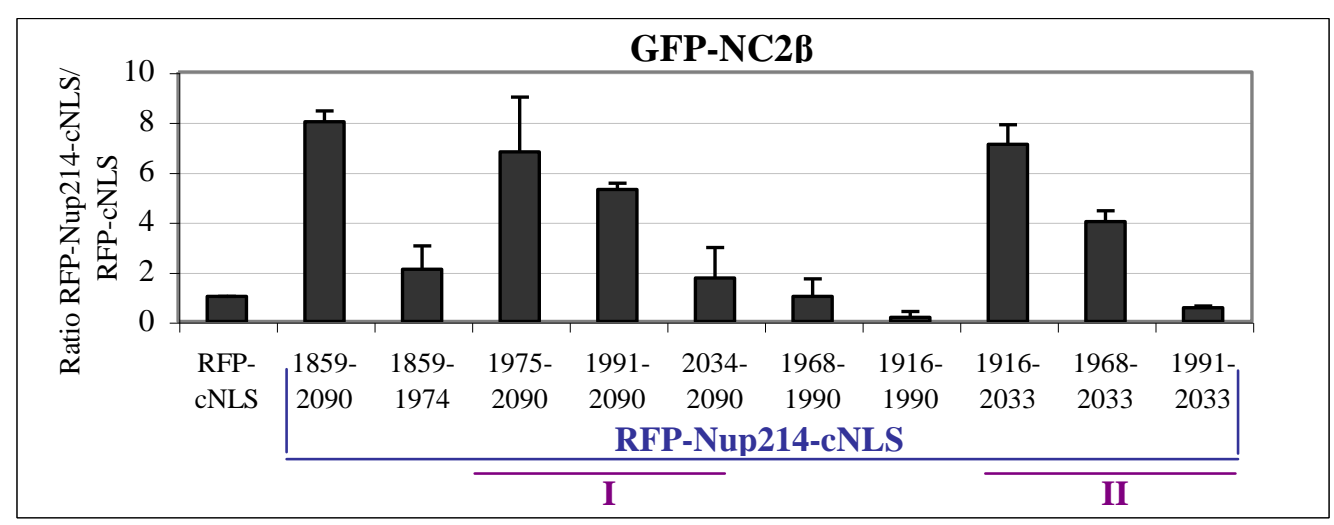

Fig. 32 Effect of short Nup214-fragments on nuclear accumulation of GFP-NC2及. (A) RFPNup214-cNLS fragments were constructed as depicted. (B) HeLa cells were cotransfected with plasmids coding for RFP-Nup214-cNLS-Fragments and GFP-NC2 $\beta$. After 20 hours the cells were fixed and distribution of GFP-NC2 $\beta$ was quantified by counting at least 60 cells. The ratio of the nuclear distribution of GFP-NC2 $\beta$ in the presence of RFP-Nup214-cNLS and in the presence of RFP-cNLS was determined. The bars indicate the variation from the mean of two independent experiments.

Altogether, we identified a major CRM1-binding site in Nup214 which is also responsible for nuclear export inhibition of cargo proteins. This FG-motif in Nup214 additionally requires aa 1991-2033 for a stable interaction with CRM1. Nup214 aa 1991-2090, which does not contain the FG-motif, inhibited nuclear export of GFP-NC2 $\beta$ even more efficiently compared to Nup214 aa 1968-2033, which does contain the FG-motif. When removing aa 1991-2033 from Nup214 aa 1991-2090, nuclear export inhibition was completely abrogated. Thus, Nup214 aa 1991-2033 not only contributes to the function of the FG-motif in aa 1968-1990, but also to the function of an unidentified CRM1-binding region in Nup214 aa 2034-2090. 


\section{Discussion}

\subsection{Analysis of CRM1-fragments and -chimeras}

\section{Inhibition of CRM1 by LMB as an alternative approach to CRM1 RNA interference}

RNA interference is a common method to investigate the effect of proteins in eukaryotic cells and also in whole organisms (Fire et al., 1998). This system takes advantage of the protective mechanism of cells against viruses or transposons (Waterhouse et al., 2001). Eukaryotic cells or organisms are exposed to small RNAs with sequences complementary to the mRNA transcript of the protein to be depleted. However, off-target effects are common, because already a match of 11-15 nucleotides can lead to unspecific gene suppression (Jackson et al., 2003). Furthermore, the interferon-system can be activated and protein biosynthesis can be inhibited via activation of the RNA-dependent protein kinase PKR and phosphorylation of the transcription factor eIF2 (eukaryotic initiation factor 2; Levin et al., 1978). Additionally, RNase $\mathrm{L}$ is induced, resulting in degradation of mRNA and apoptosis (Pandey et al., 2004). Together, the effects, which are observed upon depletion of proteins of interest, always have to be analyzed with respect to specificity. In the case of CRM1, other problems could potentially arise upon RNA interference. CRM1 is the main export receptor and transports hundreds of cargos out of the nucleus (la Cour et al., 2003). Hence, depletion of CRM1 by RNA interference would cause mislocalization of many proteins, finally causing a lot of side effects. The usage of the LMB-method allows cells to grow under normal conditions. Unlike the siRNA treatment, which takes several days, LMB is added to cells for only a few hours, minimizing potential side effects. Furthermore, LMB is a selective inhibitor, as it only modifies CRM1 (Kudo et al., 1999) by binding covalently to the hydrophobic cleft, that is also used by the NES-sequences of CRM1-dependent cargos.

In analogy to RNA interference experiments, where siRNA resistant mutants can be expressed in cells upon depletion of endogenous proteins, CRM1, containing a point mutation in the NES-binding cleft, can be overexpressed together with the cargo protein of interest. LMB covalently modifies C528 in CRM1, and the mutation of this amino acid residue leads to LMB resistant, but still functional CRM1. The big advantage of this approach is switching off endogenous CRM1, but at the same time giving the cells the chance to use exogenous CRM1 C528S. Indeed, in LMB-treated HeLa cells, that overexpress CRM1 C528S, the localization of the tested NES-cargos could be rescued. Together, the LMB approach is an efficient tool to analyze CRM1-dependent localization of proteins. We set out to use this mutation in CRM1-fragments and chimeras. This enabled us to investigate the function of distinct regions in CRM1, without having background effects produced by endogenous CRM1. 


\section{Nucleoporin binding and export capability of CRM1-fragments and -chimeras}

Based on our previous knowledge of domains in CRM1, their assigned functions and the available crystal structure (Petosa et al., 2004), the N-terminus of CRM1 was responsible for Ran binding, and the central part was binding to NES-cargos (Nazari and Dargemont, 1999). The function of the C-terminal half of CRM1 was not known. To analyze this region, we constructed HA-CRM1fragments lacking C-terminal HEAT repeats. As CRM1 interacts with several nucleoporins like Nup153, p62 and also with Nup214 in a RanGTP-dependent manner (Fornerod et al., 1996; Kehlenbach et al., 1999), we assumed that nucleoporin-binding could occur in the C-terminal region and performed coimmunoprecipitation experiments with the CRM1-fragments. Although endogenous CRM1 could be coimmunoprecipitated with HA-Nup214 (Fornerod et al. 1996), we had problems to coimmunoprecipitate endogenous Nup214 with HA-CRM1-fragments. In general, the expression level of HA-CRM1 constructs is low in HeLa cells. Endogenous CRM1, present in higher amounts in total cell lysates, could saturate the binding sites in endogenous Nup214 molecules and therefore compete with HA-CRM1. We therefore resorted to a microscopic analysis and showed that none of the tested CRM1 fragments associated with the nuclear envelope. This correlated with their inability to export cargos out of the nucleus. During our work on CRM1-fragments, the crystal structure of CRM1 in a trimeric export complex was solved (Dong et al., 2009; Monecke et al., 2009), showing CRM1 as a ring-like protein. RanGTP is located in the cavity of this ring, connected to several HRs of CRM1. In CRM1-fragments, this ring structure might be disturbed, thus affecting RanGTP-binding. Due to this, export complex formation as well as the interaction with nucleoporins could be affected. Besides problems in nucleoporin-interaction that might occur with CRM1-fragments, it is also unclear whether the release of the export cargo via RanBP1 into the cytoplasm is possible. The acidic $\alpha$-helix at the very C-terminus of CRM1, which modulates the affinity of CRM1 to NES-cargos (Dong et al., 2009; Fox et al., 2011), is missing in all CRM1-fragments. Both, NES-cargo and RanBP1, affect RanGTP binding to CRM1.

The inability of CRM1-fragments to associate with the nuclear envelope emphasizes the specific role of RanGTP in CRM1-nucleoporin interaction. The function of this interaction is to facilitate translocation of transport complexes through the nuclear pore, and the translocation process itself has been discussed in several models (Macara, 2001; Ribbeck and Görlich, 2001; Rout et al., 2003; Yamada et al., 2010). To date, the structure of unbound CRM1 is not known but it is presumed that due to RanGTP-binding, CRM1 undergoes conformational changes, additionally promoting export cargo binding (Monecke et al., 2009). It is also not known, whether binding of nucleoporins influences the conformation of CRM1.

To overcome problems of RanGTP-/cargo- binding to CRM1, translocation through the nuclear pore and release of the export complex in the cytoplasm, we decided to continue our studies with $H$. sapiens/C. elegans CRM1-chimeras. There is no crystal structure available for C. elegans CRM1, but we assume that it is present as a ring-like protein with similar binding properties for an NES-cargo, 
RanGTP and RanBP1, too. One striking difference between C. elegans CRM1 and human CRM1 is the inability to interact with human nucleoporins (S. Roloff, 2008, Diploma thesis). Because of this, C. elegans CRM1 should not be able to fully mediate export through the nuclear pore. A GFP-NES was exported by $C$. elegans CRM1 to a certain extent. By contrast, $C$. elegans CRM1 was neither able to export human GFP-SPN1 nor GFP-NC2 $\beta$. The residual export activity, however, showed that C. elegans CRM1 can interact with human RanGTP as well as with the GFP-NES reporter. The crystal structure of the CRM1-RanGTP-SPN1-complex (Monecke et al., 2009) indicates more than one binding site for SPN1 on CRM1. Thus, $C$. elegans CRM1 is presumably not able to fulfil all required binding conditions for SPN1. Besides the lack of appropriate nucleoporin binding sites in C. elegans CRM1, its minimal export capability for a GFP-NES in human cells might be due to the fact that this NES interacts only with the hydrophobic NES-cleft and with nothing else. The reason for nuclear accumulation of GFP-NC2 $\beta$ remains unclear, as the binding mechanism to CRM1 is not known. Due to the restricted export capability and inability to interact with human nucleoporins, C. elegans CRM1 was a suitable candidate for analysis of CRM1-chimeras.

Among our chimeras, we found one that acted similar to human CRM1 in mammalian cells. Chimera HsCe I (human HR 1-11 and C. elegans HR 12-21) was located in the nucleus and at the nuclear envelope and could mediate export of at least a subset of cargo proteins. GFP-NES and GFP-SPN1 could be efficiently exported, so we can conclude that $\mathrm{Hs} C \mathrm{I}$ contains all binding regions for SPN1. Furthermore, the chimera-export complex must contain nucleoporin binding regions and must also be able to disassemble in the cytoplasm. Based on our approaches, we cannot say whether this chimera and human CRM1 have similar affinities for interacting export factors, but we see that $\mathrm{HsCe} \mathrm{I}$ is functional in mammalian cells. NC2 $\beta$, by contrast, accumulated in the nucleus in the presence of HsCe I under conditions where endogenous CRM1 is inhibited by LMB, indicating a binding mechanism to CRM1, different from that of GFP-SPN1 or GFP-NES. Obviously, these binding properties are not present in the $C$. elegans CRM1 part. Hence, important NC2 $\beta$ binding regions might be found in the C-terminus of human CRM1. Strikingly, the chimera CeHs I (with C. elegans CRM1 in the N-terminal half and $H$. sapiens CRM1 at the C-terminal half) was clearly restricted in its export capability as none of the tested cargos could be exported. When we assume that important binding regions for NC2 $\beta$ are located in the C-terminus of CRM1, the export complex should be assembled with this chimera without difficulties. This would also be true for SPN1, which interacts with human CRM1 HR 14A, 15A and 16A (Monecke et al., 2009). As CeHs I did not show a rim-staining around the nucleus and neither could mediate nuclear export of the tested cargos, we assume that association with the nuclear pore is related to the ability of CRM1 to mediate export of cargos into the cytoplasm. Assuming a proper ring closure and the ability to interact with human RanGTP and an NES-cargo, missing binding regions for nucleoporins could explain the export restriction for this chimera. Hence, binding sites for human nucleoporins like Nup214, the terminal docking site for export complexes (Fornerod et al., 1996; Kehlenbach et al., 1999), are likely present in the N-terminal portion of CRM1. 
Our assumption is further supported by identified nucleoporin-binding sites in importin $\beta$, also located in the N-terminus (Kutay et al., 1997).

Consistent with their inability to associate to the nuclear envelope, none of the chimeras type II could mediate export in mammalian cells. CeHs II (C. elegans HR 1-8 and human HR 9-21) and HsCe II were equally distributed in the nucleus and the cytoplasm. In both chimeras putative nucleoporin binding sites are missing or affected. Comparing CRM1 chimeras $\mathrm{HsCe}$ I and $\mathrm{HsCe}$ II, the exchange of $H$. sapiens HRs 9-11 into C. elegans HRs was sufficient to abrogate association with the nuclear envelope. From this, we conclude that HRs 9-11 of human CRM1 are important for interaction with nucleoporins. We could not detect CRM1-chimera - nucleoporin interaction in coimmunoprecipitation experiments. For $\mathrm{HsCe} \mathrm{I}$, we hypothesize a RanGTP-dependent binding to nucleoporins. The composition of this chimera would indicate nucleoporin interaction at the N-terminal part of human CRM1. However, we cannot exclude a decreased affinity of this chimera for RanGTP, which would explain the lack of nucleoporin binding in coimmunoprecipitation. Additionally, the exchange of human CRM1 regions into C. elegans CRM1 parts could lead to structural or conformational changes. Considering these aspects, a potential binding to nucleoporins could not be seen because available endogenous nucleoporins would mostly bind to endogenous human CRM1. To further investigate HR 9-11 in CRM1, binding studies with recombinant CRM1-chimeras have to be performed.

Nup214 interacts with full-length human CRM1 in a RanGTP-dependent manner (Fornerod et al., 1996) and this binding is enhanced in the presence of an NES (Kehlenbach et al., 1999), indicating a cooperative binding mechanism of all four components. When we assume an interaction of Nup214 with HRs 9-11 in CRM1, this cooperativity can be explained by the close proximity of Nup214binding to the Ran- and cargo- relevant regions in CRM1. In Fig. 33, a model is depicted, in which Nup214-binding involves several HEAT repeat regions nearby the RanGTP- and cargo- binding sites of CRM1. Such a Nup214- interaction would specify the CRM1-RanGTP-cargo export complex as such in the nuclear pore. The function of this interaction will be discussed in the following section. 


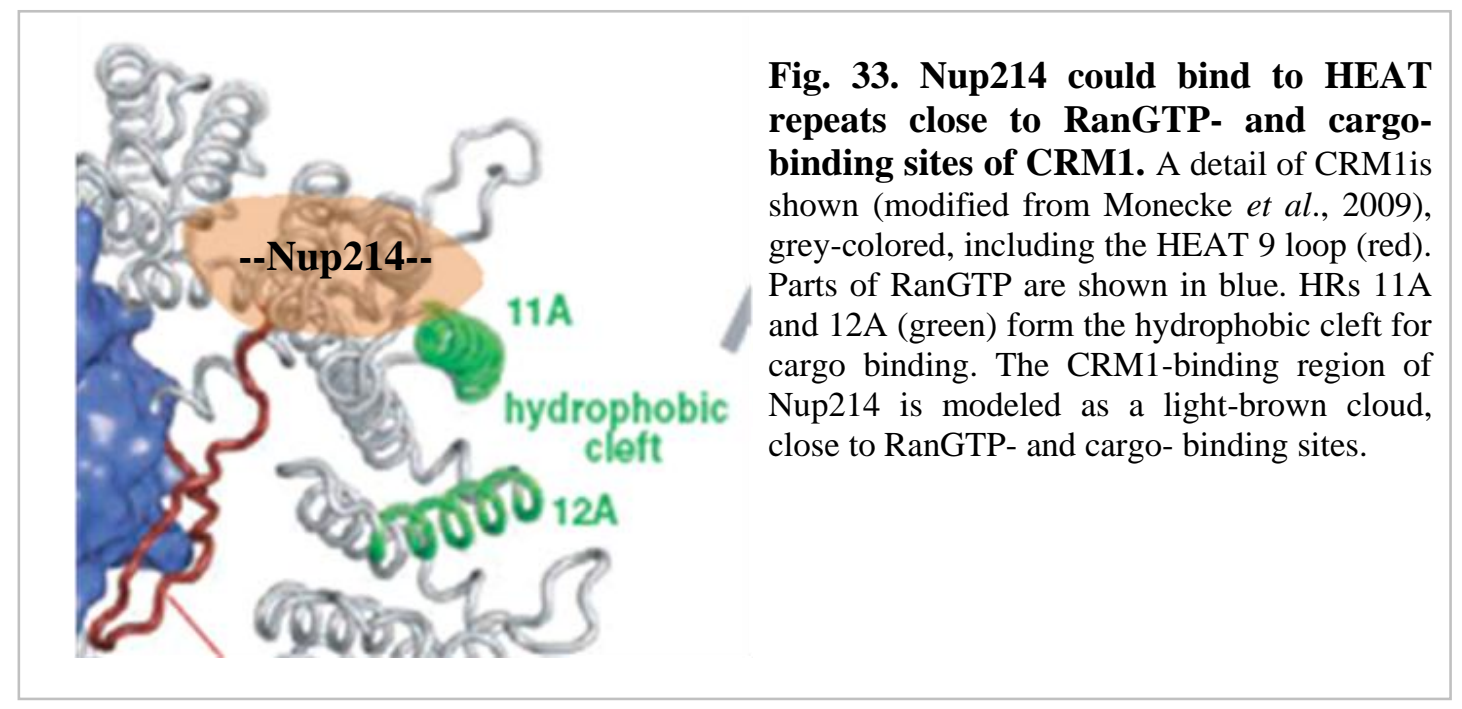

\subsection{Analysis of the CRM1-binding region in Nup214}

\section{The interaction of CRM1 with Nup214 is based on FGs}

In coimmunoprecipitation experiments, the FG-rich C-terminus of Nup214 (aa 1859-2090) was identified as the CRM1-binding site, and smaller fragments from this region failed to coprecipitate CRM1 (Fornerod et al., 1996). Data from Boer et al. (1998) presented C-terminal Nup214 (aa 18642090) and a deletion mutant (aa 1140-1340, 1864-1912, 1984-2090) which colocalized with endogenous CRM1. Here, we could narrow down the regions in Nup214 that are responsible for CRM1-accumulation in the nucleus of cells. Moreover, we could show that this accumulation correlated with the ability of Nup214-fragments to bind to CRM1 in pulldown-assays. Notably, in cells overexpressing CRM1-binding Nup214-fragments, nuclear export of NES-containing cargos was affected. but not nuclear import. Hence, impaired nuclear export seems to be the result of direct binding of endogenous CRM1 to overexpressed Nup214-fragments. It is believed that CRM1 interacts with the FGs in nucleoporins, like other transport receptors. Upon transfection of C-terminal Nup214 mutant lacking all FGs, CRM1 did not accumulate in the nucleus. This demonstrates that the FGs in Nup214 are responsible for CRM1-binding. However, CRM1 does not bind to any FG-rich region, as the Nup214-fragment aa 1859-1974, containing eight FGs, was not able to interact with CRM1.

In Nup214-fragments that bound to CRM1, we found a prominent FG-motif (aa 1970-1990) consisting of four FGs in an identical distance to each other: (FG $\times x \mathbf{P} \times)_{\mathbf{4}}$. Mutations of the FGs in this motif in short Nup214-fragments led to severe CRM1-binding defects. Furthermore, nuclear accumulation of 
CRM1 and export cargos was abrogated. In the context of the longer Nup214 C-terminal region (aa 1859-2090), mutation of the FG-motif caused nuclear accumulation of CRM1 similar to Nup214 WT, suggesting that this mutant also binds CRM1. In combination with the results we got from other short Nup214-fragments, we conclude that not only the FG-motif binds to CRM1, but that there are two other binding sites in Nup214, stabilizing this interaction. Nup214 aa 1991-2033 contributes to CRM1-binding to the FG-motif but also to an unidentified region between aa 2034 and 2090 (Fig 34). The smallest fragment lacking the FG- motif but inhibiting nuclear export was Nup214 aa 1991-2090.

Importin $\beta$ has been shown to bind via several regions to nucleoporins (Bayliss et al., 2000; Kutay et al., 1997b). Also in the nuclear import factor p97 more than one nucleoporin-binding site was found (Chi and Adam, 1997). Together with our results obtained from Nup214 fragments, also in CRM1 more than one binding region for Nup214 must be present.

\begin{tabular}{|l|l|l|l|}
\hline & $\begin{array}{l}\text { Fig. 34 Nup214 binds with at } \\
\text { least three regions to CRM1. } \\
\text { The trimeric export complex (shown } \\
\text { in blue colours) interacts with several } \\
\text { regions of Nup214. The FG-motif (aa }\end{array}$ \\
$\begin{array}{l}\text { 1970-1990; yellow) binds to CRM1. } \\
\text { Nup214 aa 1991-2033 (orange) and } \\
\text { another unidentified region (XXX) } \\
\text { stabilize this interaction. }\end{array}$
\end{tabular}

The role of the FGs in the terminal steps of nuclear export

The cytoplasmically located Nup214 serves as a terminal docking site in the late steps of nuclear export. RanBP1 releases CRM1 from Nup214 (Kehlenbach et al., 1999), indicating a role of Nup214 preparing the export complex for the following releasing steps by RanGAP. RanBP1 is a soluble protein, and RanGAP can be soluble, but is also associated to Nup358 (Mahajan et al. 1997; Matunis et al. 1996; Weis 2007). Due to the strong binding to Nup214, export complexes containing CRM1 could be concentrated at the nuclear pore, thus allowing efficient disassembly by RanBP1 and RanGAP.

For importin $\beta$ it is known that its binding pockets for FGs have different binding properties and sensitivities to RanGTP (Otsuka et al., 2008). Consequently, the impact of RanGTP on distinct FGbinding regions in CRM1 could also modulate the affinity of CRM1 for Nup214. Moreover, RanGTPdependent binding of FGs in Nup214 to CRM1 could induce conformational changes in the export complex, which might be crucial for the following releasing steps. The identification of the FG-motif with its contributing C-terminal FGs of Nup214 strongly suggests a defined binding pattern for 
CRM1. This might be important for a correct orientation of the export complex at the cytoplasmic side of the NPC, thus allowing the presentation of distinct CRM1 regions to releasing factors like RanGAP. The FG-motif also contains prolines which have an identical distance to each other. Prolines have the potential to cause nicks in $\alpha$-helices. FG-regions in Nups are intrinsically unstructured (Allen et al., 2002; Denning et al., 2003; Patel et al., 2007), and we do not know which kind of structure the FGmotif would have when bound to CRM1. Thus, it is unclear what function these prolines could have. Nup214-fragments where the prolines are exchanged for alanines in the FG-motif can still bind to CRM1, and export cargos also accumulate in the presence of this mutant.

Controversially to all observations concerning the function of Nup214 in nuclear export, namely to be involved in releasing the cargo from the CRM1 export complex, only a few cargos have been shown to be CRM1- and Nup214- dependent. They have been identified via overexpression in Nup214 depleted cells (Bernad et al., 2006; Hutten and Kehlenbach, 2006), and an accumulation of these proteins in the nucleus had been observed. However, cargos that are nuclear per se, due to a stronger import signal compared to the export signal, cannot be investigated with this method. Consequently, alternative ways have to be found to analyze the impact of Nup214 on CRM1-dependent cargos. In this work, nuclear accumulation of CRM1, due to short overexpressed Nup214-fragments, led to problems in cell growth. This had also been described for the complete FG-containing C-terminal part of Nup214 aa 1859-2090 (Boer et al., 1998). Moreover, depletion of Nup214 leads to reduced cell growth, too (Hutten and Kehlenbach, 2006). Overexpression of the Nup214-Nup88 complex reduces CRM1 from the nuclear pore, resulting in impaired nuclear export (Xylourgidis et al., 2006). All these findings imply a general role of Nup214-CRM1 interaction in nuclear export. The identification of the Nup214binding site in CRM1 and of export cargos that are dependent on both, Nup214 and CRM1, would help to elucidate the role of Nup214 in CRM1-mediated nuclear export. 


\subsection{Outlook}

To test whether the binding of Nup214 occurs in HRs 9-11 in CRM1, we have to optimize protein purification of CRM1-chimeras and do binding assays with them. Furthermore, the construction of triple chimeras (e.g. H.sapiens HR 1-8/C. elegans HR 9-11/H. sapiens HR 12-21) can give us further insight into the function of certain HRs in vivo.

The FG-motif in Nup214 seems to be the most important binding site for CRM1, and at least two distinct regions in Nup214 contribute to this interaction. For identification of the binding sites, Nup214-fragments with mutations in selected FG-areas will be constructed and analyzed in vivo and in vitro. Cross-linking of suitable Nup214-fragments with CRM1 should be performed to identify the CRM1-binding region for Nup214. The ultimate goal is to obtain co-crystals of Nup214-fragments and the CRM1-export complex and to determine their structure by X-ray crystallography. Furthermore, Nup214- and CRM1- dependent export cargos will have to be systematically investigated, e.g. by screening for cytoplasmic proteins that accumulate in the nucleus upon depletion of Nup214 (similar to the approach that has been used for Nup358 (Wälde et al., 2011)).

The elucidation of the role for CRM1-Nup214 interaction would also help to get more insight into the function of other nucleoporins in nuclear transport. The uncoverage of similarities or differences in transport receptor-nucleoporin interaction might lead to a better understanding, how transport complexes translocate through the nuclear pore. 


\section{References}

Adachi, Y. and M. Yanagida. 1989. Higher order chromosome structure is affected by cold-sensitive mutations in Schizosaccharomyces pombe gen CRM1+ which encodes a $115-\mathrm{kD}$ protein preferentially localized in the nucleus and its periphery. J.Cell.Biol. 108:1195-1207.

Alber, F., S. Dokudovskaya, L.M. Veenhoff, W. Zhang, J. Kipper, D. Devos, A. Suprapto,O. Karni-Schmidt, R. Williams, B.T. Chait, M.P. Rout and A. Sali. 2007. Determining the architectures of macromolecular assemblies. Nature. 450:683-94.

Allen, N. P., S. S. Patel and M. Rexach. 2002. Deciphering networks of protein interactions at the nuclear pore complex. Mol. Cell. Proteomics. 1:930-946.

Andrade, M.A., and P. Bork. 1995. HEAT repeats in the Huntington's disease protein. Nat Genet. 11:115-6.

Arts, G.J., M. Fornerod and I.W. Mattaj. 1998. Identification of a nuclear export receptor for tRNA. Curr Biol. 8:305-314.

Askjaer, P., A. Bachi, M. Wilm, F. R. Bischoff, D. L. Weeks, V. Ogniewski, M. Ohno, C. Niehrs, J. Kjems, I. W. Mattaj and M. Fornerod. 1999. RanGTP-regulated interactions of CRM1 with nucleoporins and a shuttling DEAD-box helicase. Mol. Cell. Biol. 19:6276-6285.

Ausubel, F.M., R. Brent, R.E. Kingston, D.D. Moore, J.G. Seidman, J.A. Smith and K. Struhl. 1994. Current protocols in molecular biology. New York: Greene. Publishing Associates and Wiley-Interscience.

Bayliss, R., K, Ribbeck, D, Akin, H.M. Kent, C.M. Feldherr, D. Görlich and M. Stewart. 1999. Interaction between NTF2 and xFxFG-containing nucleoporins is required to mediate nuclear import of RanGDP. J. Mol. Biol. 293:579-593.

Bayliss R, T. Littlewood and M. Stewart. 2000. Structural basis for the interaction between FxFG nucleoporin repeats and importin-beta in nuclear trafficking. Cell 102:99-108.

Bayliss R, T, Littlewood, L.A. Strawn, S.R. Wente and M. Stewart. 2002. GLFG and FxFG nucleoporins bind to overlapping sites on importin-beta. J. Biol. Chem. 277:50597-606.

Bastos, R., L. Ribas de Pouplana, M. Enarson, K. Bodoor and B. Burke. 1997. Nup84, a novel nucleoporin that is associated with CAN/Nup214 on the cytoplasmic face of the nuclear pore complex. J Cell Biol. 137:989-1000.

Beals, C.R., N.A. Clipstone, S.N. Ho and G.R. Crabtree. 1997a. Nuclear localization of NF-ATc by a calcineurindependent, cyclosporin-sensitive intramolecular interaction. Genes Dev 11:824-834.

Beals, C.R., C.M. Sheridan, C.W. Turck, P. Gardner and G.R. Crabtree. 1997b. Nuclear export of NF-ATc enhanced by glycogen synthase kinase-3. Science 275:1930-1934.

Beck, M., F. Forster, M. Ecke, J.M. Plitzko, F. Melchior, G. Gerisch, W. Baumeister and O. Medalia. 2004. Nuclear Pore Complex Structure and Dynamics Revealed by Cryoelectron Tomography. Science. 306:1387-1390.

Becker, J., F. Melchior, V. Gerke, F.R. Bischoff, H. Ponstingl and A. Wittinghofer. 1995. RNA1 encodes a GTPaseactivating protein specific for Gsp1p, the Ran/TC4 homologue of Saccharomyces cerevisiae J. Biol. Chem. 270:11860-11865.

Ben-Efraim, I. and L. Gerace. 2001. Gradient of increasing affinity of importin beta for nucleoporins along the pathway of nuclear import. J Cell Biol. 152:411-417.

Bernad, R., H. van der Velde, M. Fornerod and H. Pickersgill. 2004. Nup358/RanBP2 attaches to the nuclear pore complex via association with Nup88 and Nup214/CAN and plays a supporting role in CRM1-mediated nuclear protein export. Mol. Cell. Biol. 24:2373-2384.

Bernad, R., D. Engelsma, H. Sanderson, H. Pickersgill and M. Fornerod. 2006. The Nup214-Nup88 nucleoporin subcomplex is required for CRM1-mediated 60 S preribosomal nuclear export. J Biol Chem. 28:19378-86.

Bischoff, F. R. and H. Ponstingl. 1991. Catalysis of guanine nucleotide exchange on Ran by the mitotic regulator RCC1. Nature. 354: 80-82. 
Bischoff, F. R., H. Krebber, E. Smirnova, W. Dong and H. Ponstingl. 1995. Co-activation of RanGTPase and inhibition of GTP dissociation by Ran-GTP binding protein RanBP1. EMBO J. 14:705-715.

Boer, J., J. Bonten-Surtel and G. Grosveld. 1998. Overexpression of the Nucleoporin CAN/NUP214 Induces Growth Arrest, Nucleocytoplasmic Transport Defects, and Apoptosis. Mol. Cell. Biol. 18:1236-1247.

Bogerd, H.P., R.A. Fridell, R.E. Benson, J. Hua and B.R. Cullen. 1996. Protein sequence requirements for function of the human T-cell leukemia virus type 1 Rex nuclear export signal delineated by a novel in vivo randomizationselection assay. Mol. Cell. Biol. 16:4207-4214.

Cang, Y. and G. Prelich. 2002. Direct stimulation of transcription by negative cofactor 2 (NC2) through TATA binding protein (TBP). PNAS. 99 (20):12727-12732.

Carmody, S.R. and S.R.Wente. 2009. mRNA nuclear export at a glance. J Cell Sci 122:1933-1937.

Chadrin, A., B. Hess, M. San Roman, X. Gatti, B. Lombard, D. Loew, Y. Barral, B. Palancade and V. Doye. 2010. Pom33, a novel transmembrane nucleoporin required for proper nuclear pore complex distribution. J. Cell Biol. 189:795-811.

Charneau, P., G. Mirambeau, P. Roux, S. Paulous, H. Buc and F. Clavel. 1994. HIV-1 reversetranscription. A termination step at the center of the genome. J Mol Biol.241:651-62.

Clarke, P. and Z. Chuanmao. 2008. Spatial and temporal coordination of mitosis by Ran GTPase. Nat. Rev. Mol. Cell Bio. 9:464-477

Chi, N.C. and S.A. Adam. 1997. Functional domains in nuclear import factor p97 for binding the nuclear localization sequence receptor and the nuclear pore. Mol Biol Cell. 8(6):945-956.

Chook, Y.M., and G. Blobel. 1999. Structure of the nuclear transport complex karyopherin-beta2-RanGppNHp. Nature. 399: 230-237.

Conti, E. and J. Kuriyan. 2000. Crystallographic analysis of the specific yet versatile recognition of distinct nuclear localization signals by karyopherin $\alpha$. Structure. 8:329-38.

Conti, E. and E. Izaurralde. 2001. Nucleocytoplasmic transport enters the atomic age. Curr Opin Cell Biol 13: 310-9.

Cook, A., F. Bono, M. Jinek and E. Conti. (2007). Structural biology of nucleocytoplasmic transport. Annu Rev Biochem 76: 647-671

la Cour, T., R. Gupta, K. Rapacki, K. Skriver, F.M. Poulsen and S. Brunak. 2003. NESbase version 1.0, a database of Nuclear Export Signals. Nucleic Acids Res. 31(1):393-396.

Cronshaw, J.M., A.N. Krutchinsky, W. Zhang, B.T. Chait and M.J. Matunis. 2002. Proteomic analysis of the mammalian nuclear pore complex. J Cell Biol. 158:915-27.

Denning, D.P., S.S. Patel, V. Uversky, A.L. Fink and M. Rexach. 2003. Disorder in the nuclear pore complex: the FGrepeat regions of nucleoporins are natively unfolded. Proc Natl Acad Sci U S A. 100:2450-5.

van Deursen, J., J. Boer, L. Kasper and G. Grosveld. 1996. G2 arrest and impaired nucleocytoplasmic transport in mouse embryos lacking the proto-oncogene CAN/Nup214. EMBO J. 15(20):5574-5583.

Devos, D., S. Dokudovskaya, R. Williams, F. Alber and N. Eswar. 2006. Simple fold composition and modular architecture of the nuclear pore complex. Proc. Natl. Acad. Sci. USA 103:2172-77.

Dingwall, C., S.V. Sharnick and R.A. Laskey. 1982. A polypeptide domain that specifies migration of nucleoplasmin into the nucleus. Cell. 30:449-458.

Dong, X., A. Biswas and Y.M. Chook. 2009. Structural basis for assembly and disassembly of the CRM1 nuclear export complex. Nat. Struct. Mol. Biol. 16:558-560.

Englmeier, L., M. Fornerod, F.R. Bischoff, C. Petosa and I.W. Mattaj. 2001. RanBP3 influences interactions between CRM1 and its nuclear protein export substrates. EMBO Rep 2: 926-932.

Fahrenkrog, B., J. Koser and U. Aebi. 2004. The nuclear pore complex: a jack of all trades? Trends Biochem Sci. 29:175-82. 
Finlay, D.R., D.D. Newmeyer, T.M. Price and D.J. Forbes. 1987. Inhibition of in vitro nuclear transport by a lectin that binds to nuclear pores. J. Cell Biol. 104, 189-200.

Fischer, U., J. Huber, W.C. Boelens, I.W. Mattaj and R. Lührmann. 1995. The HIV-1 Rev activation domain is a nuclear export signal that accesses an export pathway used by specific cellular RNAs. Cell. 82:475-83.

Fire A, S. Xu, M.K. Montgomery, S.A. Kostas, S.E. Driver and C.C. Mello. 1998. Potent and specific genetic interference by double-stranded RNA in Caenorhabditis elegans. Nature 391:806-11.

Fontes, M.R., T. Teh and B. Kobe. 2000. Structural basis of recognition of monopartite and bipartite nuclear localization sequences by mammalian importin $\alpha$. J Mol Biol. 297:1183-94.

Fornerod, M., J. Boer, S. van Baal, H. Morreau and G. Grosveld. 1996. Interaction of cellular proteins with the leukemia specific fusion proteins DEK-CAN and SET-CAN and their normal counterpart, the nucleoporin CAN. Oncogene 13:1801-1809.

Fornerod, M., J. van Deursen, S. van Baal, A. Reynolds, D. Davis, K. G. Murti, J. Fransen and G. Grosveld. 1997a. The human homologue of yeast CRM1 is in a dynamic subcomplex with CAN/Nup214 and a novel nuclear pore component Nup88. EMBO J. 16:807-816.

Fornerod, M., M. Ohno, M. Yoshida and I. W. Mattaj. 1997b. CRM1 is an export receptor for leucine-rich nuclear export signals. Cell 90:1051-1060.

Fox, A.M., D. Ciziene, S.H. McLaughlin and M. Stewart. 2011. Electrostatic Interactions Involving the Extreme C Terminus of Nuclear Export Factor CRM1 Modulate Its Affinity for Cargo. Biol Chem. 286(33): 29325-29335.

Frey, S., R. P. Richter and D. Görlich. 2006. FG-rich repeats of nuclear pore proteins form a three-dimensional meshwork with hydrogel-like properties. Science 314: 815-817.

Frey, S. and D. Görlich. 2007. A Saturated FG-Repeat Hydrogel Can Reproduce the Permeability Properties of Nuclear Pore Complexes.Cell 130:512-523.

Fribourg, S., I.C. Braun, E. Izaurralde and E. Conti. 2001. Structural basis for the recognition of a nucleoporin FG repeat by the NTF2-like domain of the TAP/p15 mRNA nuclear export factor. Mol. Cell 8:645-56.

Fukuda, M., L. Gotoh, M. Adachi, Y. Gotoh and E. Nishida. 1997: A novel regulatory mechanism in the mitogenactivated protein (MAP) kinase cascade. Role of nuclear export signal of MAP kinase kinase. J. Biol. Chem. 272 (5): 32642-32648.

Gadal, O., D. Strauss, J. Kessl, B. Trumpower, D. Tollervey and E. Hurt. 2001. Nuclear export of 60s ribosomal subunits depends on Xpo1p and requires a nuclear export sequence-containing factor, Nmd3p, that associates with the large subunit protein Rpl10p. Mol. Cell. Biol. 21:3405-3415.

Geisberg, J. V., F.C. Holstege, R.A. Young and K. Struhl. 2001. Yeast NC2 associates with the RNA polymerase II preinitiation complex and selectively affects transcription in vivo. Mol Cell Biol, 21(8):2736-42.

Gerace, L., Y. Ottaviano and C. Kondor-Koch. 1982. Identification of a major polypeptide of the nuclear pore complex. J. Cell Biol. 95:826-837.

Görlich, D., S. Kostka, R. Kraft, C. Dingwall, R.A. Laskey, E. Hartmann and S. Prehn. 1995a. Two different subunits of importin cooperate to recognize nuclear localization signals and bind them to the nuclear envelope. Curr. Biol. 5:383-392.

Görlich, D., F. Vogel, A.D. Mills, E. Hartmann and R.A. Laskey. 1995b. Distinct functions for the two importin subunits in nuclear protein import. Nature. 377:246-8.

Grauxl, C., J. Cools, C. Melotte1, H. Quentmeier, A. Ferrando, R. Levine, J.R. Vermeesch1, M. Stul1, B. Dutta, N. Boeckx, A. Bosly, P. Heimann, A. Uyttebroeck, N. Mentens, R. Somers, R.A.F. MacLeod, H.G. Drexler, A.T. Look, D.G. Gilliland, L. Michaux, P. Vandenberghe, I. Wlodarska, P. Marynen and A. Hagemeijer. 2004. Fusion of NUP214 to ABL1 on amplified episomes in T-cell acute lymphoblastic leukaemia. Nature. 36:1084-1089. 
Guan, T., R. H. Kehlenbach, E. C. Schirmer, A. Kehlenbach, F. Fan, B. E. Clurman, N. Arnheim, L. Gerace. 2000. Nup50, a Nucleoplasmically Oriented Nucleoporin with a Role in Nuclear Protein Export. Mol Cell Biol. 20:56195630 .

Güttler, T., T. Madl, P. Neumann, D. Deichsel, L. Corsini, T. Monecke, R. Ficner, M. Sattler and D. Görlich. 2010. NES consensus redefined by structures of PKI-type and Rev-type nuclear export signals bound to CRM1. Nature. 17:1367-1377.

Hallberg, E., R.W. Wozniak and G. Blobel. 1993. An integral membrane protein of the pore membrane domain of the nuclear envelope contains a nucleoporin-like region. J. Cell Biol. 122:513-521.

Hamada, M., A. Haeger, K.B. Jeganathan, J.H. van Ree, L. Malureanu, S. Wälde, J. Joseph, R.H. Kehlenbach and J.M. van Deursen. 2011. Ran-dependent docking of importin-beta to RanBP2/Nup358 filaments is essential for protein import and cell viability. J. Cell Biol. 194:597-612.

Hilliard, M., C. Frohnert, C. Spillner, A. Nath, T. Lampe, S. Marcone, D. Fitzgerald and R.H. Kehlenbach. 2010. The anti-inflammatory prostaglandin 15-Deoxy-D ${ }^{12,14} \mathrm{PGJ}_{2}$ inhibits CRM1-dependent nuclear protein export. J. Biol. Chem. 285:22202-22210.

Hodel, M.R., A.H. Corbett and A.E. Hodel. 2001. Dissection of a nuclear localization signal. J Biol Chem. 276:1317-25.

Hoelz, A., E.W. Debler and G. Blobel. 2011. The Structure of the Nuclear Pore Complex. Annu. Rev. Biochem.80:613-643.

Hofmann, W., B. Reichart, A. Ewald, E. Müller, I. Schmitt, R.H. Stauber, F. Lottspeich, B.M. Jokusch, U. Scheer, J. Hauber and M.C. Dabauvalle. 2001. Cofactor Requirements for Nuclear Export of Rev Response Element (Rre)-And Constitutive Transport Element (Cte)-Containing Retroviral Rnas. J Cell Biol. 152(5):895-910.

Holt, G.D., C.M. Snow, A. Senior, R.S. Haltiwanger, L. Gerace and G.W. Hart. 1987. Nuclear pore complex glycoproteins contain cytoplasmically disposed O-linked N-acetylglucosamine.J Cell Biol. 104:1157-64.

Huber, J., U. Cronshagen, M. Kadokura, C. Marshallsay, T. Wada, M. Sekine and R. Lührmann. 1998. Snurportin 1, an $\mathrm{m} 3 \mathrm{G}-\mathrm{cap}-$ specific nuclear import receptor with a novel domain structure. EMBO (Eur. Mol. Biol. Organ.) $J$. 17:4114-4126.

Hutten, S. and R. H. Kehlenbach. 2006. Nup214 Is Required for CRM1-Dependent Nuclear Protein Export In Vivo. Mol. Cell. Biol. 28:6772-6785.

Hutten, S., A. Flotho, F. Melchior and R. H. Kehlenbach. 2008. The Nup358-RanGAP Complex Is Required for Efficient Importin $\alpha / \beta$-dependent Nuclear Import. Mol Biol Cell. 19: 2300-2310.

Isgro, T.A. and K. Schulten. 2007a. Cse1p-binding dynamics reveal a binding pattern for FG-repeat nucleoporins on transport receptors.Structure 15: 977-991.

Isgro, T.A. and K. Schulten. 2007b. Association of nuclear pore FG-repeat domains to NTF2 import and export complexes. J. Mol.Biol. 366: 330-345.

Izaurralde, E., U. Kutay, C. von Kobbe, I.W. Mattaj and D. Görlich. 1997. The asymmetric distribution of the constituents of the Ran system is essential for transport into and out of the nucleus. EMBO J. 16:6535-6547.

Jackson, A.L., S.R. Bartz, J. Schelter, S.V. Kobayashi, J. Burchard, M. Mao, B. Li, G. Cavet and P.S. Linsley. 2003. Expression profiling reveals off-target gene regulation by RNAi. Nat Biotechnol. 21:635-7.

Johnson, A.W., E. Lund and J. Dahlberg. 2002. Nuclear export of ribosomal subunits. Trends Biochem. Sci.27:580585.

Kahle, J., E. Piaia. S. Neimanis, M. Meisterernst and D. Doenecke. 2009. Regulation of Nuclear Import and Export of Negative Cofactor 2. J Biol Chem. 284:9382-9393

Kalderon, D., W.D. Richardson, A.F. Markham and A.E. Smith. 1984. Sequence requirements for nuclear location of simian virus 40 large $\mathrm{T}$ antigen. Nature. 311:33-8.

De Keersmaecker, J.L. R. Rocnik, B.H. Bernad, D. Lee, O. Leeman. H. Gielen, C. Verachtert, S. Folens, K. Munck, P. Marynen, M. Fornerod and J. Cools. 2008. Kinase Activation and Transformation by NUP214-ABL1 Is Dependent on the Context of the Nuclear Pore. Mol. Cell. 1(31): 134-142. 
Kehlenbach, R.H., A. Dickmanns and L. Gerace. 1998. Nucleocytoplasmic Shuttling Factors Including Ran and CRM1 Mediate Nuclear Export of NFAT In Vitro. J. Cell. Biol. 141:863-874.

Kehlenbach, R.H., A. Dickmanns, A. Kehlenbach, T. Guan and L. Gerace. 1999. A role for RanBP1 in the release of CRM1 from the nuclear pore complex in a terminal step of nuclear export. J. Cell Biol. 145:645-657.

Kehlenbach, R.H., R. Assheuer, A. Kehlenbach, J. Becker and L. Gerace. 2001. Stimulation of Nuclear Export and Inhibition of Nuclear Import by a Ran Mutant Deficient in Binding to Ran-binding Protein 1 .J. Biol. Chem. 276:14524-14531.

Keminer O. and R. Peters. 1999. Permeability of single nuclear pores. Biophys. J. 77:217-228.

Kim, V.N. 2004. MicroRNA precursors in motion: exportin-5 mediates their nuclear export. Trends Cell Biol. 14:156-159.

Kosugi, S., M. Hasebe, M. Tomita and H. Yanagawa. 2008. Nuclear export signal consensus sequences defined using a localization-based yeast selection system. Traffic 9:2053-2062.

Kraemer, D., R.W. Wozniak, G. Blobel and A. Radu. 1994. The human CAN protein, a putative oncogene product associated with myeloid leukemogenesis, is a nuclear pore complex protein that faces the cytoplasm. Proc Natl Acad Sci U S A. 91:1519-23.

Kudo, N., N. Matsumori, H. Taoka, D. Fujiwara, E. S. Schreiner, B. Wolff, M. Yoshida and S. Horinouchi. 1999. Leptomycin B inactivates CRM1/exportin 1 by covalent modification at a cysteine residue in the central conserved region. Cell Biol. 96:9112-9117.

Kurisaki, A., K. Kurisaki, M. Kowanetz, H. Sugino, Y. Yoneda, C.H. Heldin and A. Moustakas. 2006. The Mechanism of Nuclear Export of Smad3 Involves Exportin 4 and Ran. Mol. Cell Biol. 26(4):1318-1332.

Kutay, U., F.R. Bischoff, S. Kostka, R. Kraft and D. Görlich. 1997a. Export of importin alpha from the nucleus is mediated by a specific nuclear transport factor. Cell 90: 1061-1071.

Kutay, U., E. Izaurralde, F.R. Bischoff, I.W. Mattaj and D. Görlich. 1997b. Dominant-negative mutants of importin- $\beta$ block multiple pathways of import and export through the nuclear pore complex. EMBO. 16:1153-1163.

Kutay, U., G. Lipowsky, E. Izaurralde, F.R. Bischoff, P. Schwarzmaier, E. Hartmann and D. Görlich. 1998. Identification of a tRNA-specific nuclear export receptor. Mol. Cell 1 (3): 359-369.

Laemmli, U.K. 1970. Cleavage of structural proteins during assembly of the head of bacteriophage T4. Nature 227:680685.

Lee, J.B., A.E. Cansizoglu, K.E. Süel, T.H. Louis, Z. Zhang and Y.M. Chook. 2006. Rules for Nuclear Localization Sequence Recognition by Karyopherin $\beta 2$. Cell. 126: 543-558.

Lei, E.P. and P.A. Silver. 2002. Protein and RNA export from the nucleus. Dev Cell. 2:261-72.

Levin, D. and I.M. London. 1978. Regulation of protein synthesis: activation by double-stranded RNA of a protein kinase that phosphorylates eukaryotic initiation factor 2. Proc Natl Acad Sci U S A. 75:1121-5.

von Lindern, M., M. Fornerod, S. van Baal, M. Jaegle, T. de Wit, A. Buijs and G. Grosveld. 1992. The translocation (6;9), associated with a specific subtype of acute myeloid leukemia, results in the fusion of two genes, dek and can, and the expression of a chimeric, leukemia-specific dek-can mRNA.Mol. Cell. Biol. 12:1687-1697.

von Lindern, M., S. van Baal, J. Wiegant, A. Raap, A. Hagemeijer and G. Grosveld. 1992. Can, a putative oncogene associated with myeloid leukemogenesis, may be activated by fusion of its $3^{\prime}$ half to different genes: characterization of the set gene. Mol Cell Biol. 12:3346-55.

Lindsay, M. E., J. M. Holaska, K. Welch, B. M. Paschal and I. G. Macara. 2001.Ran-Binding Protein 3 Is a Cofactor for CRM1-mediated Nuclear Protein Export. J Cell Biol. 153: 1391-1402.

Lipowsky, G., F. Bischoff, P. Schwarzmaier, R. Kraft, S. Kostka, E. Hartmann, U. Kutay and D. Görlich. 2000. Exportin 4: a mediator of a novel nuclear export pathway in higher eukaryotes. EMBO J. 19: 4362-4371.

Lutzmann M, R. Kunze, A. Buerer, U. Aebi and E. Hurt. 2002. Modular self-assembly of a Y-shaped multiprotein complex from seven nucleoporins. EMBO J. 21:387-97. 
Lutzmann, M., R, Kunze, K. Stangl, P. Stelter, K.F. Tóth, B. Böttcher and E. Hurt. 2005. Reconstitution of Nup157 and Nup145N into the Nup84 complex. J Biol Chem 280: 18442-18451.

Macara, I.G. 2001. Transport into and out of the nucleus. Microbiol Mol Biol Rev. 65:570-94.

Mahajan, R., C. Delphin, T. Guan, L. Gerace and F. Melchior. 1997. A Small Ubiquitin-Related Polypeptide Involved in Targeting RanGAP1 to Nuclear Pore Complex Protein RanBP2. Cell. 88:97-107.

Mansfeld, J., S. Güttinger, L.A. Hawryluk-Gara, N. Panté M. Mall, V. Galy, U. Haselmann, P. Mühlhäusser, R.W. Wozniak, I.W. Mattaj, U. Kutay and W. Antonin. 2006. The conserved transmembrane nucleoporin NDC1 is required for nuclear pore complex assembly in vertebratecells. Mol. Cell 22: 93-103

Marg, A. Y. Shan, T. Meyer, T. Meissner, M. Brandenburg and U. Vinkemeier. 2004. Nucleocytoplasmic distribution by nucleoporins Nup153 and Nup214 and CRM1-dependent nuclear export controll the subcellular distribution of latent Stat1. J. Cell. Biol. 165(6):823-833.

Matunis, M.J., E. Coutavas and G. Blobel. 1996. A novel ubiquitin-like modification modulates the partitioning of the Ran-GTPase-activating protein RanGAP1 between the cytosol and the nuclear pore complex. J Cell Biol. 135:1457-70.

Melchior, F., D.J. Sweet and L. Gerace. 1995. Analysis of Ran/TC4 function in nuclear protein import. Methods Enzymol. 257:279-291.

Mingot, J.M., S. Kostka, R. Kraft, E. Hartmann and D. Görlich. 2001. Importin 13: a novel mediator of nuclear import and export. Embo J. 20:3685-94.

Mingot, J.M., M.T. Bohnsack, U. Jäkle and D. Görlich. 2004. Exportin 7 defines a novel general nuclear export pathway. EMBO J. 23(16): 3227-3236.

von Moeller, H., C. Basquin and E.Conti. 2009. The mRNA export protein DBP5 binds RNA and the cytoplasmic nucleoporin NUP214 in a mutually exclusive manner. Nat. Struct. Biol. 16:247-254.

Monecke, T., T. Güttler, P. Neumann, A. Dickmanns, D. Görlich and R. Ficner. 2009. Crystal Structure of the Nuclear Export Receptor CRM1 in Complex with Snurportin1 and RanGTP. Science. 324: 1087-1091.

Moore, M.S. and G. Blobel. 1994. Purification of a Ran-interacting protein that is required for protein import into the nucleus. Proc. Natl Acad. Sci. USA, 91:10212-10216.

Morinaga, Y., T. Franceschini, S. Inouye and M. Inouye. 1984. Improvement Of Oligonucleotide-Directed Site-Specific Mutagenesis Using Double-Stranded Plasmid DNA. Nat. Biotechnol. 2: 636 - 639.

Mueller, L., V.C, Cordes, F.R. Bischoff and H. Ponstingl. 1998. Human RanBP3, a group of nuclear RanGTP binding proteins. FEBS Lett 427: 330-336.

Mullis, K.B. 1990. Target amplification for DNA analysis by the polymerase chain reaction. Ann Biol Clin (Paris). 48:57982.

Nakielny, S., M.C. Siomi, H. Siomi, W.M. Michael, V. Pollard, and G. Dreyfuss. 1996. Transportin: nuclear transport receptor of a novel nuclear protein import pathway. Exp Cell Res. 229:261-6.

Nakielny, S., S. Shaikh, B. Burke and G. Dreyfuss. 1999. Nup153 is an M9-containing mobile nucleoporin with a novel Ran-binding domain. EMBO J. 18:1982-1995.

Napetschnig, J., G. Blobel and A. Hoelz. 2006. Crystal structure of the N-terminal domain of the human protooncogene Nup214/CAN. PNAS. (6) 104: 1783-1788.

Napetschnig, J., S.A. Kassube, E.W. Debler, R.W. Wong, G. Blobel and A. Hoelz. 2009. Structural and functional analysis of the interaction between the nucleoporinNup214 and the DEAD-box helicase Ddx19. PNAS. 106:3089-3094.

Nazari, B.O. and C. Dargemont. 1999. Domains of Crm1 Involved in the Formation of Crm1, RanGTP, and Leucine-Rich Nuclear Export Sequences Trimeric Complex. Exp Cell Res. 252:236-241. 
Nemergut, M.E., M.E. Lindsay, A.M. Brownawell and I.G. Macara. 2002. Ran-binding Protein 3 Links CRM1 to the Ran Guanine Nucleotide Exchange Factor J. Biol. Chem., 277:17385-17388.

Ohno, M., A. Segref, A. Bachi, M. Wilm and I.W, Mattaj. 2000. PHAX, a mediator of U snRNA nuclear export whose activity is regulated by phosphorylation. Cell. 101:187-198.

Oka, M., M. Asally, Y, Yasuda, Y. Ogawa, T. Tachibana and Y. Yoneda. 2010. The Mobile FG Nucleoporin Is a Cofactor for Crm1-dependent Protein Export. Mol. Biol. Cell. 21:1885-1896.

Otsuka, S., S. Iwasaka, Y.Yoneda, K. Takeyasu and S.H. Yoshimura. 2008. Individual binding pockets of importin-_for FG-nucleoporins have different binding properties and different sensitivities to RanGTP. PNAS. 105(42): 1610116106.

Paine, P.L., L.C. Moore and S.B. Horowitz. 1975. Nuclear envelope permeability. Nature.254:109-114.

Pandey, M., G.D. Bajaj and P.C. Rath. 2004. Induction of the interferon-inducible RNA-degrading enzyme, RNase L, by stress-inducing agents in the human cervical carcinoma cells. RNA Biol. 1:21-7.

Paraskeva, E., E. Izaurralde, F. R. Bischoff, J. Huber, U. Kutay, E. Hartmann, R. Lührmann and D. Görlich. 1999. CRM1-mediated Recycling of Snurportin 1 to the Cytoplasm. J. Cell Biol. 145:255-264.

Patel, S. S., B. J. Belmont and M. F. Rexach. 2007. Natively unfolded nucleoporins gate protein diffusion across the nuclear pore complex. Cell. 129:83-96.

Peters, R. 2005. Translocation through the nuclear pore complex: selectivity and speed by reduction-of-dimensionality. Traffic 6: $421-427$.

Petosa, C., G. Schoehn, P. Askjaer, U. Bauer, M. Moulin, U. Steuerwald, M. Soler-Lo' pez, F. Baudin, I. W. Mattaj and C.W. Müller. 2004. Architecture of CRM1/Exportin1 Suggests How Cooperativity Is Achieved during Formation of a Nuclear Export Complex. Mol. Cell.16:761-775.

Reichelt, R., A. Holzenburg, E.L. Buhle Jr, M. Jarnik, A. Engel and Aebi, U. 1990. Correlation between structure and mass distribution of the nuclear pore complex and of distinct pore complex components. J. Cell Biol.110, 883894.

Rexach, M. and G. Blobel. 1995. Protein import into nuclei: association and dissociation reactions involving transport substrate, transport factors, and nucleoporins. Cell. 83:683-92.

Ribbeck, K., G. Lipowsky, H.M. Kent, M. Stewart and D. Görlich. 1998. NTF2 mediates nuclear import of Ran. Embo J. 17:6587-98.

Ribbeck, K. and D. Görlich. 2001. Kinetic analysis of translocation through nuclear pore complexes. Embo J. 20:1320-30.

Robbins, J., S.M. Dilworth, R.A. Laskey and C. Dingwall. 1988. Two interdependent basic domains in nucleoplasmic nuclear targeting sequence: identification of a class of bipartide nuclear targeting sequence. Cell. 64:615-623.

Rodriguez, M.S., C. Dargemont and F. Stutz. 2004. Nuclear export of RNA. Biol. Cell. 96: 639-655.

Roloff, S. 2008. Die Rolle von Nup214 und seiner FG-repeats im CRM1-vermittelten Kernexport. Diploma thesis.

Roth, P., N, Xylourgidis, N. Sabri, A. Uv, M. Fornerod and C. Samakovlis. 2003. The Drosophila nucleoporin DNup88 localizes DNup214 and CRM1 on the nuclear envelope and attenuates NES-mediated nuclear export. J. Cell Biol. 163:701-706.

Rout, M.P., J.D. Aitchison, A. Suprapto, K. Hjertaas, Y. Zhao and B.T. Chait. 2000. The yeast nuclear pore complex: composition, architecture, and transport mechanism. J. Cell Biol. 148:635-651.

Rout, M.P., J.D. Aitchison, M.O. Magnasco and B.T. Chait. 2003. Virtual gating and nuclear transport: the hole picture. Trends Cell Biol. 13:622-8.

Sabri, N., P. Roth, N. Xylourgidis, F. Sadeghifar, J. Adler and C. Samakovlis. 2007. Distinct functions of the Drosophila Nup153 and Nup214 FG domains in nuclear protein transport. J. Cell Biol. 178: 557 - 565.

Saitoh, H., C.A. Cooke, W.H. Burgess, W.C. Earnshaw and M. Dasso. 1996. Direct and indirect association of the small GTPase ran with nuclear pore proteins and soluble transport factors: studies in Xenopus laevis egg extracts. Mol. Biol. Cell. 7 (9): 1319-34. 
Santos-Rosa, H., H. Moreno, G. Simos, A. Segref, B. Fahrenkrog, N. Panté and E. Hurt. 1998. Nuclear mRNA export requires complex formation between Mex67p and Mtr2p at the nuclear pores. Mol. Cell. Biol. 18:6826-6838.

Schwartz, T. U. 2005. Modularity within the architecture of the nuclear pore complex. Curr. Opin. Struct. Biol.15:221-226

Seewald, M.J., C. Korner, A. Wittinghofer and I.R. Vetter. 2002. RanGAP mediates GTP hydrolysis without an arginine finger. Nature 415:662-666.

Segref, A., K. Sharma, V. Doye, A. Hellwig, J. Huber, R. Lührmann and E. Hurt. 1997. Mex67p, a novel factor for nuclear mRNA export, binds to both poly(A)+ RNA and nuclear pores. EMBO J 16:3256-3271.

Siddiqui, N. and K.L.B. Lorden. 2012. mRNA expott and cancer. WIREs RNA. 3:13-25.

Singh, B.B., H.H. Patel, R. Roepman, D. Schick and P.A. Ferreira. 1999. The zinc finger cluster domain of RanBP2 is a specific docking site for the nuclear exprot factor exportin-1. J. Biol. Chem. 274:37370-37378.

Siniossoglou, S., C. Wimmer, M. Rieger, V. Doye, H. Tekotte, C. Weise, S. Emig, A. Segref and E.C Hurt. 1996. A novel complex of nucleoporins, which includes Sec13p and a Sec13p homolog, is essential for normal nuclear pores. Cell. 84:265-75.

Siniossoglou S, M. Lutzmann, H. Santos-Rosa, K. Leonard, S. Mueller, U. Aebi and E. Hurt. 2000. Structure and assembly of the Nup84p complex. J. Cell Biol. 149:41-5.

Siomi, H. and G. Dreyfuss. 1995. A nuclear localization domain in the hnRNP A1 protein. J. Cell. Biol. 129:551-60.

Snow, C.M., A. Senior and L. Gerace. 1987. Monoclonal antibodies identify a group of nuclearpore complex glycoproteins. J. Cell. Biol. 104:1143-56.

Stavru, F., B.B. Hulsmann, A. Spang, E. Hartmann, V.C. Cordes and D. Görlich. 2006. NDC1: a crucial membraneintegral nucleoporin of metazoan nuclear pore complexes. J. Cell Biol. 173:509-519.

Stoffler, D., B. Fahrenkrog, and U. Aebi. 1999. The nuclear pore complex: from molecular architecture to functional dynamics. Curr. Opin. Cell Biol.11:391-401.

Stüven, T., E. Hartmann and D. Görlich. 2003. Exportin 6: a novel nuclear export receptor that is specific for profilin.actin complexes. EMBO J. 22(21):5928-40.

Titov, A. and G. Blobel. 1999. The Karyopherin Kap122p/Pdr6p Imports Both Subunits of the Transcription Factor Iia into the Nucleus. J. Cell. Biol. 147(2):235-246.

Thomas, F and U. Kutay. 2003. Biogenesis and nuclear export of ribosomal subunits in higher eukaryotes depend on the CRM1 export pathway. J. Cell Sci. 116:2409-2419.

Trotman, L.C., N. Moosberger, M. Fornerod, R.P. Stidwill and U.F. Greber. 2001. Import of adenovirus DNA involves the nuclear pore complex receptor CAN/Nup214 and histone H1. Nat. Cell Biol. 3:1092-1100.

Trotta, R., E. Lund, L. Kahan, A.W. Johnson and J.E. Dahlberg. 2003. Coordinated nuclear export of 60S ribosomal subunits and NMD3 in vertebrates. EMBO J. 22(11): 2841-2851.

Wälde, S., K. Thakar, S. Hutten, C. Spillner, A. Nath, U. Rothbauer, S. Wiemann and R.H. Kehlenbach. 2012. The nucleoporin Nup358/RanBP2 promotes nuclear import in a cargo- and transport receptor-specific manner. Traffic. 13:218-233.

Waldmann, I., C. Spillner and R.H. Kehlenbach. 2012. The nucleoporin like protein NLP1/hCG1 promotes CRM1dependent nuclear protein export. J. Cell Science. 125:144-154.

Walther, T.C., H.S. Pickersgill, V.C. Cordes, M.W. Goldberg, T.D. Allen, I.W. Mattaj and M. Fornerod. 2002. The cytoplasmic filaments of the nuclear pore complex are dispensable for selective nuclear protein import. J. Cell. Biol. 158:63-77.

Waterhouse, P.M., M.B. Wang and T. Lough. 2001. Gene silencing as an adaptive defence against viruses. Nature. 411:834-42.

Weideman, C.A., R.C. Netter, L.R. Benjamin, J.J. McAllister, L.A. Schmiedekamp, R.A. Coleman and B.F. Pugh. 1997. Dynamic interplay of TFIIA, TBP and TATA DNA. J. Mol. Biol. 271:61-75. 
Weirich, C.S., J.P. Erzberger, J.M. Berger and K. Weis. 2004. The N-terminal domain of Nup159 forms a beta-propeller that functions in mRNA export by tethering the helicase Dbp5 to the nuclear pore. Mol. Cell 16:749-760.

Weis, K. 2002. Nucleocytoplasmic transport: cargo trafficking across the border. Curr. Opin. Cell Biol. 14:328-335.

Weis, K. 2007. The nuclear pore complex: oily spaghetti or gummy bear? Cell 130(3): 405-407.

Wen, W., J.L. Meinkoth, R.Y. Tsien and S.S. Taylor. 1995. Identification of a signal for rapid export of proteins from the nucleus. Cell. 82:463-473.

Wente, S.R. and P. Rout. 2010. The Nuclear Pore Complex and Nuclear Transport. Cold Spring Harb Perspect. Biol. 2:a000562.

Xie J., M. Collart, M. Lemaire, G. Stelzer and M. Meisterernst. 2000. A single point mutation in TFIIA suppresses NC2 requirement in vivo. EMBO J. 19(4):672-682.

Xu, L., Y. Kang, S. Cöl and J. Massaguè. 2002. Smad2 Nucelocytoplasmic Shuttling by Nuceloporins CAN/Nup214 and Nup153 Feeds TGF $\beta$ Signaling Complexes in the Cytoplasm and Nucleus. Mol. Cell. 10:271-282.

Xylourgidis, N., P. Roth, N. Sabri, V. Tsarouhas and C. Samakovlis. 2006. The nucleoporin Nup214 sequesters CRM1 at the nuclear rim and modulates NF\{kappa\}B activation in Drosophila. J. Cell Sci. 119: 4409 - 4419.

Yamada, J., J.L. Phillips, S. Patel, G. Goldfien, A. Calestagne-Morelli, H. Huang, R. Reza, J. Acheson, V.V. Krishnan, S. Newsam, A. Gopinathan, E.Y. Lau, M.E. Colvin, V.N. Uversky and M.F. Rexach. 2010. A bimodal distribution of two distinct categories of intrinsically-disordered structures with separate functions in FG-nucleoporins. Mol Cell Proteomics. M000035-MCP201.

Yedavalli, V.S.,C. Neuveut, Y.H. Chi,, L. Kleiman and K.T. Jeang. 2004. Requirement of DDX3 DEAD box RNA helicase for HIV-1 Rev-RRE export function. Cell. 119: 381-92.

Yokomori, K., M.P. Zeidler, J.L. Chen, C.P. Verrijzer, M. Mlodzik and R. Tjian. 1994. Drosophila TFIIA directs cooperative DNA binding with TBP and mediates transcriptional activation. Genes Dev. 8:2313-2323.

Zeiner, G.M., N.R. Sturm, and D.A. Campbell. 2003. Exportin 1 Mediates Nuclear Export of the Kinetoplastid Spliced Leader RNA. Eukaryot. Cell.2:222-230.

Zeitler, B. and K. Weis. 2004. The FG-repeat asymmetry of the nuclear pore complex is dispensable for bulk nucleocytoplasmic transport in vivo. J Cell Biol. 167:583-90.

Zeng, Y. and B.R. Cullen. 2004. Structural requirements for pre-microRNA binding and nuclear export by exportin 5 . Nucleic Acids Res. 32:4776-4785.

Zhang, M.J. and A.I. Dayton. 1998. Tolerance of diverse amino acid substitutions at conserved positions in the nuclear export signal (NES) of HIV-1 Rev. Biochem. Biophys. Res. Commun. 243:113-116. 


\section{$6 \quad$ Appendix}

\subsection{Alignments}

\section{CRM1}

NCBI accession numbers:
B. taurus
H. sapiens
C. elegans
$R$. norwegicus
$X$. laevis
M. musculus
D. melanogaster
NM_001192261.1
NM- 003400.3
NM 171484.4
NM_053490.1
$\mathrm{NM}^{-} 001090265.1$
$\mathrm{NM}^{-} 134014.3$
$\mathrm{NM}^{-} 164818.1$

H. sapiens

D. melanogaster

M. musculus

$R$. norwegicus

$X$. laevis

C. elegans

Consensus
H. sapiens
D. melanogaster
M. musculus
$R$. norwegicus
$X$. laevis
C. elegans
Consensus
H. sapiens
D. melanogaster
M. musculus
$R$. norwegicus
$X$. Iaevis
C. elegans
Consensus

1

(1) MPAIMTMLADHAARQLLDFSQKLDINLLDNVVNCLYHGEGAQQRMAQEVL

(1) ---MATMLTSDEAGKLLDFSQKLDINLLDKIVEVVYTAQGEQLRLAQS IL

(1) MPAIMTMLADHAARQLLDF SQKLDINLLDNVVNCLYHGEGAQQRMAQEVI

(1) MPAIMTMLADHAARQLLDFSQKLDINLLDNVVNCLYHGEGAQQRMAQEVL

(1) MPAIMTMLADHAARQLLDFSQKLDINLLDNVVNCLYHGEGAQQRMAQEVL

(1) -MAVSAMEVLSEAKRQFAQGDRIDVTLLDQVVE IMNRMSGKEQAEANQI I

(1) MPA IMTMLADHAARQLLDFSQKLDINLLDNVVNCLYHGEGAQQRMAQEVL 51

(51) THLKEHPDAWTRVDTILEFSQNMNTKYYGLQILENVIKTRWKILPRNQCE

(48) TTLKEHPEAWTRVDS ILEYSQNQRTKFYALQILEEVIKTRWKVLPRNQCE

(51) THLKEHPDAWTRVDTILEFSQNMNTKYYGLQILENVIKTRWKILPRNQCE

(51) THLKEHPDAWTRVDTILEFSQNMNTKYYGLQI LENVIKTRWKILPRNQCE

(51) THLKEHPDAWTRVDTILEFSQNMNTKYYGLQILENVIKTRWKILPRNQCD

( 50 ) MSLKEERDSWTKVDAILQYSQLNESKYFALQILETVIQHKWKSLPQVQRE

(51) THLKEHPDAWTRVDTILEFSQNMNTKYYGLQILENVIKTRWKILPRNQCE 101

(101) GIKKYVVGLIIKTSSDPTCVEKEKVYIGKLNMILVQILKQEWPKHWPTFI

(98) GIKKYVVSLIIKTSSDPIVMEQNKVYLNKLNMILVHILKREWPRNWETFI

(101) GIKKYVVGLI IKTSSDPTCVEKEKVY I GKLNMI LVQILKQEWPKHWPTFI

(101) GIKKYVVGLI IKTSSDPTCVEKEKVY I GKLNMILVQILKQEWPKHWPTFI

(101) GIKKYVVGLIIKTSSDATCVEKEKVYIGKLNMILVQILKQEWPKHWPTFI

(100) GIKSYIITKMFELSSDQSVMEQSQLLLHKLNLVLVQIVKQDWPKAWPTFI

(101) GIKKYVVGLIIKTSSDPTCVEKEKVYIGKLNMILVQILKQEWPKHWPTFI 
H. sapiens

D. melanogaster

M. musculus

$R$. norwegicus

$X$. laevis

C. elegans

Consensus

H. sapiens

D. melanogaster

M. musculus

$R$. norwegicus

$X$. laevis

C. elegans

Consensus

H. sapiens

D. melanogaster

M. musculus

$R$. norwegicus

$X$. laevis

C. elegans

Consensus

H. sapiens

D. melanogaster

M. musculus

$R$. norwegicus

$X$. laevis

C. elegans

Consensus

H. sapiens

D. melanogaster

M. musculus

$R$. norwegicus

$X$. laevis

C. elegans

Consensus

H. sapiens

D. melanogaster

M. musculus

$R$. norwegicus

$X$. laevis

C. elegans

Consensus

H. sapiens

D. melanogaster

M. musculus

$R$. norwegicus

$X$. laevis

C. elegans

Consensus
151

200

(151) SDIVGASRTSESLCQNNMVILKLLSEEVFDESSGQITQVKSKHLKDSMCN

(148) SDIVGASKTNESLCMNNMVILKNLSEEVFDFSQGQITQTKAKHLKDTMCS

(151) SDIVGASRTSESLCQNNMVILKLLSEEVFDFSSGQITQVKAKHLKDSMCN

(151) SDIVGASRTSESLCQNNMVILKLLSEEVFDFSSGQITQVKAKHLKDSMCN

(151) SDIVGASRTSESLCQNNMVILKLLSEEVFDFSTGQITQVKAKHLKDSMCN

(150) TDIVDSSKNNETVCINNMNILSLLSEEVFDFGSQNLTQAKEQHLKQQFCG

(151) SDIVGASRTSESLCQNNMVILKLLSEEVFDFSSGQITQVKAKHLKDSMCN 201

250

(201) EFSQIFQLCQFVMENSQNAPLVHATLETLLRFLNWIPLGYIFETKLISTI

(198) EFSQIFTLCSFVLENSMNAAL I HVTLETLLRFLNWIPLGYIFETQQIETL

(201) EFSQIFQLCQFVMENSQNAPLVHATLETLLRFLNWIPLGYIFETKLISTL

(201) EFSQIFQLCQFVMENSQNAPLVHATLETLLRFLNWIPLGYIFETKLISTL

(201) EFSQIFQLCQFVMENSQNAQLVHATLETLLRFLNWIPLGYIFETKLISTL

(200) QFQEVFTLCVS ILEKCPSNSMVQATLKTLQRFLTWIPVGYVFETNITELI

(201) EFSQIFQLCQFVMENSQNAPLVHATLETLLRFLNWI PLGYIFETKLISTL 251

300

(251) IYKFLNVPMFRNVSLKCLTEIAGVSVSQ----YEEQFVTLFTLTMMQLKQ

(248) IFKFLSVPMFRNVTLKCLSEIAGLTAAN----YDENFATLFKDTMVQLEQ

(251) IYKFLNVPMFRNVSLKCLTEIAGVSVSQ----YEEQFETLFTLTMMQLKQ

(251) IYKFLNVPMFRNVSLKCLTEIAGVSVSQ----YEEQFETLFTLTMMQLKQ

(251) VYKFLNVPMFRNVSLKCLTEIAGVSVSQ----YEEQFVTLFTLTMMQLKQ

(250) SENFLSLEVYRVIALQCLTEISQIQVETNDP SYDEKLVKMFCSTMRHISQ

(251) IYKFLNVPMFRNVSLKCLTEIAGVSVSQ YEEQFVTLFTLTMMQLKQ 301

350

(297) MLPLNTNIRLAYSNGKDDEQNF IQNLSLFLCTFLKEHDQLIEKRLN ----

(294) IVGQNMNMNHVFKHGSDTEQELVLNLAMFLCTFLKEHGKLVEDAK-----

(297) MLPLNTNIRLAYSNGKDDEQNF IQNLSLFLCTFLKEHGQLLEKRLN ----

(297) MLPLNTNIRLAYSNGKDDEQNF IQNLSLFLCTFLKEHGQLLEKRLN ----

(297) MLPLNTNIRLAYSNGKDDEQNFIQNLSLFLCTFLKEHGQLIEKRLN - -- -

(300) VLSLDLDLAAVYKDASDQDQKLISSLAQFLVAFIKEHVHLIEVTDEPLTE

(301) MLPLNTNIRLAYSNGKDDEQNFIQNLSLFLCTFLKEHGQLIEKRLN 351

400

(343) ----LRETLMEALHYMLLVSEVEETEIFKICLEYWNHLAAELYRESPFST

(339) ----YVDYLNQALMYLVMISEVEDVEVFKICLEYWNSLVEDLYN---S-E

(343) ----LREALMEALHYMLLVSEVEETEIFKICLEYWNHLAAELYRESPFST

(343) ----LREALMEALHYMLLVSEVEETEIFKICLEYWNHLAAELYRESPFST

(343) ----LRETLMEALHYMLLVSEVEETEIFKICLEYWNHLAAELYRESPFST

(350) AKILMRESHDYAIQLLLKITLIEEMEVFKVCLDCWCWLTAELYRICPFIQ

(351) LRETLMEALHYMLLVSEVEETEIFKICLEYWNHLAAELYRESPFST 401

450

(389) SASPLLSGSQHFDVPPRRQLYLPMLFKVRLLMVSRMAKPEEVLVVENDQG

(381) FFHPTLESTKRQQVYPRRRFYAPILSKVRFIMI SRMAKPEEVLVVENENG

(389) SASPLLSGSQHFDI PPRRQLYLTVLSKVRLLMVSRMAKPEEVLVVENDQG

(389) SASPLLSGSQHFDI PPRRQLYLTVLSKVRLLMVSRMAKPEEVLVVENDQG

(389) SASPLLSGSQHFDVPPRRQLYLPVLSKVRLLMVSRMAKPEEVLVVENDQG

( 400 ) PSTLYGMMSQVREHP-RRQLYREYLSQLRSTMISRMAKPEEVLIVENDQG

( 401 ) SASPLLSGSQHFDVPPRRQLYLPVLSKVRLLMVSRMAKPEEVLVVENDQG 451

500

(439) EVVREFMKDTDSINLYKNMRETLVYLTHLDYVDTERIMTEKLHNQVNGTE

(431) EVVREFMKDTNSINLYKNMRETLVFLTHLDSVDTDRIMTLKLLNQVNGSE

(439) EVVREFMKDTDSINLYKNMRETLVYLTHLDYVDTEI IMTKKLQNQVNGTE

(439) EVVREFMKDTDSINLYKNMRETLVYLTHLDYVDTEI IMTKKLQNQVNGTE

(439) EVVREFMKDTDSINLYKNMRETLVYLTHLDYADTERIMTEKLHNQVNGTE

( 449 ) EVVREMVKDTDSIALYRNMRETLVYLTHLDNKDTEVKMTEKLASQVNGGE

(451) EVVREFMKDTDS INLYKNMRETLVYLTHLDYVDTERIMTEKL NQVNGTE 
H. sapiens

D. melanogaster

M. musculus

$R$. norwegicus

$X$. laevis

C. elegans

Consensus

H. sapiens

D. melanogaster

M. musculus

$R$. norwegicus

$X$. laevis

C. elegans

Consensus

H. sapiens

D. melanogaster

M. musculus

$R$. norwegicus

$X$. laevis

C. elegans

Consensus

H. sapiens

D. melanogaster

M. musculus

$R$. norwegicus

$X$. laevis

C. elegans

Consensus

H. sapiens

D. melanogaster

M. musculus

$R$. norwegicus

$X$. Iaevis

C. elegans

Consensus

H. sapiens

D. melanogaster

M. musculus

$R$. norwegicus

$X$. laevis

C. elegans

Consensus

H. sapiens

D. melanogaster

M. musculus

$R$. norwegicus

$X$. laevis

C. elegans

Consensus
501

550

(489) WSWKNLNTLCWAIGS ISGAMHEEDEKRFLVTVIKDLLGLCEQKRGKDNKA

(481) FSWKNLNTLCWAIGS ISGAFCEEDEKRFLVTVIKDLLGLCEQKKGKDNKA

( 489 ) WSWKNLNTLCWAIGS I S GAMHEEDEKRFLVTVIKDLLGLCEQKRGKDNKA

(489) WSWKNLNTLCWAIGS I SGAMHEEDEKRELVTVIKDLLGLCEQKRGKDNKA

(489) WSWKNLNTLCWAIGS I SGAMHEEDEKRELVTVIKDLLGLCEQKRGKDNKA

(499) FSWKNLNRLCWAVGS I SGTMVEEDEKRFLVLVIRDLLGLCEQKRGKDNKA

(501) WSWKNLNTLCWAIGS I SGAMHEEDEKRFLVTVIKDLLGLCEQKRGKDNKA 551

600

(539) IIASNIMYIVGQYPRFLRAHWKFLKTVVNKLFEFMHETHDGVQDMACDTF

(531) I IASNIMYVVGQYPRFLRAHWKFLKTVVNKLFEFMHETHDGVQDMACDTE

(539) IIASNIMY IVGQYPRFLRAHWKFLKTVVNKLFEFMHETHDGVQDMACDTE

(539) IIASNIMY IVGQYPRFLRAHWKFLKTVVNKLFEFMHETHDGVQDMACDTE

(539) IIASNIMYIVGQYPRFLRAHWKFLKTVVNKLFEFMHETHDGVQDMACDTF

(549) VIASNIMYVVGQYPRFLRAHWKFLKTVINKLFEFMHETHEGVQDMACDTE

(551) IIASNIMYIVGQYPRFLRAHWKFLKTVVNKLFEFMHETHDGVQDMACDTF 601

650

(589) IKIAQKCRRHFVQVQVGEVMPF I DEI LNN INT I I CDLQPQQVHTFYEAVG

(581) IKIAIKCRRYFVTIQPNEACTFIDE I LTTMSS I ICDLQPQQVHTFYEAVG

(589) IKIAQKCRRHFVQVQVGEVMPFI DEI LNN INT I I CDLQPQQVHTFYEAVG

(589) IKIAQKCRRHFVQVQVGEVMPF I DE I LNN INT I ICDLQPQQVHTFYEAVG

(589) IKIAQKCRRHFVQVQVGEVMPF I DE I LNN INT I ICDLQPQQVHTFYEAVG

(599) IKISIKCKRHFVIVQPAENKPFVEEMLENLTGI ICDLSHAQVHVFYEAVG

(601) IKIAQKCRRHFVQVQVGEVMPFIDEILNNINTI ICDLQPQQVHTFYEAVG 651

700

(639) YMIGAQTDQTVQEHLIEKYMLLPNQVWDS I IQQATKNVDILKDPETVKQL

(631) YMISAQVDQVQQDVLIERYMQLPNQVWDDI ISRASKNVDFLKNMTAVKQL

(639) YMIGAQTDQTVQEHLIEKYMLLPNQVWDS I IQQATKNVDI LKDPETVKQL

(639) YMIGAQTDQTVQEHLIEKYMLLPNQVWDS I IQQATKNVDI LKDPETVKQL

(639) YMIGAQTDQTVQEHLIEKYMLLPNQVWDS I IQQATKNVDILKDPETVKQL

(649) HIISAQIDGNLQEDLIMKLMDIPNRTWNDI IAAASTNDSVLEEPEMVKSV

(651) YMIGAQTDQTVQEHLIEKYMLLPNQVWDSI IQQATKNVDI LKDPETVKQL 701

750

(689) GSILKTNVRACKAVGHPFVIQLGRIYLDMLNVYKCLSENISAAIQANGEM

(681) GSILKTNVAACKALGHAYVIQLGRIYLDMLNVYKITSENIIQAIEVNGVN

(689) GSILKTNVRACKAVGHPFVIQLGRIYLDMLNVYKCLSENISAAIQANGEM

(689) GSILKTNVRACKAVGHPFVIQLGRIYLDMLNVYKCLSENISAAIQANGEM

(689) GSILKTNVRACKAVGHPFVIQLGRIYLDMLNVYKCLSENISAAIQANGEM

(699) LNILKTNVAACKSIGSSFVTQLGNIYSDLLSLYKILSEKVSRAVTTAGEE

(701) GSILKTNVRACKAVGHPFVIQLGRIYLDMLNVYKCLSENISAAIQANGEM 751

800

(739) VTKQPLIRSMRTVKRETLKLISGWVSRSNDPQMVAENFVPPLLDAVLIDY

(731) VNNQPLIKTMHVVKKETLNLISEWVSRSNDNQLVMDNFIPPLLDA I LLDY

(739) VTKQPLIRSMRTVKRETLKLISGWVSRSNDPQMVAENFVPPLLDAVLIDY

(739) VTKQPLIRSMRTVKRETLKLISGWVSRSNDPQMVAENFVPPLLDAVLIDY

(739) VTKQPLIRSMRTVKRETLKLISGWVSRSSDPQMVAENFVPPLLDAVLIDY

(749) ALKNPLVKTMRAVKREILILLSTFISKNGDAKLILDS IVPPLFDAVLFDY

(751) VTKQPLIRSMRTVKRETLKLISGWVSRSNDPQMVAENFVPPLLDAVLIDY 801

850

( 789) QRN-VPAAREPEVLSTMAIIVNKLGGHITAEIPQIFDAVFECTLNMINKD

( 781 ) QRCKVPSAREPKVLSAMAI IVHKLRQHITNEVPKIFDAVFECTLDMINKN

(789) QRN-VPAAREPEVLSTMAI IVNKLGGHITAEIPQIFDAVFECTLNMINKD

(789) QRN-VPAAREPEVLSTMAI IVNKLGGHITAEIPQIFDAVFECTLNMINKD

(789) QRN-VPAAREPEVLSTMATIVNKLGVHITAEIPQIFDAVFECTLNMINKD

(799) QKN-VPQAREPKVLSLLSILVTQLGSLLCPQVPS ILSAVFQCS I DMINKD

(801) QRN VPAAREPEVLSTMAI IVNKLGGHITAEIPQIFDAVFECTLNMINKD 


\section{Appendix}

H. sapiens

D. melanogaster

M. musculus

$R$. norwegicus

$X$. laevis

C. elegans

Consensus

H. sapiens

D. melanogaster

M. musculus

$R$. norwegicus

$X$. laevis

C. elegans

Consensus

H. sapiens

D. melanogaster

M. musculus

$R$. norwegicus

$X$. laevis

C. elegans

Consensus

H. sapiens

D. melanogaster

M. musculus

$R$. norwegicus

$X$. laevis

C. elegans

Consensus

H. sapiens

D. melanogaster

M. musculus

$R$. norwegicus

$X$. laevis

C. elegans

Consensus
851

900

(838) FEEYPEHRTNFFLLLQAVNSHCFPAFLA IPPTQFKLVLDS I IWAFKHTMR

(831) FEDFPQHRLSFYELLQAVNAHCFKAFLNI PPAQFKLVFDSVVWAFKHTMR

(838) FEEYPEHRTNFFLLLQAVNSHCFPAFLA I PPAQFKLVLDS I IWAFKHTMR

(838) FEEYPEHRTNFFLLLQAVNSHCFPAFLA I PPAQFKLVLDS I IWAFKHTMR

(838) FEEYPEHRTHFFLLLQAVNSHCFPAFLAIPPAQFKLVLDS I IWAFKHTMR

(848) MEAFPEHRTNFFELVLSLVQECFPVFMEMPPEDLGTVIDAVVWAFQHTMR

(851) FEEYPEHRTNFFLLLQAVNSHCFPAFLAIP PAQFKLVLDS I IWAFKHTMR 901

950

( 888 ) NVADTGLQILFTLLQNV-AQEEAAAQSFYQTYFCDILQHIFSVVTDTS--

( 881 ) NVADMGLNILFKMLQNL-DQHPGAAQSFYQTYFTDILMQI FSVVTDTS--

( 888 ) NVADTGLQILFTLLQNV-AQEEAAAQSFYQTYFCDILQHIFSVVTDTS--

( 888 ) NVADTGLQILFTLLQNV-AQEEAAAQSFYQTYFCDILQHIFSVVTDTS--

( 888 ) NVADTGLQILYTLLQNV-AQEEAAAQSFYQTYFCDILQHTFSVVTDTS --

( 898 ) NVAEIGLDILKELLARVSEQDDKIAQPFYKRYYIDLLKHVLAVACDSSQV

(901) NVADTGLQILFTLLQNV AQEEAAAQSFYQTYFCDILQHIFSVVTDTS 951

1000

(935) HTAGLTMHAS I LAYMFNLVEEGKISTSLNPGN PVNNQ I FLQEYVANLLKS

(928) HTAGLPNHAI ILAYMFSLVENRKITVNLGPIP--DNMIFIQEYVASLLKS

(935) HTAGLTMHAS ILAYMFNLVEEGKISTPLNPGN PVNNQMF IQDYVANLLKS

(935) HTAGLTMHAS I LAYMFNLVEEGKISTPLNPGS PVSNQMF IQDYVANLLKS

(935) HTAGLTMHAS ILAYMFNLVEEGKINTPLNQAS PLNNQLF IQEYVANLLKS

(948) HVAGLTYYAEVLCALFRAPEFSIKVPLNDANPSQPNIDYIYEHIGGNFQA

(951) HTAGLTMHASILAYMFNLVEEGKISTPLNPG PVNNQIFIQEYVANLLKS 1001 1050

(985) AFPHLQDAQVKLFVTGLFSLNQDIPAFKEHLRDFLVQ IKEFAGEDTSDLF

(976) AFTHLSDNQVKVFVTGLFNLDENVQAFKEHLRDFLIQIREATGEDDSDLY

(985) AFPHLQDAQVKLFVTGLFSLNQDIPAFKEHLRDFLVQ IKEFAGEDTSDLF

(985) AFPHLQDAQVKLFVTGLFSLNQDIPAFKEHLRDFLVQ IKEFAGEDTSDLF

(985) AFPHLQDAQVKLFVTGLFSLNQDIAAFKEHLRDFLVQ IKEYAGEDTSDLF

(998) HFDNMNQDQIRIIIKGFFSFNTEISSMRNHLRDFLIQIKEHNGEDTSDLY

(1001) AFPHLQDAQVKLFVTGLFSLNQDI PAFKEHLRDFLVQIKEFAGEDTSDLF 1051 1088

(1035) LEEREIALRQADEEKHKRQMSVPGIFNPHEIPEEMCD-

(1026) LEEREAALAEEQSNKHQMQRNIPGMLNPHELPEDMQDE

(1035) LEERETALRQAQEEKHKLQMSVPGI LNPHE I PEEMCD-

(1035) LEERETALRQAQEEKHKLQMSVPGI LNPHE I PEEMCD-

(1035) LEERESSLRQAQEEKHKLQMSVPGI LNPHE I PEEMCD-

(1048) LEEREAEIQQAQ----QRKRDVPGI LKPDEVEDEDMR-

(1051) LEERETALRQAQEEKHKLQMSVPGILNPHEIPEEMCD 


\section{Nup214}

NCBI accession numbers:

$\begin{array}{ll}\text { B. taurus } & \text { NM_001205443.1 } \\ \text { H. sapiens } & \text { NM_005085.2 } \\ \text { C. elegans } & \text { NM_060167.5 } \\ \text { R. norwegicus } & \text { NM_001162031 } \\ X . \text { laevis } & \text { NM_001086197.1 } \\ \text { M. musculus } & \text { NM_172268.2 } \\ \text { D. melanogaster } & \text { NM_143782.2 }\end{array}$

1

50

B. taurus

H. sapiens

C. elegans

D. melanogaster

$X$. Iaevis

M. musculus

$R$. norwegicus

Consensus

B. taurus

H. sapiens

C. elegans

D. melanogaster

$X$. Iaevis

M. musculus

$R$. norwegicus

Consensus

B. taurus

H. sapiens

C. elegans

D. melanogaster

$X$. Iaevis

M. musculus

$R$. norwegicus

Consensus

B. taurus

H. sapiens

C. elegans

D. melanogaster

$X$. Iaevis

M. musculus

$R$. norwegicus

Consensus

(1) MGDEMDAVIPEREMKDFQFRALKKVRIFDSPEELPKER----SSLLSVSN

(1) MGDEMDAMI PEREMKDFQFRALKKVRIFDSPEELPKER----SSLLAVSN

(1) -MSNEDVAEDVSQVTDFHFHTCRKFRLFSSKSDGYSQNEINIRNRVATSS

(1) ---MAQNAPDCEDTQDFQFKLHHKIVAFKKCG-EPRSN----VNLLAVSS

(1) -MEDDTDLPPERETKDFQFRQLKKVRLFDYPADLPKQR----SNLLVISN

(1) MGDEMDAMI PEREMKDFQFRALKKVRIFDSPEELPKER----SSVLTISN

(1) MGDEMDAMI PEREMKDFQFRALKKVRIFDSPEELPKER----SSVLTVSN

(1) MGDEMDAMI PEREMKDFQFRALKKVRIFDSPEELPKER SSLLAVSN 51

100

(47) KYGLVFAGGASG-LHVFSTKNLLIQNKPGDDPNKIVDKV--QSLLVPMKF

( 47 ) KYGLVFAGGASG-LQIFPTKNLLIQNKPGDDPNKIVDKV--QGLLVPMKF

(50) QLGVTFVTVNSNQLSCFHTKSLLGYKITRENMNVEVTDLPIKTIRLHGVV

(43) SRGLLFAG------SPTQPELKVIIVKDLVNAKSTAQQP--QARLVPLPS

( 46 ) KYGLLFVGGFMG-LKVFHTKDILVTVKPKENANKTVVGP--QGIHVPMNS

(47) KYGMLFAGGTNG-LNVFPTKSLLIQNKPGDDPNKIVDTI-QGLNVPMKF

(47) KYGMLFAGGTNG-LNVFPTKSLLIQNKPGDDPNKIVDTI--QGLNVPMKF

(51) KYGLLFAGG G L VF TK LLIQNKPGDDPNKIVD I QGL VPMKF 101

150

(94) PIHHLALSCDNLTLSVCMMSSEYGSI IAFFDVRTFSNEAKQQKRPFAYHK

(94) PIHHLALSCDNLTLSACMMSSEYGSI IAFFDVRTFSNEAKQQKRPFAYHK

( 100 ) LINDMGVNSDGTVLGVLHTKNNDVSVDVFDIKKICTSSSIEPFKPLCTTR

(85) IPNYIACSSDGNLLAVNHTQNG----TSLLSIYAVLSFMTPDVRPVYNIR

(93) PIHHLALSSDNLTLSVCMTSAEQGSSVSFYDVRTLLNESKQNKMPFASCK

(94) PVHHLALSCDSLTLSACMMSSEYGSI IAFFDVRTFSNQAKPLKRPFTYHK

(94) PVHHLALSCDNLTLSACMMSSEYGSI IAFFDVRTFSNQAKPLKRPFTYHK

(101) PIHHLALSCDNLTLSVCMMSSEYGSIIAFFDVRTFSN AK KRPF YHK 151 200

(144) LLKDAGGLVIDMKWNPAVSSMVAVCLADGSIAVLQVTETVKVCATLPS--

(144) LLKDAGGMVI DMKWNPTVPSMVAVCLADGSIAVLQVTETVKVCATLPS--

(150) VGTEQINQGSCLEWNPAFPDTFAASSTDRS LVAKINVQSPANQKLVGIG

(131) LAAEDHVHGVQLLWNPVLPNSLAVVLSNGALAMYALKEGGNFEMHSLDK-

(143) LLRDPSSSVTDLQWNPTLPSMVAVCLSDGSISVLQVTDTVSVFANLPA--

(144) VSNDASGMVNDMKWNPTVPSMVAVCLADGSISVLQVTDVVKVCATLPP--

(144) LSNGASGMVNDMKWNPTVPSMAAVCLADGSISVLQVTDVVKVCATLPP--

(151) L DA GMV DMKWNPTVPSMVAVCLADGSIAVLQVTD VKVCATLP 201 250
B. taurus
H. sapiens
C. elegans
D. melanogaster
$X$. laevis
M. musculus
R. norwegicus
Consensus

(192) --TVGVTSVCWSPKGKQLAVGKQNGTVVQYLPTLQEKKVIPCPPFYEADH

(192) --TVAVTSVCWSPKGKQLAVGKQNGTVVQYLPTLQEKKVIPCPPFYESDH

(200) KFGAVTTAISWSPKGKQLTIGDSLGKIVQLKPELEVVRSQHGPEN--KPN

(180) --NQQVKCGCWS PKGKQIVLGFPGGTVKQFKPDLTLAKTLLCPPN-IHDA

(191) --TLGVTSVCWSPKGKQLAVGKQNGTVVQYLPSLQEKKVIPCPSFYDSDN

(192) --STGVTCVCWSPKGKQLAVGKQNGTVVQYLPTLQEKKVIPCPPFYESDH

(192) --SSGVTCVCWSPKGKQLAVGKQNGTVVQYLPTLQEKKVIPCPPFYEADH

(201) T GVTSVCWSPKGKQLAVGKQNGTVVQYLPTLQEKKVIPCPPFYESDH 


\section{Appendix}

B. taurus

H. sapiens

C. elegans

D. melanogaster

$X$. laevis

M. musculus

$R$. norwegicus

Consensus

\section{B. taurus}

H. sapiens

C. elegans

D. melanogaster

$X$. Iaevis

M. musculus

$R$. norwegicus

Consensus

B. taurus

H. sapiens

C. elegans

D. melanogaster

X. laevis

M. musculus

$R$. norwegicus

Consensus

\section{B. taurus}

H. sapiens

C. elegans

D. melanogaster

$X$. laevis

M. musculus

$R$. norwegicus

Consensus

B. taurus

H. sapiens

C. elegans

D. melanogaster

$X$. Iaevis

M. musculus

R. norwegicus

Consensus

B. taurus

H. sapiens

C. elegans

D. melanogaster

$X$. Iaevis

M. musculus

$R$. norwegicus

Consensus
251

300

(240) PVRVLDVLWIGTYEFTIVYAAADGTLETAPDVVMALLPKKEEKHPEVFMN

(240) PVRVLDVLWIGTYVFAIVYAAADGTLETSPDVVMALLPKKEEKHPEIFVN

(248) YGRITGLCWLATTEWLVSLENG--------TDQDAYLMRCKKDKPTEWIQ

(227) PFDTIAIQWLSTFQFAVIFLQHGEDCS PSLYILNAPKAG-----APSYIN

(239) PVKVLDVLWLSTYVFTVVYAAADGSLEASPQLVIVTLPKKEDKRAERFLN

(240) PVRVLDVLWIGTYVFTIVYAGADGTLETCPDVVMALLPKKEEKHPEIFVN

(240) PVRVLDVLWIGTYVFTIVYAGADGALETCPDVVMALLPKKEEKHPEIFVN

(251) PVRVLDVLWIGTYVFTIVYAAADGTLETSPDVVMALLPKKEEKHPEIFVN 301

350

(290) FMEPCYGSC---TERQHRYYLSYIEEWDLVLAASAASTEVS ILARQSS--

(290) FMEPCYGSC---TERQHHYYLSYIEEWDLVLAASAASTEVSILARQSD--

(290) FHELSYSSSKWPLPPQLFPATQLLVDWNVVIVGNSKTSEISTVGKRDD--

(272) YYDICYSMN---GPRNHQFVFSHVPQWNLLLVVSANGVEVGIMRSTEAGD

(289) FTETCYSIC---SERQHHFFLNYIEDWEILLAASAASVDVGVIARPPD--

(290) FMEPCYSSC---TERQHHYYLSYIEEWDLVLAASAASTEVS ILARQND--

(290) FMEPCYSSC---TERQHHYYLSYIEEWDLVLAASAASTEVS ILARQSD--

(301) FMEPCYSSC TERQHHYYLSYIEEWDLVLAASAASTEVSILARQ D 351

400

(335) QNSWESWLLEDSSRAELPVTDKSDDSLPMGVAIDYTNQVEITITEE--KT

(335) QINWESWLLEDSSRAELPVTDKSDDSLPMGVVVDYTNQVEITISDE--KT

(338) --WQTWVPVEGES-IYLPTTSSGKDTVPIGVAVDRSMTDEVLLNPDGSQR

(319) TPAWQQLTLLDEARIEMPLSEDKDETFPLGFAFDTSTTHQLTINE---KK

(334) QVGWEQWLLEDSSRAEMPMTENNDDTLPMGVALDYTCQLEVFISES--QI

(335) QTNWESWLLEDSSRAELPVTDKSDDSLPMGVAIDYTNEVEVTINEE--KT

(335) QTNWESWVLEDSSRAELPVTDKSDDSLPVGVAIDYTNEVEVAINEE--KT

(351) Q WESWLLEDSSRAELPVTDKSDDSLPMGVAIDYTN VEVTINEE KT 401

450

(383) LPPAPVLMLLSTDGVLCPFYMINQNPGVRSLLRTPERLSLEGERQPKSSG

(383) LPPAPVLMLLSTDGVLCPFYMINQNPGVKSLIKTPERLSLEGERQPKSPG

(385) HRPSPLVLCLTNDGILTAHHI ISTFAAH I PCQMSS-------------

(366) LQTMPMVHVLSSDGELLSENFLNVLPTAVSVCSPP--------------

(382) LPPVPVLLLLSTDGVLCPFHVVNLNQGVKPLTTSPEQLSLDGEREMKVVG

(383) LPPAPVLLLLSTDGVLCPFYMINQNPGVRSLIKTLELISTEGERQPKSSG

(383) LPPAPVLLLLSTDGVLCPFYMINQNPGVRSLIKTLELISAEGERQPKSSG

(401) LPPAPVLLLLSTDGVLCPFYMINQNPGVRSLIKTPE LS EGERQPKS G 451

500

(433) ------SIPTTPTSPQAPQKLDVPAAAAPASVLPSSQAAPTSTFSLP--

(433) ------STPTTPTS SQAPQKLDASAAAAPASLPPSSPAAPIATFSLLPA

(420)

( 401 ) ---P-----PVADTSGQFKPLNMLLASEEEEOPAWAAS--PSKAPAATPA

(432) GTAVSTPPAPLTSVSAPAPPASAAPRSAAPPPYPFGLSTASSGAPTPVLN

(433) ------SFPGTPSSPQAPQNLDAPATASSPLPPVS--AAPTSTEPMPSA

( 433 ) ------SFPSTPPSPQAPQNLDAPATASSPLPPVS--AAPTATFPLPSA

(451) S P TPTS QAPQ LDAPASAA $P$ S AAPTATF L A 501

( 474 ) ---SAVFSFGSSSLKSSGSVPGEAPLSSSGSDGAKAALVSGTSPFPFAPP

(476) GGAPTVFSFGSSSLKSSATVTGEPPSYSSGSDSSKAAPGPGPSTFSFVPP

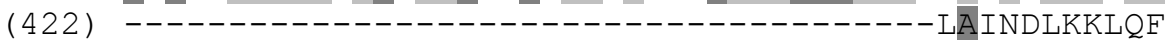

( 441 ) ASSDISFAFTPNTVTSTPAPSKDKQ--------------PSLFSGFGA

(482) PPASLAPAATPTKTTSQPAAAATSIFQPAGPAAGSLQP-PSLPAFSESSA

(474) AGSPSVFSFGPSSFKSSASVTGEPPLYPTGSDSSRAAPGSGTSTFSFAPP

(474) GGGPSVFSFGPSPFKS-SSIPGEPPPYPTGSDSSRAAPASGTPTFSFAPP

(501) A A VFSFGPSS KSS SV GE P SGSDSSKAAP G S FSFAPP 


\section{Appendix}

B. taurus

H. sapiens

C. elegans

D. melanogaster

X. laevis

M. musculus

$R$. norwegicus

Consensus

B. taurus

H. sapiens

C. elegans

D. melanogaster

$X$. laevis

M. musculus

R. norwegicus

Consensus

B. taurus

H. sapiens

C. elegans

D. melanogaster

$X$. Iaevis

M. musculus

$R$. norwegicus

Consensus

B. taurus

H. sapiens

C. elegans

D. melanogaster

$X$. laevis

M. musculus

$R$. norwegicus

Consensus

B. taurus

H. sapiens

C. elegans

D. melanogaster

$X$. laevis

M. musculus

$R$. norwegicus

Consensus

B. taurus

H. sapiens

C. elegans

D. melanogaster

$X$. laevis

M. musculus

$R$. norwegicus

Consensus
551

600

(521) SQASLAPTPVASPMMPSAASFSFGSSGFKPTLESTPMPSV--------SA

(526) SKASLAPTPAASPVAPSAASFSFGSSGFKPTLESTPVPSV--------SA

(433) DSQKPISAPPSDQTPVTKPSTVFG-----Q------------------

(475) AAAKAPAPQLSFGTAPTSSPVSFGAPTTNAAKPTTPFG-----------

(531) NNAANASAPSSFPFGAAMVSSNTAKVSAPPAMSFQPAMGTRPFSLATPVT

(524) SKGSLASTPAVAPVATSAAPFTFG---FKPTLESTPMS----------ST

(523) SKGTLAPTPAVAPVPTSAAPFTFGSSGFKPPLESTPMS----------SA

(551) S ASLASTPASAPVA SAASFSFGS FKP LESTPM S 601

650

(563) PNMGMKPTFPPSAPAVKVNLSEKFTAAATSAPVHNSQSTASMLPFS-ASK

(568) PNIAMKPSFPPSTSAVKVNLSEKFTAAATSTPVSSSQSAPPMSPFSSASK

( 458 ) -------KP-EAETLKSSLVGSPSSVQTPKPSS SLFNPKSIASNIETSQ

(513) G-FGTQATTTAMGSMFSASGANAFGGMALNKPAIASVTPRTAAPGSTVPA

(581) VQAATAPGFTPTPSTVKVNLKDKFNASDTPPPATISS--AAALSFTPTSK

(561) PNTGMKPSFPPSASSVKVNLNEKFTAVASSAPVHSSTSTPSVLPFSSSPK

(563) PNTGMKPSFPPSTSSVKVNLNEKFTAVASSAPVHSSTSIPSVLPFSSSPK

(601) PN GMKPSEPPSASSVKVNL EKFTAVATS PV SS S SMLPFSSSSK 651

700

(612) PASSGPLSHPAPLSASSSSLSSKSSATPSA-SGRAAQGSPSPVPSMVQKS

(618) PAASGPLSHPTPLSAPPSSVPLKSSVLPSP-SGRSAQGSSSPVPSMVQKS

(499) LTESKPSTPAAP-----------------------------SSQP---

(562) TPASAPAN----------------------------KP--LYTVP---

(629) PNATVPVKSQPTVI PSQASVQ-----PNRPFAVEAPQAPSSVSIASVQKT

(611) PTASGPLSHPTPLPASSSSMPLKSSVSPSPAAGRSTQTAPSSAPSTGQKS

(613) PPASGPLSHPTPLPASPSSMPLKPSVSPSPSVGRSAQTAPSSAPSTGQKS

(651) P ASGPLSHP PL AS SSM K S PSP AGRS Q APS PS QKS 701

750

(661) PRITPPVTKSGSPQPKSLQPPVTEKQGHQWKESDPVMAGIGEEIAHFQKE

(667) PRITPPAAKPGSPQAKSLQPAVAEKQGHQWKDSDPVMAGIGEEIAHFQKE

(515) --------KIASTPKS--------------------------E----A

(577) ----LTFTPVDTKPATSAPPQIADESLKPDDTEPI IKDMIALQIEAFSKD

(674) VRVNPPATKITPQPQR---SVALENQAKVTKESDSILNGIREEIAHFQKE

( 661 ) PRVNPPVPKSGSSQAKALQPPVTEKQRPQWKDSDPVLAGIGEEIAHFQKE

(663) PRVNPPVPKSGSSQARALQPPVTEKQRHQWKESDPVMAGIGEEIAHFQKE

(701) PRV PP K GS QAKSLQP V EKQ QWKESDPVMAGIGEEIAHFQKE 751

800

(711) LEELKARTSKACFQVGTSEEMKMLRTESDDLHAFLLEIKETTESLHGDIS

(717) LEELKARTSKACFQVGTSEEMKMLRTESDDLHTFLLEIKETTESLHGDIS

(525) IPKISDKTLEHKKAELIATKKQVLIERMDKINDSMAGAKDATMKLSFAVG

(623) IQKQKEQTKELLKGIAAPSALRAYAKRLDDLQELNEQAKDVEFELD--VQ

(721) LDDLKARTSRACFQVGSEEEKRQLRTESDGLHSFFLEIKETTESLRGEFS

(711) LEELKARTAKACLQVGTSEEMKMLRTESDDLHTFLFEIRETTESLHGDIS

(713) LEELKARTSKACFQVGTPEEMKMLRTESDDLHTFLFEIREATESLHGDIS

(751) LEELKARTSKACFQVGTSEEMKMLRTESDDLHTFL EIKETTESLHGDIS 801

850

(761) TLKTTLLEGFAGVEEAR-EQNERNRDSAYLHLLYKRPLDPRSEAQLQEIR

(767) SLKTTLLEGFAGVEEAR-EQNERNRDSGYLHLLYKRPLDPKSEAQLQEIR

(575) KVKTTIMECADVVRASLGDSKEVMDELKNLILSIERMSDRTQHTVKEMDF

(671) GLRQGLNEAYAIVAECR-GKLEIYRKPEITRLMNSSSCDPSGRRMLARLQ

(771) AMKIKNLEGFASIEDVQ-QRNKLKQDPKYLQLLYKKPLDPKSETQMQEIR

(761) TLKTTLLEGFAGVEEAR-EQHGRNHDSGYLHLLYKRPLDPKSEAQLQEIR

(763) TLKTTLLEGFAGVEEAR-EQHGRNHDSGYLHLLYKRPLDPKSEAQLQEIR

(801) TLKTTLLEGFAGVEEAR EQ ERN DSGYLHLLYKRPLDPKSEAQLQEIR 


\section{Appendix}

851

900

B. taurus

H. sapiens

C. elegans

D. melanogaster

$X$. laevis

M. musculus

$R$. norwegicus

Consensus

B. taurus

H. sapiens

C. elegans

D. melanogaster

$X$. laevis

M. musculus

R. norwegicus

Consensus

B. taurus

H. sapiens

C. elegans

D. melanogaster

$X$. Iaevis

M. musculus

$R$. norwegicus

Consensus

B. taurus

H. sapiens

C. elegans

D. melanogaster

$X$. Iaevis

M. musculus

$R$. norwegicus

Consensus

B. taurus

H. sapiens

C. elegans

D. melanogaster

$X$. laevis

M. musculus

$R$. norwegicus

Consensus

B. taurus

H. sapiens

C. elegans

D. melanogaster

$X$. laevis

M. musculus

$R$. norwegicus

Consensus
( 810 ) RLHQYVKFAVQDVNDVLDLEWDRHLEQKKK--QKRLLVPERETLFNTLAN

(816) RLHQYVKFAVQDVNDVLDLEWDQHLEQKKK--QRHLLVPERETLFNTLAN

(625) EIDEKMELVAGVEDGNQVLEKLRNMSETEKLMRFNKLETAADLLNGKYEE

( 720 ) SYVAANEAQLRLAQQHVDLQWEQFQDVVRRNSKSRMHMPCLEGIYQRLTR

(820) RLNQYVKNAVQDVNDVLDLEWDQYLEEKQK--KKGIIIPERETLENSLAN

(810) RLHQYVKFAVQDVNDVLDLEWDRHLEQKKR--QRRLIVPERETLFNTLAN

(812) RLHQYVKFAVQDVNDVLDLEWDRHLEQKKR--QRRLIVPERETLFNTLAN

(851) RLHQYVKFAVQDVNDVLDLEWDRHLEQKKK QRRLIVPERETLFNTLAN 901

950

(858) NREI INQQRKRLNHLVDSLQQLRLYNQTSQWSLPLDVPSQSTTHSFDSDI

( 864 ) NRE I INQQRKRLNHLVDSLQQLRLYKQTSLWSLSSAVPSQSSIHSFDSDL

(675) CSDLIKKLRMSLS

(770) LQNLTSNQR

(868) HQEI INQQRPKLEQLVENLQKLRLYNQISQWNVP-----DSSTKSFDVEI

( 858 ) NREI INQQRKRLNQLVDSLQQLRLYNHTAPWSLPSALSTQSNSHSFDSDI

(860) NRE I INQQRKRLNHLVDSLQQLRLYNQTSPWNRPSTLSTQSDTPSFDSDL

(901) NREIINQQRKRLN LVDSLQQLRLYNQTS W LP L SQS T SFDSDL 951

1000

(908) ESLRNALLKTTLEAHPKPLPKVPAKLSPVKQAQLRNFLAKRKTPPVRSTA

(914) ESLCNALLKTTIESHTKSLPKVPAKLSPMKQAQLRNFLAKRKTPPVRSTA

(688) --------------EKES---------------LRKQAILSPLRLSS

(779) --------------IVQN--------------NIKSKLKERGLLQAA

(913) ENMQKTLSQTAIDTQTKPQAKLPAKISPVKQSQLRNFLSKRKTPPVRSLA

(908) E----CLLKTTIESHTKPSPRVPGKLSPAKQAQLRNFLAKRKTPPVRSTA

(910) E----HLLKTTIESHTKPSPRVPGKLSPAKQAQLRNFLAKRKTPPVRSTA

(951) E LLKTTIESHTKP PKVPAKLSP KQAQLRNFLAKRKTPPVRSTA 1001 1050

(958) PASLSRSAFLSQRYYEDLDEASSTSSISQSLESEDTRTSCK--------

(964) PASLSRSAFLSQRYYEDLDEVSSTSSVSQSLESEDARTSCK---------

(706) NLNQLRS

(798) LLDQEKSRTRTNEAVDTLTDS ILTMSLSQVVDSNAAKLTRER--------

(963) PANLSRSAFLAPSEFEDLDDVSSTSSLSDMADNDNRNPPPKEIER-----

(954) PASLSRSAFLSQRYYEDLDEGSSASSVAQPLEGEDARPTCTSVAQPLEGE

(956) PASLSRSAFLSQRYYEDLDEGSSASSVAHPLEGEDARPTPT---------

(1001) PASLSRSAFLSQRYYEDLDE SSTSSVSQ LE EDAR T 1051

1100

( 999 ) ------DDDAVVQVPRHAPVVRTPS IQPSLLPQATPFAKSHLIHGSQ-G ( 1005 ) -------DDEAVVQAPRHAPVVRTPS IQPSLLPHAAPFAKS HLVHGSSPG

(713)

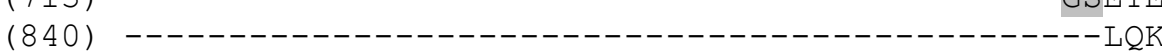

(1008) --QETPPPESTPVRVPKHAPVARTTSVQPGLGTASLPFQS-----GLHPA

( 1004 ) DAQP ICKEEEAVVPVPRHAPVVRTPS IQPSLLPQSMPFAKPHLIHSSSPA

(997) ----CREEEAVVPVPRHAPVVRTP SVQP SLLPQAMPFAKPHLIHSSSPA

(1051) DEEAVV VPRHAPVVRTPSIQPSLLP A PFAK HLIHGS PA 1101

1150

(1041) MMGTSMSTPASKI I PQGADSTMLATKTVKHGAPGPSHPISAPQAAAAAAL

(1048 VMGTSVATSASKIIPQGADSTMLATKTVKHGAPSPSHPISAPQAAAAAAL

(718) LALKVMRNVSKI IMDTREQIQRTELEFVRFQRDVKFQNFKKGKENLNFTQ

(843) IRNIVQLQKINVICPQRPDRVGLKSEVILETKRRAEQIKRAAAKPATANK

(1051 TSTPVAPSQSIRVIPQGADSTMLATKTVKHGAPN----ITAAQKAAVAAM

(1054) VMSSAVSTSATKVIPQGADSTMLATKTVKHGAPGPSHTVAAPQAAAAAAL

(1042) AMSSS ISTSATKVI PQGADTTMLATKTVKHGAPGPSHTVAAPQAAAAAAL

(1101) VM SSMST ASKIIPQGADSTMLATKTVKHGAP PSH IAAPQAAAAAAL 


\section{Appendix}

1151

1200
B. taurus
H. sapiens
C. elegans
D. melanogaster
$X$. laevis
M. musculus
$R$. norwegicus
Consensus

\section{B. taurus \\ H. sapiens \\ C. elegans \\ D. melanogaster \\ $X$. laevis \\ M. musculus \\ R. norwegicus \\ Consensus}

B. taurus

H. sapiens

C. elegans

D. melanogaster

$X$. Iaevis

M. musculus

$R$. norwegicus

Consensus

$B$. taurus
$H$. sapiens
$C$. elegans
$D$. melanogaster
$X$. laevis
$M$. musculus
$R$. norwegicus
Consensus

B. taurus

H. sapiens

C. elegans

D. melanogaster

$X$. laevis

M. musculus

$R$. norwegicus

Consensus
B. taurus
H. sapiens
C. elegans
D. melanogaster
$X$. laevis
M. musculus
$R$. norwegicus
Consensus

(1091) RRQMASQAPAVSTLTESTLKNVPQVVNVQELKNNAT--APSAAVGSSGPY (1098) RRQMASQAPAVNTLTESTLKNVPQVVNVQELKNNPA--TPSTAMGSSVPY

(768) PLEMSSLDGDAP-------------------------------------

(893) YTQAAVAPPSPP----DVAPT-------------------------P

(1097) RRQTASQIPAAS-LTESTLQTVPQVVNVKELKNNGPGPTIPTVIGPTVPQ

(1104) RRQMASQAPAMSTLTESTLKTVPQVVNVQELRSNPS--PPSAAMGSAVQH

(1092) RRQMASQAPAVSTLTESTLKNVPQVVNVQELKSNPS--PPSAAMGSSGPH

(1151) RRQMASQAPAVSTLTESTLK VPQVVNVQELK N PS AMGSS PH $1201 \quad 1250$

(1139) SAAKTPHPVLTPAPANQAKQGSLINALK-----PSGPPPASSQLSSGDKA

(1146) STAKTPHPVLTPVAANQAKQGSLINSLK-----PSGPTPASGQLSSGDKA

( 780 ) --QGK---SLTDAESIKVRQALVNQIQK--------R-------------

(911) AVAPMPQATVTVAPPLPKPMPSIPSVVE------KPG-------------

(1146) SAAQVIHQVLATVGSVSARQAAPAAPLKNPPASASS IAPQTWQGSAPNKP

(1152) SAAKTPHAVLTPVANSQAKQGSLINSFK-----PSGPTAASCQLSSGDKA

(1140) SAAKAPHAVLTPVAASQAKQGSLINSFK-----PSGPTPVSCQLSSGDKA

(1201) SAAK PH VLTPVAA QAKQGSLINSLK PSGP P S QLSSGDKA 1251

1300

(1184) S--VATKIETAVTSTPSTVG-QFSKPFSFS-SGTGFNFGIVTPTPSSNFT

(1191) S--GTAKIETAVTSTPSASG-QFSKPFSFSPSGTGFNFGIITPTPSSNFT

(804) -------------GIVKTRNVIVESYKKSENSAAMKNDLLDTSNLSNAI

(942) -------------VPTHPTTPVATPFSFSQSIPFVKTSTVTPTTNTVTP

(1196) AAQAIPKSDPSASQAPAPSVSQVNKPVSFSPAAGGFSFSNVTSAPVTSAL

(1197) VGQGTAKTESAATSTPSAAG-QLNKPFSFA-SPGTFTFGTITPTPSSSFT

(1185) VGPGTAKTESPVTSTPSTG--QLNKPFSFA-SPGTFTFGTITPAPSSSFT

(1251) G K ESA TSTPS QL KPFSFS S GGF FG ITPTPSS FT 1301 1350

(1230) -ATQGSAPPTKESSQLDAFSFGGGGKPFYETVPESLPSTGTMTSGSTGA

(1238) --AAQGATPSTKESSQPDAFSSGGGSKPSYEAIPESSPPS-----GITSA

(840) LKLSMTPRRVMPSSSLFSASPSTPSTKSDAATQADEPPIVKTVVVTVESP

(978) G-EAAKPGLSIFGGSTISSFSFGGGAAKSALSFGTGSPAVAAPTPKPNPL

(1246) GSSSAGCAATARDSNQASSYMFGGTGKSLGSEGSFSFASLKPAS---- - -

(1245) --ATPGAGPPTKEPTQLEAFSFGGGGKPFSEAIPGNSPATGATSAPSTSV

(1232) --ATPGAAPPTKESNQLEAFSFGGGGKPFCETIPGNPPATGPPSAPSTSV

(1301) AT GAAP TKESSQLDAFSFGGGGK ETIP PATG S TS 1351

1400

(1278) AGATPGESAPSSSRPAAPSGISLSTPAGKLEAPPSKLGELLFPSSLAGET

(1281) SNTTPGEPAASSSRPVAPSGTALSTTSSKLETPPSKLGELLFPSSLAGET

(890) AKPIASAPAVSSPLIKLNTTTATTTMTTPKVTVPKEEANKTQDQKPIIST

(1027) SAVEKPTPEPTKPK-----EQKAAESKEFKAVQPETE-ESKVPQKPKAET

(1290) SSSSSSVVEPTMSKPSVVTAASTTATVTSTTAASSKPGEGLFQGFSGGET

(1293) TAASLEDSAPSSSKPAAPPETTVSSASSKLETPPSKLGELLFPSSLAGET

(1280) TTTSLEDSAPTSSK-PATPETPVSTAS SKLETPPSKLGELLFPSSLAGET

(1351) S S D APSSSKP A S TALST SSKLETPPSKLGELLFPSSLAGET 1401

1450

(1328) LGSFSGLRVGQADEPTKPASKAPSPGVTSAGAQPTKTPAAPSGFSSPSPA

(1331) LGSFSGLRVGQADDSTKPTNKASS--TSLTSTQPTKTSGVPSGFNFTAPP

(940) PASSSIFSSGSLFGTKTQTPLVSK-

(1071) ENKSFGFGGFTGTGGTVGNTSSSP------------------FSFGGLG

(1340) LGSFSGLRVGQADEASKVEVAKTP----TA-AQPVKLPSNPVLFSFAGAP

(1343) LGSFSGLRVGQAEDSTKPVSKASS--TNLAGAQPAK----PSGVSFPNTS

(1329) LGSFSGLRVGQAEESTKPVSKASS--TNLAGAQPAK----PSGFSFPAAS

(1401) LGSFSGLRVGQADESTKP SKASS A AQP K PSGFSF A 


\section{Appendix}

1451

1500

B. taurus

H. sapiens

C. elegans

D. melanogaster

$X$. Iaevis

M. musculus

$R$. norwegicus

Consensus
B. taurus
H. sapiens
C. elegans
D. melanogaster
$X$. laevis
M. musculus
$R$. norwegicus
Consensus

\section{B. taurus}

H. sapiens

C. elegans

D. melanogaster

$X$. Iaevis

M. musculus

$R$. norwegicus

Consensus

\section{B. taurus \\ H. sapiens \\ C. elegans \\ D. melanogaster \\ $X$. Iaevis \\ M. musculus \\ $R$. norwegicus \\ Consensus}

\section{B. taurus}

H. sapiens

C. elegans

D. melanogaster

$X$. Iaevis

M. musculus

$R$. norwegicus

Consensus
B. taurus
H. sapiens
C. elegans
D. melanogaster
$X$. laevis
M. musculus
$R$. norwegicus
Consensus

(1378) ALGKPAEPPESTS----AAAPTAAASPSPSSSSTGVFGNLPLTSAGSSGA (1379) VLGKHTEPPVTSS----ATTTSVAPPAATSTSSTAVFGSLPVTSAGSSGV (964)

(1102)

(1373) VLGKPVEPSVTS--S-APAAPVSALSASTSSSSATVFGSVPLTSAAPSGL

(1451) LGK EP TSS SA S SSSS VFG VPLTSAG G $1501 \quad 1550$

(1424) ISFGGLFFSGSKTSFSFGGQ-PASSTAPSSTPP--STATPLPTSLPTLPF (1425) ISFGGTSLSAGKTSFSFGSQ-QTNSTVPPSAPPPTTAATPLPTSFPTLSF (964) ------EE--------------------STLTTGVPSLINSSLSISP

(1115) ----KSEP--------SSTATTSVATSASTAPFGIFSAALAKPSNSEPI

(1414) ----STAS----AFTQSGSKPAFTFGIPQSTSTTAGASSAIPASFQSLLV (1437) ISFGGAALASSKASFSFGNQQTSSSTASSATPTSTSVAPSLPASFPTLSF (1420) MSFSGASLASSKASFSFGNQQTSSAAAAAATPASTSVAPSLPASFPTLSF (1501) ISF G A A K SFSFG Q SSS ASTP TS A SLP SFPTLSF 1551 1600

(1471) GSPLSSGSAPTLPTGSGKSSEAAASSALPEKPGSNEASASTASLLGQQPP (1474) GSLLSSATTPSLPMSAGRSTEEATSSALPEKPGDSEVSASAASLLEEQQS (985) QEIEKASSKVETLNKTEEVKDEKSENEVTPDLKSEEPKSLETKVKEEP-(1152) TTVTSNTTTITSKPTNVIASSSVTDAPSVTTTNAVTSSTDPIGGLFSSVT (1456) SAAPATTTPSAPINSGLDVKQPIKPLSEPADSS SSQQTLTTQSAAEQVP (1487) GGLLSSPSASSLPVSSGKSTEEAAPPAVPDKSDSSEVSATTPSLPVQP-(1470) GGLLSSPSASSLPVSSGKITEEATPPAVPDKSDHSEVSASTPSLPLQPPS (1551) G LLSS S SLP SSGKSTEEAT AVPDKS SSE SAST SL 1601 1650

(1521) TPLPQAPLQTPDSGKKEPVLTQPAVSSPSTEAASTSVLVSTAEAAPAAPG (1524) AQLPQAPPQTSDSVKKEPVLAQPAVSNSGTAASSTSLVALSAEATPATTG (1033) ----KP---------------------------------AVQTPVK

(1202) ICKPNTPADTTKPANIFGGGLSAGSFSFGGTIDASKGLFGSTKPVATAPT (1506) TVTPAATATALPPPVPTIPSTAEAKIEGAAAPAIPASVISSQTVPFTST (1535) -QSTQASLQTSDPVKKEPVLVQTTDSSP-SRPASSASFVASTESMPVTLG (1520) AQLPQASLQTSDPVKKEPVPAQTTDS----RPSSSASLVASTESTPVAPG (1601) PQA QTSDP KKEPV Q S ASSA LV STEAVP G 1651

(1571) VPDVKTELPSPGPTVSVPGPATISAPGASSSIPVAPETSRPATTSSAVCS (1574) VPDARTEAVPPASSFSVPGQTAVTAAAISSAGPVAVETSSTPIASSTTSI (1042) ---------E-----------------------EETGSNIQKTPSFS (1252) SVTEANNKTDP----------IS------TTPSAISTTTATTTVSSPAV (1556) VLASQTPLAS-------------------TPAGGPTSQVPVLVTTAPP (1583) APDSKIEAVSPASTFAGPGQAAVATAVLPGAGSAATEASGTP-TTSTVSS (1566) GPDSKTEAVSPASTFAGPG-PAVVAAVLLGAGSAASEASGTPSTSSTVSS (1651) PDSKTE SPA T A PG V A PAA ETS TP TSST SS 1701 1750

(1621) AAPGPATETPVFGMATSDSSVFTQPSAASSSSAFSQLTSHTATAPSATPM

(1624) VAPGPSAEAAAFGTVTSGSSVFAQPPAASSSSAFNQLTNNTATAPSATPV (1057) FNSTTTPKSTSSTSSIFGGGLKTQTPSSSNSTNIFGARTTTTATPTPASN

(1285) VPAAVTAAVPATSSTTVTSSTAVPGSAFSFSNAFSTLSAGGAAAPTTSAS

(1585) VTTESAQTVSLTGQPVAGSSAFAQSTVTAASTPVFGQALASGAAPSPEAQ

(1632) SSPTSATETAVFGTATSGSSVFTQPPAASS SSAFSQLSSNTATAPSATPV

(1615) STPTPATETAVFGTATSGSSVFTQPPAASSSSAFNQLSSNTATAPSATPV

(1701) P A ETAVFGTATSGSSVFTQPPAASSSSAF QLSS TATAPSATPV 


\section{Appendix}

1751

1800

B. taurus

H. sapiens

C. elegans

D. melanogaster

$X$. laevis

M. musculus

$R$. norwegicus

Consensus

B. taurus

H. sapiens

C. elegans

D. melanogaster

$X$. laevis

M. musculus

$R$. norwegicus

Consensus

B. taurus

H. sapiens

C. elegans

D. melanogaster

$X$. Iaevis

M. musculus

$R$. norwegicus

Consensus

B. taurus

H. sapiens

C. elegans

D. melanogaster

$X$. Iaevis

M. musculus

$R$. norwegicus

Consensus

B. taurus

H. sapiens

C. elegans

D. melanogaster

$X$. laevis

M. musculus

$R$. norwegicus

Consensus

B. taurus

H. sapiens

C. elegans

D. melanogaster

$X$. laevis

M. musculus

$R$. norwegicus

Consensus
(1671) FGHVAASTAPSLFGQQTGSMASTAAAAPQVSSSGFGSPAFGTSTPGVFGQ

(1674) FGQVAASTAPSLFGQQTGSTASTAAATPQVSSSGFSSPAFGTTAPGVFGQ

(1107) TSSIFGG

(1335) APLAAKSP---------TATSTGNNSSNSVFGGGFAVATSTAAPVASPF

(1635) PTSSSVS-----------TSANSSTGFGTSAFGATG------GNGGFGQ

(1682) FGQVAAS----------- ITSTAAATPQASSSGFGSPAFGASAPGVFGQ

(1665) FGQVAAS-----------IASTAAATPQAS S SGFGSPAFGASAPGVFGQ

(1751) FG VAAS ASTAAATPQ SSSGF SPAFG SAPGVFGQ 1801 1850

(1721) PGFGQAPAFGQSTSSPASSFSFSQPGFSSVPAFGQSVSSTPTSTSGNVFG

(1724) TTFGQASVFGQSASSAASVFSFSQPGFSSVPAFGQPASSTPTSTSGSVFG

(1114) ---------GSKAASSPFGSFGQAGCQPAKTSNPATSTASVTFSFNTGA

(1375) QSAAKSPVSSANIFGS IPKAETSVFGGATTAPSNTTAAATPDAPPAGLFA

(1667) PSFGQAPLWKGPATS-QSTLPFSQPTFGTQPAFGQPAASTATSSAGSLFG

(1720) TAFGQTPAFGQATSSPASGFSFSQPGFSSVPAFGQSVSSTPASTSANVFG

(1703) TAFGQSPAFGQATSSPASGFSFSQPGFSSVPAFGQSVSSTPTSTSANVFG

(1801) AFGQAP FGQS SS AS FSFSQPGFSSVPAFGQS SSTPTSTSANVFG 1851

1900

(1771) ASSSTSSSSSFSFGQSS-TNNGGGLFGQNNPPAFGQSPGFGQ-GGSVFGG

(1774) AASSTSSSSSFSFGQSS-PNTGGGLFGQSNAPAFGQSPGFGQ-GGSVFGG

(1154) TSASAKPAGFGSFGAGASAKPSSVFGGSVTAPTVPNVDDGMEDDSMANGG

(1425) SAAISNPSPFGSPTTRAPASGGNIFGQAVKPSVFGQPAQAGDSGGSIFGG

(1716) CTSSASSFSFGQASNTSGTSTSGVLFGQSSAPVFGQSAAFPQ-AAPAFGS

(1770) ATSSTSSPGSFSFGQAS-TNTGGTLFGQNNPPAFGQSPGFGQ-GSSVFGG

(1753) ATSSTSSPGSFSFGQAS-TNTGGTLFGQNNPPAFGQSPGFGQ-GGSVFGS

(1851) ATSSTSSS SFSFGQAS TNTGG LFGQ NPPAFGQSPGFGQ GGSVFGG 1901

1950

(1819) TSAATTTASSSGFSFCQASGFGSSNTGSLFGQAASTSGTVFGQQSSSSSG

(1822) TSAATTTAATSGFSFCQASGFGSSNTGSVFGQAASTGGIVFGQQSSSSSG

(1204) G-1

(1475) GSASTPFGSS-------S-IFGGGNTQGAVGAPAAGSTSIFGQKVFGQSS

(1765) ASVSTTTTAS--FGFGQPAGFASGTSGSLFNPSQSGSTSVFGQQPASSSG

(1818) TSATTSTAAPSGFSFCQASGFGSSNTGSVFGQAANTGGSVFGQ-SSTSSG

(1801) TSATTSTAAPSGFSFCQASGFGSSNTGSVFGQAASSGGSVFGQ-SSTSSG

(1901) TSASTTTAASSGFSFCQASGFGSSNTGSVFGQAAST GSVFGQ SSSSSG 1951

2000

(1869) SVFGSGN-AgRGGGFFSGLGGKPSQDAANKNPFSSASG-GFGSTATPNTS

(1872) SVFGSGN-TGRGGGFFSGLGGKPSQDAANKNPFSSASG-GFGSTATSNTS

(1205) -----S------GGFMSGLGNARTSNTSGGNNPFAPKTSTGTSASSS--S

(1517) AAAPAAG-----GNIFSNPVGSPQ--ASPFGGGGNSIFGSPATAPPASGG

(1813) GLFGAGSGGASTVGLFSGLGAKPSQEAANKNPFGSPGSSGFGSAGASNSS

(1867) GVFGSGN-ATRGGGFFSGLGGKPSQDAANKNPFSSAGG-GFGSTAAPNTS

(1850) SVFGSGN-ATRGGGFFSGLGGKPSQDAANKNPFSSASG-GFGSTPTPNTS

(1951) SVFGSGN A RGgGFFSGLGGKPSQDAANKNPFSSA G GFGSTATSNTS 2001 2050

(1917) NLFGN-SGAKTFGGFASSSFGDQKPAGTFSSGGG-SVASQGFGFSTP---

(1920) NLFGN-SGAKTFGGFASSSFGEQKPTGTFSSGGG-SVASQGFGFSSP---

(1242) WLFGG--------GGNQQQQQQQKPSFSFNTAG--SSAQQASAPATG---

(1560) SIFGGGSSSGGFGSFTQTTPAQGGFGGGFGQGGGGSVAQTGFGSPQAPQQ

(1863) NLFGN-SGAKAFGFGG--TSFGDKPSATFSAGG--SVASQGFSFNSP---

(1915) NLFGN-SGAKTFGGFGSSSFGEQKPAGTFSSGGG-SVASQGFGFSTP---

(1898) NLFGN-SGAKTFGGFG-SSFGEQKPAGTFSSGGG-SVASQGFGFSTP---

(2001) NLFGN SGAKTFGGFG SSFGEQKPAGTFSSGGG SVASQGFGFSTP 
B. taurus

H. sapiens

C. elegans

D. melanogaster

$X$. laevis

M. musculus

$R$. norwegicus

Consensus

\section{B. taurus}

H. sapiens

C. elegans

D. melanogaster

$X$. laevis

M. musculus

$R$. norwegicus

Consensus

B. taurus

H. sapiens

C. elegans

D. melanogaster

$X$. Iaevis

M. musculus

$R$. norwegicus

Consensus
(1962) --NKTGGFGAAPVFGSPPTFGGSPGFGGVPAFGSAPAFTSPLGSTGGKVF

(1965) --NKTGGFGAAPVFGSPPTFGGSPGFGGVPAFGSAPAFTSPLGSTGGKVF

(1279) ---TSSVFGGAPKFGSQPAFGAKP-FGGGANAGLSKNASIFGGATSSTTN

(1610) QTTTPGGFGAKPVFGGSPAFGASPTFGGGATFGSPKGFGGFGGASPVASP

(1905) --TKTGGFGAAPVFGSPPTFGGSPGFGGSPAFGTAAAFSNTLGSTGGKVF

(1960) --NKTGGFGAAPVFGSPPTFGGSPGFGGVPAFGSAPAFTSPLGSTGGKVF

(1942) --NKTGGFGAAPVFGSPPTFGGSPGFGGVPAFGSAPAFTSPLGSTGGKVF

(2051) NKTGGFGAAPVFGSPPTFGGSPGFGGVPAFGSAPAFTSPLGSTGGKVF 2101 2150

(2010) GEGTAAASAGGFGFGSSSSTTSFGTLASQNAPTFGSLSQQSSGFGSQSGG

(2013) GEGTAAASAGGFGFGSSSNTTSFGTLASQNAPTFGSLSQQTSGFGTQSSG

(1325) NPATGGFAQFASGQKTSSLFGGGATPQTNTSIFGGGANTTPAPTSSVFGG

(1660) PPFGAAAKPAQGNIFETLGGQESGLSFGNLAQTGNSNAQKPAFGGSSEMN

(1953) GEGTSAATTGGFGFGSNSSTAAFGSLATQNTPTFGS I SQQSPGFGGQSSG

(2008) GEGTAAASAGGFGFGSSGNTASFGTLASQNAPTFGSLSQQTSGFGTPSSG

(1990) GEGTAAASAGGFGFGSS SNTASFGTLASQNAPTFGSLSQQTSGFGTPSSG

(2101) GEGTAAASAGGFGFGSSS TASFGTLASQNAPTFGSLSQQTSGFGS SSG $2151 \quad 2185$

(2060) FSGFGSS-------GGGFTFGSSNSSVQGFGGWRS

(2063) FSGFGSG-------TGGFSFGSNNSSVQGFGGWRS

(1375) GASANAN------------KP------TSFTSWR-

(1710) YR

(2003) FSGFGAGPGAAAGNTGGFGFGVSNPTSPGFGCWRS

(2058) FAGFGSG-------TGAFTFGSSNSSVQGFGGWRS

(2040) FSGFGSG-------TGAFTFGSSNSSVQGFGGWRS

(2151) FSGFGSG TGGFTFGSSNSSVQGFGGWRS

\subsection{Code for amino acids}

$\begin{array}{llllll}\text { A } & \text { Ala } & \text { alanine } & \text { Q } & \text { Gln } & \text { glutamine } \\ \text { C } & \text { Cys } & \text { cysteine } & \text { R } & \text { Arg } & \text { arginine } \\ \text { D } & \text { Asp } & \text { aspartate } & \text { S } & \text { Ser } & \text { serine } \\ \text { E } & \text { Glu } & \text { glutamate } & \text { T } & \text { Thr } & \text { threonine } \\ \text { F } & \text { Phe } & \text { phenylalanine } & \text { V } & \text { Val } & \text { valine } \\ \text { G } & \text { Gly } & \text { glycine } & \text { W } & \text { Trp } & \text { tryptophane } \\ \text { H } & \text { His } & \text { histidine } & \text { Y } & \text { Tyr } & \text { tyrosine } \\ \text { I } & \text { Iso } & \text { isoleucine } & & & \\ \text { K } & \text { Lys } & \text { lysine } & & & \\ \text { L } & \text { Leu } & \text { leucine } & & & \\ \text { M } & \text { Met } & \text { methionine } & & & \\ \text { N } & \text { Asn } & \text { asparagine } & & & \\ \text { P } & \text { Pro } & \text { proline } & & & \end{array}$




\subsection{Construction of CRM1-chimeras}

CRM1-chimeras were constructed according to the alignments and the secondary structure into the pEF-Myc vector (\#57).

Below, extracts of the CRM1 alignment (see 6.1) are shown for better comparison with the constructed chimeras. Used primer, restriction enzymes and amino acid residues are indicated. The amino acids in the orange box are partially modified due to the KpnI restriction site.

\section{$\underline{\text { Alignment Human/C. elegans CRM1 aa 539/549 - 588/598 }}$}

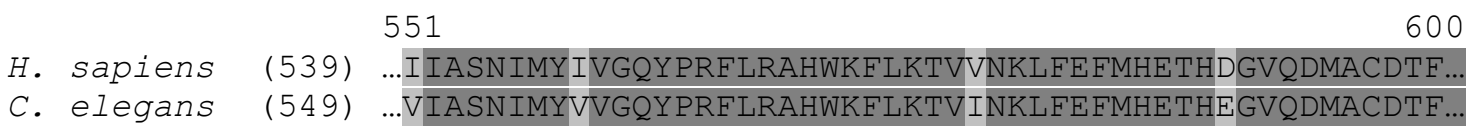

Chimeras HR 1-11/12-21
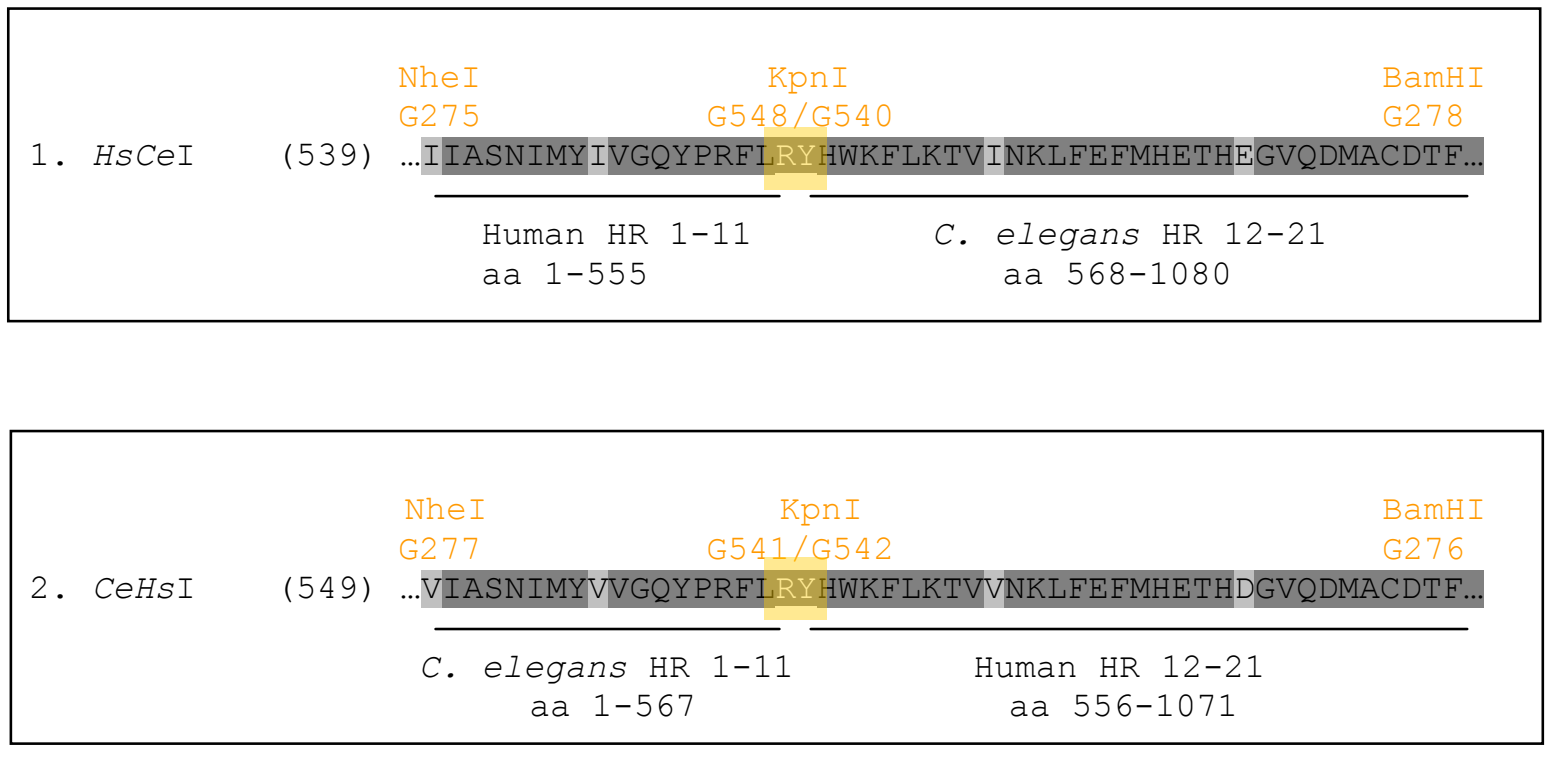


\section{$\underline{\text { Alignment Human/C. elegans CRM1 aa 351-450 }}$}
H. sapiens
351
400
C. elegans
(343)
...---
LRETLMEALHYMLLVSEVEETEIFKICLEYWNHLAAELYRESPFST
(350) ...AKILMRESHDYAIQLLLKITLIEEMEVFKVCLDCWCWLTAELYRICPFIQ
H. sapiens
401
450
C. elegans
(389) SASPLLSGSQHFDVPPRRQLYLPMLFKVRLLMVSRMAKPEEVLVVENDQG...
Consensus
(400) PSTLYGMMSQVREHP-RRQLYREYLSQLRSTMISRMAKPEEVLIVENDQG...

Chimeras HR 1-8/9-21
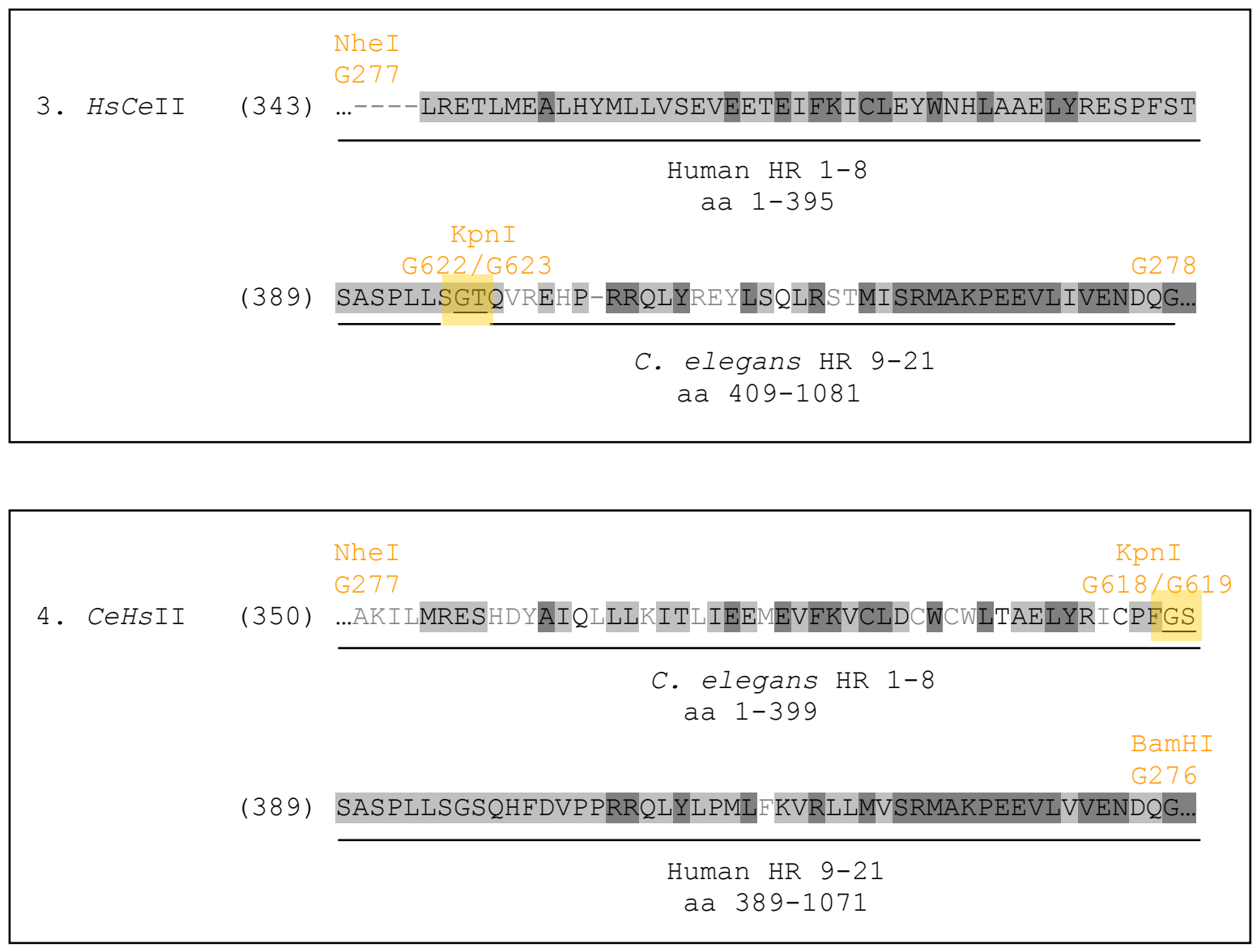


\subsection{Quantifications}

Fig. S1

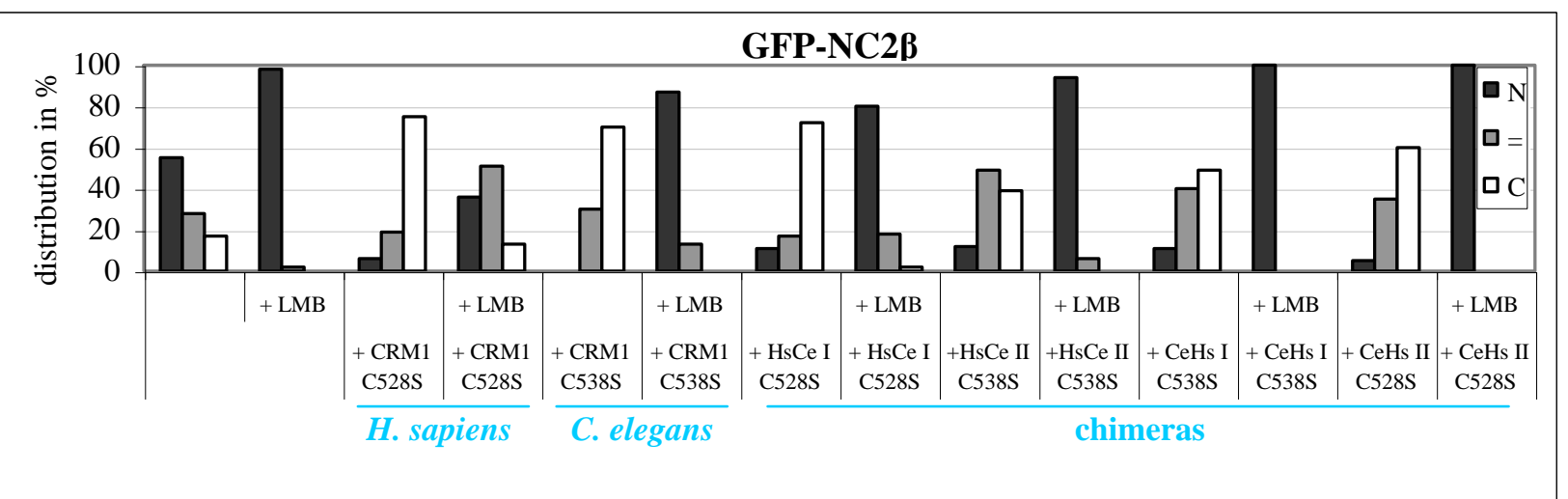

Distribution of GFP-NC2 $\beta$ cotransfected with CRM1-chimeras. HeLa cells were cotransfected with GFP-NC2 $\beta$ and CRM1 C528S/C538S mutants or with $H$. sapiens /C. elegans chimeras, respectively. After 40 hours, the cells were treated with or without $5 \mathrm{nM}$ LMB for three hours. After fixing, the cells were stained for HACRM1 using an anti-HA-antibody and analyzed by immunofluorescence microscopy. The localization of GFP$\mathrm{NC} 2 \beta$ was counted in at least 60 cells per sample ( $\mathrm{N}$ nuclear; = equally distributed; $\mathrm{C}$ cytoplasmic).

\section{Table S1}

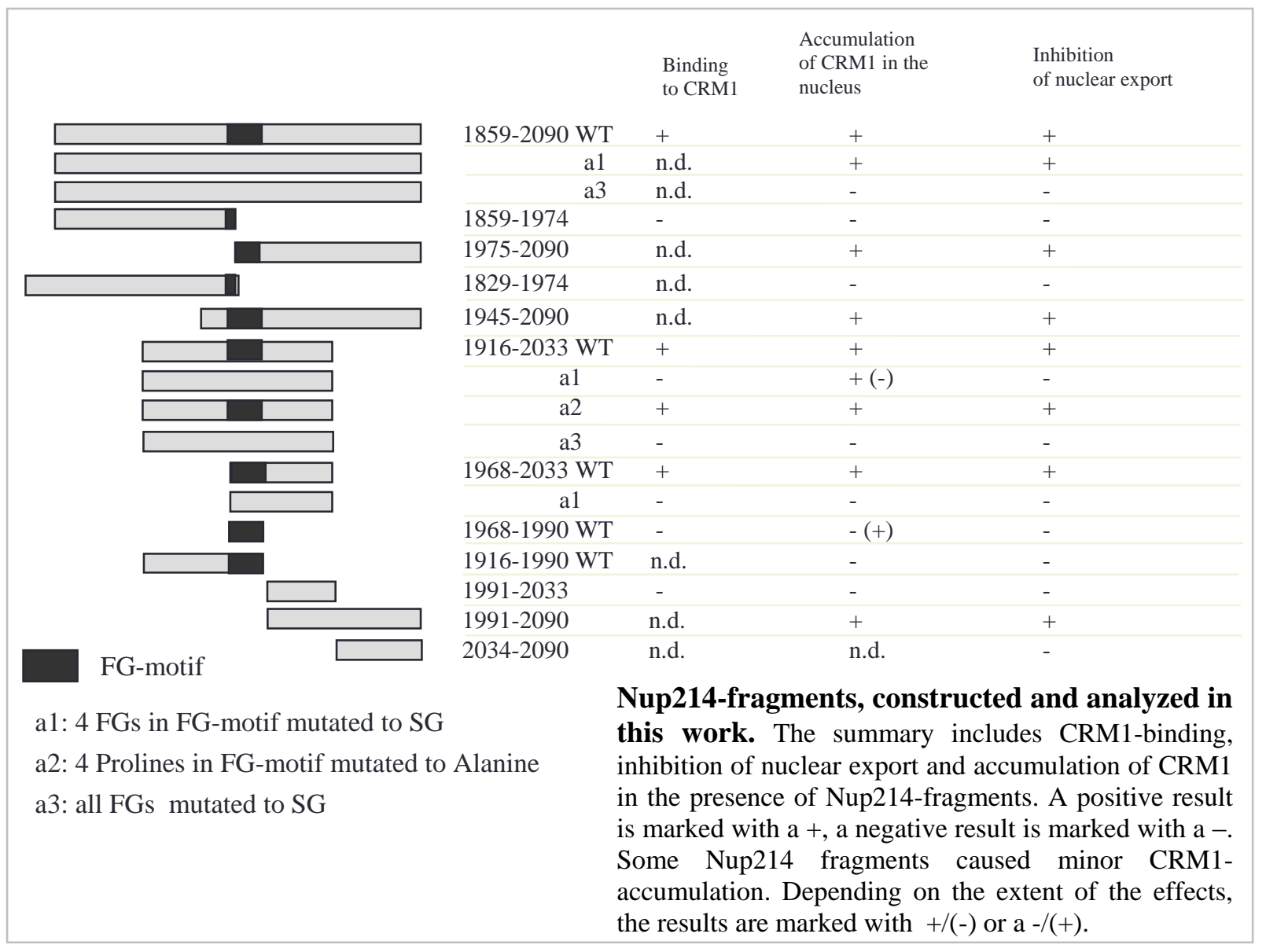


Fig S2

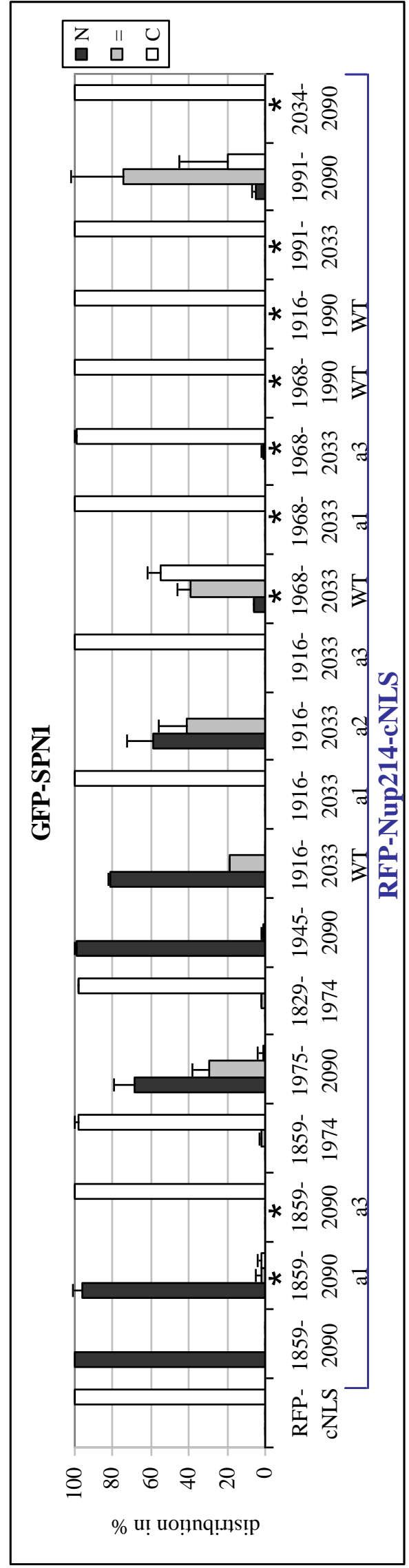

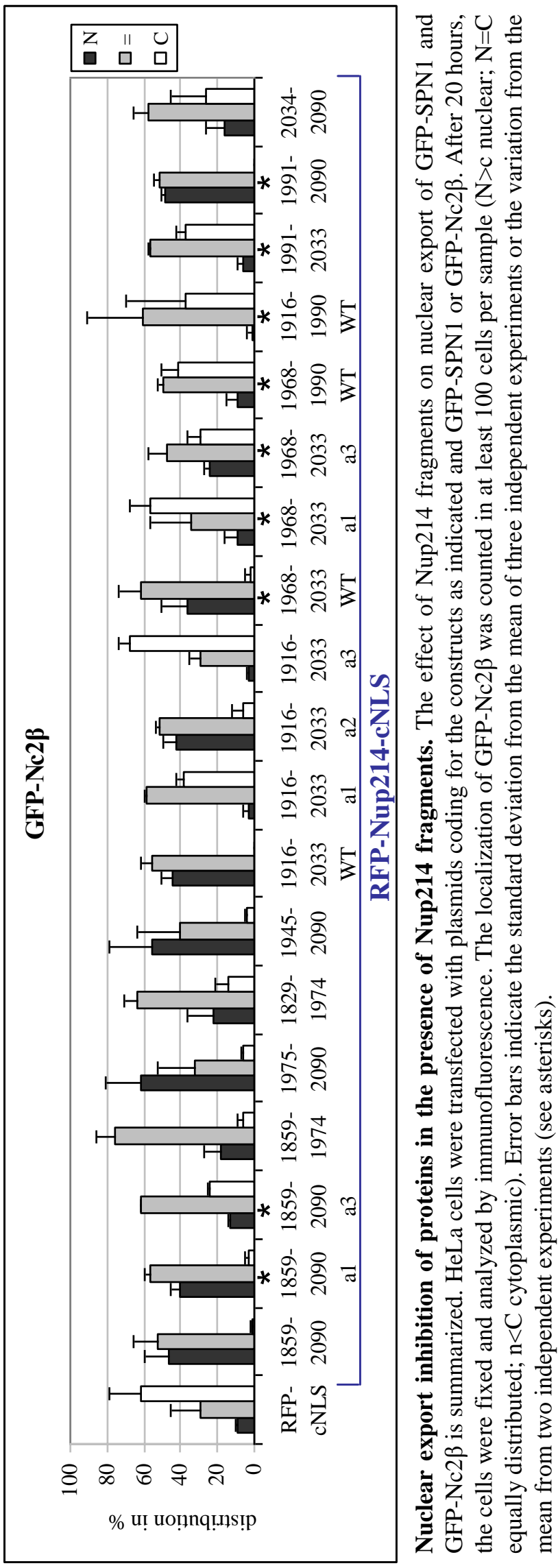




\subsection{Abbreviations}

Abbreviations

A

aa

ABL1

ATP

$\mathrm{bp}$

B. taurus

BSA

$\mathrm{C}$

$\mathrm{C}$

C. elegans/Ce

CRIME

CRM1

C-terminus

Da

D. melanogaster

DMEM

DMSO

DNA

dNTP

DTT

ECL

E. coli

EDTA

eIF2

$\mathrm{EtOH}$

FG-repeat

FCS

G

GAP

GDP

GEF

GFP

GSK-3

GST

GTP

GTPase adenine (DNA and RNA)

amino acid

Abelson murine leukemia viral oncogene homolog 1

adenosine-5-triphosphate

basepair

Bos taurus

bovine serum albumine

cytoplasmic

cytosine (DNA and RNA)

Caenorhabditis elegans

CRM1, IMportin $\beta$, Etcetera

chromosome region maintenance 1

carboxy terminus

dalton

\section{Drosophila melanogaster}

Dulbecco's modified eagles medium

dimethyl sulfoxide

desoxyribonucleic acid

2'-desoxynucleoside-5'-triphosphate

dithiothreitol

enhanced chemical luminescence

Escherichia coli

ethylenediaminetetraacetic acid

eukaryotic initiation factor 2

ethanol

phenylalanine glycine repeat

fetal calf serum

guanine (DNA and RNA)

GTPase-activating protein

guanosine-5'-diphosphate

guanine nucleotide exchange factor

green fluorescent protein

glycogen synthase kinase 3

glutathione-S-transferase

guanosine-5' -triphosphate

GTP-hydrolase 
Abbreviations

\begin{tabular}{|c|c|}
\hline GZMB & Göttinger Zentrum für Molekulare Biowissenschaften \\
\hline HEAT & Huntingtin Elongation Factor A Subunit TOR \\
\hline $\mathrm{HCl}$ & hydrochloric acid \\
\hline HEPES & [4-(2-hydroxyethyl)-1-piperazine]ethanesulfonic \\
\hline His & histidine tag \\
\hline HIV & human immunodeficiency virus \\
\hline HR & HEAT repeat motif \\
\hline H. sapiens /Hs & Homo sapiens \\
\hline IBB & importin $\beta$ binding \\
\hline IF & immunofluorescence \\
\hline INM & inner nuclear membrane \\
\hline IPTG & isopropyl- $\beta$-D-thiogalactopyranoside \\
\hline KLH & Keyhole Limpet Hemacyanin \\
\hline LB & Luria-Bertani \\
\hline LMB & Leptomycin B \\
\hline LTR & long terminal repeat \\
\hline MCS & multiple cloning site \\
\hline M. musculus & Mus musculus \\
\hline $\mathrm{N}$ & nuclear \\
\hline nct & nucleotide \\
\hline $\mathrm{NC} 2$ & negative cofactor \\
\hline NES & nuclear export signal \\
\hline NFAT & nuclear factor of activated T-cells \\
\hline cNLS & classical nuclear localization signal \\
\hline No & number \\
\hline NPC & nuclear pore complex \\
\hline N-terminus & amino terminus \\
\hline Nup & nucleoporin \\
\hline ONM & outer nuclear membrane \\
\hline PAGE & polyacrylamide gel electrophoresis \\
\hline PBS & phosphate buffered saline \\
\hline PCR & polymerase chain reaction \\
\hline $\mathrm{pH}$ & negative common logarithm of the proton \\
\hline PMSF & phenylmethylsulphonyl fluoride \\
\hline Ran & Ras-related nuclear protein \\
\hline $\mathrm{RCC} 1$ & Regulator of chromatin condensation 1 \\
\hline R. norwegicus & Rattus norwegicus \\
\hline RFP & red fluorescent protein \\
\hline
\end{tabular}


Abbreviations

$\begin{array}{ll}\text { Ran } & \text { Ras related nuclear protein } \\ \text { RanBP1 } & \text { Ran binding protein 1 } \\ \text { RCC1 } & \text { regulator of chromosome condensation 1 } \\ \text { RNA } & \text { ribonucleic acid } \\ \text { RNP } & \text { ribonucleoprotein } \\ \text { rpm } & \text { rotations per minute } \\ \text { SDS } & \text { sodium dodecyl sulphate } \\ \text { SV40 } & \text { Simian virus 40 } \\ \text { siRNA } & \text { small interfering RNA } \\ \text { SPN1 } & \text { Snurportin 1 } \\ \text { T } & \text { thymine (DNA and RNA) } \\ \text { TB } & \text { transport buffer } \\ \text { TAE } & \text { Tris /Acetate /EDTA } \\ \text { TBP } & \text { TATA binding protein } \\ \text { TE } & \text { Tris /EDTA } \\ \text { TFIIA } & \text { Transcription factor II A } \\ \text { Triton X-100 } & \text { 4-octylphenol polyethoxylate } \\ \text { Tween20 } & \text { polyoxyethylene (20) sorbitan monolaurate } \\ \text { U } & \text { uracile, in context of RNA } \\ \text { UV } & \text { ultraviolet } \\ \text { v/v } & \text { volume per volume } \\ \text { WGA } & \text { wheat germ agglutinin } \\ \text { WB } & \text { Western blot } \\ \text { WT } & \text { wild type } \\ \text { w/o } & \text { with or without } \\ \text { w/v } & \text { weight per volume } \\ \text { X. laevis } & \text { Xenopus laevis } \\ & \end{array}$


Physical units

$\begin{array}{ll}\mathrm{A} & \text { Ampere } \\ { }^{\circ} \mathrm{C} & \text { degree celsius } \\ \mathrm{g} & \text { gram } \\ \mathrm{xg} & \text { acceleration of gravity on earth } \\ \mathrm{hr} & \text { hour(s) } \\ \mathrm{l} & \text { liter } \\ \mathrm{M} & \text { molar (mol/l) } \\ \mathrm{min} & \text { minute(s) } \\ \mathrm{m} & \text { meter } \\ \mathrm{OD} & \text { optical density } \\ \mathrm{pH} & \text { potential hydrogen } \\ \mathrm{rpm} & \text { rotations per minute } \\ \mathrm{s} & \text { second(s) } \\ \mathrm{U} & \text { Unit } \\ \mathrm{V} & \text { volt }\end{array}$

\section{Prefixes}

$\mathrm{k}$

c

$\mathrm{m}$

$\mu$

n

$\mathrm{p}$ kilo- $\quad 10^{3}$

centi- $\quad 10^{-2}$

milli- $\quad 10^{-3}$

micro- $10^{-6}$

nano- $\quad 10^{-9}$

pico- $10^{-12}$ 


\section{Acknowledgements}

Nun, fast ganz zum Schluss dieser Arbeit (aber das soll nicht bedeuten, dass es nicht weniger wichtig ist) möchte ich einigen Menschen danken, die mich während meiner Arbeit fortwährend unterstützt haben.

Zuallererst einmal danke ich meinem Doktorvater Ralph Kehlenbach für die Bereitstellung dieses herausfordernden, wenn auch nicht immer ganz einfachen, Projektes. Bei Problemen oder Fragen hast Du immer Zeit gefunden und geholfen, dieses Projekt mit konstruktiven Lösungen in die richtige Richtung zu lenken. An dieser Stelle möchte ich mich auch bei meinen weiteren Thesis-Komitee Mitgliedern, Peter Rehling und Ralf Ficner bedanken, die großes Interesse an diesem Projekt gezeigt haben und es stets mit vielen Ideen bereichert haben.

Bedanken möchte ich mich auch bei meinen Laborkollegen Christiane Spillner, Annegreth Nath, Cornelia Frohnert, Ketan Thakar, Sarah Port, Inga Waldmann und auch bei der Arbeitsgruppe Schwappach für die entspannte Laboratmosphäre und super Unterstützung, wenn es um methodische oder projektbezogene Fragen ging. Christiane, die Arbeit mit Dir im letzten halben Jahr dieser Arbeit haben mir soviel Spaß gemacht, und ich bewundere Deinen unermüdlichen Einsatz, Deine Motivation, ohne Murren auch das 381. slide auszuzählen und immer noch guter Dinge zu sein Danken möchte ich auch Olaf Bernhard aus der AG B. Schmidt für die Massenspektrometrieanalysen.

Ein besonderer Dank gilt auch meinem Partner Volker Henschel, der mit seiner besonderen Art, mich zu motivieren, kritisieren und aufzubauen, immer unterstützt hat. Auch meiner Familie, die immer für mich da ist und mich liebevoll während dieser Arbeit unterstützt hat, möchte ich danken. 


\section{Curriculum vitae}

\section{Personal data}

Name: $\quad$ Stephanie Roloff

Date of birth: 23.07.1977 (Prenzlau)

Address: $\quad$ Mittelstr. 3g, 37077 Göttingen

\section{Education}

07.2008 - present

$09.2007-06.2008$

$10.2003-08.2007$

$04.2001-10.2003$

$08.2000-02.2001$

07.2000

$08.1997-06.2000$

06.1997

$1984-1997$
PhD thesis: „Analysis of CRM1- and Nup214- dependent nuclear export of proteins"

Department of Biochemistry I

Center for Biochemistry and Molecular Cell Biology

Georg-August University, Göttingen

Supervisor: Prof. Dr. Ralph Kehlenbach

Diploma thesis: "Die Rolle von Nup214 und seiner FG-repeats im CRM1-vermittelten Kernexport"

Department of Biochemistry I

Center for Biochemistry and Molecular Cell Biology

Georg-August University, Göttingen

Supervisor: Prof. Dr. Ralph Kehlenbach

Graduate and undergraduate studies: Biology

Georg-August-University, Göttingen

Sales clerk

dorana Berlin $\mathrm{GmbH}$, Berlin

Commercial clerk for purchasing and calculation

Ecotherm, Berlin

Sales clerk

Bär \& Ollenroth KG, Berlin

Apprenticeship: management assistant in wholesale and foreign trade

Bär \& Ollenroth KG, Groß Machnow

University entrance qualification

Gymnasium Templin

School education 
